\title{
THE PERFORMANCE OF ALTERNATIVE INTEREST RATE RISK MEASURES AND IMMUNIZATION STRATEGIES UNDER A HEATH-JARROW-MORTON FRAMEWORK
}

\author{
By \\ Şenay Ağca \\ Dissertation submitted to the faculty of \\ Virginia Polytechnic Institute and State University \\ in partial fulfillment of the degree of \\ Doctor of Philosophy \\ in \\ Finance
}

Approved By:

Don M. Chance, Chair

Randall Billingsley

Martin Day

Raman Kumar

Abon Mozumdar

March 25, 2002

Blacksburg, VA

Keywords: Term Structure Models, Arbitrage-free Pricing, Interest Rate Risk Measures, Immunization Strategies, Portfolio Formation Strategies 


\title{
THE PERFORMANCE OF ALTERNATIVE INTEREST RATE RISK MEASURES AND IMMUNIZATION STRATEGIES UNDER A HEATH-JARROW-MORTON FRAMEWORK
}

\author{
By Şenay Ağca \\ Committee: \\ Don M. Chance, Chairman \\ Randall Billingsley, Martin Day \\ Raman Kumar, Abon Mozumdar
}

\begin{abstract}
The Heath-Jarrow-Morton (HJM) model represents the latest in powerful arbitragefree technology for modeling the term structure and managing interest rate risk. Yet risk management strategies in the form of immunization portfolios using duration, convexity, and M-square are still widely used in bond portfolio management today. This study addresses the question of how traditional risk measures and immunization strategies perform when the term structure evolves in the HJM manner. Using Monte Carlo simulation, I analyze four HJM volatility structures, four initial term structure shapes, three holding periods, and two traditional immunization approaches (duration-matching and duration-and-convexitymatching). I also examine duration and convexity measures derived specifically for the HJM framework. In addition I look at whether portfolios should be constructed randomly, by minimizing their M-squares or using barbell or bullet structures. I assess immunization performance according to three criteria. One of these criteria corresponds to active portfolio management, and the other two correspond to passive portfolio management. Under active portfolio management, an asset portfolio is successfully immunized if its holding period return is greater than or equal to the holding period return of the liability portfolio. Under passive
\end{abstract}


portfolio management, the closer the returns of the asset portfolio to the returns of the liability portfolio, the better the immunization performance.

The results of the study suggest that, under the active portfolio management criterion, and with the duration matching strategy, HJM and traditional duration measures have similar immunization performance when forward rate volatilities are low. There is a substantial deterioration in the immunization performance of traditional risk measures when there is high volatility. This deterioration is not observed with HJM duration measures. These results could be due to two factors. Traditional risk measures could be poor risk measures, or the duration matching strategy is not the most appropriate immunization approach when there is high volatility because yield curve shifts would often be large.

Under the active portfolio management criterion and with the duration and convexity matching strategy, the immunization performance of traditional risk measures improves considerably at the high volatility segments of the yield curve. The improvement in the performance of the HJM risk measures is not as dramatic. The immunization performance of traditional duration and convexity measures, however, deteriorates at the low volatility segments of the yield curve. This deterioration is not observed when HJM risk measures are used. Overall, with the duration and convexity matching strategy, the immunization performance of portfolios matched with traditional risk measures is very close to that of portfolios matched with the HJM risk measures. This result suggests that the duration and convexity matching approach should be preferred to duration matching alone. Also the result shows that the underperformance of traditional risk measures under high volatility is not due to their being poor risk measures, but rather due to the reason that the duration matching strategy is not an appropriate immunization approach when there is high volatility in the market.

Under the passive portfolio management criteria, the performances of traditional and HJM measures are similar with the duration matching strategy. Less than $29 \%$ of the duration matched portfolios have returns within one basis point of the target yield, whereas almost all are within 100 basis points of the target yield. These results suggest that the duration matching strategy might not be sufficient to generate cash flows close to those of the target bond. The 
duration measure assumes a linear relation between the bond price and the yield change, and the nonlinearities that are not captured by the duration measure might be important.

When the duration and convexity matching strategy is used, more than $36 \%$ of the portfolios are within one basis point of the target with HJM risk measures. This dramatic improvement in the immunization performance of HJM measures is not guaranteed for traditional risk measures. In fact, there are certain cases in which the performance of traditional risk measures deteriorates with the duration and convexity matching strategy. In this respect, choosing the correct risk measure is more important than the immunization strategy when passive portfolio management is pursued.

Under active portfolio management criterion, there is no significant difference among bullet, barbell, minimum M-square, and random portfolios with both duration matching and duration and convexity matching strategies. Under the passive portfolio management criterion, bullet portfolios produce closer returns to the target for short holding periods when the duration matching strategy is used. With the duration and convexity matching strategy, bullet, barbell and minimum M-square portfolios produce closer returns to the target for short holding periods. Random portfolios perform as well as bullet, barbell and minimum M-square portfolios for medium to long holding periods. These results suggest that when the duration matching strategy is used, bullet portfolios are preferable to other portfolio formation strategies for short holding periods. When the duration and convexity matching strategy is used, no portfolio formation strategy is better than the other.

Under the active portfolio management criterion, minimum M-square portfolios are successfully immunized under each yield curve shape and volatility structure considered. Under the passive portfolio management criterion, minimum M-square portfolios perform better for short holding periods, and their performance deteriorates as the holding period increases, irrespective of the volatility level. This suggests that the performance of minimum M-square portfolios is more sensitive to the holding period rather than the volatility. Therefore, minimum M-square portfolios would be preferred in the markets when there are large changes in volatility. 
Overall, the results of the study suggest that, under the active portfolio management criterion and with the duration matching strategy, traditional duration measures underperform their HJM counterparts when forward rate volatilities are high. With the duration and convexity matching strategy, this underperformance is not as dramatic. Also no particular portfolio formation strategy is better than the other under the active portfolio management criterion. Under the passive portfolio management criterion, the duration matching strategy is not sufficient to generate cash flows closer to those of the target bond. The duration and convexity matching strategy, however, leads to substantial improvement in the immunization performance of the HJM risk measures. This improvement is not guaranteed for the traditional risk measures. Under the passive portfolio management criterion, bullet portfolios are preferred to other portfolio formation strategies for short holding periods. For medium to long holding periods, however, the portfolio formation strategy does not significantly affect immunization performance. Also, the immunization performance of minimum M-square portfolios is more sensitive to the holding period rather than the volatility. 


\section{DEDICATION}

\section{DEDICATE THIS DISSERTATION TO MY GRANDMOTHER ZELIHA INAK}

WHO PASSED AWAY IN 2001. 


\section{ACKNOWLEDGEMENTS}

I would like to thank my committee chair Don M Chance for all his guidance throughout the Ph.D. program and helping me prepare for the job market. He, as a committee chair, a coauthor, and a professor for whom I have worked as a graduate assistant for five years, was a big part of my life at Virginia Tech. I have learned a lot in working with him, and I will always admire his work ethics.

I would also like to thank Raman Kumar for his guidance during the Ph.D. program as Ph.D. director and also as my committee member. His comments on my dissertation as well as inputs from him regarding my job market preparation were extremely helpful. I also appreciate his suggestions and flexibility as Ph.D. director in helping me select the appropriate courses during the Ph.D. program.

I am grateful to Randy Billingsley, Abon Mozumdar, and Martin Day, for their insightful comments as my committee members. I really enjoyed working with them.

The utmost thanks and appreciations are for my mother Ülkü, my father Casim, my sister Tülay, and my brother Barış. They were there whenever I needed them. Without their support, none of this would be possible. I am lucky to have such great family members. My mother deserves a lot more than my thanks. She is the most wonderful person I have ever known. Whoever I am, and whatever I have accomplished, I owe it entirely to her. I am blessed to have such a marvelous mother and a role model to follow when (and if) I have my own children.

I also want to thank Saiyid Islam. Hopefully, we would be finishing the Ph.D. program together. We will always be in touch although he is trying to run away from me by going to the West Coast:-). Saiyid was a wonderful friend and colleague. Whenever I was not able to reach my family in Turkey, he was there for me. I cannot thank him enough for his friendship and support throughout the years. 
Also, my wonderful housemate Özlem Armutçuoğlu deserves my thanks. She helped me a lot, especially during the job market process, from selecting what to wear to soothing my nerves whenever I felt anxious. In her company, I always felt at home and yes, she, for me, is like a family member.

A special thanks goes out to Mahesh Pritamani. In the early stages of my Ph.D., his support helped me get out of several predicaments. Although he is very humble not to recognize it as anything important, his help and support was extremely valuable to me.

Among all my friends that I met throughout the years, I especially want to thank a few. I am thankful to Tunde for picking my interview calls in the office. Her help was very significant throughout my job search. Promod, Ali, Fatine, and Özge were wonderful assistants who reduced my teaching load with their valuable help. Andrew's encouragements during the job market were precious. Finally, I would like to thank Erik for the hours-long phone conversations that helped me think and talk about topics other than finance. 


\section{TABLE OF CONTENTS}

CHAPTER1

INTRODUCTION

CHAPTER 2

LITERATURE REVIEW 10

2.1. Traditional Risk Measures 10

2.2. Risk Measures Derived from Specific Term Structure Models 11

2.2.1. Interest Rate Risk Measures Derived from Equilibrium Term Structure Models 13

2.2.2. Heath, Jarrow and Morton Framework

2.2.3. Interest Rate Measures Derived from Specific HJM Models

2.3 Empirical Evidence on the Performance of Alternative Interest Rate Risk Measures 18

2.4. Portfolio Formation Strategies 19

2.4.1. Random Portfolios

2.4.2. Bullet and Barbell Portfolios 23

2.4.3. Minimum M-Square Portfolios 24 24 26

\section{CHAPTER 3}

TRADITIONAL RISK MEASURES 28

3.1. Duration 28

3.1.1. Summary of Duration Measures 30

3.2. Convexity 30

3.2.1. Summary of Convexity Measures 32

3.3 M-Square 32

\section{CHAPTER 4}

THE HEATH-JARROW-MORTON FRAMEWORK 35

CHAPTER 5

INTEREST RATE RISK MEASURES OF ONE-FACTOR

HEATH-JARROW-MORTON MODELS 39

5.1. Duration Measures of One-Factor HJM Models 39

5.1.1. Summary of Duration Measures for Certain One-Factor HJM Models $\left(D_{H J M}(t)\right)$ 41

5.2. Convexity Measures of One-Factor HJM Models 42

5.2.1. Summary of Convexity Measures for Certain One-Factor HJM Models (Conv ${ }_{H J M}(t)$ ) 43

\section{CHAPTER 6}

DATA AND METHODOLOGY 44

6.1. Forward Rate Data 44

6.2. Initial Yield Curves 45 
6.3. Forward Rate Volatilities____ 45

6.4. Coupon Bonds ____ 64

6.5. Holding Periods ___ 66

6.6. Immunization Criteria ___ 66

6.7. Portfolio Formation Strategies ___ 69

6.7.1. Barbell Portfolios —6.7. 70

6.7.2. Bullet Portfolios__ 70

6.7.3. Random Portfolios__ 71

6.7.4. Minimum M-Square Portfolios__ 74

6.8. Discretization of One-Factor HJM Models ___ 77

6.9. Random Variables ___ 78

6.10. Simulation _____________

CHAPTER 7

PERFORMANCE OF DURATION-MATCHED PORTFOLIOS

7.1. Constant Volatility One-Factor HJM Framework ___ 86

7.2. Exponential Decay Volatility One-Factor HJM Framework ___ 90

7.3. Constant Decay Volatility One-Factor HJM Framework___ 99

7.4. Humped Volatility One-Factor HJM Framework___ 110

7.5. Discussion on the Performances of Duration Matching Portfolios____ 117

CHAPTER 8

PERFORMANCE OF DURATION AND CONVEXITY MATCHED PORTFOLIOS___ 119

8.1. Constant Volatility One-Factor HJM Framework ___ 120

8.2. Exponential Volatility One-Factor HJM Framework ___ 127

8.3. Constant Decay Volatility One-Factor HJM Framework__ 138

8.4. Humped Volatility One-Factor HJM Framework___ 147

8.5. Discussion on the Performances of Duration and Convexity Matched Portfolios ___ 152

CHAPTER 9

PERFORMANCE OF MINIMUM M-SQUARE PORTFOLIOS____ 157

9.1. Constant Volatility One-Factor HJM Framework ___ 158

9.2. Exponential Decay Volatility One-Factor HJM Framework ___ 162

9.3. Constant Decay Volatility One-Factor HJM Framework___ 166

9.4. Humped Volatility One-Factor HJM Framework___ 171

9.5. Discussion of the Performance of Minimum M-Square Portfolios___ 175

\section{CHAPTER 10}

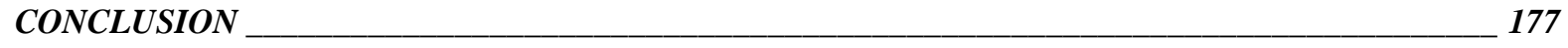

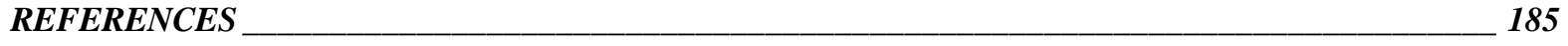

APPENDIX 1 __________ 194 
TECHNICAL APPENDIX 1 195

TECHNICAL APPENDIX 2

197

VITA

209 


\section{LIST OF TABLES}

Table 1

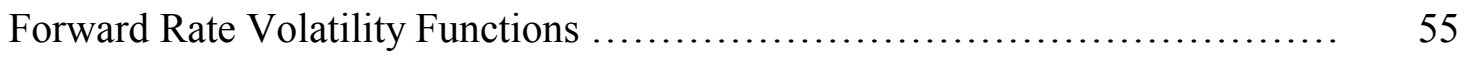

Table 2

Coupon Rates, Zero Coupon Bond Prices and Yields ..................... 67

Table 3

Bullet Portfolios for Duration Matching Strategy ......................... 72

Table 4

Bullet Portfolios for Duration and Convexity Matching Strategy .............. 73

Table 5

Minimum M-Square Portfolios for Duration Matching Strategy ............... 75

Table 6

Minimum M-Square Portfolios for Duration and Convexity Matching Strategy . 76

Table 7

Performance of Duration Matched Portfolios under Constant Volatility HJM

Framework ............................................................. 87

Table 8

Performance of Duration Matched Bullet and Barbell Portfolios under Constant

Volatility HJM Framework .......................................... 91

Table 9

Performance of Duration Matched Portfolios under Exponential Decay

Volatility HJM Framework

Table 10

Performance of Duration Matched Bullet and Barbell Portfolios under

Exponential Decay Volatility HJM Framework

Table 11

Performance of Duration Matched Portfolios under Constant Decay Volatility

HJM Framework

Table 12

Performance of Duration Matched Bullet and Barbell Portfolios under Constant

Decay Volatility HJM Framework .

108

Table 13

Performance of Duration Matched Portfolios under Humped Volatility HJM

Framework

Table 14

Performance of Duration Matched Bullet and Barbell Portfolios under Humped

Volatility HJM Framework 
Table 15

Performance of Duration and Convexity Matched Portfolios under Constant

Volatility HJM Framework

Table 16

Performance of Duration and Convexity Matched Bullet and Barbell Portfolios under Constant Volatility HJM Framework

Table 17

Performance of Duration and Convexity Matching Portfolios under Exponential Decay Volatility HJM Framework.

Table 18

Performance of Duration and Convexity Matched Bullet and Barbell Portfolios under Exponential Decay Volatility HJM Framework

Table 19

Performance of Duration and Convexity Matched Portfolios under Constant

Decay Volatility HJM Framework

Table 20

Performance of Duration and Convexity Matched Bullet and Barbell Portfolios under Constant Decay Volatility HJM Framework

Table 21

Performance of Duration and Convexity Matched Portfolios under Humped

Volatility HJM Framework

Table 22

Performance of Duration and Convexity Matched Bullet and Barbell Portfolios under Humped Volatility HJM Framework

Table 23

Performance of Minimum M-Square Portfolios under Constant Volatility HJM

Framework

Table 24

Performance of Minimum M-Square Portfolios under Exponential Decay

Volatility HJM Framework

Table 25

Performance of Minimum M-Square Portfolios under Constant Decay Volatility HJM Framework

Table 26

Performance of Minimum M-Square Portfolios under Humped Volatility HJM

Framework 


\section{LIST OF FIGURES}

Figure 1.

Initial Yield Curves and Forward Rate Curves ...................................... 46

Figure 2.

Forward Rate Volatility Curves ................................................ 51

Figure 3.

Forward Rate Volatility Functions ................................................... 60

Figure 4.

Histogram of Forward Rate Volatilities ......................................... 65

Figure 5.

Yield Curve Shifts....................................................................... 81 


\section{CHAPTER 1}

\section{INTRODUCTION}

Financial institutions intermediate between parties by exposing themselves to certain risks. One of the major risks is caused by the mismatch of the maturities of assets and liabilities. Banks usually generate funds with short-term liabilities, whereas their assets are by and large long-term. The liabilities of pension funds, on the other hand, are long term, and their assets may not match these long-term liabilities. Life insurance companies sell guaranteed investment contracts by which they are obliged to make certain interest payments at certain times. Therefore insurance firms want to make sure that they will have the assets to cover these liabilities.

When assets and liabilities do not mature at the same time, these financial institutions expose themselves to interest rate risk. When interest rates change, the response of different maturity assets and liabilities to this change will be different. These institutions want to guarantee that they will be able to cover their liabilities with their assets when interest rates change. The process of asset-liability management provides this protection by measuring and managing interest rate risk.

The earliest interest rate risk measure is developed by Macaulay (1938). He called this measure duration since it is a weighted average of the time to each cash payment. Hicks (1952) derived the same measure and showed that duration measures the change in the price of a bond when the yield changes. The duration measure assumes a linear relation between the bond price and the yield change. Therefore, it is an appropriate risk measure only when yield curve shifts are infinitesimal. Additionally, the duration measure of Macaulay (Macaulay's duration, henceforth) is restrictive, since it is theoretically valid only for flat yield curves and

parallel shifts in the yield curve. Fisher and Weil (1971) relaxed the assumption of a flat yield curve and develop another duration measure (Fisher-Weil duration, henceforth). Similar to Macaulay's duration, Fisher-Weil duration assumes parallel yield curve shifts. These two risk 
measures are generally referred to as traditional risk measures and are still widely used by asset-liability managers.

Since duration assumes a linear relation between the bond price and the yield change, it does not always perform well when yield curve shifts are non-infinitesimal. To capture the nonlinear relation between the bond price and the yield change, another measure is used in addition to the duration. This higher order measure is called convexity. Convexity measures the change in the basis risk when the yield changes. It is particularly important when yield curve shifts are large and, therefore, duration alone is a poor risk measure.

Although these traditional risk measures are still widely used, over the last decade, we have observed the introduction of more advanced risk measures. The rapid development in the fixed income markets literature, and availability and sophistication of technology, created an opportunity to develop these new measures. Since these risk measures depend on certain term structure models, term structure modeling constitutes one of the cornerstones of interest rate risk management today.

Term structure models can be categorized as equilibrium models and arbitrage-free models ${ }^{1}$. One of the most well known arbitrage-free models is Heath, Jarrow, and Morton (1992) (HJM, henceforth). In HJM models, the initial forward rate curve is exogenously given and the evolution of forward rates over time is determined by a specific continuous time stochastic process. HJM gives the conditions that have to be satisfied to ensure the existence of a unique equivalent martingale measure under which discounted bond prices are martingales. HJM is a popular model since it is very flexible in terms of the number of random factors that can be used in the model and different volatility structures that can be assumed for different maturity forward rates.

In one-factor HJM models, there is only one source of randomness. Hence, bond prices are perfectly correlated with each other. Nevertheless, one-factor models are still widely used, and most of the available models that are based on a single, short-term spot rate, called short rate models, can be represented as specific cases of one-factor HJM models. An additional attraction of one-factor models is that although they are relatively simple with respect to their

\footnotetext{
${ }^{1}$ Interested readers may refer to Ho (1995) and Yan (2001) for a discussion of equilibrium versus arbitrage-free interest rate models
} 
multi-factor counterparts, Litterman and Scheinkman (1991) and Chapman and Pearson (2001) show that $88 \%$ of the variation in the yield curve can be explained by only one factor.

Given that short rate models are still popular, we focus on the risk measures developed from specific one-factor HJM models. Since volatility functions are important determinants of HJM models, we select four widely used forward rate volatility functions. These are constant, exponential decay, constant decay, and humped volatility structures ${ }^{2}$. We determine the duration and convexity measures corresponding to these four specific HJM models ${ }^{3}$.

Our aim is to examine whether these more complex but more advanced risk measures provide any additional benefit over the simple traditional counterparts. If it is so, we would like to analyze under what conditions these benefits are observed. Although computational efficiency has increased through time, it is still costly for a firm to implement a more advanced risk measure that depends on a certain term structure model. The firm should have the necessary computer network that will make the updates in the term structure parameters available immediately at different branches at the same time, so that the same measure can be used for different activities. For example, a bank would like to use the same interest rate risk measure in the trading as well as banking book, and therefore the parameters that will be used in the risk measure should be available at the same time for both the banking and trading related staff. Another cost associated with the complex risk measures is that specialized personnel are needed to implement the term structure models. In these respects, the firm has to invest both in computer and human resources to use these complex risk measures. Therefore, it is important for a firm to know if it is beneficial to incur these costs and use complex risk measures instead of the simple traditional counterparts.

Empirical studies of Brennan and Schwartz (1983), Nelson and Schaefer (1983), Gultekin and Rogalski (1984), and Ho, Cadle and Theobald (2001) compare the performances of traditional risk measures with those of the complex risk measures derived from certain term

\footnotetext{
${ }^{2}$ By choosing specific volatility functions, we can exactly specify the duration and convexity measures corresponding to the HJM models with the selected volatility functions. We would not be able to specify these measures exactly if we were using the actual term structure of volatilities instead of specific volatility structures.

${ }^{3}$ These duration and convexity measures are either taken from the existing literature or derived if it was not available in the literature.
} 
structure models. All but Gultekin and Rogalski find that traditional risk measures perform as well as complex counterparts. Gultekin and Rogalski present some counterevidence.

Some of the reasons for this mixed evidence are due to different data sets, different portfolio formation strategies, and different analysis periods used by these studies as well as possible estimation and measurement errors. Yet another important reason is model risk, which is introduced as stochastic process risk in Bierwag (1987).

Model risk is the risk of selecting a term structure model that does not capture the evolution of interest rates. This can happen if the stochastic process conjectured to develop the duration measure is not the stochastic process of the real data. Any deviations from this conjecture will affect the results of the performance of alternative risk measures. Under different data sets and different analysis periods, stochastic process risk can lead to conflicting empirical evidence.

The portfolio formation strategies used in comparative and empirical studies are also different and these differences may also affect the immunization performances. The most common portfolio formation strategies considered are random, bullet and barbell portfolios. To form a random portfolio, securities are selected with a random drawing. A barbell portfolio consists of securities that have the lowest and highest durations. A bullet portfolio, on the other hand, has securities with the closest durations to the target holding period ${ }^{4}$. Fong and Vasicek (1983) propose another strategy. They develop a measure called M-square and show that if a duration-matched portfolio has the minimum M-square, it would have the best immunization performance.

When we examine the empirical studies that compare alternative risk measures, we observe that some studies form random portfolios and some use bullet or barbell portfolios. Another group of studies uses minimum M-square portfolios to control the stochastic process risk. In this respect, even if the data set and the analysis period are the same, empirical studies

\footnotetext{
${ }^{4}$ Holding period corresponds to the time period in which a target zero-coupon bond is held until the maturity. Therefore, holding period is the same as the duration of a target zero-coupon bond. In some studies such as Gultekin and Rogalski (1984), and Bierwag, Kaufman, Latta, and Roberts (1987), the term holding period is used as the rebalancing period. In this study, the duration of a target zero-coupon bond, which is its time to maturity, is equal to the holding period.
} 
can show different performances of traditional risk measures if different portfolio formation strategies are used.

To partially address these problems, we carry out simulations using real data. Initial forward rates and their volatilities as well as coupon rates of Treasury securities are inputs from real data. Using these inputs, forward rates are simulated under the selected HJM models and the risk measures corresponding to these models are used as benchmarks. For each scenario considered, one bullet, one barbell, one minimum M-square, and 100 random portfolios are formed. This approach allows us to analyze the effects of portfolio formation strategies on immunization performance. The immunization performance is examined according to three criteria. One of these criteria corresponds to active portfolio management and the other two correspond to passive portfolio management. Under active portfolio management, the aim of the asset-liability manager is to ensure that the return of the asset portfolio will be equal to or greater than the return on the liability portfolio. In this respect, we consider a portfolio successfully immunized under active portfolio management if its return is greater than or equal to that of the target bond. Under the passive portfolio management criterion, the aim of an asset-liability manager is to ensure that the return of the asset portfolio is as close as possible to the return on the liabilities. We consider two criteria that correspond to passive portfolio management. We analyze the number of portfolios that have returns within 100 basis points, and within one basis point of the target bond return.

Since we are generating interest rate changes using the selected HJM models, the study is strongly biased in favor of the HJM risk measures. In this respect, acceptable performance with traditional risk measures would suggest that firms are better off with traditional risk measures rather than the HJM counterparts. On the other hand, if in most of the cases, traditional risk measures underperform HJM risk measures, then the firms should prefer HJM risk measures to traditional ones.

Our results show that, according to the active portfolio management criterion, traditional duration measures perform as well as their HJM counterparts in $67 \%$ of the scenarios considered. These scenarios correspond to the cases where forward rate volatilities 
are relatively low ${ }^{5}$. This result suggests that when the yield curve shifts are small, duration is an acceptable risk measure. We also confirm the results of Litterman and Scheinkman (1991), and Chapman and Pearson (2001) in the sense that capturing the change in the level of the yield curve by traditional duration measures is sufficient in $67 \%$ of the cases.

When the forward rate volatilities are high, which is observed in the remaining $33 \%$ of the cases, the number of successfully immunized portfolios formed by HJM risk measures is at least $50 \%$ more than those formed by traditional risk measures. This suggests a significant underperformance of traditional risk measures. This underperformance can be due to two reasons. Traditional duration measures might be poor interest rate risk measures under certain yield curve shifts, or matching the portfolios only with the duration measure alone might not be sufficient when yield curve shifts are large.

When forward rate volatilities are high, we are more likely to observe more frequent large yield curve shifts. In these cases, we might consider higher order risk measures like convexity in addition to duration to capture the nonlinear relation between the bond price and the yield change. Our results show that when we form portfolios by matching with both duration and convexity, the performances of traditional risk measures improve substantially. We also observe improvement in the immunization performance of the HJM risk measures, but this improvement is not as great. On average, the difference in the number of successfully immunized portfolios formed by the HJM and traditional risk measures varies in a range of $10 \%$ to $20 \%$.

Our results suggest that there is a trade-off for the firm. If an asset-liability manager who follows an active portfolio management criterion forms the portfolios by matching with both duration and convexity, then there is a possible uncertain gain of $10 \%$ to $20 \%$ if the HJM risk measures are used. This gain is uncertain in the sense that, when HJM risk measures are used, there are possible estimation and calibration problems that might occur during the implementation of the HJM model. These problems might reduce the performance of these HJM risk measures. One should keep in mind that our results are strongly biased for the HJM

\footnotetext{
${ }^{5}$ We consider forward rate volatility as low if it is less than $2.25 \%$. To determine this volatility level, we analyze the forward rate volatility distribution and select the level that will make our results the most conservative. Any volatility level higher than $2.25 \%$ makes the results stronger in favor of the traditional interest rate risk measures.
} 
risk measures. In our study, we simulate the forward rates according to the selected HJM models, and therefore HJM risk measures are expected to perform well.

Under the passive portfolio management criteria, almost all duration-matched portfolios are within 100 basis points of the target yield. On the other hand, less than $29 \%$ of these portfolios are within one basis point of the target yield with both HJM and traditional risk measures. This suggests that the duration matching strategy alone is not sufficient to generate returns close to that of the target yield. This result may be due to assuming a linear relation between the bond price and the yield change by using only the duration measure. The nonlinearities that could not be captured by duration might be important for a portfolio to have cash flows closer to those of the target portfolio.

When the portfolios are matched with both the HJM duration and convexity measures, more than $36 \%$ of the portfolios have returns within one basis point of the target. This drastic improvement of HJM risk measures is not guaranteed for the traditional risk measures. There are cases where the performance of traditional risk measures deteriorates with the duration and convexity matching strategy. In this respect, under passive portfolio management, determining the correct risk measure is more important than the selection of an immunization strategy. An asset-liability manager will benefit significantly with the duration and convexity matching strategy if the correct interest rate risk measure is known. Otherwise, using the duration matching strategy might be a better alternative.

As previously noted, we also analyze the performance of alternative portfolio formation strategies. When we examine duration-matched portfolios, we find that bullet portfolios provide the closest returns to the target. For medium to long holding periods, the immunization performances of bullet, barbell and minimum M-square portfolios are similar to those of the random portfolios. These results suggest that for short holding periods, the most important determinant of the duration measure is time to maturity of the coupon bonds. Since bullet portfolios generally consist of bonds that have the closest times to maturity to that of the target, these portfolios have the closest returns to the target.

When we analyze portfolios that are matched with both duration and convexity, we do not observe significant differences among the performances of bullet, barbell, and minimum 
M-square portfolios. All produce very close returns to the target for short holding periods irrespective of the risk measure and the HJM model. For medium to long holding periods, the performances of these specific portfolio formation strategies are not significantly different than the performances of random portfolios. These results suggest that asset-liability managers might prefer bullet, barbell, or minimum M-square with the duration and convexity matching strategy for short holding periods.

Since M-square measures the time-to-payment variance of the bond price around the duration, minimizing the M-square of duration matched, or duration and convexity matched portfolios should reduce the variance around the duration. We expect that minimizing Msquare should improve the immunization performance if yield curve shifts are not captured by the duration and convexity measures. Our results show that the performance of minimum Msquare portfolios is very sensitive to the holding period but not sensitive to the volatility level. Even in the cases where volatility is inversely related to holding period, we observe that minimum M-square portfolios produce returns closer to the target yield for short holding periods. In these respects, asset-liability managers might prefer minimum M-square portfolios to other portfolio formation strategies when the differences in volatilities across the term structure are large. Also, all minimum M-square portfolios are successful irrespective of the risk measure used for duration and convexity. Nevertheless, minimum M-square portfolios formed by HJM risk measures have closer returns to the target than do the ones formed by traditional risk measures. Therefore, using the correct interest rate risk measure still has additional benefits, although these benefits are not very large.

The comparison of HJM risk measures with their simpler counterparts indicates the strengths and weaknesses of traditional risk measures. The study also provides a better understanding of the contribution of HJM models to immunization, and whether the additional complexity is worthwhile.

The dissertation is organized as follows. Chapter 2 surveys the literature on the traditional risk measures, the HJM framework, the HJM risk measures and portfolio formation strategies. Chapter 3 discusses traditional risk measures. Chapter 4 analyzes the HJM framework. Chapter 5 is on the HJM duration and convexity measures. Chapter 6 describes 
the data and the methodology. Chapter 7 and Chapter 8 examine the performances of duration-matched, and duration- and convexity-matched portfolios, respectively. Chapter 9 discusses the results for minimum M-square portfolios. Chapter 10 concludes the dissertation. 


\section{CHAPTER 2}

\section{LITERATURE REVIEW}

\subsection{Traditional Risk Measures}

The development of interest rate risk measures dates back to Macaulay (1938), Redington (1952), Hicks (1939), and Samuelson (1945). Macaulay introduced the concept of duration as a summary measure of the life of a bond. Duration is a weighted average of the time to each cash payment. The weight of each payment is its present value divided by the total present value of the cash payments. Independent of Macaulay, Hicks developed the same measure as the elasticity of capital value with respect to the discount factor and called it the average period. While analyzing the impact of interest rate changes on the value of financial institutions, Samuelson derived an average time period, which corresponds to Macaulay's duration. In the analysis of liability and asset allocation of life insurance firms, Redington developed the same measure and named it the mean term. In the same article, Redington introduced the term immunization as matching the mean term (Macaulay's duration) of assets and liabilities such that the portfolio will be immune to interest rate changes. In all of these early works, the duration measure assumes a constant yield as the discount factor. Under a flat yield curve and parallel shift of interest rates for all terms, Macaulay's duration provides an accurate immunization strategy.

Fisher and Weil (1971) relaxed the assumption of constant yield in Macaulay's duration and developed a new duration measure, henceforth Fisher-Weil duration. The discount factors of the cash flows in Fisher-Weil duration are derived from the current term structure. According to Fisher and Weil, a bond portfolio is immunized against interest rate changes if the holding period return of the portfolio is at least as large as the holding period return of the target bond ${ }^{6}$. Fisher-Weil duration does not assume a flat yield curve and provides an accurate hedging strategy for parallel shifts.

\footnotetext{
${ }^{6}$ Target bond is the zero coupon bond that matches the investment preferences of a certain investor. An example given in the Fisher and Weil study is a desire to have $\$ 1,000$ ten years hence by investing $\$ 558.39$ today. This
} 
Duration models are based on a linear relation between bond prices and interest rates. This assumption is valid for infinitesimal changes in interest rate. For non-infinitesimal changes the true non-linear relation between bond prices and interest rates should be taken into account to achieve an accurate immunization. In this respect, the second derivative of the bond price with respect to the interest rate provides the term, often called convexity, to be considered in addition to duration. Duration and convexity together provide a more accurate risk measure for non-infinitesimal changes in interest rates.

In addition to convexity, higher order duration measures are proposed to immunize a bond portfolio for non-parallel shifts as well as parallel shifts. One of these measures is Msquare, which is developed by Fong and Vasicek (1983) as a measure of immunization risk against arbitrary interest rate changes. M-square measures the weighted variance of the time to payments around the holding period, where the weights are the present values of the payments. Portfolios with low M-square will have low exposure to interest rate changes, and vice versa. Thus, it has been proposed to choose the portfolio with minimum M-square among the duration-matched portfolios.

Bierwag, Fooladi, and Roberts (1993) analyze M-square from the perspective of the two-factor model of Bierwag (1987) that uses two duration measures. The second order duration measure of Bierwag can be mapped onto M-square. Bierwag et al show that minimizing M-square corresponds to matching Bierwag's second order duration measure to the square of the holding horizon.

\subsection{Risk Measures Derived from Specific Term Structure Models}

After the Fisher and Weil study, the body of knowledge on interest rate risk measures accelerated mainly in two directions. One group of studies is devoted to the development of risk measures for different and more complex yield curve shift behavior such as multiplicative, or combined additive and multiplicative shifts. The other group focuses on specific term structure models and derives the risk measures implied by the assumed term structure model. Both of these groups consider single factor and multi factor duration 
measures. In this study, we focus on the risk measures derived from specific interest rate models $^{7}$.

As surveyed by Ho (1995) and Yan (2001), term structure models can be categorized as equilibrium models and arbitrage-free pricing models. Equilibrium models specify the market price of risk and estimate the parameters of the model from historical data on interest rates using current market fundamental or a combination of historical and fundamental analysis. These models use the estimated market price of risk to value bonds. Therefore, bond prices determined by equilibrium models may not match current bond prices. These models are useful for forecasting purposes as well as perceiving possible mispricing in bonds. They are, however, not practical for pricing purposes. To price bonds and other interest rate products, we prefer arbitrage-free pricing models.

Arbitrage-free pricing models assume that the underlying securities are correctly priced in the market and take the prices of the underlying securities as given. These models are calibrated to the observed prices. Since bond prices determined by arbitrage-free pricing models do not differ from the observed prices, these models are preferred for pricing purposes. One of the most widely referred arbitrage-free pricing model is Heath, Jarrow, and Morton (1992). Heath, Jarrow, and Morton develop a general arbitrage-free pricing framework that depends on the evolution of the instantaneous forward rate curve. HJM framework is appealing because of its flexibility to incorporate different volatility structures and different random factors.

In this study, we focus on a class of one-factor HJM models. For completeness, however, we review interest rate risk measures derived from both equilibrium and arbitragefree models. In this respect, the following subsections are organized as follows. First, we review the literature on the risk measures derived from equilibrium models. Then we survey existing literature on HJM models. The last subsection is devoted to the risk measures derived from HJM models.

\footnotetext{
${ }^{7}$ The reader interested in the literature related to the risk measures developed for certain yield curve behavior can refer to Bierwag, Kaufman, and Toevs (1981).
} 


\subsubsection{Interest Rate Risk Measures Derived from Equilibrium Term Structure Models}

Ingersoll, Skelton, and Weil (1978) introduce basis risk as the relative change in the bond price for an unexpected change in the interest rates, ceteris paribus. The spot interest rate is taken as a single stochastic factor deriving the term structure. The interest rate is assumed to follow the stochastic process:

$$
d r(t)=[\gamma+k(\alpha-r(t))] d t+\sigma d W(t)
$$

where

$$
d \gamma(t)=-\beta \gamma(t) d t+\eta \sigma d W(t)
$$

Here, $\alpha$ is the drift, $\mathrm{k}$ is the pulling factor, $\gamma$ is an extrapolative velocity, and $W$ is a standard Brownian motion or Wiener process. Under this stochastic, process Ingersoll et al derive the measure for the basis risk. The well-known Vasicek (1977) model is a special case of the above where $\gamma, \beta$, and $\eta$ equal zero. According to the developed measure, if basis risk increases linearly with maturity, Fisher-Weil duration is an accurate basis risk measure. If basis risk increases (decreases) with maturity at an increasing rate, Fisher-Weil duration is higher than the basis risk of short (long) maturity bonds.

Cox, Ingersoll, and Ross (CIR, henceforth) (1979) introduce the term stochastic duration to measure the relative basis risk of bonds. Stochastic duration is defined as the time to maturity of a zero coupon bond with the same basis risk as the coupon bond. Stochastic duration accommodates multiple interest rate shocks for both shape and location changes in the yield curve. The postulated stochastic process is the square root process of CIR (1985) where the spot rate is the only underlying stochastic factor is

$$
d r(t)=k(\alpha-r(t)) d t+\sigma \sqrt{r(t)} d W(t)
$$


CIR (1979) determine the equation for pricing zero coupon bonds consistent with the above stochastic process. Their stochastic duration measure is attained by taking the partial derivative of the bond price with respect to the spot rate and dividing it by the bond price. The stochastic duration measure depends on the parameters of the above stated interest rate process as well as the liquidity preferences of individuals.

Brennan and Schwartz (1983) develop a two-factor equilibrium model to determine the bond price. The two factors are the spot rate, $r$, and the yield on a consol bond, $l$. The stochastic processes of these two factors are jointly determined:

$$
\begin{aligned}
& d r(t)=\alpha_{1}(r(t), l(t), t) d t+\sigma_{1}(r(t), l(t), t) d W_{1}(t) \\
& d l(t)=\alpha_{2}(r(t), l(t), t) d t+\sigma_{2}(r(t), l(t), t) d W_{2}(t),
\end{aligned}
$$

where

$$
d W_{1}(t) d W_{2}(t)=\rho d t
$$

In this stochastic process, $\alpha_{1}$ and $\alpha_{2}$ are the drifts, and $\sigma_{1}$ and $\sigma_{2}$ are the volatilities of the spot rate and consol yield, respectively. Both drift and volatility are functions of time, spot rate, and consol yield. The derivation of the bond price according to above stochastic processes yields a partial differential equation with the boundary condition that the bond price at maturity is its face value. The appropriate hedging strategy is to construct the bond portfolio such that its estimated response the changes in the spot rate and consol yield will be the same as that of a discount bond with the appropriate maturity.

Nelson and Schaefer (1983) develop a $K$-factor model. The zero coupon bond price is a function of these $K$ factors, as well as time, and time to maturity. The factors are assumed to follow Gauss-Wiener processes. Each factor $k$ has drift of $\alpha_{k}$ that is a function of time and maturity, volatility of $\sigma_{k}$, and correlation $\rho_{j k}$ with factor $j$. The sensitivity of a bond's return to factor $k$ is considered to be a generalization of duration. To immunize a portfolio with the $K$ factor model, $K+1$ bonds are required. Immunization is achieved when the weighted sum of the sensitivities of each bond return with respect to factor $k$ is equal to the sensitivity of the target asset's return to factor $k$. This condition should be satisfied for all $K$ factors, and the 
weights in the summation should not change from one factor to the other. In this case, the more factors there are, the more will be the restrictions on the immunization strategy. This may limit the usefulness of the proposed strategy. Nelson and Schaefer analyze a two-factor strategy where the factors are the spot rate and the spread between the spot rate and the long rate. This two-factor strategy is based on the five-year rate, and the spread between the thirteen-year rate and the five-year rate. The stochastic process of the two-factor model of Nelson and Schaefer is as follows:

$$
\begin{aligned}
& d l(t)=\alpha_{1} d t+\sigma_{1} d W_{1}(t) \\
& d S(t)=k\left(\alpha_{2}-S(t)\right) d t+\sigma_{2} d W_{2}(t)
\end{aligned}
$$

where

$$
d W_{1}(t) d W_{2}(t)=0
$$

In this two-factor model, $\alpha_{1}$ and $\alpha_{2}$ are the drifts of a long rate and the spread between a long and a short rate, respectively. The volatilities of these two factors are given by $\sigma_{1}$ and $\sigma_{2}$. The spread is mean reverting, and the pull factor is $k$. Both innovations underlying the stochastic processes of the long rate and the spread are driven by Wiener processes. Note that the Wiener processes are uncorrelated.

\subsubsection{Heath, Jarrow and Morton Framework}

Heath, Jarrow, and Morton (1992) establish an arbitrage-free pricing framework based on the evolution of instantaneous forward rates over time. In HJM models, the initial forward rate curve is exogenously given, and there is a drift restriction that must be satisfied to guarantee the no arbitrage condition. HJM is a popular model since it is very flexible in terms of the number of random factors that can be used in the model and different volatility structures that can be assumed for different maturity forward rates ${ }^{8}$.

\footnotetext{
${ }^{8}$ The HJM framework and one-factor HJM models are analyzed in detail in Chapter 4. Therefore, we do not present any mathematical details related to the HJM models in this section
} 
In one-factor HJM models, there is only one source of randomness, a simple Brownian motion that drives all forward rates. Therefore, yields, bond prices, and changes in forward rates are perfectly correlated in one-factor models. Nevertheless, one-factor HJM models are flexible in the sense that all short rate models can be represented as special cases of one-factor HJM models. Baxter and Rennie (1998) ${ }^{9}$ show Ho and Lee (1986), Vasicek (1977), Hull and White (1993), Cox, Ingersoll, and Ross (1985), and Black and Karasinski (1991) models as special cases of one-factor HJM models. Multi-factor HJM models involve multiple Brownian motions, which allows different bonds to be affected by shocks in different ways. Recent developments related to HJM models are random field models of Kennedy (1994), Kennedy (1997), and Goldstein (2000), and the stochastic string model of Santa-Clara and Sornette (2001). In these models, instantaneous forward rates are imperfectly correlated with the forward rates of different maturities and each forward rate has its own shock. Another extension is the jump diffusion HJM models. Björk et al (1997) discuss the HJM methodology for jump diffusions and derive the conditions under which arbitrage is not permitted. Colwell (2001) extends the constant volatility one-factor HJM model ${ }^{10}$ to a jump diffusion process and discusses the assumptions under which option prices have explicit formulas.

Although HJM models are flexible enough to contain various term structure models as special cases, they are not without limitations. The main restriction comes from the fact that the larger the number of factors in the term structure model, the greater are the estimation and implementation problems. Only certain cases of one-factor HJM models are Markov ${ }^{11}$. Therefore, in multi-factor HJM models and in most cases of one-factor HJM models, the multinomial and binomial trees, which are often used for fitting the model, may not recombine and computational efficiency can be low.

Short rate models can be represented as one-factor HJM models. Specific volatility functions in the one-factor HJM models lead to certain short rate models that are popular in

\footnotetext{
${ }^{9}$ An excellent overview of the popular short rate models in the HJM terms can be found on pages 149-158 of Baxter and Rennie (1998).

${ }^{10}$ This is the same as Ho and Lee (1986) model.

${ }^{11}$ Carverhill (1994), Jeffrey (1995), Ritchken and Sankarasubramanian (1995), Bliss and Ritchken (1996), and Inui and Kjima (1998) impose certain restrictions on the volatility structure of the HJM models to assure that HJM models are Markovian.
} 
the market. As mentioned in Yan (2001) ${ }^{12}$ and Chapman and Pearson (2001) ${ }^{13}$, most of the recent academic literature focuses on one-factor models.

As documented in Litterman and Scheinkman (1991) $88 \%$ of the variation in U.S. Treasury rates can be explained by the first factor, generally believed to be the level of interest rates. Similar to Litterman and Scheinkman, Chapman and Pearson (2001) carry out principal component analysis on weekly changes in Treasury yields, and support the findings of Litterman and Scheinkman. Chapman and Pearson find that three factors explain 99\% of yield curve variation but first factor alone explains $88 \%$ of variation.

Flesaker (1993) tests the performance of a one-factor constant volatility HJM model to explain the cross-sectional variation in daily market prices of Eurodollar options. Flesaker finds that short-term options are overvalued relative to long term options, exactly opposite of the mean reversion features of Vasicek (1977) and Cox, Ingersoll, and Ross (1985) models. Flesaker suggests that one should allow a humped volatility forward rate term structure. Mercurio and Moraleda (2000) develop a volatility function for instantaneous forward rates that provides a humped volatility structure in the HJM framework. They compare this model with an exponential decay volatility HJM model by estimating implied volatilities using caps and floors. The pricing errors of the humped volatility HJM model are found to be less than those of the exponential decay HJM model. Bühler et al (1999) analyze the pricing errors of two one-factor and two two-factor HJM models, a spot rate model and two two-factor spot rate models using German data on interest rate warrants. They find that the model with lowest absolute deviation is the linear-proportional one-factor HJM model.

One-factor HJM models are also used to estimate implied volatilities. Amin and Morton (1994) find implied interest rate volatilities of six one-factor HJM models using Eurodollar options. They consider the Ho and Lee (1986), square root, proportional, linear absolute, exponential decay and linear proportional models. The first three are one-parameter and the last three are two-parameter models. They find that two-parameter models fit prices better but one-parameter estimates are more stable. Among one-parameter models, the Ho and

\footnotetext{
${ }^{12}$ Yan (2001) reviews dynamic default term structure models.

${ }^{13}$ Chapman and Pearson (2001) discuss the estimation of term structure models and provide an excellent review of recent advances in research related to short rate models.
} 
Lee model performs better. Among two-parameter models, the linear proportional model is better. Amin and Ng (1997) estimate implied volatilities from Eurodollar options prices by incorporating different spot rate models in the HJM framework ${ }^{14}$. They find that these implied volatility estimates explain most of the realized interest rate volatility. They also find that, the Vasicek (1977) and linear proportional models perform better than others. Moraleda and Pelsser (2000) compare three spot rate models and three Markov forward rate models using three-month caps and floors ${ }^{15}$. They find that spot rate models provide a better fit to the cap and floor prices than the forward rate models.

Gupta and Subrahmanyam (2000) use one-factor models to analyze convexity bias in pricing interest rate swaps. The convexity bias is the upward bias in the implied swap rates that is a result of using Eurodollar futures rates that are higher than the forward rates that should be used to determine the swap rates. They consider the Vasicek, Cox-Ingersoll-Ross, Hull-White, and Black-Karasinski models. They also consider a two-factor HJM model. Convexity curves are similar for the Hull-White, and Black-Karasinski models. The Vasicek model produced a convexity curve close to that of the Hull-White model for up to three years of maturity.

Since one-factor models are still widely used and short rate models can be represented as one-factor HJM models, we focus on one-factor HJM models. We consider constant volatility (Ho and Lee (1986)) and exponential decay volatility models (Vasicek (1977)), a constant decay volatility model used in $\mathrm{Au}$ and Thurston (1995), and the humped volatility model proposed by Mercurio and Moraleda (2000).

\subsubsection{Interest Rate Measures Derived from Specific HJM Models}

$\mathrm{Au}$ and Thurston (1995) develop duration measures for certain one-factor HJM models. Assuming that all bond prices are functions of the spot rate, Au and Thurston derive

\footnotetext{
${ }^{14}$ The spot rate models incorporated in the HJM framework are Courtadon (1982), Cox, Ingersoll, and Ross (1985), Ho and Lee (1986), Vasicek (1977) and the linear proportional model of Amin and Morton (1994).

${ }^{15}$ The spot rate models considered in the study are Hull and White (1994), Black and Karasinski (1991) and Pelsser (1997). They use Li et. al. (1995) characterization for Markovian forward rate models and they analyze forward rate models corresponding to Hull and White (1994), square root model and a proportional volatility model.
} 
the bond price process of the forward rate dynamics of one-factor HJM models. The resulting expression shows that the basis risk of a coupon bond derived from an HJM model is a function of the forward rate volatility. Using this basis risk expression, $\mathrm{Au}$ and Thurston derive the corresponding duration measure for a coupon bond. The HJM duration measure developed by $\mathrm{Au}$ and Thurston is a function of forward rate volatility, coupon payments and the zero coupon bond price. Since the HJM duration is a function of forward rate volatility, HJM duration measures differ for different volatility functions. Au and Thurston specifically derive the duration measures for constant, constant decay, and exponential decay volatility structures.

Jeffrey (2000) also shows the relation of duration measures to the forward rate volatility structure of HJM models. Jeffrey provides schemes to determine duration measures for single-factor HJM models. Although the approach of Jeffrey is different than that of Au and Thurston, both arrive at the same HJM duration measure. Jeffrey derives the stochastic process of a certain maturity asset and liability by assuring that the asset-liability portfolio is self-financing. Then Jeffrey matches the basis risk of the asset to the basis risk of the liability. Replacing the asset portfolio with a zero coupon bond maturing at a specific date provides the duration measure for the one-factor HJM models.

Although duration measures of certain one-factor HJM models are available in the literature, the convexity measures of one-factor HJM models are not available in the literature. Therefore, we extend the approach of $\mathrm{Au}$ and Thurston and derive the convexity measures of one-factor HJM models. Fruhwirth (2001) also follows a similar procedure and arrives at the same convexity measures with ours.

\subsection{Empirical Evidence on the Performance of Alternative Interest Rate Risk Measures}

Theoretically, Macaulay's duration is accurate under a flat term structure and parallel shifts, and Fisher-Weil duration is a valid risk measure for parallel shifts. Empirical evidence, however, is mixed on the performance of these measures. 
The first empirical work on the effectiveness of traditional risk measures is Fisher and Weil (1971). The amount of research on this area accelerated after this work, extended by additional theoretical developments on alternative risk measures and term structure models.

Fisher and Weil (1971) uses annual corporate bond yield data of Durand (1942) to analyze the effectiveness of different immunization strategies. Low risk corporate bonds for the years 1925-1968 are used for the analysis. Fisher and Weil assume that yield curve shifts are parallel, and simulate returns for holding periods of 5, 10, and 20 years. These simulated portfolios are compared with a maturity matching bond strategy. The immunization strategy is to use Fisher-Weil duration for matching the duration of a two coupon bond portfolio with that of a target bond with a promised yield to maturity. The returns of the duration-matched portfolio are found to be closer to the promised return than the returns of the maturity strategy. The effectiveness of a duration matching strategy relative to a maturity strategy is greater the longer the holding period.

Using Durand yield data for the period 1925-1978, Bierwag, Kaufman, Schweitzer, and Toevs (1981) also analyze the effectiveness of immunized portfolios relative to the maturity bond portfolio. Barbell portfolios that consist of a maturity bond and a 20-year bond. both with a 5 percent coupon, are used in the simulations. The holding period is 10 years. Four alternative duration measures are tested: Macaulay's duration, the duration measures of Bierwag (1977) for multiplicative and additive shocks, and the duration measure developed by Khang (1979) for maturity-dependent multiplicative shocks. Similar to Fisher and Weil, the returns of the immunized portfolios are compared with the promised return. Macaulay's duration, and both Bierwag durations perform better than the maturity strategy, whereas Khang's duration measure performs poorly. Bierwag's additive duration measure is found to outperform the others.

Cox, Ingersoll, and Ross (1979) (CIR, henceforth) estimate the parameters of their proposed term structure model by using weighted least squares on weekly auction rates of 91day Treasury bills over the period 1967 to 1976. Simulation is used to compare Macaulay's duration, Fisher-Weil duration, and the proposed stochastic duration. Holding periods considered are $5,10,15,20,35$, and 50 years. Under the assumption that interest rates follow 
a driftless random walk, Fisher-Weil duration is found to be more accurate. When mean reversion is considered, however, both the Macaulay and the Fisher-Weil durations are found to perform poorly compared to stochastic duration.

Brennan and Schwartz (1983) develop a two-factor model to describe the interest rate process. These two factors are the spot rate and the yield on a consol bond. The proposed hedging strategy is to construct the bond portfolio such that its estimated response to the changes in the spot rate and consol yield will be the same as for a discount bond with the appropriate maturity. This proposed hedging strategy is compared to the Fisher-Weil duration using monthly U.S. Government Bond data from December 1958 to December 1979. The holding periods are five and ten years. The empirical evidence shows that the Fisher-Weil duration performs as well as the proposed hedging strategy. The authors suggest that the Fisher-Weil duration is more appropriate for hedging of default-free bonds.

Nelson and Schaefer (1983) develop a two-factor model where the factors are a short rate and the spread between a long and a short rate. They compare the immunization performance of the risk measure developed from this two-factor model to the Fisher-Weil duration. The data used in the study are the CRSP U.S. Government Bond data from December 1925 to December 1979. Holding periods are 5, 10, and 15 years. The empirical results of the study suggests that the Fisher-Weil duration performs as well as, and in some cases, even better than, the proposed risk measure developed from the two-factor model.

Gultekin and Rogalski (1984) test the implications of alternative duration measures and compare the performances of these measures with the stochastic duration of Cox, Ingersoll, and Ross (1979). Alternative duration measures tested in the study are the FisherWeil duration, the Bierwag (1977) duration for multiplicative yield curve shifts, a duration for additive and multiplicative yield curve shifts developed by Cooper (1977), and Khang (1979) maturity-dependent additive and multiplicative shifts. Gultekin and Rogalski test three hypotheses: The relation between bond price changes and duration is linear; duration is a complete risk measure; and capital markets for bonds are efficient. They use monthly CRSP Government Bond Data from January 1947 to December 1976. Holding periods are five and ten years. The results suggest that the Fisher-Weil duration overstates the basis risk for long- 
term bonds. None of the alternative duration measures other than the stochastic duration of CIR performs better than the Fisher-Weil duration. None of these duration measures explain price volatility caused by yield shape changes better than the others. For stochastic duration, the parameter values given by CIR are used, and the liquidity premium is assumed to be zero. Stochastic duration is also not substantially superior to other durations as a measure of basis risk. All the tested hypotheses are rejected for all duration models, and the authors conclude that immunization strategies based on these measures may not work.

Bierwag, Kaufman, Latta, and Roberts (1987) criticize the Gultekin and Rogalski (1984) study due to biases induced in the empirical tests. One criticism is that unless the yield change is the same across all maturities, the regression of Gultekin and Rogalski is misspecified. Moreover, convexity when dealing with non-infinitesimal changes may lead to the over and underestimation of duration. The stratification of cross-sectional data according to time period, rather than maturity may lead to biases in the empirical tests. Also, the underlying stochastic process is not known for certain. Misspecification of the stochastic process will affect the performance of the duration measures. Any of these reasons might be driving the results of Gultekin and Rogalski, and therefore, it is not reasonable to conclude that duration measures do not work in immunization. Bierwag et al also replicates a similar study of Gultekin and Rogalski by using the data from Data Resources Inc., which consists of daily offer quotes by Bank of America on all Treasuries. The data are from May 1980 to 1985, and the holding period considered by Bierwag et al is five years. The results do not favor stochastic duration of CIR over the alternatives.

Ilmanen (1992) examines how much of the cross-sectional variation in bond returns can be explained by duration. Ilmanen finds that duration explains $80 \%$ to $90 \%$ of the return variance for U.S. government bonds, and the explanatory power of duration increases over time. The findings of Ilmanen are consistent with those of Litterman and Scheinkman (1991) and Chapman and Pearson (2001). These two studies use three-factor principal component analysis and find that the first factor, generally thought to be the level of interest rates, explains almost $90 \%$ of the variation in U.S. Treasury rates. 
Ho, Cadle and Theobald (2001) test the hedging performance of three alternative methods. One of them is a delta-neutral hedge suggested by Jarrow and Turnbull (1994), which is based on a generalized method of moments estimation of exponential decay volatility parameters of a one-factor HJM model. The other two are a spot rate sensitivity-based hedge and a modified duration hedge. Ho et al use weekly prices of three-month Sterling futures contracts from December 18, 1991 to December 16, 1998. Three-month LIBOR is used as a proxy for the spot rate. Ho et al hedge one-year Sterling futures positions with two-year Sterling futures contracts. The results suggest that a delta-hedging strategy is superior to spotrate sensitivity-based hedging but is not superior to modified duration hedging in all cases.

\subsection{Portfolio Formation Strategies}

Redington (1952) introduced the term immunization to the finance literature in a study in which he developed Macaulay's duration, calling it the mean term ${ }^{16}$. Immunization is defined as the condition in which a portfolio is insensitive to interest rate changes. An alternative definition of immunization is later introduced by Fisher and Weil (1971). According to Fisher and Weil, a bond portfolio is immunized against interest rate changes if the holding period return of the portfolio is at least as large as the target return ${ }^{17}$.

To immunize a given coupon bond, or a given bond portfolio, there are many alternative portfolios that can be formed to match the immunization criteria. In this study, we consider the commonly used bullet, barbell, random, and minimum M-square portfolios.

In the following subsections, we first survey the literature on random portfolios. Then, we review the literature on bullet and barbell portfolios. The last subsection is devoted to the research on minimum M-square portfolios.

\footnotetext{
${ }^{16}$ This nomenclature seems to correspond with the common perception of duration as an average maturity of a bond, wherein a coupon bond is viewed as equivalent to a portfolio of zero coupon bonds. This perception is valid only for option-free and default-free bonds.

${ }^{17}$ Fisher-Weil's definition arises out of the convex relationship between the bond price and interest rates. Much of the subsequent theory of immunization is based on exploiting this result.
} 


\subsubsection{Random Portfolios}

Empirical studies that test the performance of alternative duration measures differ in the method of the portfolio formation. Gultekin and Rogalski (1984), and Bierwag, Kaufman, Latta, and Roberts (1987) form portfolios with a random drawing of securities that matches the duration of the target portfolio. The number of securities in these portfolios varies from two to ten in Gultekin and Rogalski, and up to six in Bierwag et al. Nevertheless, in these studies, the performance of different portfolio formations cannot be analyzed. One portfolio formation can be superior to the other under certain circumstances, and the performance of a certain portfolio strategy cannot be inferred from the average performance of the random portfolios.

\subsubsection{Bullet and Barbell Portfolios}

In an immunization strategy, a barbell portfolio strategy is the duration-matched portfolio that consists of bonds with the highest and lowest durations available. A bullet portfolio strategy, on the other hand, is the duration-matched portfolio with bonds that have the closest durations to the target duration. Suppose we have a set of bonds to form a portfolio with a duration of 5 years. Also suppose that among the available bonds, the two bonds that has the closest duration to the target of 5 years is 4.5 and 5.5 years. Then we choose these two bonds to form the bullet portfolio. Suppose that among the bonds, the one with the lowest duration has a duration of 4 years and the one with the highest duration has a duration of 20 years. Then we choose these two bonds to form the barbell portfolio.

In the duration literature, a barbell portfolio is commonly used. Fisher and Weil (1971) form immunizing portfolios from two coupon bonds where the short-term coupon bond matures at the end of the holding period ${ }^{18}$. The coupon rate of these bonds is set to $4 \%$. Fisher and Weil use one-, five-, ten-, and twenty-year holding periods. Bierwag, Kaufman, Schweitzer, and Toevs (1981) also use barbell portfolios to test the performance of alternative duration measures. A ten-year holding period is used for the analysis, and the coupon rate of

\footnotetext{
${ }^{18}$ Due to the coupon payments, the duration of the short term coupon bond will be less than the duration of the corresponding zero-coupon bond.
} 
the bonds is set to $5 \%$. A coupon bond that matures at the end of the holding period and a twenty-year coupon bond are used in the portfolios.

Leibowitz (1980) assumes yield curve shifts are parallel and compares the performances of different immunization strategies for a sample period of January 1958 to December 1979. Performance is measured as the proximity of the average realized returns of the bond portfolio over the holding period to the holding period return of the target bond. For a holding period of five years, Leibowitz shows that the worst performer is a barbell portfolio constructed from one-year and thirty-year bonds. The next worst performer is found to be a bullet portfolio.

Fuller and Settle (1994) analyze the importance of the determinants of duration. Duration is a function of the yield to maturity, coupon rate, and term to maturity. Fuller and Settle find that among these determinants, the coupon rate is the least important determinant of the duration for a coupon rate higher than $2 \%$ or $3 \%$. For short maturity bonds, with terms to maturity less than five years, the term to maturity is the most important factor, and the yield to maturity is not very important. For bonds with maturity greater than ten years, yield to maturity is the most important factor, and term to maturity is not particularly important. For bonds with intermediate terms to maturity, both yield to maturity and term to maturity are important factors. The results of this study suggest that the coupon rate is not particularly important in constructing an immunized portfolio. To duration match long term bonds, it may be more appropriate to select bonds that have a yield to maturity close to that of the target portfolio, rather than selecting bonds that have term to maturity close to that of the target portfolio. For a short maturity target portfolio, it would be more appropriate to form a bullet portfolio. For intermediate maturity target portfolios, a portfolio that has a yield to maturity closest to the target portfolio may be a more appropriate immunization strategy.

Chance and Jordan (1996) examine the percentage of default-free bond returns explained by duration, convexity, theta ${ }^{19}$, and the interaction of theta and duration. Their results suggest that the duration approximations of the actual bond returns overestimate the

\footnotetext{
${ }^{19}$ Theta is the change in the bond price with respect to change in time. It is also called time decay in Jarrow (1996, p. 114). Bond practitioners typically call it "pull to par", reflecting the characteristic that a bond's price converges to par value at maturity.
} 
bond returns, especially for short-maturity bonds. The theta term, however, corrects this overestimation. The convexity term does not add anything significant to the approximation of the short-maturity bond returns, but is relatively more important for the approximation of long-maturity bonds returns. The theta factor contributes more than the convexity factor to the bond return approximation for both short and long maturity bonds. The interaction factor, however, is found to be more important than convexity for short maturity bonds, but the inverse is true for long maturity bonds.

Ilmanen and Iwanowski (1997) analyze alternative immunization strategies under different dynamics of the yield curve. The curvature of the yield curve can lead to different performances between bullet and barbell strategies. If the shape of the yield curve is humped, a barbell portfolio's convexity advantage is expected to offset a bullet portfolio's yield advantage. In this case, there should not be any difference in the performances of bullet and barbell strategies. Historically, however, it is found that bullet portfolios have slightly higher returns than barbell portfolios. Ilmanen and Iwanowski explain this difference by the lower return volatility of the barbell portfolios.

\subsubsection{Minimum M-Square Portfolios}

Fong and Vasicek (1983) introduce a high order measure, which they call M-square. M-square can be considered as a weighted variance of time to payments around the holding period date where the weights are the present values of the payments. Fong and Vasicek state that minimizing the M-square of a duration-matched portfolio will immunize the portfolio against yield curve shifts that cannot be immunized by the selected duration measures.

After introducing the concept of M-square, Fong and Vasicek compare a deepdiscount bond with a barbell portfolio that has the same duration. Deep-discount bonds that mature close to the holding period have low M-square values. Conversely, in a barbell portfolio, the payments are more disperse, and thus its M-square is relatively high. For parallel shifts in the yield curve, these two portfolios will be affected the same because of the inverse relation between reinvestment risk and price risk. If there is a twist in the term structure, the above argument no longer holds. Fong and Vasicek present a case where short rates go down 
and long rates go up. In this case, there will be a capital loss for both portfolios, but it will affect the barbell portfolio more since its payments must be invested at a lower rate in addition to the capital loss of the remaining payments.

Bierwag, Fooladi, and Roberts (1993) analyze bullet and barbell strategies and their relation to M-square. Bierwag et al show that a bullet portfolio is the minimum M-square portfolio if and only if M-square is a strictly convex function of duration. For a set of bonds with identical coupon rates or for a set of par bonds, this convexity condition is met, and therefore, for a portfolio of these bonds, the minimum M-square portfolio is a bullet portfolio. 


\section{CHAPTER 3}

\section{TRADITIONAL RISK MEASURES}

The traditional risk measures that are mostly referenced in the literature and used by practitioners are duration and convexity. The Macaulay (1938), and Fisher and Weil (1971) duration measures are theoretically accurate only under parallel yield curve shifts. The empirical evidence, however, shows that these risk measures perform well even under nonparallel shifts in the yield curve. Convexity is a higher order risk measure that is particularly important when the yield curve shifts are non-infinitesimal. In addition to these risk measures Fong and Vasicek (1983) develop a risk measure, M-square, to reduce the interest rate risk for non-parallel shifts in the yield curve.

These traditional risk measures are covered in detail in the following subsections. We first examine the characteristics of traditional duration measures. Then we present the convexity measure. The last subsection is on M-square.

\subsection{Duration}

The traditional duration measures considered in the study are Macaulay's duration and Fisher-Weil duration. At time $t$, the price of a coupon bond maturing at $T=\tau_{n}$, with coupon

payments at time periods $\tau_{\mathrm{i}}$ and principal payment at $\tau_{\mathrm{n}}$, is represented as $B(t, T)^{20}$. The duration of this coupon bond, $D(t)$, is as follows:

$$
D(t)=\frac{\sum_{i=1}^{n}\left(\tau_{i}-t\right) C_{i} P\left(t, \tau_{i}\right)}{B(t, T)}
$$

where,

$$
B(t, T)=\sum_{i=1}^{n} C_{i} P\left(t, \tau_{i}\right)
$$

\footnotetext{
${ }^{20}$ All bonds considered in this study do not contain options.
} 
$C_{i}$ : $\quad$ Coupon on payment at date $\tau_{\mathrm{i}}$, where $\mathrm{t} \leq \tau_{1}<\tau_{2}<\ldots<\tau_{\mathrm{n}} \leq T$, for a trading interval of $[\mathrm{t}, \mathrm{T}]$

$P\left(t, \tau_{\mathrm{i}}\right):$ The price at time $t$ of a zero coupon bond maturing at time $\tau_{i \text {. }}$

The duration of a coupon bond is achieved by the rearrangement of the first term of the Taylor series expansion of the bond price around its yield ${ }^{21}$. The definition of $P\left(t, \tau_{\mathrm{i}}\right)$ gives the different definitions of duration. Let $y(t, T)$ be the yield at time $t$ on a $T$-maturity zero-coupon bond. $P\left(t, \tau_{\mathrm{i}}\right)$ can be defined as $\frac{1}{\left[1+y\left(t, \tau_{i}\right)\right]^{\left(\tau_{i}-t\right)}}$ in terms of yield to maturity. If $y(t, T)=y(t)$, the yield to maturity will be the same for all maturity bonds. This implies a flat yield curve, and the duration measure in equation (5) reduces to the Macaulay's duration, $D_{M}(t)$. If the yield to maturity differs for different maturity bonds, we have Fisher-Weil duration, $D_{F W}(t)$. If $y(t, T)$ is the continuous yield, then $P\left(t, \tau_{\mathrm{i}}\right)$ can be defined as $\exp \left(-y\left(t, \tau_{\mathrm{i}}\right)\left(\tau_{\mathrm{i}}-t\right)\right)$. Again, for the constant yield to maturity $y(t, T)=y(t)$, equation (5) gives the continuous time version of Macaulay's duration, $D_{M C}(t)$. For varying yields to maturity for different maturity bonds, equation (5) gives the continuous time version of Fisher-Weil duration $D_{F W C}(t)$. Macaulay's duration measure is theoretically accurate for flat term structures and for additive shifts, whereas Fisher-Weil duration is theoretically accurate for any yield curve structure but only for additive shifts.

A slight variation of Macaulay's duration is the modified duration. Modified duration, $M D(t)$, is obtained by dividing the discrete time Macaulay's duration by one plus the yield to maturity. So, $M D(t)=D_{M}(t) /(1+y(t))$. Modified duration is applicable only for discrete time duration measures ${ }^{22}$.

In this study, forward rates and volatilities are estimated in continuous time. Therefore, the continuous yield is used in the duration measures. For completeness, both discrete and continuous duration measures are summarized below.

\footnotetext{
${ }^{21}$ See Appendix 1 for derivation.
} 


\subsubsection{Summary of Duration Measures}

Macaulay's Duration (Discrete) $\left(D_{M D}\right)$ :

$D_{M D}(t)=\frac{1}{B(t, T)} \sum_{i=1}^{n} C_{i}\left(\tau_{i}-t\right)[1+y(t)]^{-\left(\tau_{i}-t\right)}$

Macaulay's Duration (Continuous) $\left(D_{M C}\right)$ :

$D_{M C}(t)=\frac{1}{B(t, T)} \sum_{i=1}^{n} C_{i}\left(\tau_{i}-t\right) e^{-y(t)\left(\tau_{i}-t\right)}$

Fisher-Weil Duration (Discrete) $\left(D_{F W D}\right)$ :

$D_{F W D}(t)=\frac{1}{B(t, T)} \sum_{i=1}^{n} C_{i}\left(\tau_{i}-t\right)\left[1+y\left(t, \tau_{i}\right)\right]^{-\left(\tau_{i}-t\right)}$

Fisher-Weil Duration (Continuous) $\left(D_{F W C}\right)$ :

$D_{F W C}(t)=\frac{1}{B(t, T)} \sum_{i=1}^{n} C_{i}\left(\tau_{i}-t\right) e^{-y\left(t, \tau_{i}\right)\left(\tau_{i}-t\right)}$

Modified Duration $(M D)$ :

$M D(t)=\frac{1}{B(t, T)(1+y(t))} \sum_{i=1}^{n} C_{i}\left(\tau_{i}-t\right)[1+y(t)]^{-\left(\tau_{i}-t\right)}$

\subsection{Convexity}

For non-infinitesimal yield curve changes, convexity provides an improvement to capture the yield curve movement and to provide a better hedge. Convexity is derived from the second derivative of the coupon bond's price with respect to the yield ${ }^{23}$.

\footnotetext{
${ }^{22}$ The relation between bond prices and duration measures is given in Appendix 1.

${ }^{23}$ See Appendix 1 for derivation.
} 
The discrete version of convexity can be derived by considering the zero-coupon bond price as $P\left(t, \tau_{\mathrm{i}}\right)=\frac{1}{\left[1+y\left(t, \tau_{i}\right)\right]^{\left(\tau_{i}-t\right)}}$. In this case, convexity of a coupon bond, $\operatorname{Conv}_{D}(t)$ can be represented as follows:

$$
\begin{aligned}
\operatorname{Conv}_{D}(t) & =\frac{1}{\sum_{i=1}^{n} C_{i} P\left(t, \tau_{i}\right)} \sum_{i=1}^{n} \frac{C_{i}\left(\tau_{i}-t\right)\left(\tau_{i}-t+1\right)}{\left[1+y\left(t, \tau_{i}\right)\right]^{\left(\tau_{i}-t+2\right)}} \\
& =\frac{1}{B(t, T)} \sum_{i=1}^{n} \frac{\left(\tau_{i}-t\right)\left(\tau_{i}-t+1\right) C_{i} P\left(t, \tau_{i}\right)}{\left(1+y\left(t, \tau_{i}\right)\right)^{2}}
\end{aligned}
$$

Similar to the duration measure, yield to maturity can be assumed constant, $y(t, T)=y(t)$, as in Macaulay's duration, or varying for different maturity bonds, as in FisherWeil duration. Constant discrete yield convexity is referred as $\operatorname{Conv}_{M D}(t)$, and varying discrete yield convexity is referred as $\operatorname{Conv}_{F W D}(t)$.

The continuous version of convexity can be derived by defining the zero coupon bond price $P\left(t, \tau_{\mathrm{i}}\right)$ as $\exp \left(-y\left(t, \tau_{\mathrm{i}}\right)\left(\tau_{\mathrm{i}}-t\right)\right)$. The resulting convexity can be represented as follows:

$$
\begin{aligned}
\operatorname{Conv}_{C}(t) & =\frac{1}{\sum_{i=1}^{n} C_{i} P\left(t, \tau_{i}\right)} \sum_{i=1}^{n} C_{i}\left(\tau_{i}-t\right)^{2} e^{-y\left(t, \tau_{i}\right)\left(\tau_{i}-t\right)} \\
& =\frac{1}{B(t, T)} \sum_{i=1}^{n} C_{i}\left(\tau_{i}-t\right)^{2} P\left(t, \tau_{i}\right)
\end{aligned}
$$

Constant continuous yield convexity and varying continuous yield convexity are referred as $\operatorname{Conv}_{M C}(t)$, and $\operatorname{Conv}_{F W C}(t)$, respectively.

In this study the continuous convexity measures are used with the continuous duration measures. For completeness, we give the formulas for convexity measures for both constant and varying discrete and continuous yields. 


\subsubsection{Summary of Convexity Measures}

Discrete Constant Yield Convexity $\left(\operatorname{Conv}_{M D}\right)$ :

$\operatorname{Conv}_{M D}(t)=\frac{1}{B(t, T)} \sum_{i=1}^{n} C_{i}\left(\tau_{i}-t\right)\left(\tau_{i}-t+1\right)[1+y(t)]^{-\left(\tau_{i}-t\right)}$

Continuous Constant Yield Convexity (Conv $\left.{ }_{M C}\right)$ :

$\operatorname{Conv}_{M C}(t)=\frac{1}{B(t, T)} \sum_{i=1}^{n} C_{i}\left(\tau_{i}-t\right)^{2} e^{-y(t)\left(\tau_{i}-t\right)}$

Discrete Varying Yield Convexity $\left(\operatorname{Conv}_{F W D}\right)$ :

$\operatorname{Conv}_{F W D}(t)=\frac{1}{B(t, T)} \sum_{i=1}^{n} C_{i}\left(\tau_{i}-t\right)\left(\tau_{i}-t+1\right)\left[1+y\left(t, \tau_{i}\right)\right]^{-\left(\tau_{i}-t\right)}$

Continuous Varying Yield Convexity $\left(\operatorname{Conv}_{F W C}\right)$ :

$\operatorname{Conv}_{F W C}(t)=\frac{1}{B(t, T)} \sum_{i=1}^{n} C_{i}\left(\tau_{i}-t\right)^{2} e^{-y\left(t, \tau_{i}\right)\left(\tau_{i}-t\right)}$

\subsection{M-Square}

M-square was developed by Fong and Vasicek (1983) as a measure of immunization risk against arbitrary interest rate changes, i.e. parallel as well as non-parallel shifts. Like convexity, this measure is based on second order conditions of yield curve changes. When the bond price is approximated around duration by a Taylor series expansion, a rearrangement of terms gives the change in the bond price as a function of the M-square.

For a planning horizon $T$, and a target zero coupon bond $P(\mathrm{t}, T)$, the value of a duration matched immunization portfolio I(t), can be defined as follows:

$I(t)=\sum_{i=1}^{n} C_{i} P\left(t, \tau_{i}\right)$

s.t. 
Duration of the portfolio with value $I(t)=T-t$

If there is no change in the forward rates, the values of the immunization portfolio at time $T$ will be $I(T)=I(0) / P(0, T)$. For a given change in the instantaneous spot rate $\Delta f(t, t)$, let the change in the horizon date value of the immunization portfolio be $\Delta I(T)$. Fong and Vasicek (1983) show the relation between the change in the immunization portfolio and the M-Square as follows:

$$
\frac{\Delta I_{T}}{I_{T}}=-M^{2} \Delta_{S}
$$

where

$$
\begin{aligned}
& M^{2}(t)=\sum_{t=1}^{n} \frac{\left(\tau_{i}-t-T\right)^{2} C_{i} P\left(t, \tau_{i}\right)}{\sum_{t=1}^{n} C_{i} P\left(t, \tau_{i}\right)} \\
& \Delta_{S}=\frac{1}{2}\left(\frac{d(\Delta f(t, t))}{d t}-(\Delta f(t, t))^{2}\right)_{t=T}
\end{aligned}
$$

In the above equations, $\Delta f(t, t)$ represents the change in the instantaneous spot rate, and $\Delta_{S}$ is a quadratic measure of the departure of the term structure shift from a parallel type. Although $\Delta_{S}$ depends on the interest rate movement, M-square depends only on the portfolio characteristics. Therefore, to increase the likelihood of an immunized portfolio, M-square is minimized subject to the duration condition. M-square can be seen as a time-to-payment variance around the bond cash flow dates. M-square is zero for a zero coupon bond with duration $D$, and positive for coupon bonds when there is no shortselling. M-square is especially important if the shift is not parallel. Minimizing M-square reduces the effect of a non-parallel shift on a duration-matched portfolio. Fong and Vasicek introduce M-square as a measure of immunization risk since it captures some of the exposure of a portfolio to interest rate movements. 
Nawalkha and Lacey (1990) derive closed-form solutions for M-square as well as some higher order durations. They show that first and second order duration measures of Bierwag (1987) are related to M-square $\left(M^{2}=D_{2}-2 T D_{1}+T^{2}\right)$, where $D_{1}$ and $D_{2}$ are first and second order duration measures of Bierwag (1987), and $T$ is the holding period. 


\section{CHAPTER 4}

\section{THE HEATH-JARROW-MORTON FRAMEWORK}

Heath, Jarrow, and Morton (1992) developed an arbitrage-free pricing framework for capturing the evolution of forward rates, and therefore modeling the term structure of interest rates. The assumption of different volatility functions and different factor specifications give different term structure models. The general HJM is a multi-factor model. An n-factor HJM model specifies the evolution of the instantaneous forward rate according to the below stochastic process:

$$
d f(t, T)=\alpha(t, T, w) d t+\sum_{i=1}^{n} \sigma_{i}(t, T, w) d W_{i}(t)
$$

where,

$f(t, T): \quad$ Instantaneous forward rate at time $t$ for date $T, T>t$

$W_{t}: \quad$ Brownian motion at time $t$,

$\alpha(t, T, w)$ : Random drift term of the forward rate curve where $T>t$, and $w$ is a sample point defined on $\Omega$,

$\sigma_{i}(t, T, w)$ : Stochastic volatility function $\mathrm{i}$, for $\mathrm{i}=1, . . \mathrm{n}$, where $T>t$, and $w$ is a sample point defined on $\Omega$.

For a given initial forward rate curve $f(0, T)$, the evolution of the forward rate can be represented as an integral of equation (9):

$$
f(t, T)=f(0, T)+\int_{0}^{t} \alpha(v, T, w) d v+\sum_{i=1}^{n} \int_{0}^{t} \sigma_{i}(v, T, w) d W_{i}(v)
$$

Following from equation (10), the spot rate, $r(t)$ follows the equation: 


$$
r(t)=f(0, t)+\int_{0}^{t} \alpha(v, t, w) d v+\sum_{i=1}^{n} \int_{0}^{t} \sigma_{i}(v, t, w) d W_{i}(v)
$$

The no-arbitrage condition puts a restriction on the relation of the drift to the volatilities. This so-called forward rate drift condition is as follows:

$$
\alpha(t, T, w)=\sum_{i=1}^{n} \sigma_{i}(t, T, w) \int_{t}^{T} \sigma_{i}(t, v, w) d v-\sum_{i=1}^{n} \sigma_{i}(t, T, w) \lambda_{i}(t)
$$

In the above equation, $\lambda_{i}$ must be independent of the maturity of a particular bond. Setting $\lambda_{\mathrm{i}}$ equal to zero transforms the probability measure $\mathrm{P}$ to an equivalent martingale measure Q. Therefore, under an equivalent martingale measures, the drift restriction is as follows:

$$
\alpha(t, T, w)=\sum_{i=1}^{n} \sigma_{i}(t, T, w) \int_{t}^{T} \sigma_{i}(t, v, w) d v
$$

When we replace the drift in the forward rate dynamics in equation (10), with the above equation, we get the stochastic process of the forward rate under the equivalent martingale measures. This stochastic process can be represented as follows:

$$
d f(t, T)=\left(\sum_{i=1}^{n} \sigma_{i}(t, T, w) \int_{t}^{T} \sigma_{i}(t, v, w) d v\right) d t+\sum_{i=1}^{n} \sigma_{i}(t, T, w) d \widetilde{W}_{i}(t)
$$

In equation (14), $\widetilde{W}(t)$ corresponds to the Wiener process under the equivalent martingale measure. As it can be observed, under equivalent martingale measures, the market price of risk disappears and the forward rate dynamics are a function of forward rate volatilities only. In this respect, in the HJM models, different volatility functions correspond 
to different HJM models. For example, for one-factor HJM models, if the volatility is constant, $\sigma(t, T)=\sigma$, we have the Ho and Lee (1986) model. If the volatility is an exponentially decaying, $\sigma(t, T)=\sigma \exp (-\lambda(T-t))$, we have the extended-Vasicek Hull and White (1993). Other short rate models can also be represented as special cases of one-factor HJM models.

The price at time $t$, for a zero coupon bond maturing at time $T, P(t, T)$ is determined by the forward rate structure. The forward rates also can be represented as functions of zero coupon bond prices. These relations are given below.

$$
\begin{aligned}
& P(t, T)=\exp \left(-\int_{t}^{T} f(t, u) d u\right) \\
& f(t, T)=-\frac{\partial \log P(t, T)}{\partial T}
\end{aligned}
$$

In HJM models, a money market account plays the important role of a numeraire. The price of the money market account initialized at time 0 with a unit money is represented as $B(t)$. As shown in the following equation, the price of this money market account is a function of the spot rate.

$$
B(t)=\exp \left(\int_{0}^{t} r(u) d u\right)
$$

The relative price of a zero coupon bond, $Z(t, T)=P(t, T) / B(t)$, or $P(t, T) \exp \left(-\int_{0}^{t} r(u) d u\right)$, becomes a martingale under the equivalent martingale measure. This is, of course, classic and well-known result that arises from the requirement that arises from the requirement that there are no arbitrage opportunities. 
In this study, we focus on one-factor HJ M models. In one-factor HJM models, there is only one random factor driving the forward rate dynamics. Therefore bond prices of different maturities are perfectly correlated. On the other hand, one-factor models are still popular in the industry and widely used in the academic literature. 


\section{CHAPTER 5}

\section{INTEREST RATE RISK MEASURES OF ONE-FACTOR HEATH-JARROW-MORTON MODELS}

In this study, one-factor HJM models are analyzed. The dynamics of the forward rate in a one-factor HJM model can be represented by the stochastic process below:

$$
\mathrm{d} f(t, T)=\alpha(t, T, \omega) \mathrm{dt}+\sigma(t, T, \omega) \mathrm{d} W_{t}
$$

After the specification of the volatility functions, the resulting term structure model provides the bond pricing framework, which leads to the delta and gamma of the bond, which then can be mapped onto the alternative measures for duration and convexity. In the following sections we first analyze the duration measures of one-factor HJM models. Then we derive the convexity measures of one-factor HJM models.

\subsection{Duration Measures of One-Factor HJM Models}

The delta of the bonds can be found by taking the first derivatives, respectively, of the bond price with respect to the instantaneous spot rate, $f(t, t)$. Au and Thurston (1995) derive the duration measures of certain continuous-time one-factor HJM term structure models. Jeffrey (2000) shows an alternative approach to determine duration and convexity measures for single factor HJM models. In this study, we follow Au and Thurston (1995). Following their framework, the delta of a coupon bond can be represented as follows:

$$
\frac{\partial B(t, T)}{\partial f(t, t)}=-\sum_{i=1}^{n} C_{i} \frac{P\left(t, \tau_{i}\right)}{\sigma(t, t, w)} \int_{T}^{\tau_{I}} \sigma(t, v, \omega) d v
$$


This delta measure of a coupon bond can be mapped onto the duration. The basis risk of a coupon bond, $-\frac{\partial B(t, T)}{\partial f(t, t)} / B(t, T)$, will give the duration measure, $D_{H J M}(t)$, of the bonds in the HJM framework:

$$
D_{H J M}(t)=\frac{\sum_{i=1}^{n} C_{i}\left(\int_{T}^{\tau_{l}} \sigma(t, v, \omega) d v\right) P\left(t, \tau_{i}\right)}{\sigma(t, t, \omega) \sum_{i=1}^{n} C_{i} P\left(t, \tau_{i}\right)}
$$

$\mathrm{Au}$ and Thurston show some examples of the above duration measure for constant, exponentially decaying, constant decaying, and constant maturity volatility functions. For constant volatility, $\sigma(t, T, \omega)=\sigma$, and $D_{H J M}(t)$ reduces to equation (5), which is the traditional duration measure. For exponentially decaying volatility, the delta of the bond derived from equation (19) gives the delta presented by Jarrow and Turnbull (1996, p. 493). Thus, for this special case, $D_{H J M}(t)$ reduces to the delta given by Jarrow and Turnbull (1996), divided by the bond price.

Following Amin and Morton (1994), we considered only the one-factor HJM models that have a time-invariance property. The volatility functions of these HJM models depend only on time to maturity, not on the calendar date ${ }^{24}$. In this respect, constant, constant decay, and exponential decay volatility functions analyzed by $\mathrm{Au}$ and Thurston are considered. Additionally, the volatility function developed by Mercurio and Moraleda (2000) is chosen as the fourth one-factor HJM model. Mercurio and Moraleda develop a volatility function to represent the humped term structure of volatility observed in the market. This humped volatility function is as follows:

$$
\sigma(t, T, \omega)=\sigma(1+\gamma(T-t)) e^{-\lambda(T-t)}
$$

\footnotetext{
${ }^{24}$ Since only a restricted number of one-factor HJM models are considered, it seems more appropriate to select the models so that they have common properties. The study can then be extended to cover calendar time dependent volatility functions.
} 
We derived the duration measure of this one-factor HJM model with humped volatility. This duration measure is as follows:

$$
D_{H J M}(t)=\frac{1}{\sum_{i=1}^{n} C_{i} P\left(t, \tau_{i}\right)} \sum_{i=1}^{n} C_{i} P\left(t, \tau_{i}\right) \frac{\gamma}{\lambda}\left[\left(\frac{1}{\gamma}+\frac{1}{\lambda}\right)\left(1-e^{-\lambda\left(\tau_{i}-t\right)}\right)-\left(\tau_{i}-t\right) e^{-\lambda\left(\tau_{i}-t\right)}\right]
$$

The duration measures of the four specific one-factor HJM models analyzed in this study are summarized below.

\subsubsection{Summary of Duration Measures for Certain One-Factor HJM Models $\left(D_{H J M}(t)\right)$}

Constant Volatility $(\sigma(t, T, \omega)=\sigma)$ :

$$
D_{H J M}(t)=\frac{\sum_{i=1}^{n} C_{i}\left(\tau_{i}-t\right) P\left(t, \tau_{i}\right)}{\sum_{i=1}^{n} C_{i} P\left(t, \tau_{i}\right)}
$$

Exponentially Decaying Volatility $\left(\sigma(t, T, \omega)=\sigma e^{-\lambda(T-t)}\right)$ :

$$
D_{H J M}(t)=\frac{\sum_{i=1}^{n} C_{i} P\left(t, \tau_{i}\right)\left(1-e^{-\lambda\left(\tau_{i}-t\right)}\right) / \lambda}{\sum_{i=1}^{n} C_{i} P\left(t, \tau_{i}\right)}
$$

Constant Decay Volatility $(\sigma(t, T, \omega)=\sigma /(1+T-t))$ :

$$
D_{H J M}(t)=\frac{\sum_{i=1}^{n} C_{i} P\left(t, \tau_{i}\right) \ln \left(1+\tau_{i}-t\right)}{\sum_{i=1}^{n} C_{i} P\left(t, \tau_{i}\right)}
$$




$$
\begin{aligned}
& \text { Humped Volatility }\left(\sigma(t, T, \omega)=\sigma(1+\gamma(T-t)) e^{-\lambda(T-t)}\right) \\
& D_{H J M}(t)=\frac{1}{\sum_{i=1}^{n} C_{i} P\left(t, \tau_{i}\right)} \sum_{i=1}^{n} C_{i} P\left(t, \tau_{i}\right) \frac{\gamma}{\lambda}\left[\left(\frac{1}{\gamma}+\frac{1}{\lambda}\right)\left(1-e^{-\lambda\left(\tau_{i}-t\right)}\right)-\left(\tau_{i}-t\right) e^{-\lambda\left(\tau_{i}-t\right)}\right]
\end{aligned}
$$

\subsection{Convexity Measures of One-Factor HJM Models}

Following the same framework with the above analysis, we extend the approach of $\mathrm{Au}$ and Thurston for one-factor HJM models for convexity. The gamma of a coupon bond is derived by taking the second derivative of the bond price with respect to the instantaneous spot rate. Gamma of the bond price under the one-factor HJM models can be represented as follows:

$$
\begin{aligned}
\frac{\partial^{2} B(t, T)}{\partial f(t, t)^{2}} & =-\sum_{i=1}^{n} C_{i} \frac{\partial P\left(t, \tau_{i}\right)}{\partial f(t, t)} \frac{1}{\sigma(t, t, w)^{2}}\left(\int_{t}^{\tau_{l}} \sigma(t, v, \omega) d v\right)^{2} \\
& =\sum_{i=1}^{n} C_{i} P\left(t, \tau_{i}\right) \frac{1}{\sigma(t, t, w)^{2}}\left(\int_{t}^{\tau_{I}} \sigma(t, v, \omega) d v\right)^{2}
\end{aligned}
$$

As it is seen from above equation, the gamma of a coupon bond is a function of the volatility of forward rate, the coupon payments, and the delta of zero coupon bonds maturing at the respective coupon payment dates. The gamma of the coupon bond can be readily mapped onto a continuous time version of convexity by dividing the gamma by the coupon bond price. Thus, under the HJM framework with deterministic volatilities, the convexity of a coupon bond, Conv $_{H J M}$, can be represented as follows ${ }^{25}$ :

$$
\operatorname{Conv}_{H J M}(t)=-\frac{1}{\sum_{i}^{n} C_{i} P\left(t, \tau_{i}\right)} \sum_{i=1}^{n} C_{i} \frac{\partial P\left(t, \tau_{i}\right)}{\partial f(t, t)} \frac{1}{\sigma(t, t, w)} \int_{T}^{\tau_{I}} \sigma(t, v, \omega) d v
$$

\footnotetext{
${ }^{25}$ Fruhwith (2001) follows a similar approach to derive the convexity measures of one-factor HJM models. Our results agree with the results of Fruhwirth (2001).
} 
For constant volatility, $\sigma(t, T, \omega)=\sigma, \operatorname{Conv}_{H J M}(t)$ reduces to the continuous time version of convexity, $\operatorname{Conv}_{C}(t)$, given by equation (7). For an exponentially decaying volatility, $\sigma(t, T, \omega)=\sigma e^{-\lambda(T-t)}$ where $\lambda$ is a positive constant, the gamma of a zero coupon bond derived from equation (23) corresponds to the gamma given by Jarrow and Turnbull (1996, p. 493) for the same volatility function.

We derived the convexity functions of the four one-factor HJM models considered in the study. These measures are summarized in the following subsection.

\subsubsection{Summary of Convexity Measures for Certain One-Factor HJM Models (Conv $\left.{ }_{H J M}(t)\right)$}

Constant Volatility $(\sigma(t, T, \omega)=\sigma)$ :

$$
\operatorname{Conv}_{H J M}(t)=\frac{1}{\sum_{i=1}^{n} C_{i} P\left(t, \tau_{i}\right)} \sum_{i=1}^{n} C_{i} P\left(t, \tau_{i}\right)\left(\tau_{i}-t\right)^{2}
$$

Exponentially Decaying Volatility $\left(\sigma(t, T, \omega)=\sigma e^{-\lambda(T-t)}\right)$ :

$$
\operatorname{Conv}_{H J M}(t)=\frac{1}{\lambda^{2} \sum_{i=1}^{n} C_{i} P\left(t, \tau_{i}\right)} \sum_{i=1}^{n} C_{i} P\left(t, \tau_{i}\right)\left(1-e^{-\lambda\left(\tau_{i}-t\right)}\right)^{2}
$$

Constant Decay Volatility $(\sigma(t, T, \omega)=\sigma /(1+T-t))$ :

$$
\operatorname{Conv}_{H J M}(t)=\frac{1}{\sum_{i=1}^{n} C_{i} P\left(t, \tau_{i}\right)} \sum_{i=1}^{n} C_{i} P\left(t, \tau_{i}\right)\left(\ln \left(1+\tau_{i}-t\right)\right)^{2}
$$

$\underline{\text { Humped Volatility }}\left(\sigma(t, T, \omega)=\sigma(1+\gamma(T-t)) e^{-\lambda(T-t)}\right)$

$$
\operatorname{Conv}_{H J M}(t)=\frac{1}{\sum_{i=1}^{n} C_{i} P\left(t, \tau_{i}\right)} \sum_{i=1}^{n} C_{i} P\left(t, \tau_{i}\right) \frac{\gamma^{2}}{\lambda^{2}}\left[\left(\frac{1}{\gamma}+\frac{1}{\lambda}\right)\left(1-e^{-\lambda\left(\tau_{i}-t\right)}\right)-\left(\tau_{i}-t\right) e^{-\lambda\left(\tau_{i}-t\right)}\right]^{2}
$$




\section{CHAPTER 6}

\section{DATA AND METHODOLOGY}

This study is primarily of a computational nature. The main results are based on Monte-Carlo simulations. Monte-Carlo simulations provide the environment to compare the performances of alternative risk measures and immunization strategies under specific yield curve shapes and volatility structures. The inputs of the simulations are estimated from real data. These inputs are the initial forward rates of different maturities, the volatilities of these forward rates, coupon rates and maturities. Different initial yield curve shapes are considered.

In the following sections, first information on the forwards data is presented. Then the initial yield curves are discussed, followed by the estimation of the forward rate volatilities. Then, the procedure of coupon bond determination is examined. The holding periods, immunization criteria, and immunization portfolios are discussed in the subsequent three sections. Then the discretization of continuous-time HJM models is examined. Random number generation and simulation trials are discussed next. The last section is on the output analysis.

\subsection{Forward Rate Data}

Instantaneous forward rate and zero-coupon yield curve data from January 1947 to February 1991 are obtained from McCulloch and Kwon (1993). In this data set, both zerocoupon yields and forward rates are derived by McCulloch (1975) using a tax adjusted cubic spline method. All forward rates are given as percentages per annum and are on a continuously compounded basis. All data are from the afternoon of the last business day of the month indicated. For each month 56 maturities are available. The data are monthly from 0 to 18 months, then quarterly to 2 years, then semiannually to 3 years, then annually to 35 years, and finally a 5-year jump to 40 years. The cubic spline method is used to smooth the forward 
rates and to interpolate the missing monthly forward rates ${ }^{26}$. These monthly initial forward rates are then used to form the coupon bonds and as inputs of the simulation.

\subsection{Initial Yield Curves}

From January 1947 to February 1991, yield curve charts are formed for each month. These yield curves are categorized into different shapes: Flat, declining, increasing, and humped. One representative yield curve from each of these initial yield curve shapes is selected $^{27}$. The selected yield curves are August 1989 for flat, January 1985 for increasing, May 1981 for decreasing, and October 1986 for humped. These yield curves and the corresponding forward rate curves are shown in Figure 1.

\subsection{Forward Rate Volatilities}

For the selected yield curves, forward rate volatilities are estimated using three-factor principal component analysis (PCA, henceforth). Following Rebonato (1998) ${ }^{28}$ and Amin and $\mathrm{Ng}$ (1997), four years of historical data are used for volatility estimations. The forward rate data are from the aforementioned McCulloch and Kwon (1993) data. In the yield curve literature, the first three factors of the PCA are generally attributed to the level, slope and the curvature of the yield curve. In one-factor models, only the level is taken into consideration. Principal component analysis is proposed by Jarrow (1996), Rebonato (1998), Willmott (1998), and James and Weber $(2000)^{29}$ for the estimation of forward rate volatilities, especially for HJM models. After the volatilities are estimated by the PCA method, the missing volatilities are interpolated. Since the McCulloch and Kwon data do not have forward rates for each month, the volatilities corresponding to missing maturities are interpolated by the cubic spline method ${ }^{30}$.

\footnotetext{
${ }^{26}$ Bliss (1997) compares different curve fitting procedures and finds that the McCulloch (1975) cubic spline procedure and Fama and Bliss (1987) bootstrapping procedure outperforms other methods.

${ }^{27}$ The yield curve selection procedure is explained in detail in Technical Appendix 1.

${ }^{28}$ See pp. 51-74.

${ }^{29}$ See Jarrow (1996), pp. 208-213; Rebonato (1996), pp. 51-74; Willmott (1998), pp. 504-507; James and Weber (2000), pp. 455-485.

${ }^{30}$ Bliss (1997) finds that cubic spline method is better than most of the existing alternatives to smooth and interpolate data.
} 


\section{Figure 1}

\section{Initial Yield Curves and Forward Rate Curves}

This figure shows the initial yield curves considered in the study. Using McCulloch and Kwon (1993) monthly yield curve data from January 1947 to February 1991, all initial yield curves are categorized as increasing, decreasing, flat, humped, and other. A representative yield curve is selected from each group. The selected yield curves are as follows: For a flat yield curve, August 1989; for an increasing yield curve January 1985; for a decreasing yield curve, May 1981; and for a humped yield curve, October 1986. In Figures 1.A to 1.D, these yield curves and the corresponding forward rate curves are presented, respectively.

\section{Figure 1.A}

Flat Yield Curve and Forward Rate Curve on August 1989

Zero Coupon Bond Yield Curve, August 1989

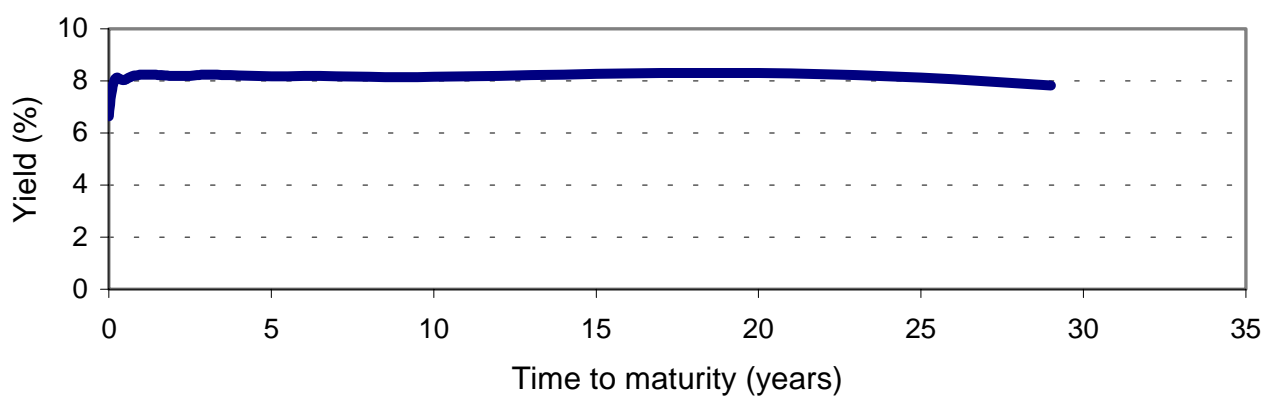

Forward Rate Curve, August 1989

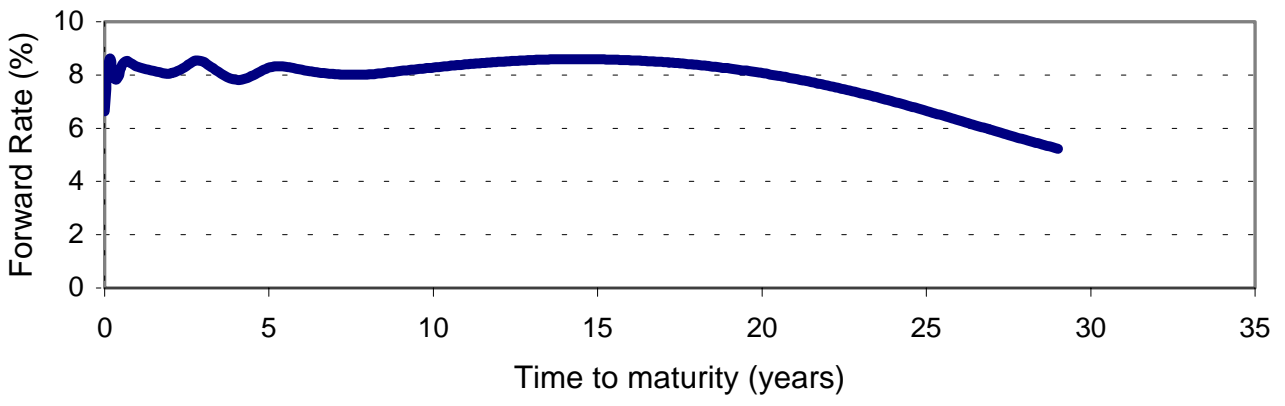




\section{Figure 1.B}

Increasing Yield Curve and Forward Rate Curve on January 1985

Zero Coupon Bond Yield, January 1985

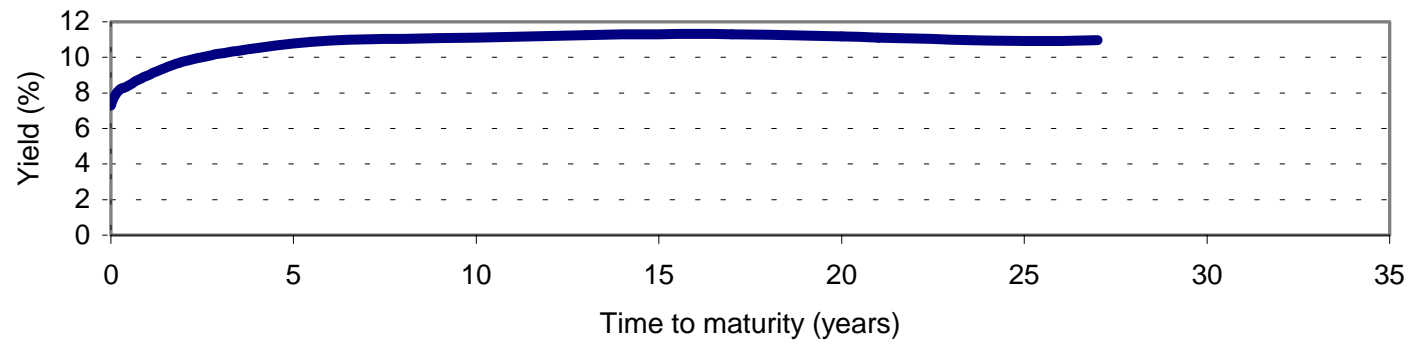

Forward Rate Curve, January 1985

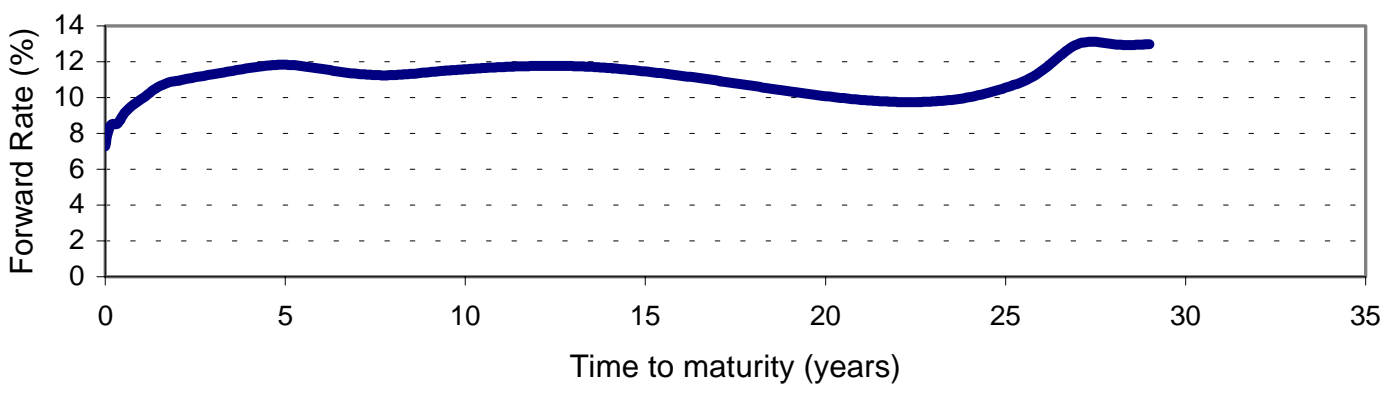


Figure 1.C

Decreasing Yield Curve and Forward Rate Curve on May 1981

Zero Coupon Yield, May 1981

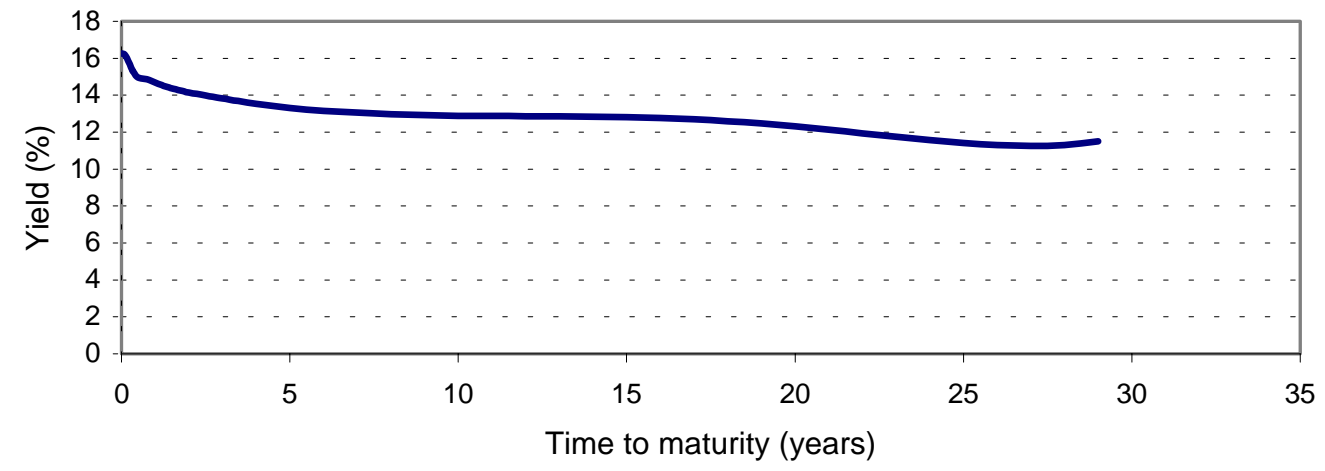

Forward Rate Curve, May 1981

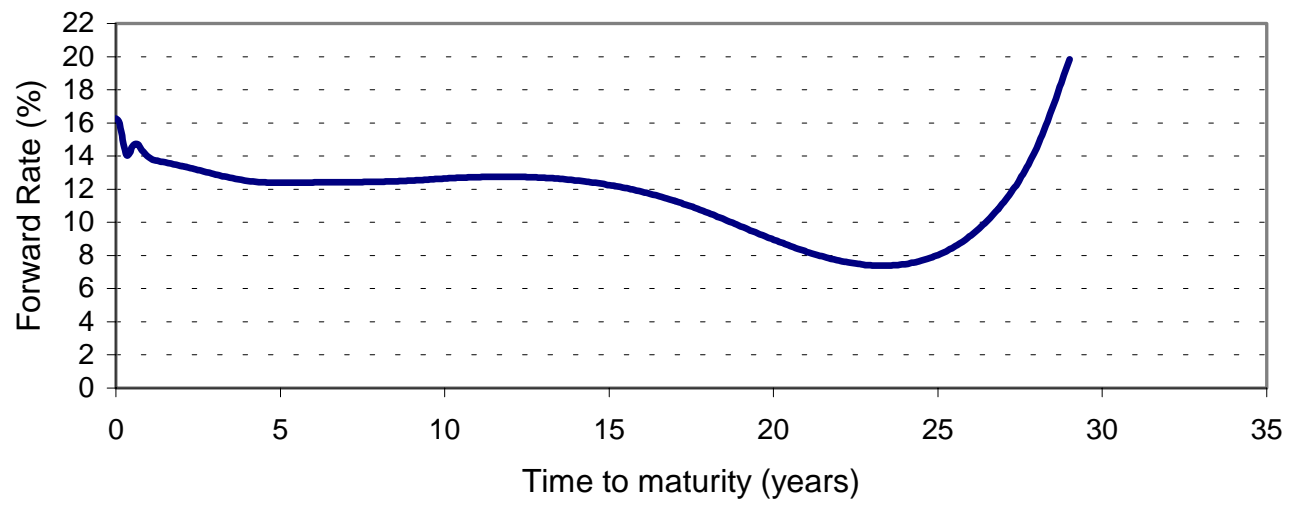


Figure 1.D

Humped Yield Curve and Forward Rate Curve on October 1986

Zero Coupon Yield, October 1986

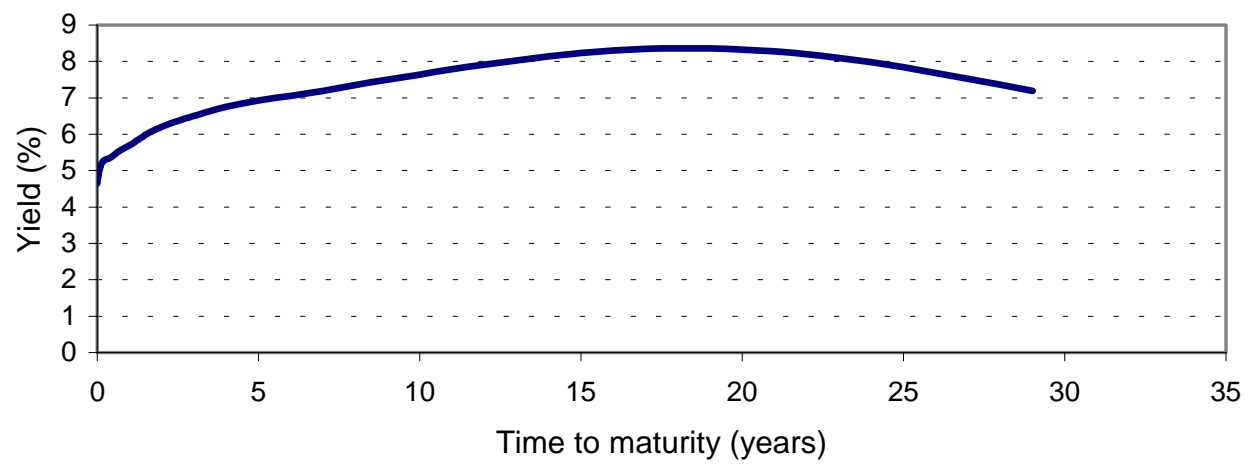

Forward Rate Curve, October 1986

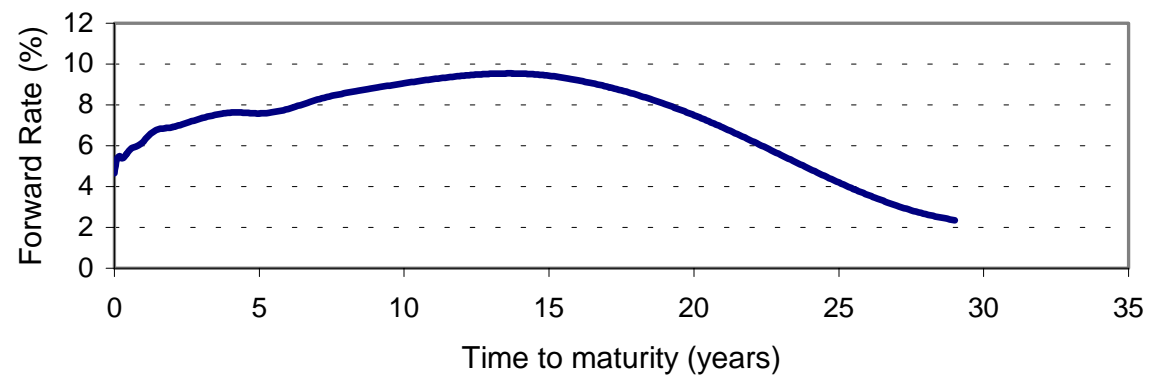


The estimated forward rate volatilities are shown in Figure 2 for the four cases that correspond to flat, increasing, decreasing and humped initial yield curves. The one month forward rate volatilities generally decrease from short term to medium term, but then increase from medium to long term.

The estimated volatilities are fit into the volatility functions of certain one-factor HJM models by regression methods ${ }^{31}$. Four different one-factor HJM models are analyzed for each yield curve. These one-factor models differ in the volatility structure. Following Amin and Morton (1994), only the class of volatilities that depend on time to maturity and constant volatility are considered. In this respect, the most widely used constant volatility (Ho and Lee (1986)), exponential decay volatility (Vasicek (1977)) and constant decay volatility are taken into consideration. Another volatility function considered in this study is the humped volatility proposed by Mercurio and Moraleda $(2000)^{32}$.

To estimate the parameters of the volatility functions, the forward rate volatilities are fit into selected volatility functions by regression methods. For constant, constant decay, and exponential decay volatilities, linear cross-sectional regression is used. For humped volatility, the Marquardt method of non-linear regression is used. The results are presented in Table 1.

As shown in Table 1, Panel A, for the constant volatility function, the expected value of the different maturity forward rate volatilities gives the volatility estimate that will be used in the simulations. The estimated constant volatilities range between $2.08 \%$ to $3.06 \%$, where $2.08 \%$ is observed for decreasing initial yield curve and 3.13\% is observed for increasing initial yield curve.

Table 1, Panel B gives the estimates of the constant decay volatility parameters. For the constant decay volatility, the volatilities of different maturity forward rates on the selected

\footnotetext{
${ }^{31}$ The forward rate volatility estimation procedure for the increasing yield curve case on January 1985 is explained in detail in Technical Appendix 2.

${ }^{32}$ This study can later be extended for other volatility structures that allow HJM to be Markovian The conditions that volatility structures should satisfy for HJM to be Markovian are given by Caverhill (1994), Ritchken and Sankarasubramanian (1995), Jeffrey (1995), and Inui and Kjima (1998). Extending the study for non-Markovian volatility functions may not provide additional insight given the evidence of Amin and Morton (1994) who analyze non-Markovian volatility structures as well as the Markovian ones and find that these non-Markovian volatility structures do not provide additional benefits.
} 
Figure 2

\section{Forward Rate Volatility Curves}

This figure shows the estimated forward rate volatilities. Forward rate volatilities are estimated using three-factor principal component analysis. Four years of historical data are used for volatility estimations. The volatility curve is smoothed and missing volatilities are interpolated by cubic spline method. The forward rate volatilities are the annualized volatilities. Forward rate volatilities are estimated on the selected dates: For a flat yield curve, August 1989; for an increasing yield curve January 1985; for an decreasing yield curve, May 1981; and for a humped yield curve October 1986. Figures 2.A to 2.D give these estimated and smoothed forward rate volatility curves, respectively.

Figure 2.A

Forward Rate Volatility Curve on August 1989

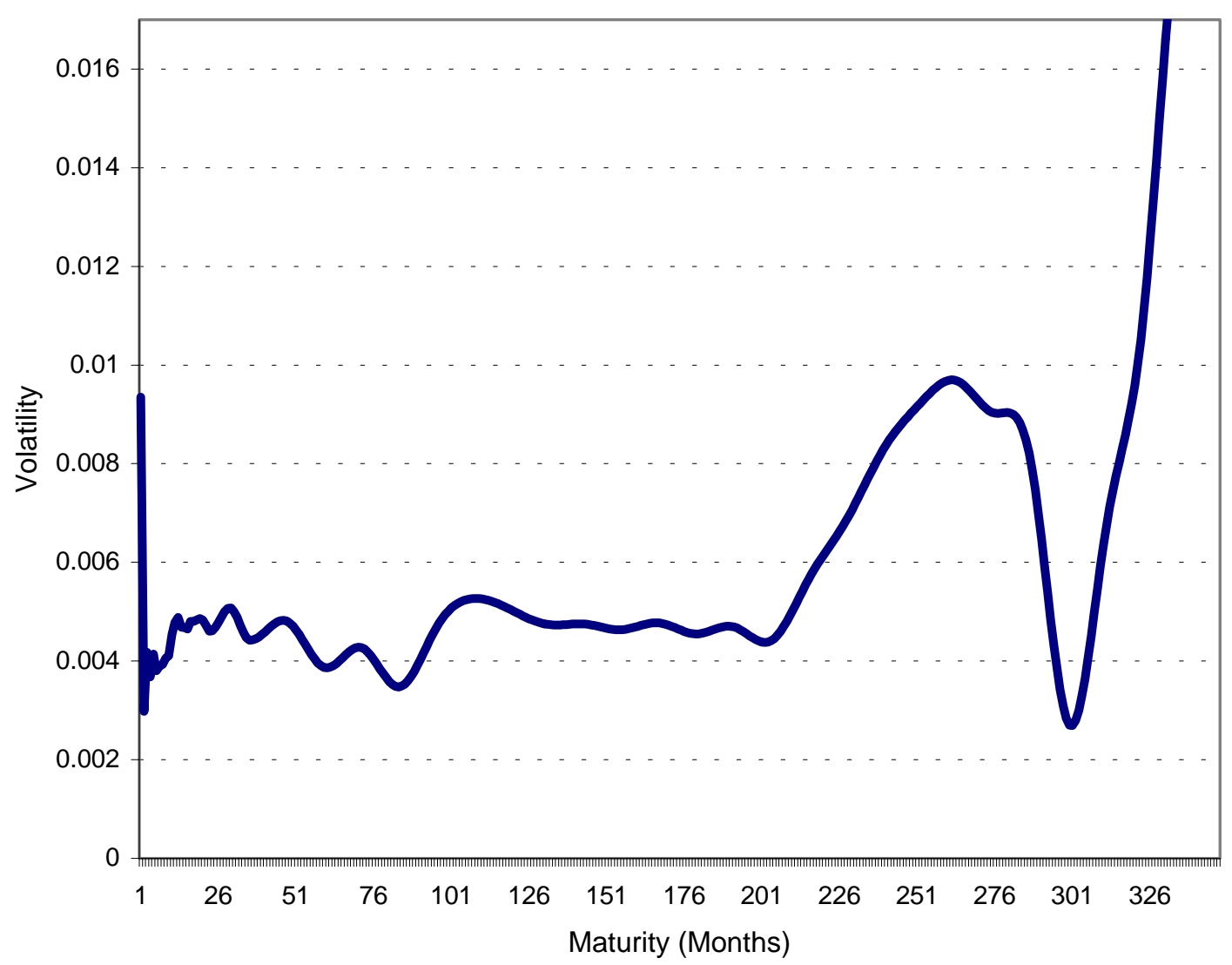


Figure 2.B

Forward Rate Volatility Curve on January 1985

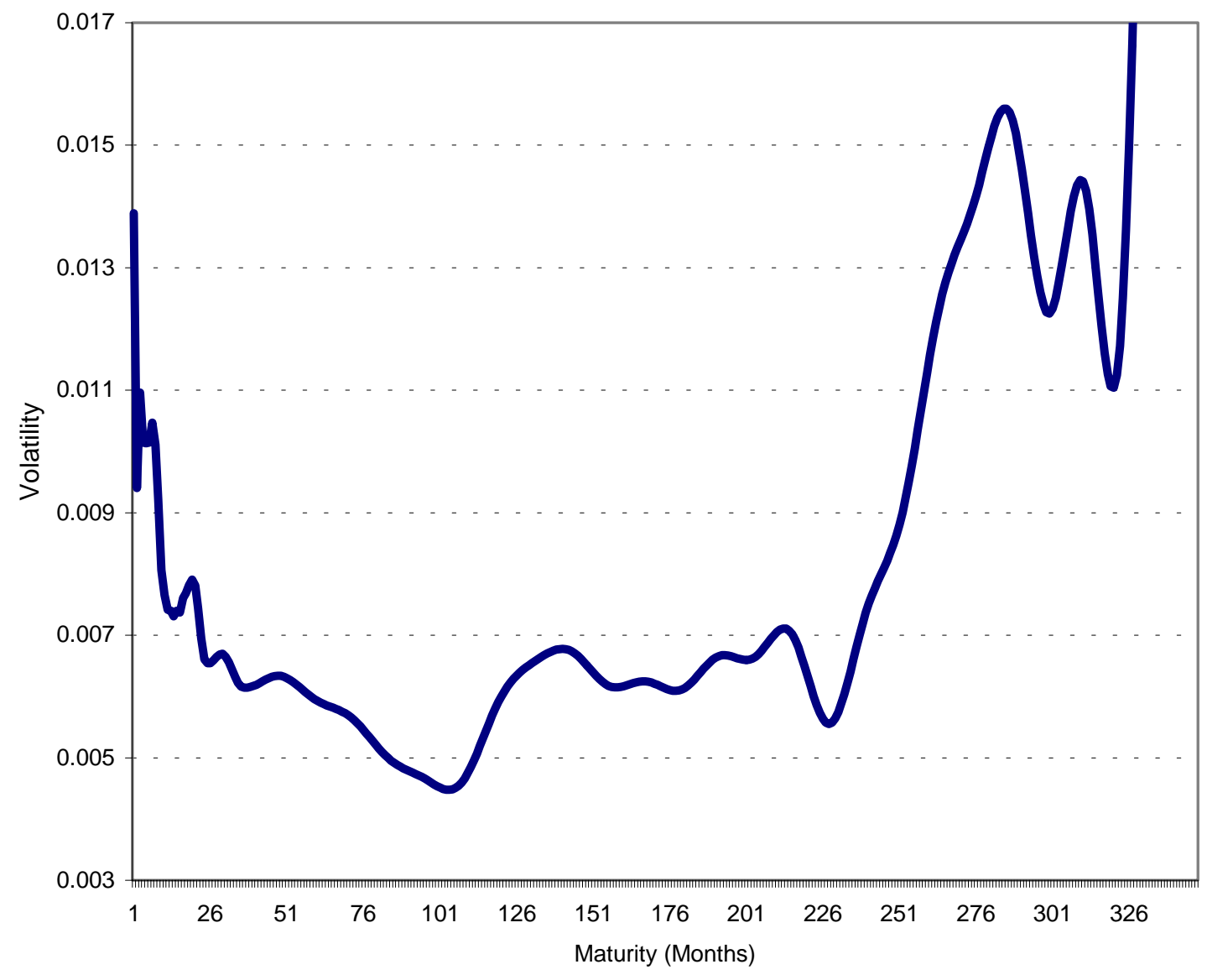


Figure 2.C

Forward Rate Volatility Curve on March 1981

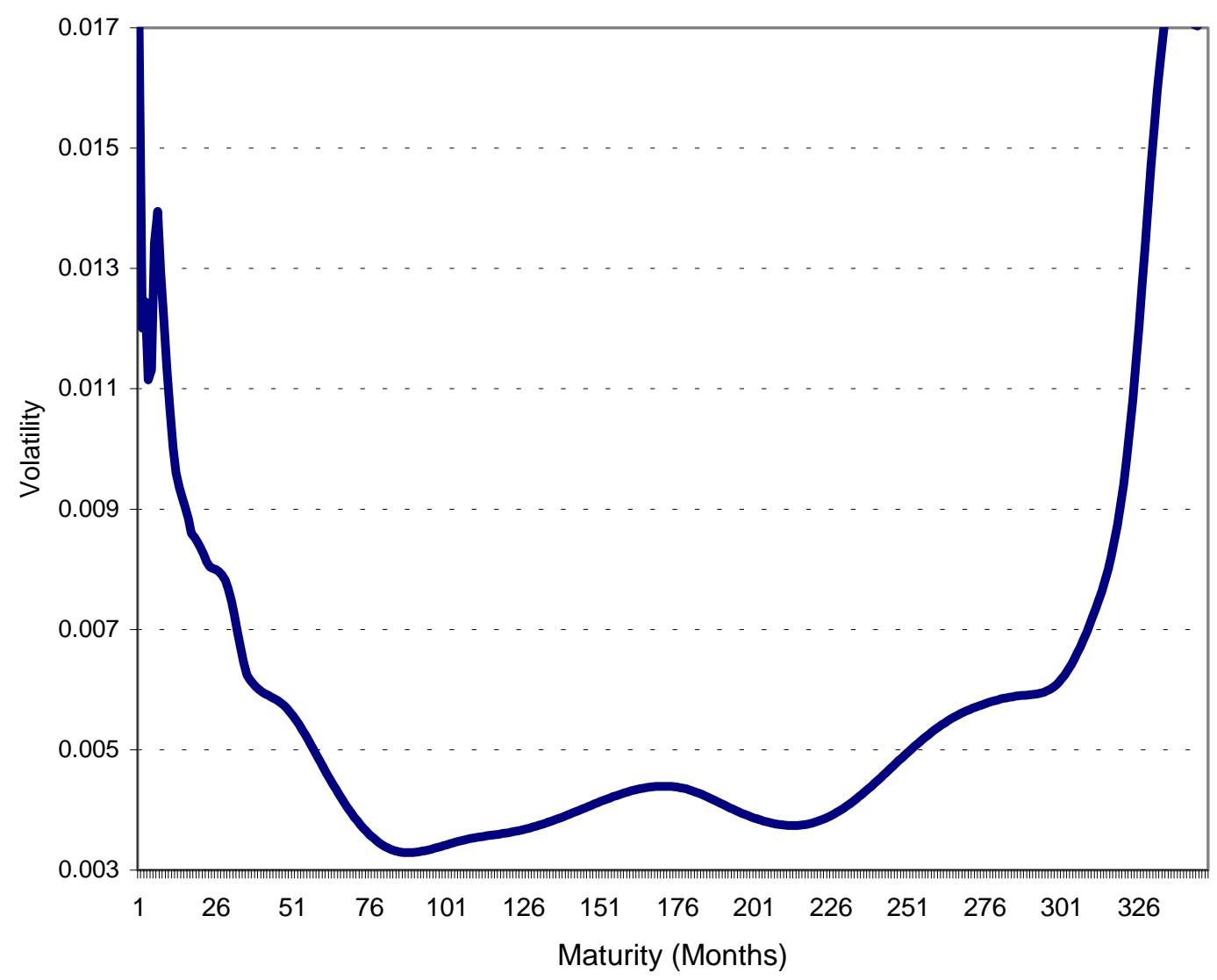


Figure 2.D

Forward Rate Volatility Curve on October 1986

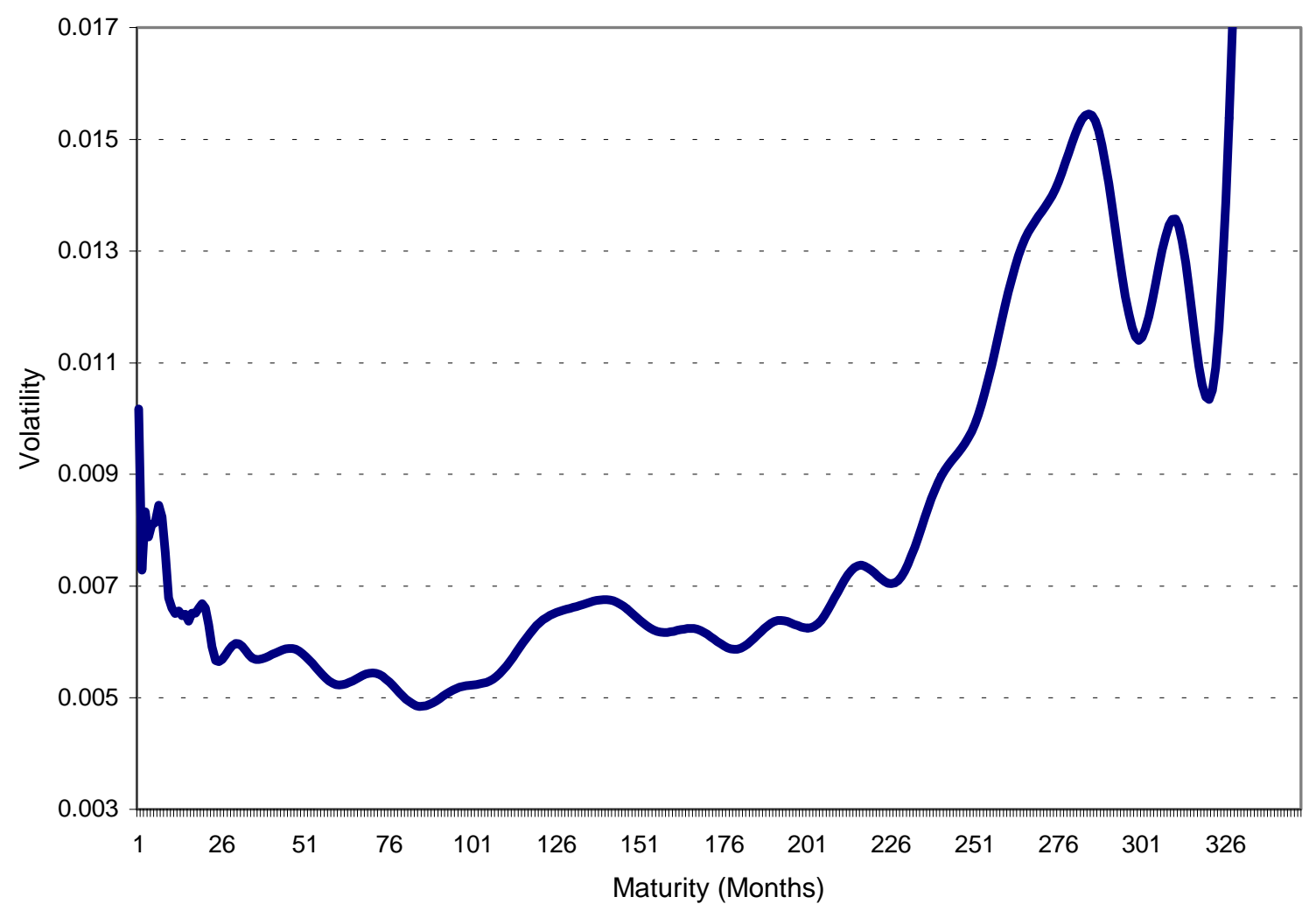




\section{Table 1}

\section{Forward Rate Volatility Functions}

The one-factor HJM model specifies the instantaneous forward rate as follows:

$$
d f(t, T)=\left(\sigma(t, T) \int_{t}^{T} \sigma(t, s) d s\right) d t+\sigma(t, T) d \tilde{W}_{t}
$$

$f(t, T)$ is the instantaneous forward rate at time $t$ for date $T, T>t, W_{t}$ is the Brownian motion at time $t$, and $\sigma(t, T)$ is the stochastic volatility function. Four different volatility functions are considered in Panels A, B, C, and D: Constant, constant decay, exponential decay and humped volatilities. For each volatility function, the parameters are estimated using four years of historical monthly forward rate data of McCulloch and Kwon (1993). Three-factor principal component analysis is used to estimate monthly volatilities. Monthly volatilities are smoothed and missing ones are interpolated by the cubic spline method. These estimated volatilities are fit into the above-mentioned four volatility functions to estimate the parameters. To estimate the parameters of humped volatility, the Marquardt non-linear least squares method is used. For other volatility parameters linear regression method is carried out. This procedure is followed to determine the volatility parameter estimates for August 1989 (flat yield curve), January 1985 (increasing yield curve), May 1981 (decreasing yield curve), and October 1986 (humped yield curve). The volatility estimates presented in the table are the annualized volatilities. In each panel, first the estimates of the volatility parameters are presented. Standard errors are given in parentheses. Then the forward rate volatilities corresponding to one-, five-, and ten-year holding periods are given. To determine these forward rate volatilities, the estimated parameters are used.

\begin{tabular}{|c|c|c|c|c|}
\hline \multicolumn{5}{|c|}{$\begin{array}{c}\text { Panel A: Constant Volatility } \\
\sigma(t, T) \equiv \sigma, \text { a constant } \\
\sigma(t, T)=\hat{\sigma}+\varepsilon\end{array}$} \\
\hline & $\begin{array}{c}\text { Flat } \\
\text { (August 1989) }\end{array}$ & $\begin{array}{c}\text { Increasing } \\
\text { (January 1985) }\end{array}$ & $\begin{array}{l}\text { Decreasing } \\
\text { (May 1981) }\end{array}$ & $\begin{array}{c}\text { Humped } \\
\text { (October 1986) }\end{array}$ \\
\hline$\hat{\sigma}$ & $\begin{array}{c}0.02229 \\
(6.63 \mathrm{E}-04)\end{array}$ & $\begin{array}{c}0.03134 \\
(9.81 \mathrm{E}-04)\end{array}$ & $\begin{array}{c}0.02080 \\
(6.50 \mathrm{E}-04)\end{array}$ & $\begin{array}{c}0.03058 \\
(8.89 \mathrm{E}-04)\end{array}$ \\
\hline \multicolumn{5}{|l|}{$\sigma(0, T)$} \\
\hline $\mathrm{T}=1,5,10$ & 0.02229 & 0.03134 & 0.02080 & 0.03058 \\
\hline
\end{tabular}


Table 1 - Continued

\begin{tabular}{|c|c|c|c|c|}
\hline \multicolumn{5}{|c|}{$\begin{array}{c}\text { Panel B: Constant Decay Volatility } \\
\sigma(t, T) \equiv \sigma /(1+T-t), \sigma \text { a constant. } \\
\quad(1+T-t) \sigma(t, T)=\hat{\sigma}+\varepsilon\end{array}$} \\
\hline & $\begin{array}{c}\text { Flat } \\
\text { (August 1989) }\end{array}$ & $\begin{array}{c}\text { Increasing } \\
\text { (January 1985) }\end{array}$ & $\begin{array}{l}\text { Decreasing } \\
\text { (May 1981) }\end{array}$ & $\begin{array}{c}\text { Humped } \\
\text { (October 1986) }\end{array}$ \\
\hline$\hat{\sigma}$ & $\begin{array}{c}0.40013 \\
(2.38 \mathrm{E}-02)\end{array}$ & $\begin{array}{c}0.57059 \\
(3.49 \mathrm{E}-02)\end{array}$ & $\begin{array}{c}0.33856 \\
(2.12 \mathrm{E}-02)\end{array}$ & $\begin{array}{c}0.55976 \\
(3.23 \mathrm{E}-02)\end{array}$ \\
\hline \multicolumn{5}{|c|}{$\sigma(0, T)$} \\
\hline $\mathrm{T}=1$ & 0.20007 & 0.28530 & 0.16928 & 0.27988 \\
\hline $\mathrm{T}=5$ & 0.06669 & 0.09510 & 0.05643 & 0.09329 \\
\hline $\mathrm{T}=10$ & 0.03638 & 0.05187 & 0.03078 & 0.05089 \\
\hline
\end{tabular}

\begin{tabular}{lcccc}
\hline \multicolumn{5}{c}{ Panel C: Exponential Decay Volatility } \\
\multicolumn{4}{c}{$\sigma(t, T) \equiv \sigma e^{-\lambda(T-t)}, \sigma, \lambda$ constants } \\
& \multicolumn{4}{c}{$\ln (\sigma(t, T))=\ln (\hat{\sigma})-\hat{\lambda}(T-t)+\varepsilon$} \\
\hline Flat & Increasing & Decreasing & Humped \\
& (August 1989) & (January 1985) & (May 1981) & (October 1986) \\
\hline $\ln (\hat{\sigma})$ & -1.04605 & -0.77827 & -0.83711 & -0.83301 \\
& $(3.31 \mathrm{E}-02)$ & $(3.35 \mathrm{E}-02)$ & $(4.52 \mathrm{E}-02)$ & $(2.66 \mathrm{E}-02)$ \\
$\hat{\lambda}$ & -0.03433 & -0.03842 & -0.01433 & -0.04144 \\
$\hat{\sigma}$ & $(1.97 \mathrm{E}-03)$ & $(2.00 \mathrm{E}-03)$ & $(2.69 \mathrm{E}-03)$ & $(1.59 \mathrm{E}-03)$ \\
\hline$\sigma(0, T)$ & 0.01217 & 0.01591 & 0.01500 & 0.01506 \\
$\mathrm{~T}=1$ & & & & \\
$\mathrm{~T}=5$ & 0.01260 & 0.01653 & 0.01522 & 0.01570 \\
$\mathrm{~T}=10$ & 0.01445 & 0.01928 & 0.01611 & 0.01853 \\
\hline
\end{tabular}


Table 1 - Continued

\begin{tabular}{lcccc}
\hline \multicolumn{5}{c}{ Panel D: Humped Volatility } \\
\multicolumn{5}{c}{$\sigma(t, T) \equiv \sigma(1+\gamma(T-t)) e^{-\lambda(T-t)}, \sigma, \lambda$, and $\gamma$ constants. } \\
& $\ln (\sigma(t, T))=\ln (\hat{\sigma})-\hat{\lambda}(T-t)+\ln (1+\hat{\gamma}(T-t))+\varepsilon$ \\
\hline $\ln (\hat{\sigma})$ & Flat & Increasing & Decreasing & Humped \\
& (August 1989) & (January 1985) & (May 1981) & (October 1986) \\
$\hat{\lambda}$ & -0.97802 & -0.76567 & -0.821478 & -0.82332 \\
$\hat{\gamma}$ & $(3.17 \mathrm{E}-02)$ & $(3.38 \mathrm{E}-02)$ & $(4.55 \mathrm{E}-02)$ & $(2.69 \mathrm{E}-02)$ \\
$\hat{\sigma}$ & -0.03110 & -0.03778 & -0.01352 & -0.04094 \\
$\sigma(0, T)$ & $(1.83 \mathrm{E}-03)$ & $(2.02 \mathrm{E}-03)$ & $(2.72 \mathrm{E}-03)$ & $(1.60 \mathrm{E}-03)$ \\
$\mathrm{T}=1$ & 0.000013170 & 0.000006383 & 0.000046051 & 0.000008237 \\
$\mathrm{~T}=5$ & $(0.00000)$ & $(0.00000)$ & $(0.00000)$ & $(0.00000)$ \\
$\mathrm{T}=10$ & 0.013027 & 0.01611 & 0.01523 & 0.01521 \\
\hline
\end{tabular}


dates are multiplied by $(1+\mathrm{T}-\mathrm{t})$. The expected value of these numbers provides us the volatility estimate for the constant decay volatility function. As it can be observed in Figure 1, forward rate volatilities increase significantly from medium to long term. This increase in the forward rate volatilities cannot be captured by the constant decay volatility function since it is restricted to decreasing volatilities. In this respect, the volatilities corresponding to short term rates are estimated to be very high by the constant decay volatility function. As observed from Table 1, Panel B, one-year forward rate volatilities are extremely high, between 17\% and $29 \%$. With respect to the constant decay volatility, forward rate volatilities decrease as time to maturity increases. In this respect, we observe that ten-year forward rate volatilities range from $3.1 \%$ to $5.2 \%$.

The estimated volatility parameter of the exponential decay volatility can be observed in Table 1, Panel C. For exponential decay volatility, first we take the logarithm of different maturity forward rate volatilities on selected dates. Then we regress these numbers on time to maturity. The constant term in the regression provides the estimates of the logarithm of volatility. Thus the exponential of the constant term in the regression gives us the volatility estimate that will be used for the exponential decay volatility. Since the analyzed forward rate volatilities increase considerably from medium to long term ${ }^{33}$, the decay factor of the exponentially decaying volatility is estimated to be negative. According to these parameter estimates, one-year forward rate volatilities are between $1.26 \%$ and $1.65 \%$. Forward rate volatilities increase with time to maturity. Ten-year forward rate volatilities are between $1.71 \%$ and $2.34 \%$. These volatility estimates are well below the ones estimated by the constant decay volatility function. The reason for this difference is that increasing volatility structures cannot be captured by constant decay volatility, whereas they can be captured by exponential decay volatility with a negative decay factor.

Table 1, Panel D shows the parameter estimates of the humped volatility function. For humped volatility, we again take the logarithms of different maturity forward rate volatilities on specific dates and regress these numbers on certain independent variables. The independent variables are time to maturity and a logarithmic function of time to maturity. The constant

\footnotetext{
${ }^{33}$ Refer to Figure 1
} 
term in the equation provides the estimates of the logarithm of the volatility. When we take the exponential of this constant term, we have the volatility estimate for humped volatility function. The Marquardt method of non-linear regression is used to estimate volatility for the humped volatility function. In the humped volatility function there are three parameters. One is a constant volatility parameter, another is an exponential decay factor, and the other is a linear increase factor. The exponential decay factor decreases the volatility as time to maturity increases, but the linear factor increases the volatility as time to maturity increases. In these respects, the interaction of these two factors can lead to increasing, decreasing or humped volatility functions. As can be observed from Table 1, Panel D, the linear factor is always less than $10^{-4}$ and therefore its effect on the volatility is negligible. On the other hand, the exponential decay factor is negative and significant. In these respects, in this study the parameters of the humped volatility function leads to an increasing volatility to capture the forward rate volatility increase observed in the data. The forward rate volatilities of one-, five, and ten-year periods determined by the humped volatility function are very close to those determined by the exponential decay volatility function. Since the linear proportional factor is very small and the decay factor is negative in the humped volatility function, the humped volatility function results in forward rate volatilities that are similar to those found by the exponential decay volatility function.

These volatility functions with the estimated parameters are plotted in Figure 3 against the observed forward rate volatilities given in Figure 2 for each of the four dates considered in the study. As can be observed, the constant decay volatility function provides the worst fit. The best fit is with humped and exponential decay volatilities. The constant volatility case is in between. As expected, the humped and exponential decay volatility functions are very close to each other mainly because the estimated linear factor of the humped volatility is negligible, and the exponential decay factor is the main factor driving the volatility.

To analyze the effects of forward rate volatilities on the immunization performance of different immunization strategies, we categorize forward rates as high and low. We first construct a histogram of the estimated forward rate volatilities. The histogram in given in 
Figure 3

\section{Forward Rate Volatility Functions}

This figure shows the estimated forward rate volatilities and the volatility functions fit to the estimated volatilities. Four volatility functions are considered: Constant, constant decay, exponential decay, and humped volatilities. These volatility functions are as follows: Constant Volatility: $\sigma(t, T) \equiv \sigma$; Constant Decay Volatility: $\sigma(t, T) \equiv \sigma /(1+T-t)$; Exponential Decay Volatility: $\sigma(t, T) \equiv \sigma e^{-\lambda(T-t)} ; \quad$ Humped Volatility: $\sigma(t, T) \equiv \sigma(1+\gamma(T-t)) e^{-\lambda(T-t)}$. In these volatility functions, $\sigma$ is a constant parameter, $\lambda$ is an exponential decay factor, and $\gamma$ is a linear proportional increase factor. These parameters are esteemed using regression methods. The constant, constant decay and exponential decay volatility parameters are estimated by linear regression and the humped volatility parameters are estimated by nonlinear regression. The estimated parameters for each volatility function and each selected date are given in Table 1. The estimated forward rate volatilities and the selected four volatility functions are plotted for the selected dates: For a flat yield curve, August 1989; for an increasing yield curve, January 1985; for a decreasing yield curve, May 1981; and for a humped yield curve, October 1986. Figures 2.A to 2.D give these forward rate volatility curves.

Figure 3.A

Forward Rate Volatility Curves on August 1989

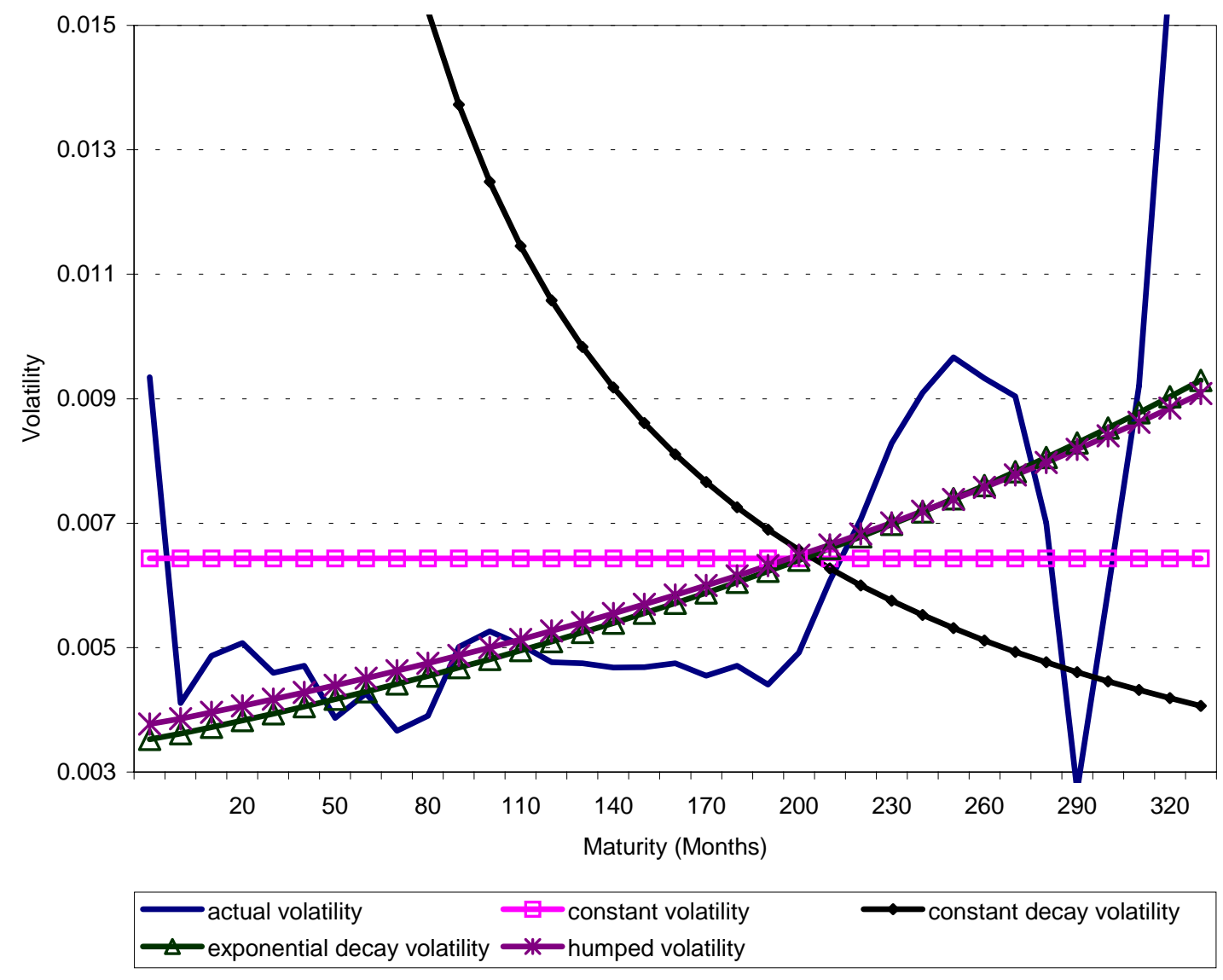


Figure 3.B

Forward Rate Volatility Curves on January 1985

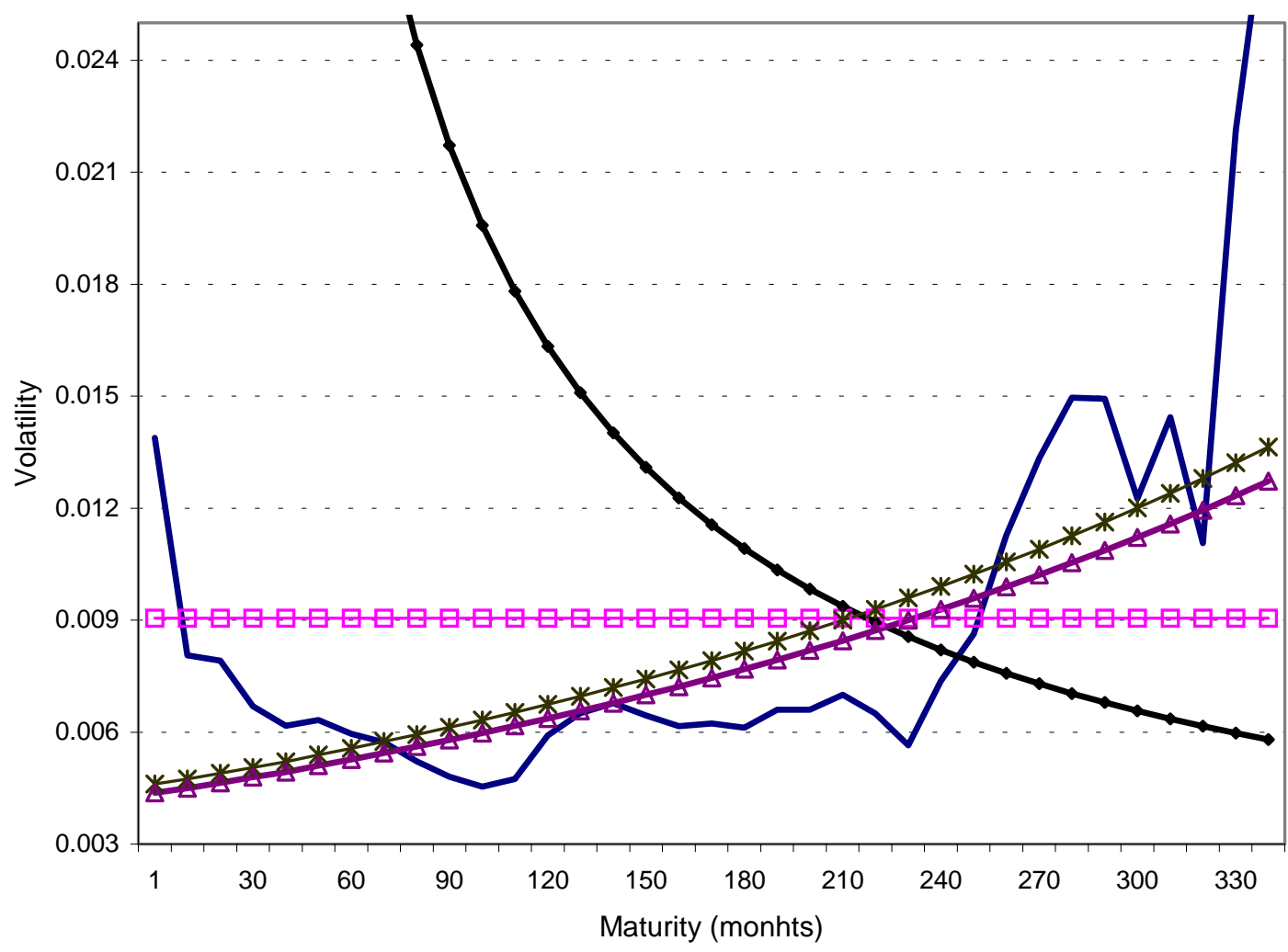

$\begin{array}{ll}\text { actual volatility } & \square \text { constant volatility }\end{array}$ constant decay volatility


Figure 3.C

Forward Rate Volatility Curves on May 1981

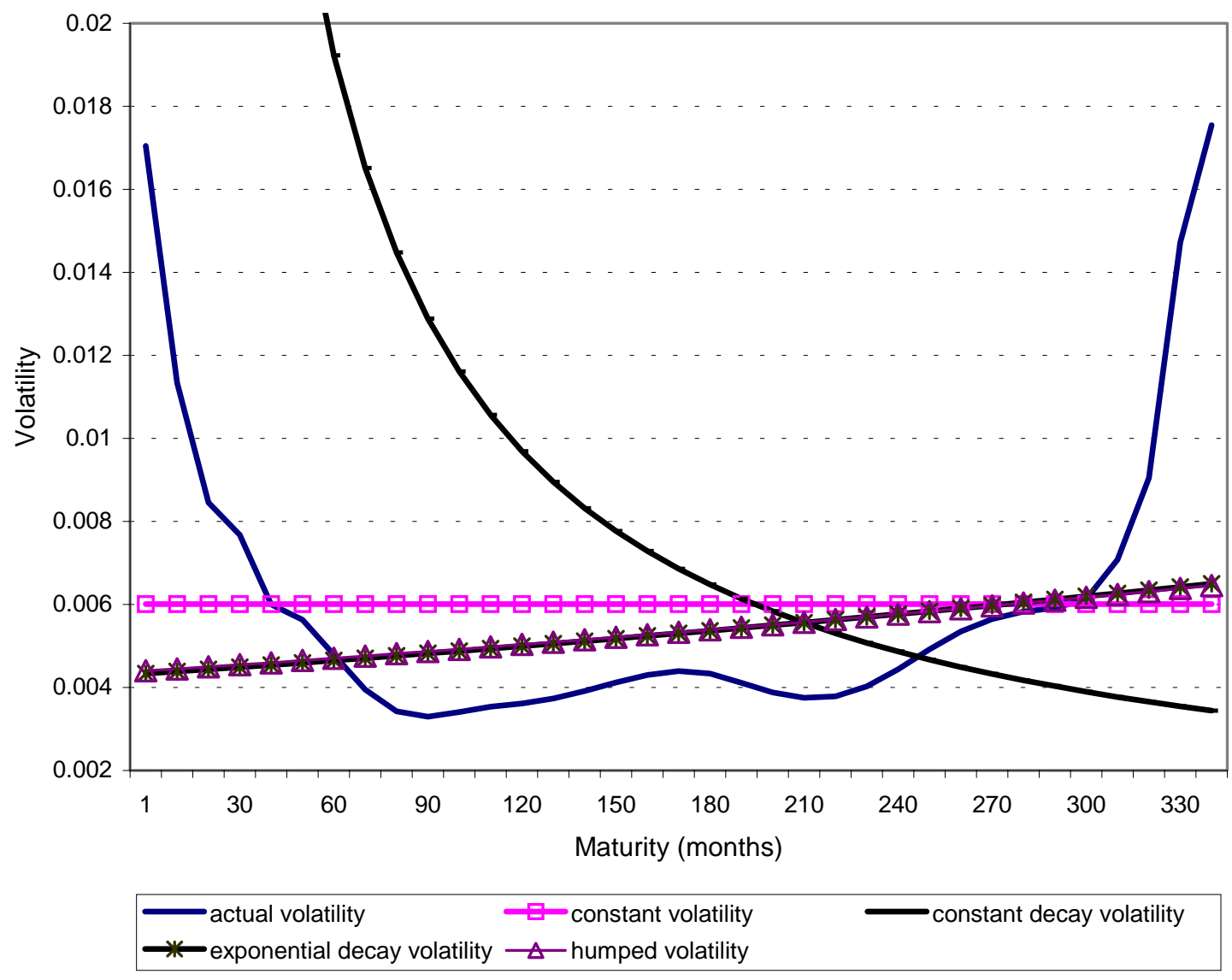


Figure 3.D

Forward Rate Volatility Curve on October 1986

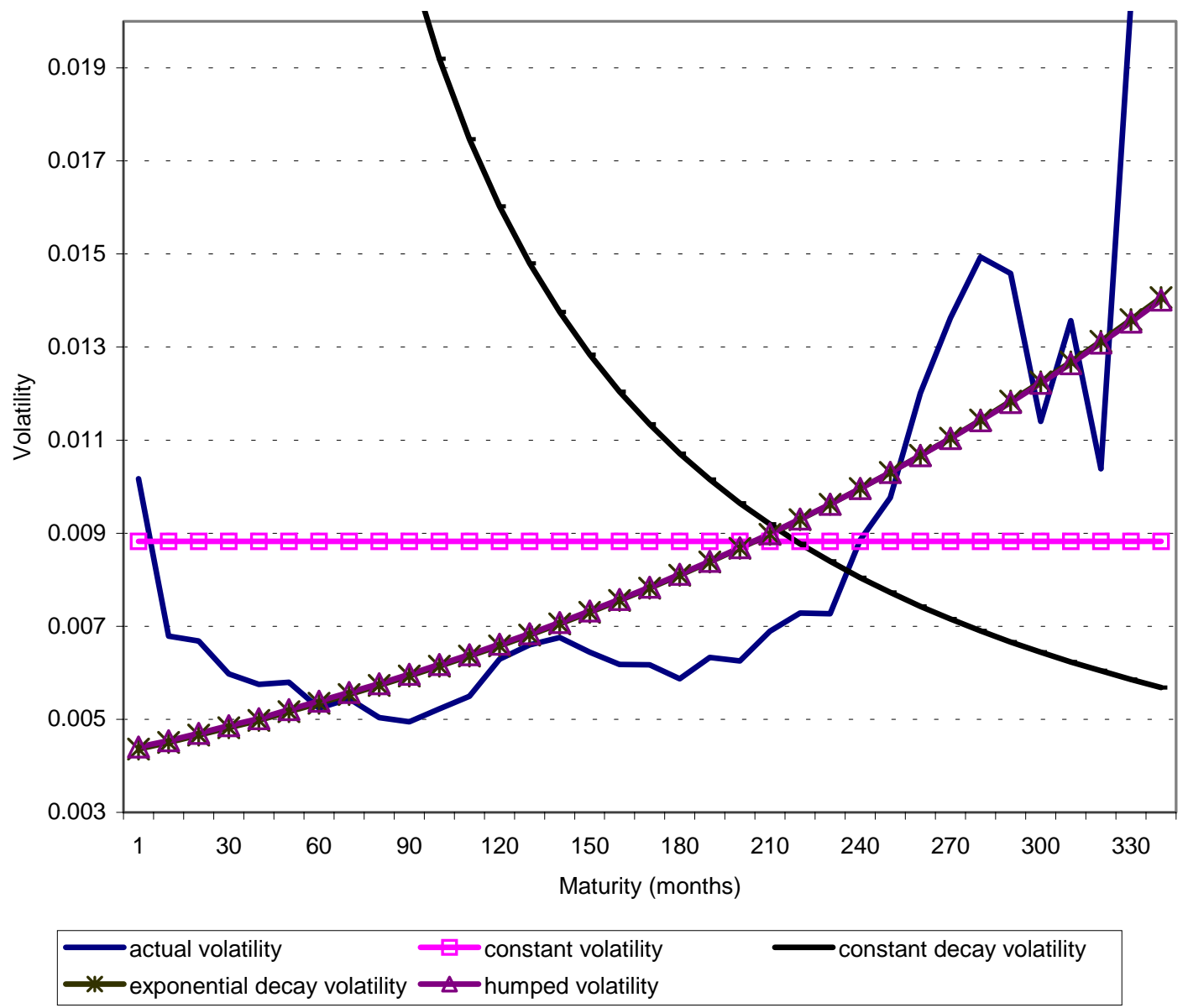


Figure 4 . The mean is $2.22 \%$ and the median is $2.02 \%$. Around $16 \%$ of the forward rate volatilities lie between the median and the mean. This suggests that around $56 \%$ of the forward rate volatilities are below the mean of $2.2 \%$.

To categorize the forward rate volatilities, we use the distributional characteristics of forward rate volatilities from the histogram given in Figure 4 and the fitted volatilities for different holding periods given in Table 1. Since our aim is to analyze the immunization performance, we would like to examine the effects of volatility for each considered HJM model. In this respect, we search for a volatility cutoff point that will enable us to analyze the results for low and high volatility segments of the yield curves under each HJM model considered. We choose $2.25 \%$ as a cut-off point. Any forward rate volatility above this number is considered to be high and any volatility below is considered to be low. According to the forward rate volatility distribution, $67 \%$ of the forward rate volatilities are below $2.25 \%$, and the remaining $33 \%$ are above $2.25 \%$. In this respect, when we mention about low volatility, we cover $67 \%$ of the distribution. Another reason for this cut-off point selection is that when we analyze Table 1, we see that we are able to have at least some high volatility segments in each HJM model considered. In the constant volatility HJM model, the increasing and humped initial yield curves will correspond to high volatilities and the remaining two are low volatility cases. Under constant decay volatility, all are high volatilities due to the misfit of the constant decay volatility to the increasing volatility functions. Under both the exponential decay and humped volatility HJM models, increasing and humped initial yield curves and ten-year holding periods correspond to high volatility cases ${ }^{34}$.

\subsection{Coupon Bonds}

For time zero, the zero coupon bond prices can be derived from the forward rates or the zero coupon bond yields. Table 2, Panel A gives three different maturity zero-coupon bond prices and their yields for the dates that correspond to flat, increasing, decreasing, and humped initial yield curves. The three maturities correspond to the short, medium, and long holding periods, which are one-, five-, and ten-years. Coupon bonds are constructed from the

\footnotetext{
${ }^{34}$ A cutoff point of $2.25 \%$ for volatility makes our results conservative. The results are stronger with any cutoff point higher than $2.25 \%$ volatility
} 


\section{Figure 4}

\section{Histogram of Forward Rate Volatilities}

This figure gives the histogram of the forward rate volatilities estimated from the forward rate data. The forward rate volatilities are estimated by three-factor principal component analysis using four years of historical data. Monthly forward rate data of McCulloch and Kwon (1993) are used. Forward rate volatilities are estimated for four selected dates that correspond to flat, increasing, decreasing and humped initial yield curves. These dates are August 1989, January 1985, May 1981, and October 1986, respectively. The mean is $2.22 \%$, the median is $2.02 \%$, and the standard deviation is $0.93 \%$.

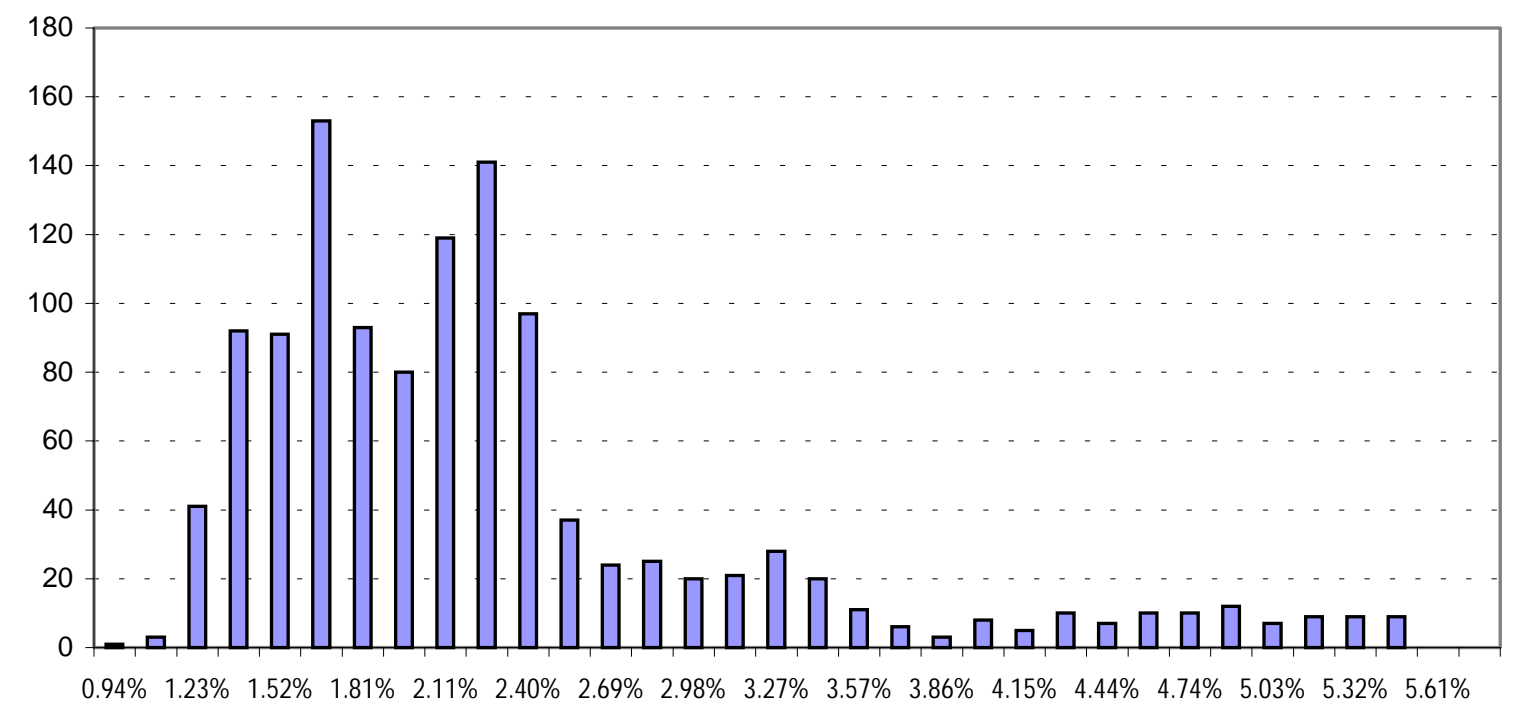


zero coupon bond prices. To determine the coupon rates, CRSP monthly Treasury data is used. For each of the four dates considered, the average of the coupons is computed from the coupon bonds trading on that specific date. These average coupon rates are then used to generate the coupon bonds. The estimated coupon rates for each of the four dates considered are given in Table 2, Panel A. In each of the four different yield curve shapes, all the coupon bonds are assumed to have the same coupon rate ${ }^{35}$. The face value of a coupon bond is assumed to be $\$ 1000$.

\subsection{Holding Periods}

To test the performance of alternative duration measures, one has to decide on the holding period. Holding periods that are considered the most are five and ten years ${ }^{36}$. In these respects, we consider five years as a medium term holding period and ten years as a long-term holding period. To analyze the performances of different risk measures and immunization strategies for short-term holding periods as well, we also consider a one-year holding period.

The target bond is the zero coupon bond that matures at the end of the holding period. In this respect, the yield of the target bond is the target yield by which the performance of the immunization strategies is assessed. Table 2, Panel B shows the prices and the yields of zero coupon bonds for each of the three holding periods and for each of the four initial yield curves.

\subsection{Immunization Criteria}

Different immunization criteria are preferred by different institutions. Some institutions prefer to generate some funds in addition to the sufficient funds generated for liability obligations. These institutions are insurance firms and banks. Pension funds, on the other hand, prefer to have sufficient funds to meet the liability obligations. In these respects,

\footnotetext{
${ }^{35}$ In Fisher and Weil (1971), the coupon rate is fixed as 4\%, whereas in Bierwag, Kaufman, Schweitzer, and Toevs (1981), the coupon rate is fixed as $5 \%$.

${ }^{36}$ Fisher and Weil (1971) use 5, 10, and 20 year holding periods. The holding periods in Cox, Ingersoll, and Ross (1979) are 5, 10, 15, 20, 35, and 50 years. Bierwag, Kaufman, Schweitzer, and Toevs (1981) consider ten-year holding period. Brennan and Schwartz (1983) use five and ten year, Nelson and Schaefer (1983) use five, ten, and fifteen year holding periods. The holding periods of Gultekin and Rogalski (1984) are five and ten years, and the holding period of Bierwag, Kaufman, Latta, and Roberts (1987) is five years.
} 


\section{Table 2}

\section{Coupon Rates, Zero Coupon Bond Prices and Yields}

Four initial yield curve shapes are considered: Flat (August 1989), increasing (January 1985), decreasing (May 1981), and humped (October 1986). For each of these dates, the average of the coupon rates of the coupon bonds trading on that specific date are considered as the coupon rates. CRSP monthly Treasury data are used to determine the coupon rates. The face value of the coupon bonds is taken as \$1000. Zero coupon bond prices are from the McCulloch and Kwon (1993) data. Three different holding periods are considered: Short term (one year), medium term (five years) and long term (ten years). The target bond is the zero coupon bond that has the maturity equal the holding period. The yields of these zero coupon bonds are the target yields that are used to test the immunization strategies. Panel A shows the average coupon rates. Panel B shows the prices and yields of the zero coupon bonds for each of the initial yield curve shapes and holding periods.

\begin{tabular}{lcccc}
\hline \multicolumn{5}{c}{ Panel A: Coupon Rates } \\
\hline Flat & Increasing & Decreasing & Humped \\
\hline Coupon Rate & $9.36 \%$ & $11.38 \%$ & $9.52 \%$ & $10.20 \%$ \\
\hline
\end{tabular}

Panel B: Prices and Yields of Zero Coupon Bonds

\begin{tabular}{lccc}
\hline & 1 Year & 5 Years & 10 Years \\
\hline Price & & & \\
Flat (August 1989) & 921.71 & 665.16 & 442.79 \\
Increasing (January 1985) & 915.15 & 584.53 & 329.59 \\
Decreasing (May 1981) & 862.58 & 513.00 & 275.17 \\
Humped (October 1986) & 945.30 & 708.24 & 466.65 \\
Yield & & & \\
Flat (August 1989) & $8.1523 \%$ & $8.1546 \%$ & $8.1465 \%$ \\
Increasing (January 1985) & $8.8667 \%$ & $10.7388 \%$ & $11.0990 \%$ \\
Decreasing (May 1981) & $14.7832 \%$ & $13.3495 \%$ & $12.9036 \%$ \\
Humped (October 1986) & $5.6248 \%$ & $6.8994 \%$ & $7.6217 \%$ \\
\hline
\end{tabular}


institutions may prefer active or passive portfolio management strategies. Under active portfolio management, if the assets in the immunization portfolio generate funds more than or equal to the liability obligations, the portfolio is successfully immunized. On the other hand, under passive portfolio management, the aim is to generate funds from the asset portfolio as close as possible to the funds needed to meet the liability obligations. Therefore, in passive portfolio management, an asset portfolio that generates funds closest to the liability stream is considered to be successfully immunized.

Fisher and Weil (1971) define a portfolio to be immunized for a holding period if its value at the end of the period, regardless of the course of interest rates during the holding period, is at least as large as it would have been had the term structure been constant throughout the holding period. This definition corresponds to active portfolio management. If an asset portfolio holding period return is greater than or equal to the return on the liabilities, the asset portfolio is immunized. Bierwag, Kaufman, and Toevs (1981) measure immunization performance in several ways. One measure is to compare the distance between the holding period return and the promised yield ${ }^{37}$. Another measure examines the frequency that holding period returns are within five basis points of the target promised yield. Brennan and Schwartz (1983), and Nelson and Schaefer (1983) examine how close the holding period returns of the immunization portfolios are to the target yield. The immunization criteria of these three studies correspond to passive portfolio management.

In these respects, in this study, three different immunization criteria are considered. One of these criteria corresponds to active portfolio management. A portfolio is successfully immunized if the holding period return of the portfolio is greater than or equal to the target yield minus five basis points. In other words, an error of five basis points is allowed. Additionally, we examine the number of portfolios that are within one basis point and one hundred basis points of the target yield. These two measures correspond to a passive portfolio management criterion.

\footnotetext{
${ }^{37}$ The promised yield corresponds to the yield of the target zero-coupon bond. The target zero-coupon bond can be considered as the liability.
} 


\subsection{Portfolio Formation Strategies}

To form immunization portfolios, one has to decide on several criteria. The most important of these criteria are the immunizations strategy, number of securities in the portfolios, the rebalancing period, and the characteristics of the securities in the portfolios.

There are a vast number of immunization criteria that can be considered. In this study, we focus on the two most widely used approaches. These are duration matching, and duration and convexity matching. The duration matching strategy is expected to perform well under small yield curve shifts, whereas the duration and convexity matching strategy is expected to be successful for non-infinitesimal changes in the yield curve as well. The duration of an asset portfolio is matched to the target duration to form duration-matched portfolios. To form duration and convexity matched portfolios, both the duration and the convexity of the asset portfolio are matched to those of the target zero-coupon bond.

The number of securities in an immunization portfolio is also important. In Gultekin and Rogalski (1984) random immunization portfolios from 2 to 10 securities are formed, whereas in Bierwag, Kaufman, Latta, and Roberts (1987) random portfolios consist of 2 to 6 securities. In Brennan and Schwartz (1983), 20 securities constitutes the immunization portfolio. Nevertheless, in most of the studies such as Fisher and Weil (1971), Bierwag, Kaufman and Toevs (1981), and Nelson and Schaefer (1983) only two securities are considered in the immunization portfolio. The number of securities, however, can affect the immunization performance. In this study, two securities are used to form the immunization portfolios with the duration matching strategy, and three securities are used with duration and convexity matching strategy ${ }^{38}$.

After a portfolio is formed, the weights of the securities are changed at every rebalancing period to match the duration, or the duration and convexity of the target bond. Since we use monthly forward rate data, the rebalancing period is one month. The immunization portfolios are self-financing. Only the fractions of securities bought or sold are

\footnotetext{
${ }^{38}$ In future research, the study can then be extended to include varying number of securities to analyze the effect of the number of securities in the immunization portfolios.
} 
determined in each rebalancing period to keep the portfolio self-financing. No transaction costs are assumed.

The characteristics of the securities in the immunization portfolio also affect the immunization performance. In this study, four different types of immunization portfolios are considered depending on the security characteristics of the portfolios: Barbell, bullet, random, and minimum M-Square portfolios. In the following subsections, each of these four portfolios is discussed in detail.

\subsubsection{Barbell Portfolios}

A barbell portfolio consists of bonds with the highest and the lowest durations available. For duration matching, barbell portfolios are formed by one coupon bond that mature at the end of the holding period and another that matures in 20 years. Rolling portfolios are not allowed ${ }^{39}$ and therefore the shortest maturity coupon bond is the one that matures at the end of the holding period. To match both duration and convexity, an additional coupon bond is needed. This additional coupon bond is selected to be a medium term bond. For the one-year holding period, a ten-year bond is selected. For five and ten years, this additional coupon bond has maturity of 12 years and 15 years, respectively.

The same barbell portfolios are used for each HJM model considered in the simulations. This is due to the fact that barbell portfolios are formed by a short maturity bond that matures at the end of holding period and a long bond with a maturity of 20 years. For duration and convexity matching, the third bond is selected to be a medium term bond. In these respects, only the holding period affects the composition of barbell portfolios.

\subsubsection{Bullet Portfolios}

A bullet portfolio consists of bonds that have durations close to the target duration. In this respect, bullet portfolios change as the initial yield curve shape changes since the duration of the coupon bonds will be different at different dates because of different initial forward

\footnotetext{
${ }^{39}$ The portfolio is held up to the end of holding period, and therefore, the maturities of the bonds in the portfolio are greater than or equal to the holding period.
} 
rates. Bullet portfolios change with the benchmark duration as well. Bullet portfolios are formed from bonds that have durations close to the target duration. The target duration is the duration measure that corresponds to the specific one-factor HJM model that is simulated.

For different one-factor HJM models, corresponding duration measures differ. Therefore, the bullet portfolios are different for each HJM model considered in the simulations. Additionally, as the holding period changes, the target duration changes. In this respect, the bullet portfolios of different holding periods will also be different. The bullet portfolios formed for the duration matching strategy consist of two coupon bonds that have the closest durations to the target bond. The bullet portfolios formed for the duration and convexity matching strategy are formed by three coupon-bonds with the closest durations to the target. The bullet portfolios for the duration matching and duration and convexity matching strategies are given in Tables 3 and 4, respectively. The benchmark duration is the duration of the one-factor HJM model that is used in the simulation of the forward rates.

\subsubsection{Random Portfolios}

Gultekin and Rogalski (1984) and Bierwag, Kaufman, Latta, and Roberts (1987) draw random securities to form immunization portfolios. In this study, a total of 100 random portfolios are formed. These portfolios are important to analyze the performance of the immunization strategies. If a bullet or barbell portfolio does not reveal superiority over the random portfolios, then the importance of specific portfolio formation procedures can be questioned.

For the duration matching strategy, the times to maturity of the two coupon bonds in the portfolios are selected randomly. For the duration and convexity matching strategy, an additional coupon bond is needed. The additional bond is selected randomly for each of the 100 random portfolios. Thus we end up having 100 portfolios of three securities for the duration and convexity matching strategy. Three sets of immunization portfolios are formed, 


\section{Table 3}

\section{Bullet Portfolios for Duration Matching Strategy}

This table shows the bullet portfolios that are used as the duration matching portfolios for the 48 cases considered. Four different initial yield curves: Flat (August 1989), increasing (January 1985), decreasing (May 1981), and humped (October 1986), and for each yield curve three holding periods, 1 year, 5 years, and 10 years, are considered. Bullet portfolios consist of two coupon bonds that have the closest duration to the target duration. The target duration depends on the benchmark duration measure. Four different benchmark durations are used. These duration measures are derived for the four different one-factor HJM models considered: constant, constant decay, exponential decay, and humped volatility. Four yield curves, three holding periods, and four volatility functions lead to 48 different cases. The two numbers in each cell are the times to maturity (in months) of the two coupon bonds that form the bullet portfolio.

\begin{tabular}{ccccc}
\hline & $\begin{array}{c}\text { Flat } \\
\text { (August 1989) }\end{array}$ & $\begin{array}{c}\text { Increasing } \\
\text { (January, 1985) }\end{array}$ & $\begin{array}{c}\text { Decreasing } \\
\text { (May, 1981) }\end{array}$ & $\begin{array}{c}\text { Humped } \\
\text { (October, 1986) }\end{array}$ \\
\hline Constant Volatility & & & & \\
1 year & 12,13 & 12,13 & 12,13 & 12,13 \\
5 years & 77,78 & 83,84 & 83,84 & 77,78 \\
10 years & 257,258 & 330,336 & 330,336 & 330,336 \\
Constant Decay Volatility & & & & \\
1 year & 12,13 & 12,13 & 12,13 & 12,13 \\
5 years & 95,99 & 135,146 & 119,141 & 104,109 \\
10 years & 342,348 & 342,348 & 324,330 & 342,348 \\
Exponential Decay Volatility & & & & 12,13 \\
1 year & 12,13 & 12,13 & 12,13 & 77,79 \\
5 years & 78,79 & 83,86 & 83,86 & 222,226 \\
10 years & 219,220 & 341,342 & 336,342 & 12,13 \\
Humped Volatility & & & & 77,79 \\
1 year & 12,13 & 12,13 & 12,13 & 222,235 \\
\hline 5 years & 77,79 & 83,86 & 83,86 &
\end{tabular}




\section{Table 4}

\section{Bullet Portfolios for Duration and Convexity Matching Strategy}

This table shows the bullet portfolios that are used as the duration and convexity matching portfolios for the 48 cases considered. Four different initial yield curves: flat (August 1989), increasing (January 1985), decreasing (May 1981), and humped (October 1986), and for each yield curve three holding periods, 1 year, 5 years, and 10 years, are considered. The bullet portfolios consist of three coupon bonds that have the closest duration to the target duration. The target duration depends on the benchmark duration measure. Four different benchmark durations are used. These duration measures are derived for the four different one-factor HJM models considered: constant, constant decay, exponential decay, and humped volatility. Four yield curves, three holding periods, and four volatility functions lead to 48 different cases. The three numbers in each cell are the times to maturity (in months) of the three coupon bonds that form the bullet portfolio.

\begin{tabular}{ccccc}
\hline & $\begin{array}{c}\text { Flat } \\
\text { (August 1989) }\end{array}$ & $\begin{array}{c}\text { Increasing } \\
\text { (January, 1985) }\end{array}$ & $\begin{array}{c}\text { Decreasing } \\
\text { (May, 1981) }\end{array}$ & $\begin{array}{c}\text { Humped } \\
\text { (October, 1986) }\end{array}$ \\
\hline Constant Volatility & & & & \\
1 year & $12,13,14$ & $12,13,14$ & $12,13,14$ & $12,13,14$ \\
5 years & $77,78,80$ & $83,84,87$ & $83,84,86$ & $77,78,80$ \\
10 years & $257,258,267$ & $330,336,348$ & $330,336,342$ & $270,275,276$ \\
Constant Decay Volatility & & & & \\
1 year & $12,13,14$ & $12,13,14$ & $12,13,14$ & $12,13,14$ \\
5 years & $90,95,99$ & $135,146,157$ & $119,141,158$ & $100,104,109$ \\
10 years & $336,342,348$ & $336,342,348$ & $324,330,336$ & $336,342,348$ \\
Exponential Decay Volatility & & & & \\
1 year & $12,13,14$ & $12,13,14$ & $12,13,14$ & $12,13,14$ \\
5 years & $77,78,79$ & $82,83,86$ & $82,83,86$ & $77,79,80$ \\
10 years & $219,220,224$ & $341,342,347$ & $330,336,342$ & $222,226,235$ \\
Humped Volatility & & & & \\
1 year & $12,13,14$ & $12,13,14$ & $12,13,14$ & $12,13,14$ \\
5 years & $76,77,79$ & $82,83,86$ & $82,83,86$ & $76,77,79$ \\
10 years & $221,225,230$ & $330,336,347$ & $330,336,342$ & $220,222,235$ \\
\hline
\end{tabular}


one for each holding period. For each of the holding periods, the shortest maturity coupon bond is not allowed to have a maturity less than that of the target zero-coupon bond ${ }^{40}$.

\subsubsection{Minimum M-Square Portfolios}

Fong and Vasicek (1983) develop the M-square measure and state that duration matched portfolios that have minimum M-squares should be immunized against both parallel and non-parallel shifts in the yield curve. To test this hypothesis, we form minimum Msquare portfolios with both duration and duration and convexity matching strategies. With each of these strategies, we first form duration-matched portfolios. Among these portfolios, we select the ones with the minimum M-square. With the duration matching strategy, we select two securities, and with the duration and convexity matching strategy, we select three securities. To select these securities, we run optimization programs with MATLAB. The optimization problem is to find the weights of each security in the portfolios that will minimize the M-square such that the duration of the portfolio will be equal to the target duration.

In this study, we use two traditional duration measures and three HJM duration measures. For each of these five duration measures, the minimum M-square portfolios are different, since the weights in the duration-matched portfolios will differ with respect to the duration measure used for immunization. Also, the target duration changes with the holding period. In this respect, for each of the four initial yield curves considered, we run an optimization program for three holding periods and five duration measures. This leads to 60 optimization problems. Since we go through this process for both duration, and duration matching strategies, a total of 120 optimization problems are solved to find the minimum Msquare portfolios.

The minimum M-square portfolios are given in Table 5 and 6, for duration matching and duration and convexity matching strategies, respectively. There are only a few cases where minimum M-square portfolios are also bullet portfolios. These special cases are observed only for one-year holding periods. As observed from Table 5, with the duration

\footnotetext{
${ }^{40}$ The reason for this restriction is to prevent rollover of any coupon bond. In future research, the study can later be extended to allow for rolling over the short maturity coupon bonds.
} 


\section{Table 5}

\section{Minimum M-Square Portfolios for Duration Matching Strategy}

This table shows the times to maturity of the coupon bonds that are in the minimum M-square portfolios formed for the duration matching strategy. Optimization programs are run to find the portfolios with the minimum M-square among all duration matched portfolios. In this respect, for each duration measure, each holding period, and each initial yield curve considered, an optimization is carried out. This leads to 60 optimizations. In each portfolio there are two securities. The duration measures used for duration matching are Macaulay, Fisher-Weil, the HJM exponential decay volatility, the HJM constant decay volatility, and the HJM humped volatility. The four initial yield curves are: Flat (August 1989), increasing (January 1985), decreasing (May 1981), and humped (October 1986). For each yield curve, there are three holding periods, 1 year, 5 years, and 10 years. The two numbers in each cell, are the times to maturity (in months) of the two coupon bonds that form the minimum M-square portfolio.

\begin{tabular}{|c|c|c|c|c|c|}
\hline & Macaulay & Fisher-Weil & $\begin{array}{c}\text { HJM-Exponential } \\
\text { Decay Volatility }\end{array}$ & $\begin{array}{c}\text { HJM- } \\
\text { Constant Decay } \\
\text { Volatility } \\
\end{array}$ & $\begin{array}{c}\text { HJM- } \\
\text { Humped Volatility } \\
\end{array}$ \\
\hline \multicolumn{6}{|l|}{$\begin{array}{l}\text { Flat } \\
\text { (August 1989) }\end{array}$} \\
\hline 1 year & $12,13^{*}$ & $12,13^{*}$ & 27,99 & 13,23 & 52,310 \\
\hline 5 years & 120,302 & 101,171 & 137,216 & 125,127 & 134,200 \\
\hline 10 years & 148,264 & 144,225 & 188,306 & 121,135 & 194,314 \\
\hline \multicolumn{6}{|l|}{$\begin{array}{l}\text { Increasing } \\
\text { (January 1985) }\end{array}$} \\
\hline 1 year & 12,44 & 12,53 & 12,47 & 13,18 & 12,39 \\
\hline 5 years & 111,255 & 98,269 & 77,276 & 107,117 & 117,216 \\
\hline 10 years & 156,309 & 123,285 & 193,269 & 134,155 & 201,336 \\
\hline \multicolumn{6}{|l|}{$\begin{array}{l}\text { Decreasing } \\
\text { (May 1981) }\end{array}$} \\
\hline 1 year & 19,111 & 12,79 & 12,112 & 12,16 & 12,46 \\
\hline 5 years & 80,263 & 85,242 & 78,325 & 73,80 & 66,84 \\
\hline \multicolumn{6}{|l|}{$\begin{array}{l}\text { Humped } \\
\text { (October 1986) }\end{array}$} \\
\hline 1 year & 12,15 & 12,15 & 16,29 & 12,25 & 12,14 \\
\hline 5 years & 100,132 & 71,86 & 81,163 & 60,345 & 79,153 \\
\hline 10 years & 140,268 & 126,276 & 124,166 & 133,136 & 183,279 \\
\hline
\end{tabular}

*Bullet portfolio 


\section{Table 6}

\section{Minimum M-Square Portfolios for Duration and Convexity Matching Strategy}

This table shows the times to maturity of the coupon bonds that are in the minimum M-square portfolios formed for the duration and convexity matching strategy. In each portfolio there are two securities. Optimization programs are run to find the portfolios with the minimum M-square among all duration matched portfolios. In this respect, for each duration measure, each holding period, and each initial yield curve considered, an optimization is carried out. This leads to 60 optimizations. The duration measures used for duration matching are Macaulay, Fisher-Weil, the HJM exponential decay volatility, the HJM constant decay volatility, and the HJM humped volatility duration measures. The four initial yield curves are: flat (August 1989), increasing (January 1985), decreasing (May 1981), and humped (October 1986). For each yield curve, there are three holding periods, 1 year, 5 years, and 10 years. The three numbers in each cell, are the times to maturity (in months) of the three coupon bonds that form the minimum M-square portfolio.

\begin{tabular}{|c|c|c|c|c|c|}
\hline & Macaulay & Fisher-Weil & $\begin{array}{l}\text { HJM-Exponential } \\
\text { Decay Volatility }\end{array}$ & $\begin{array}{c}\text { HJM- } \\
\text { Constant Decay } \\
\text { Volatility }\end{array}$ & $\begin{array}{c}\text { HJM- } \\
\text { Humped Volatility }\end{array}$ \\
\hline \multicolumn{6}{|l|}{$\begin{array}{l}\text { Flat } \\
\text { (August 1989) }\end{array}$} \\
\hline 1 year & $12,13,14^{*}$ & $12,13,14^{*}$ & $12,13,14^{*}$ & $12,13,26$ & $12,13,14^{*}$ \\
\hline 5 years & $60,63,79$ & $60,61,66$ & $60,61,66$ & $60,63,71$ & $60,67,72$ \\
\hline \multicolumn{6}{|l|}{$\begin{array}{l}\text { Increasing } \\
\text { (January 1985) }\end{array}$} \\
\hline 1 year & $12,19,201$ & $12,16,136$ & $12,22,226$ & $12,25,343$ & $12,24,308$ \\
\hline 5 years & $60,64,66$ & $60,64,66$ & $60,66,76$ & $60,66,74$ & $60,66,71$ \\
\hline 10 years & $120,139,288$ & $120,123,236$ & $120,122,162$ & $120,133,134$ & $120,121,155$ \\
\hline \multicolumn{6}{|l|}{$\begin{array}{l}\text { Decreasing } \\
\text { (May 1981) }\end{array}$} \\
\hline 1 year & $12,20,140$ & $12,18,50$ & $12,27,80$ & $12,13,15$ & $12,20,56$ \\
\hline 5 years & $60,64,66$ & $60,64,66$ & $60,66,76$ & $60,66,74$ & $60,66,71$ \\
\hline \multicolumn{6}{|l|}{$\begin{array}{l}\text { Humped } \\
\text { (October 1986) }\end{array}$} \\
\hline 1 year & $12,13,14^{*}$ & $12,13,14^{*}$ & $12,13,14^{*}$ & $12,13,26$ & $12,13,14^{*}$ \\
\hline 5 years & $60,61,66$ & $60,64,84$ & $60,67,72$ & $60,64,69$ & $60,61,66$ \\
\hline 10 years & $120,133,225$ & $120,134,191$ & $120,131,158$ & $120,138,175$ & $120,121,144$ \\
\hline
\end{tabular}

*Bullet portfolio 
matching strategy, only under a flat initial yield curve and for a one-year holding period are the portfolios formed by traditional duration measures of Macaulay and Fisher-Weil bullet and minimum M-square portfolios. Table 6 shows that, with the duration and convexity matching strategy and for a one-year holding period, all portfolios except the ones formed by the HJM constant decay volatility duration measures under flat and increasing initial yield curves are both bullet and minimum M-square portfolios.

These results contradict those of Bierwag, Fooladi and Roberts (1993). According to Bierwag et. al., bullet portfolios are minimum M-square portfolios for a set of bonds with identical coupon rates. In this study, coupon rates are constant and therefore according to Bierwag et al the minimum M-square portfolios should be bullet portfolios, which they are not. Bierwag et al show that a bullet portfolio is a minimum M-square portfolio if and only if the minimum M-square is a strictly convex function of duration. Since in this study minimum M-square portfolios are not necessarily the bullet portfolios, this suggests that the minimum M-square is not necessarily a convex function of duration even for constant coupon rates.

\subsection{Discretization of One-Factor HJM Models}

The initial forward rates and volatility functions of the forward rates are the inputs of the simulation of forward rates for the one-factor HJM models. The forward rate evolution of the one-factor HJM models under the equivalent martingale probability measure satisfies the equation below:

$$
d f(t, T)=\sigma(t, T)\left(\int_{t}^{T} \sigma(t, s) d s\right) d t+\sigma(t, T) d \widetilde{W}_{t}
$$

For simulation purposes, continuous time HJM models should be discretized. An Euler scheme provides a satisfactory discretization method for one-factor HJM models. For multifactor HJM models, more complex alternatives should be considered for higher order weak convergence. One of these alternatives is the Milstein scheme discussed in Kloeden and 
Platen (1992), and James and Weber $(2000)^{41}$. For one-factor HJM models, however, the Euler scheme has satisfactory convergence properties ${ }^{42}$.

For a given discretization of a trading interval $\left[\mathrm{t}_{0}, \mathrm{~T}\right], \mathrm{t}_{0}<\mathrm{t}_{1}<\ldots<\mathrm{t}_{\mathrm{N}}=\mathrm{T}$, the forward rate equation for a one-factor HJM can be discretized as follows by the Euler scheme:

$$
\tilde{f}\left(t_{i+1}, T\right)=\tilde{f}\left(t_{i}, T\right)+\alpha\left(t_{i}, T\right)\left(t_{i+1}-t_{i}\right)+\sigma\left(t_{i}, T\right)\left(\widetilde{W}_{t_{i+1}}-\widetilde{W}_{t_{i}}\right) \quad, \quad i=0,1, \ldots N
$$

where,

$\widetilde{f}\left(t_{i}, T\right)$ : Value of the approximation of $f\left(t_{i}, T\right)$ at the discretization time $\mathrm{t}_{\mathrm{i}}^{43}$.

The order of convergence measures the efficiency of an approximation scheme. The higher the convergence order, the faster the approximation converges. As shown by Duanmu (1994), the weak convergence property of the Euler scheme is satisfactory for one-factor HJM models. In this respect, the Euler scheme will be used for the simulations of this study.

\subsection{Random Variables}

Another important point related to the simulations is the generation of normal random variables. There are several approaches that can be considered. The most widely used are the Box and Muller (1958) method, the polar method of Marsaglia and Bray (1964) and of Atkinson and Pearce (1976), the Kinderman and Ramage (1976) method, and the direct use of the inverse transform method ${ }^{44}$. A detailed discussion of these methods can be found in Law and Kelton $(1991)^{45}$. In this study, the polar method of Marsaglia and Bray is used to generate normal random variates from uniform random numbers. To increase the speed of convergence, antithetic variates are used ${ }^{46}$.

\footnotetext{
${ }^{41}$ See Kloeden and Platen (1992), pp. 345-351, and James and Weber (2000), pp. 362-363.

${ }^{42}$ See Duanmu (1994), pp. 83-89, and James and Weber (2000), pp. 362-363.

${ }^{43}$ In this study, since monthly data are used, the time increment $t_{i+1}-t_{i}$ is taken as one month.

${ }^{44}$ See Odeh and Evans (1974) for FORTRAN implementation of the inverse transformation method.

${ }^{45}$ See pp. 490-492.

${ }^{46}$ Some of the variance reduction techniques are antithetic variates, control variates, importance sampling, and stratified sampling. These variance reduction techniques are discussed in detail in Nelson and Schmeiser (1986), Duffie and Glynn (1995), and Boyle, Broadie, and Glasserman (1997).
} 
The generation of uniform random numbers is the cornerstone of Monte-Carlo simulations. Random number generators can be categorized as linear congruential generators (LCG) and composite generators (CG). LCGs are in the form: $\mathrm{z}_{\mathrm{i}}=\left(\mathrm{az} \mathrm{i}_{\mathrm{i}-1}+\mathrm{c}\right) \bmod (\mathrm{m})$, where $z_{0}$ is the seed and $m$ is the full period ${ }^{47}$.

LCGs have several problems, including differences of compilers in handling the floating point arithmetics and the looping behavior of LCGs. In these respects, CGs are preferred to LCGs. CGs are formed by taking two or more LCGs and combining them in some way. One of the most popular CGs used in simulation is the composite multiple recursive generator of L'Ecuyer (1999). In this study, the random generator of L'Ecuyer will be implemented to generate random numbers. L'Ecuyer (1999) has a full period of approximately $2^{183}$, and is more efficient relative to its similar counterparts.

\subsection{Simulation}

Using the aforementioned Monte-Carlo techniques, forward rate evolutions are simulated over the holding period with each selected one-factor HJM model ${ }^{48}$. Following Boyle, Broadie, and Glasserman (1997), 10,000 simulation trials are conducted. Simulations are run for each of the four one-factor HJM models - constant volatility, exponential decay volatility, constant decay volatility and humped volatility-, the three holding periods -one, five and ten years-, the four initial yield curves - flat, increasing, decreasing, and humped-, and for each of the two immunization strategies used to form portfolios - duration, and duration-convexity matching-. In each scenario, 100 random, one bullet, one barbell, and one minimum M-square portfolios are formed. Under constant volatility HJM model, only two traditional risk measures are used ${ }^{49}$. Under all other three HJM models, two traditional Macaulay and Fisher-Weil-, and one HJM measure, total of three risk measures are used. These lead to 27,192 portfolios (1 immunization strategy*4 initial yield curve*3 holding

\footnotetext{
${ }^{47}$ The looping behavior of LCGs leads $z_{i}$ to take on a value it had previously. In such a case, exactly the same sequence of numbers is generated as before, and this cycle repeats itself. The length of a cycle is called a full period of a generator.

${ }^{48}$ If a simulated forward rate is negative that one is ignored and another forward rate is simulated.

${ }^{49}$ Under the constant volatility HJM model, the HJM duration measure reduces to the Fisher-Weil duration measure.
} 
period*103 portfolios*(3 risk measures*3 HJM model +2 risk measures*1 HJM model $)=27,197$ portfolios).

Under each of the four HJM frameworks, forward rates evolve with respect to the selected HJM model and the HJM risk measures provide the benchmark. The initial forward rate curve is given and the remaining forward rates are simulated. The simulation of forward rates in each period leads to a certain yield curve shift against which we want to hedge. Although it is not likely to give the yield curve shift for each of the simulation cases considered, we provide examples of how forward rates and the initial yield curve changes for August 1989 with a flat initial yield curve under each of the four HJM models considered. These are given in Figure 5.

In each case considered in the simulations, the immunization portfolio's return over the holding period is compared with that of the target bond under the traditional duration and convexity measures as well as the benchmark HJM duration and convexity measures. The standard deviations of the holding period returns are computed as well. The absolute and relative deviations of the holding period returns from the target are examined. The maximum and minimum absolute and relative deviations are determined. Also, the frequency of portfolios that are successfully immunized according to the active portfolio management criterion, as well as the frequency of portfolios that have returns within one basis and 100 basis point of the target are analyzed.

These simulation results reveal the conditions under which HJM risk measures and traditional risk measures differ in terms of yield curve shape, volatility structure, immunization strategy, and holding period. According to the results, the conditions where complex risk measures can be beneficial and where they are not given the implementation costs should be clear. 


\section{Figure 5}

\section{Yield Curve Shifts}

The figure below shows an example of the shift in yield curves in the simulation. The shift in the yield curve is shown for the flat initial yield curve observed on August 1989. Under four HJM frameworks, the initial yield curve and the yield curve after the yield curve shift are plotted. Figure 4.A to 4.D show the yield curve shifts under constant, exponential decay, constant decay, and humped volatility HJM models, respectively. In the figure $\mathrm{y}_{0}$ corresponds to the initial yield curve and $\mathrm{y}_{1}$ corresponds to the yield curve after the shift.

Figure 5.A.

Yield Curve Shift under Constant HJM Framework, August 1989

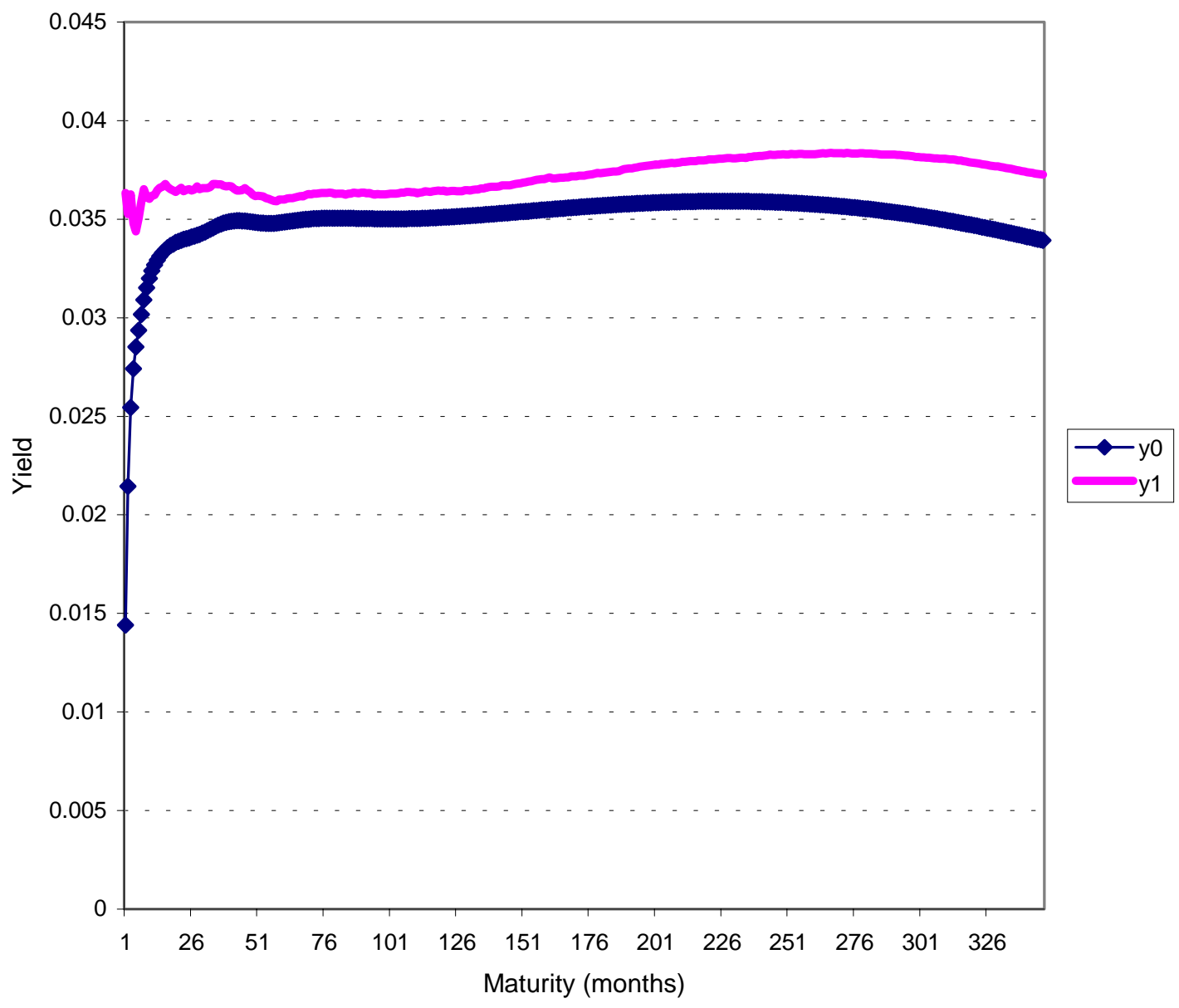


Figure 5.B.

Yield Curve Shift under Exponential Decay Volatility HJM

Framework, August 1989

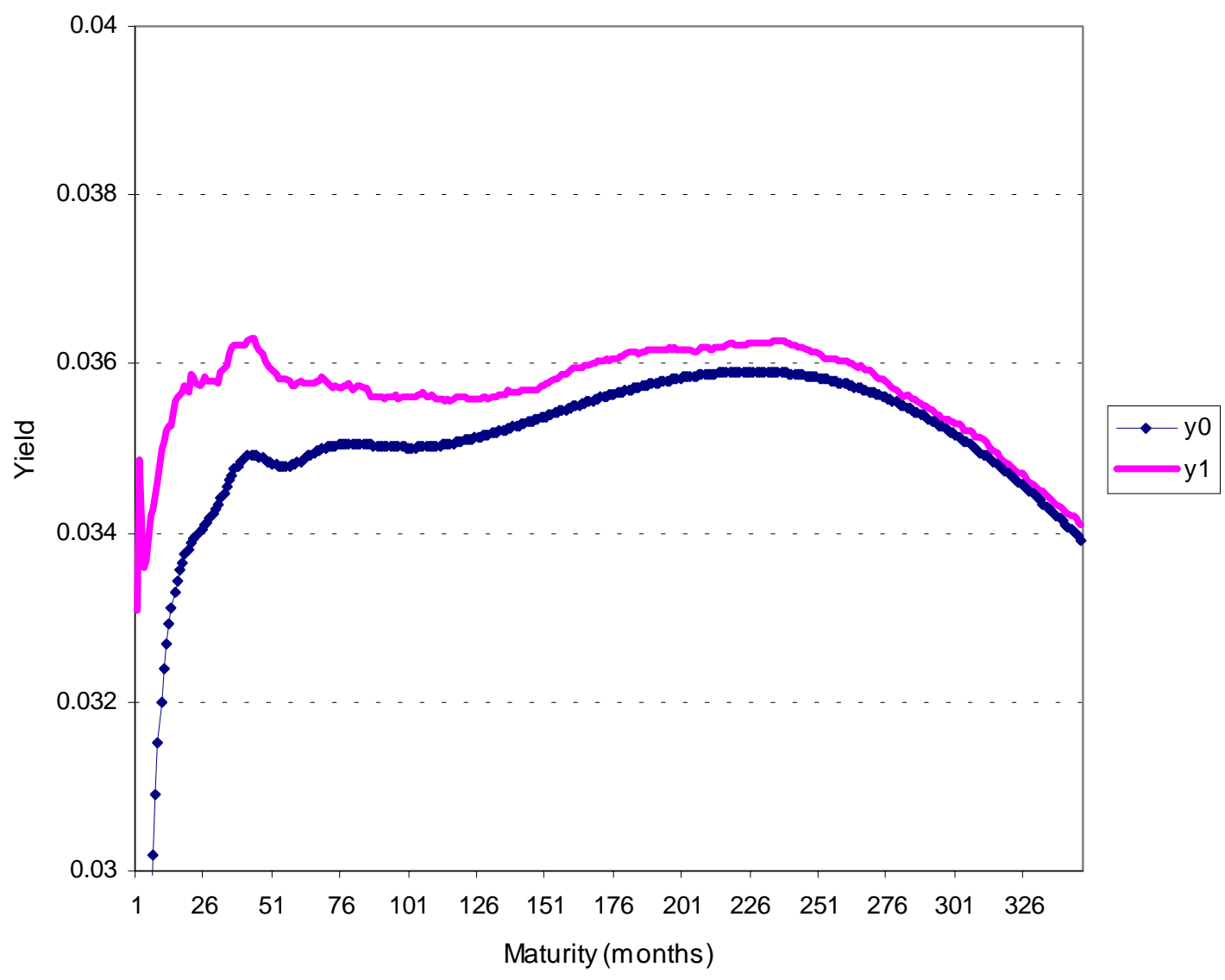


Figure 5.C.

Yield Curve Shift under Constant Decay Volatility HJM Framework, August 1989

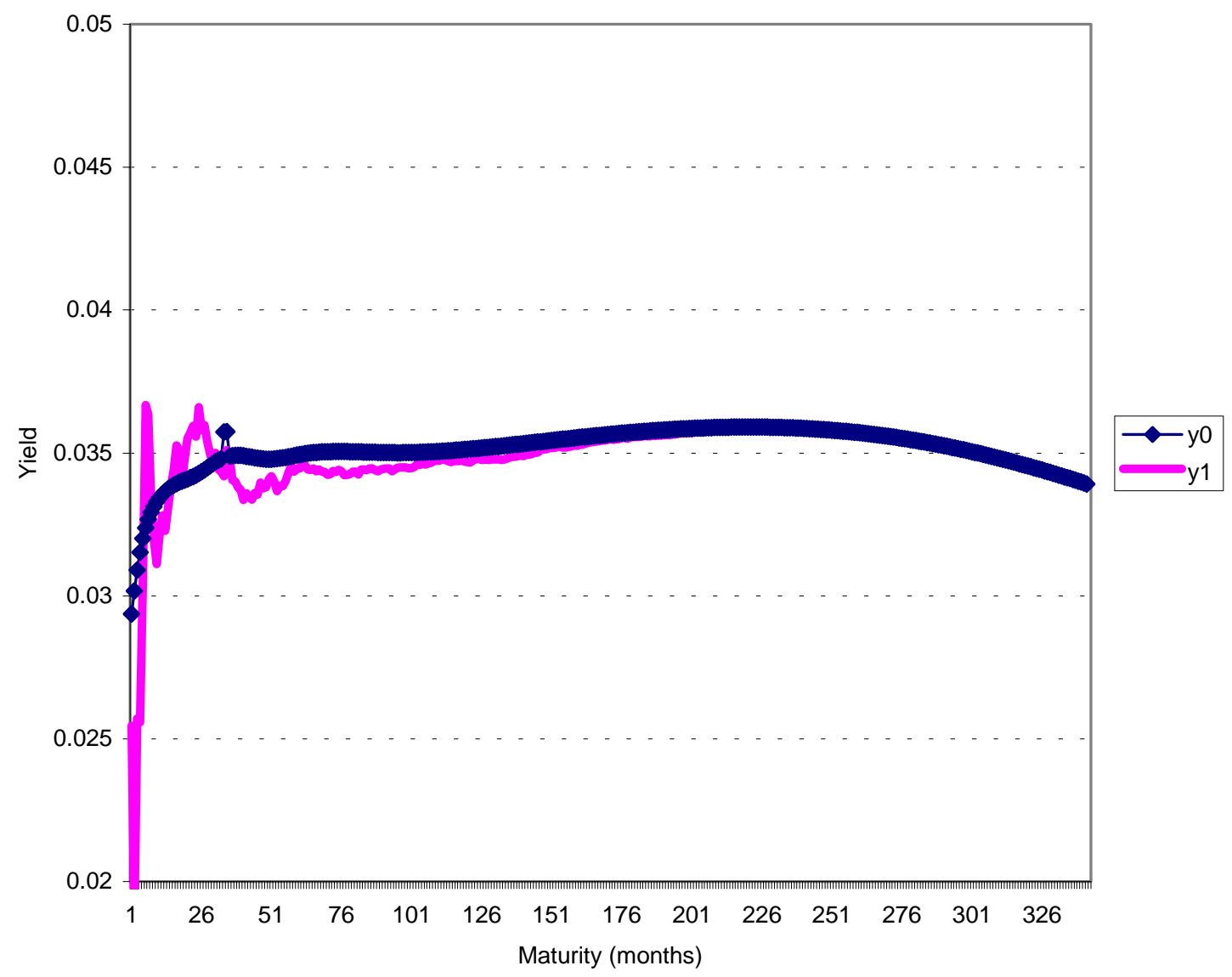


Figure 5.D.

Yield Curve Shift under Humped Volatility HJM Framework, August 1989

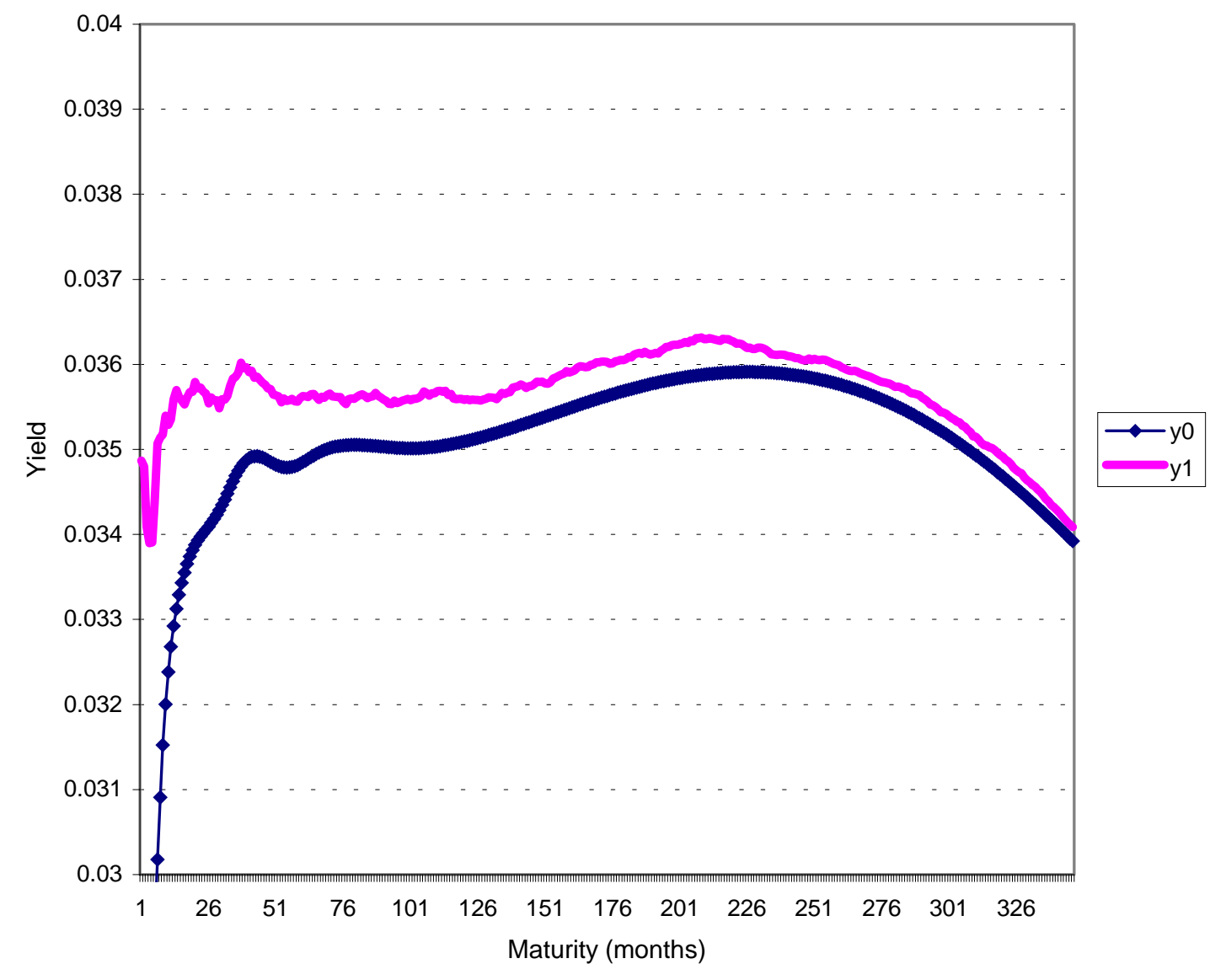




\section{CHAPTER 7}

\section{PERFORMANCE OF DURATION-MATCHED PORTFOLIOS}

Four one-factor HJM models are simulated for four different yield curves and three different holding periods. This results in 48 different scenarios. For each of these scenarios, 102 portfolios are formed. Two of these are bullet and barbell, and the remaining 100 are random portfolios. Each of these portfolios is duration matched with the target zero coupon bond. Three criteria are used to asses the immunization performances. According to the first criterion, an immunization portfolio is successful if the holding period return of the portfolio is greater than or equal to the yield of the target zero coupon bond minus five basis points. This criterion corresponds to active portfolio management. According to the other two criteria, portfolios are successfully immunized if their holding period returns are within one and 100 basis points of the target yield, respectively. These two criteria correspond to passive portfolio management. Two different duration measures are used to match the durations of the portfolios in the constant volatility $\mathrm{HJM}_{\text {model }}{ }^{50}$. For the remaining three HJM models, three duration measures are used. Two of the duration measures are the traditional ones: Macaulay's duration and Fisher-Weil duration. The remaining one is the duration measure derived from the specific one-factor HJM model considered. The factors that affect the immunization performance of different strategies are mainly the forward rate volatilities, holding periods, and portfolio formation strategies. To analyze the effects of these factors, we categorize holding periods as short-, medium-, and long-term, and portfolio formation strategies as random, bullet and barbell. Similarly, to analyze the effects of the volatility, we categorize forward rate volatilities as low and high volatility.

Since the forward rates are simulated according to the HJM models, the HJM duration measures provide the benchmark. In this respect, the analysis can be divided into four parts,

\footnotetext{
${ }^{50}$ In the constant volatility one-factor HJM model, HJM duration measure reduces to the Fisher-Weil duration measure.
} 
for the four different HJM models considered. In the following subsections, the performances of duration matched portfolios are analyzed with respect to the underlying HJM model.

\subsection{Constant Volatility One-Factor HJM Framework}

Table 7 gives the results on the performance of the duration-matched portfolios under the constant volatility HJM framework. Panels A and B present the results for the portfolios matched with Macaulay’s duration and Fisher-Weil duration, respectively.

Table 7, Panel A shows that under an active portfolio management, Macaulay's duration is very successful for short and medium holding periods. Its performance deteriorates considerably for long holding periods when initial yield curves are increasing and humped. As can be observed from Table 1, when we use $2.25 \%$ cutoff point for high and low volatility, the constant forward volatilities of 3.13\% and 3.06\% under increasing and humped yield curves, respectively, correspond to the high forward rate volatility cases. In this respect, Macaulay's duration performs well for short to medium holding periods irrespective of the volatility. Its performance, however, deteriorates significantly in the long run when the forward rate volatilities are relatively high. For short to medium holding periods, more than $96 \%$ of the portfolios are successfully immunized. For ten-year holding period however, only $49 \%$ of the portfolios are successfully immunized under increasing initial yield curve and only $64 \%$ are successfully immunized under humped initial yield curve. Under passive portfolio management, on the other hand, more than $96 \%$ of the portfolios are within 100 basis points of the target yield but generally a range of $5 \%$ to $18 \%$ of the portfolios are within 1 basis point of the target yield ${ }^{51}$.

When the initial yield curve is flat and there is a parallel shift in the yield curve, we expect Macaulay's duration to provide successful immunization. In Table 7 Panel A, we observe this outcome under the flat initial yield curve. When initial yield curve is not flat, however, Macaulay's duration performs poorly for long holding periods. There may be several

\footnotetext{
${ }^{51}$ Only under decreasing yield curve and ten-year holding period $29 \%$ of the portfolios are within 1 basis point of the target yield.
} 


\section{Table 7}

\section{Performance of Duration Matched Portfolios under Constant Volatility HJM Framework}

The performances of 102 portfolios are summarized. Two of the portfolios are bullet and barbell, and the rest are random portfolios. The benchmark portfolios are the ones that are matched with the Fisher-Weil duration. Under constant volatility, the duration measure of the one factor HJM model is the same as the Fisher-Weil duration. The other duration measure is the Macaulay's duration. The results are analyzed according to the initial yield curves and holding periods. Four initial yield curves are considered: Flat (August 1989); increasing (January 1985); decreasing (May 1981); and humped (October 1986). The three holding periods are one-, five-, and ten-years. Panel A presents the results on the performances of immunization portfolios matched by Macaulay's duration. Panel B gives the results on the performances of immunization portfolios matched by Fisher-Weil duration. Three criteria are used to assess the immunization performances of the portfolios. According to one criterion, a portfolio is considered successful in immunization if the holding period return of the portfolio is greater than or equal to the yield of the target zero coupon bond minus 5 basis points. In Panels A and B, "\% Successful" ("\%Unsuccessful") gives the percentage of the portfolios that are successful (unsuccessful) in immunization according to the first criterion. The other two criteria assess the immunization performances by measuring the percentage of portfolios with returns within one and 100 basis points of the target yield. These are given in the next two rows. The maximum and minimum absolute (MaxAD and MinAD) and relative (MaxRD and MinRD) deviations from the target return are presented next. For each deviation, the returns of these portfolios, and the time to maturity of the bonds in the portfolios are given. Standard errors of the returns are in parentheses. Times to maturity are given in months, (T1,T2).

\begin{tabular}{|c|c|c|c|c|c|c|c|c|c|c|c|c|}
\hline \multicolumn{13}{|c|}{ Panel A: Macaulay's Duration } \\
\hline & \multicolumn{3}{|c|}{$\begin{array}{c}\text { Flat } \\
\text { (August, 1989) }\end{array}$} & \multicolumn{3}{|c|}{$\begin{array}{c}\text { Increasing } \\
\text { (January, 1985) }\end{array}$} & \multicolumn{3}{|c|}{$\begin{array}{l}\text { Decreasing } \\
(\text { May, 1981) }\end{array}$} & \multicolumn{3}{|c|}{$\begin{array}{c}\text { Humped } \\
\text { (October, 1986) }\end{array}$} \\
\hline & 1 year & 5 year & 10 year & 1 year & 5 year & 10 year & 1 year & 5 year & 10 year & 1 year & 5 year & 10 year \\
\hline$\%$ Successful & $98.04 \%$ & $98.04 \%$ & $95.10 \%$ & $97.06 \%$ & $97.06 \%$ & $50 \%$ & $97.06 \%$ & $98.04 \%$ & $94.12 \%$ & $98.04 \%$ & $97.06 \%$ & $62.75 \%$ \\
\hline Within 100 basis points & $99.02 \%$ & $99.02 \%$ & $100 \%$ & $96.08 \%$ & $97.06 \%$ & $99.02 \%$ & $97.06 \%$ & $100 \%$ & $98.04 \%$ & $98.04 \%$ & $99.02 \%$ & $100 \%$ \\
\hline Within 1 basis point & $5.88 \%{ }^{* * *}$ & $13.73 \%^{*}$ & $8.83 \%$ & $4.90 \%{ }^{* * *}$ & $11.76 \%$ & $6.86 \%$ & $4.90 \%$ & $17.65 \%$ & $29.41 \%$ & $4.90 \%$ & $9.80 \%$ & $5.88 \%$ \\
\hline $\operatorname{Max} A D$ & $1.14 \mathrm{E}-02$ & $1.00 \mathrm{E}-02$ & $1.50 \mathrm{E}-03$ & $2.58 \mathrm{E}-02$ & $7.27 \mathrm{E}-02$ & $1.60 \mathrm{E}-02$ & 3.79E-02 & 7.60E-03 & $1.18 \mathrm{E}-02$ & $2.09 \mathrm{E}-02$ & $1.04 \mathrm{E}-02$ & 4.72E-03 \\
\hline MaxRD & $1.40 \mathrm{E}-01$ & $1.22 \mathrm{E}-01$ & $1.84 \mathrm{E}-02$ & $2.91 \mathrm{E}-01$ & $6.77 \mathrm{E}-01$ & $2.45 \mathrm{E}-01$ & $2.57 \mathrm{E}-01$ & $5.69 \mathrm{E}-02$ & $9.18 \mathrm{E}-02$ & $3.72 \mathrm{E}-02$ & $1.50 \mathrm{E}-01$ & $6.19 \mathrm{E}-02$ \\
\hline \multirow[t]{2}{*}{ Return } & $7.0097 \%$ & $7.1543 \%$ & $8.0669 \%$ & $11.4451 \%$ & $18.0109 \%$ & $9.4940 \%$ & $10.9893 \%$ & $14.1095 \%$ & $11.7183 \%$ & $7.7100 \%$ & $5.8630 \%$ & $8.0933 \%$ \\
\hline & (4.41E-03) & (3.65E-03) & $(2.58 \mathrm{E}-05)$ & $(1.42 \mathrm{E}-03)$ & (8.14E-03) & $(2.96 \mathrm{E}-04)$ & (8.67E-03) & $(7.27 \mathrm{E}-04)$ & $(4.83 \mathrm{E}-04)$ & $(2.46 \mathrm{E}-03)$ & (1.13E-03) & $(3.66 \mathrm{E}-04)$ \\
\hline $\mathrm{T} 1, \mathrm{~T} 2$ & 266,285 & 215,219 & 294,297 & 247,268 & 309,340 & $330,336^{*}$ & 193,198 & 313,345 & $330,336^{*}$ & 193,198 & 280,284 & 292,305 \\
\hline $\operatorname{Min} A D$ & 2.36E-07 & $1.53 \mathrm{E}-05$ & $7.42 \mathrm{E}-07$ & $6.09 \mathrm{E}-07$ & $1.08 \mathrm{E}-06$ & $4.10 \mathrm{E}-06$ & $2.20 \mathrm{E}-07$ & $5.77 \mathrm{E}-07$ & $3.56 \mathrm{E}-06$ & $4.83 \mathrm{E}-07$ & $7.50 \mathrm{E}-06$ & 8.32E-06 \\
\hline MinRD & 2.89E-06 & $1.88 \mathrm{E}-04$ & $9.11 \mathrm{E}-06$ & $6.87 \mathrm{E}-06$ & $1.01 \mathrm{E}-05$ & $3.70 \mathrm{E}-05$ & $1.49 \mathrm{E}-06$ & 4.32E-06 & $2.76 \mathrm{E}-05$ & $8.58 \mathrm{E}-06$ & $1.09 \mathrm{E}-04$ & $1.09 \mathrm{E}-04$ \\
\hline \multirow[t]{2}{*}{ Return } & $8.1523 \%$ & $8.1530 \%$ & $8.1466 \%$ & $8.8666 \%$ & $10.7389 \%$ & $11.0986 \%$ & $14.7832 \%$ & $13.3494 \%$ & $12.9033 \%$ & $5.6248 \%$ & $6.8986 \%$ & $7.6226 \%$ \\
\hline & (3.24E-06) & (1.73E-04) & (3.34E-06) & (7.99E-06) & $(2.55 \mathrm{E}-05)$ & $(3.85 \mathrm{E}-05)$ & $(4.31 \mathrm{E}-06)$ & $(2.62 \mathrm{E}-06)$ & (7.05E-04) & $(6.41 \mathrm{E}-06)$ & $(2.24 \mathrm{E}-05)$ & $(2.52 \mathrm{E}-05)$ \\
\hline $\mathrm{T} 1, \mathrm{~T} 2$ & $12,13^{*}$ & $77,78^{*}$ & 160,306 & $12,13^{*}$ & 69,213 & 292,305 & $12,13^{*}$ & 67,277 & 122,126 & $12,13^{*}$ & 69,117 & 186,244 \\
\hline
\end{tabular}

* Bullet portfolio; ** Barbell portfolio; *** Both bullet and barbell portfolios 
Table 7 - Continued

\begin{tabular}{|c|c|c|c|c|c|c|c|c|c|c|c|c|}
\hline \multicolumn{13}{|c|}{ Panel B: Fisher-Weil Duration } \\
\hline & \multicolumn{3}{|c|}{$\begin{array}{c}\text { Flat } \\
\text { (August, 1989) }\end{array}$} & \multicolumn{3}{|c|}{$\begin{array}{c}\text { Increasing } \\
\text { (January, 1985) }\end{array}$} & \multicolumn{3}{|c|}{$\begin{array}{l}\text { Decreasing } \\
(\text { May, 1981) }\end{array}$} & \multicolumn{3}{|c|}{$\begin{array}{c}\text { Humped } \\
\text { (October, 1986) }\end{array}$} \\
\hline & 1 year & 5 year & 10 year & 1 year & 5 year & 10 year & 1 year & 5 year & 10 year & 1 year & 5 year & 10 year \\
\hline$\%$ Successful & $99.02 \%$ & $98.04 \%$ & $91.18 \%$ & $97.06 \%$ & $97.06 \%$ & $49.02 \%$ & $96.08 \%$ & $97.06 \%$ & $84.31 \%$ & $98.04 \%$ & $98.04 \%$ & $63.73 \%$ \\
\hline Within 100 basis points & $97.06 \%$ & $99.02 \%$ & $100 \%$ & $89.22 \%$ & $98.04 \%$ & $100 \%$ & $96.08 \%$ & $99.02 \%$ & $100 \%$ & $84.31 \%$ & $99.02 \%$ & $99.02 \%$ \\
\hline Within 1 basis point & $6.86 \%^{* * *}$ & $13.73 \%^{*}$ & $7.84 \%$ & $4.90 \%^{* * *}$ & $12.75 \%$ & $4.90 \%$ & $6.86 \%^{* * *}$ & $18.63 \%$ & $16.67 \%$ & $4.90 \%{ }^{* * *}$ & $9.80 \%$ & $3.92 \%$ \\
\hline MaxAD & $1.19 \mathrm{E}-02$ & $1.46 \mathrm{E}-02$ & $3.95 \mathrm{E}-03$ & $2.58 \mathrm{E}-02$ & $1.53 \mathrm{E}-02$ & $5.77 \mathrm{E}-03$ & $2.38 \mathrm{E}-02$ & $7.60 \mathrm{E}-03$ & $3.45 \mathrm{E}-03$ & $3.26 \mathrm{E}-02$ & $1.01 \mathrm{E}-02$ & $1.35 \mathrm{E}-02$ \\
\hline $\operatorname{MaxRD}$ & $1.45 \mathrm{E}-01$ & $1.79 \mathrm{E}-01$ & $4.85 \mathrm{E}-02$ & $2.91 \mathrm{E}-01$ & $1.43 \mathrm{E}-01$ & $5.20 \mathrm{E}-02$ & $1.61 \mathrm{E}-01$ & $5.69 \mathrm{E}-02$ & $2.67 \mathrm{E}-02$ & $5.80 \mathrm{E}-01$ & $1.46 \mathrm{E}-01$ & $1.77 \mathrm{E}-01$ \\
\hline \multirow[t]{2}{*}{ Return } & $9.3381 \%$ & $6.6978 \%$ & $8.5420 \%$ & $11.4451 \%$ & $12.2733 \%$ & $11.6764 \%$ & $17.1676 \%$ & $14.1095 \%$ & $13.2482 \%$ & $2.3639 \%$ & $7.9101 \%$ & $6.2751 \%$ \\
\hline & (1.40E-04) & $(8.12 \mathrm{E}-03)$ & (8.98E-05) & $(1.42 \mathrm{E}-03)$ & (3.62E-04) & $(5.50 \mathrm{E}-03)$ & (1.91E-03) & $(7.27 \mathrm{E}-04)$ & $(2.55 \mathrm{E}-04)$ & (1.95E-03) & $(5.86 \mathrm{E}-05)$ & (7.39E-03) \\
\hline $\mathrm{T} 1, \mathrm{~T} 2$ & 308,345 & 215,219 & 320,346 & 247,268 & 277,294 & 273,317 & 196,233 & 313,345 & $330,336^{*}$ & 266,285 & 313,345 & 292,301 \\
\hline $\operatorname{Min} A D$ & $2.32 \mathrm{E}-07$ & $1.16 \mathrm{E}-06$ & $3.90 \mathrm{E}-06$ & $6.09 \mathrm{E}-07$ & 5.79E-06 & $3.77 \mathrm{E}-05$ & $2.21 \mathrm{E}-07$ & $5.77 \mathrm{E}-07$ & $1.49 \mathrm{E}-05$ & 4.81E-07 & $5.26 \mathrm{E}-06$ & 7.89E-06 \\
\hline MinRD & $2.85 \mathrm{E}-06$ & $1.42 \mathrm{E}-05$ & $4.78 \mathrm{E}-05$ & $6.87 \mathrm{E}-06$ & $5.39 \mathrm{E}-05$ & $3.40 \mathrm{E}-4$ & $2.61 \mathrm{E}-05$ & $4.32 \mathrm{E}-06$ & $1.15 \mathrm{E}-04$ & $8.56 \mathrm{E}-06$ & $7.62 \mathrm{E}-05$ & $1.04 \mathrm{E}-04$ \\
\hline \multirow[t]{2}{*}{ Return } & $8.1523 \%$ & $8.1547 \%$ & $8.1469 \%$ & $8.8666 \%$ & $10.7394 \%$ & $11.0952 \%$ & $14.7832 \%$ & $13.3494 \%$ & $12.9051 \%$ & $5.6248 \%$ & $6.8989 \%$ & $7.6225 \%$ \\
\hline & $(3.23 \mathrm{E}-06)$ & $(1.43 \mathrm{E}-06)$ & $(1.03 \mathrm{E}-05)$ & (7.99E-06) & $(2.55 \mathrm{E}-05)$ & $(1.17 \mathrm{E}-05)$ & $(4.32 \mathrm{E}-06)$ & $(2.62 \mathrm{E}-06)$ & $(8.21 \mathrm{E}-06)$ & $(6.40 \mathrm{E}-06)$ & $(2.24 \mathrm{E}-05)$ & $(5.19 \mathrm{E}-06)$ \\
\hline $\mathrm{T} 1, \mathrm{~T} 2$ & $12,13^{*}$ & 69,117 & 160,306 & $12,13^{*}$ & 69,213 & 211,244 & $12,13^{*}$ & 67,277 & 186,312 & $12,13^{*}$ & 69,117 & 181,229 \\
\hline
\end{tabular}

Bullet portfolio

** Barbell portfolio

*** Both barbell and bullet portfolios 
reasons for this poor performance. In theory, Macaulay's duration is not expected to provide successful immunization when the yield curve is not flat. Nonetheless, Macaulay's duration performs well for short and medium holding periods irrespective of the yield curve shape and the volatility. If the volatility is high, however, the deterioration in the performance for long holding periods can be due to using the duration matching strategy rather than the duration and convexity matching strategy. When the forward rate volatilities are high, the convexity adjustment is more important for the long holding periods because of the positive relation between convexity and time to maturity.

If this hypothesis is correct, then we should observe a similar performance with FisherWeil duration in the long run under high forward rate volatilities. If Fisher-Weil duration performs better than Macaulay's duration, then Macaulay's duration might be a poor risk measure for yield curves that are not flat, as the theory suggests. In this respect, if Fisher-Weil duration does not provide better performance than Macaulay's duration in the long run under high volatility, then the problem is not due to the yield curve shape or Macaulay's duration but rather due to the choice of the immunization strategy.

As we observe from Table 7, Panel A, in short holding periods, bullet portfolios produce the closest returns to the target yield. In medium to long holding periods, when more than $95 \%$ of the random portfolios are successful, bullet and barbell portfolios are also successful. We observe unsuccessful bullet portfolios for the ten-year holding period under a flat initial yield curve. In this respect, we cannot favor bullet and barbell portfolios to random portfolios for medium to long holding periods. For short holding periods, bullet portfolios are successful under both active and passive portfolio management and therefore can be preferred to other alternatives.

As shown in Table 7 Panel B, the performance of Fisher-Weil duration is similar to the performance of Macaulay's duration. Fisher-Weil performs well for short to long holding periods, but its performance deteriorates in the long run if the forward rate volatility is relatively high. Under increasing and humped initial yield curves, which correspond to high forward rate volatilities, less than $64 \%$ of the portfolios are successfully immunized according to the active portfolio management criterion. More than $85 \%$ of the portfolios are within 100 
basis of the target yield, but less than $19 \%$ of the portfolios are within one basis point of the target yield.

Since these results are very similar to those found by Macaulay's duration, we can question the duration matching strategy rather than the risk measures when the forward rates volatilities are high and the holding period is long. In this respect, in the following chapter we examine the performance of duration and convexity matching strategies to observe the affects of convexity adjustment.

With Fisher-Weil duration, again bullet portfolios produce the closest returns to the target portfolio for short holding periods. There are cases where bullet and barbell portfolios do not perform well for long holding periods. Therefore, we cannot favor any portfolio formation strategy for medium to long holding periods. For the short run, however, bullet portfolios perform well under both active and passive portfolio management and therefore can be preferred to the other alternatives.

Table 8 confirms the results related to the bullet and barbell portfolios. These portfolios produce returns close to the target yield for short holding periods but their performance deteriorates as the holding period increases. These results hold for both Macaulay's and Fisher-Weil duration. The bullet portfolios produce closer returns to the target than the barbell portfolios only over short holding periods. The performances of bullet and barbell portfolios are similar for medium to long holding periods.

\subsection{Exponential Decay Volatility One-Factor HJM Framework}

The performances of the duration-matched portfolios under the exponential decay volatility HJM framework are summarized in Table 9. Panels A, B, and C present the performances of the portfolios matched with Macaulay's, Fisher-Weil, and exponential decay HJM durations, respectively.

As observed from Table 9 Panel A, for short and medium holding periods, Macaulay's duration is successful for all yield curve shapes under the active portfolio management 


\section{Table 8}

\section{Performance of Duration Matched Bullet and Barbell Portfolios under Constant Volatility HJM Framework}

This table shows the performance of duration matched bullet and barbell portfolios under constant volatility HJM framework. Bullet and barbell portfolios are duration matched using Macaulay's and Fisher-Weil duration measures. HJM duration measure reduces to Fisher-Weil duration measure under constant volatility HJM framework. The results are analyzed according to the initial yield curves and holding periods considered. Initial yield curves considered in the study are flat, increasing, decreasing, and humped yield curves. Holding periods are one, five and ten years. For each portfolio matched with a duration measure, the first row shows the holding period return. Standard errors of the returns are in parenthesis. The following two rows are the maximum absolute (MaxAD) and relative (MaxRD) deviations of portfolio returns from the target return.

\begin{tabular}{|c|c|c|c|c|c|c|c|c|c|c|c|c|}
\hline & \multicolumn{3}{|c|}{$\begin{array}{c}\text { Flat } \\
\text { (August, 1989) }\end{array}$} & \multicolumn{3}{|c|}{$\begin{array}{c}\text { Increasing } \\
\text { (January, 1985) }\end{array}$} & \multicolumn{3}{|c|}{$\begin{array}{l}\text { Decreasing } \\
(\text { May, 1981) }\end{array}$} & \multicolumn{3}{|c|}{$\begin{array}{c}\text { Humped } \\
\text { (October, 1986) } \\
\end{array}$} \\
\hline & 1 year & 5 year & 10 year & 1 year & 5 year & 10 year & 1 year & 5 year & 10 year & 1 year & 5 year & 10 year \\
\hline \multicolumn{13}{|l|}{ Bullet } \\
\hline Macaulay & $\begin{array}{c}8.1523 \% \\
(3.24 \mathrm{E}-06)\end{array}$ & $\begin{array}{c}8.1530 \% \\
(1.73 \mathrm{E}-03)\end{array}$ & $\begin{array}{c}8.0833 \% \\
(6.89 \mathrm{E}-05)\end{array}$ & $\begin{array}{c}8.8666 \% \\
(7.99 \mathrm{E}-06)\end{array}$ & $\begin{array}{r}10.7255 \% \\
(1.49 \mathrm{E}-04)\end{array}$ & $\begin{array}{c}9.4940 \% \\
(2.96 \mathrm{E}-04)\end{array}$ & $\begin{array}{c}14.7832 \% \\
(4.31 \mathrm{E}-06)\end{array}$ & $\begin{array}{l}13.3426 \% \\
(2.47 \mathrm{E}-04)\end{array}$ & $\begin{array}{l}11.7183 \% \\
(4.83 \mathrm{E}-04)\end{array}$ & $\begin{array}{c}5.6248 \% \\
(6.41 \mathrm{E}-06)\end{array}$ & $\begin{array}{c}6.8868 \% \\
(4.87 \mathrm{E}-04)\end{array}$ & $\begin{array}{c}7.4770 \% \\
(7.38 \mathrm{E}-05)\end{array}$ \\
\hline MaxAD & $2.36 \mathrm{E}-07$ & $1.53 \mathrm{E}-05$ & $6.32 \mathrm{E}-04$ & $6.09 \mathrm{E}-07$ & $1.33 \mathrm{E}-04$ & $1.60 \mathrm{E}-03$ & $2.20 \mathrm{E}-07$ & $6.84 \mathrm{E}-05$ & $1.19 \mathrm{E}-02$ & $4.83 \mathrm{E}-07$ & $1.26 \mathrm{E}-04$ & $1.45 \mathrm{E}-03$ \\
\hline MaxRD & $2.89 \mathrm{E}-06$ & $1.88 \mathrm{E}-04$ & 7.82E-03 & $6.87 \mathrm{E}-06$ & $1.24 \mathrm{E}-03$ & $1.45 \mathrm{E}-02$ & $1.49 \mathrm{E}-06$ & $5.12 \mathrm{E}-04$ & $9.19 \mathrm{E}-02$ & $8.58 \mathrm{E}-06$ & $1.82 \mathrm{E}-03$ & $1.90 \mathrm{E}-02$ \\
\hline Fisher-Weil & $\begin{array}{c}8.1523 \% \\
(3.23 \mathrm{E}-06)\end{array}$ & $\begin{array}{c}8.1541 \% \\
(5.51 \mathrm{E}-04)\end{array}$ & $\begin{array}{c}8.0855 \% \\
(6.85 \mathrm{E}-05)\end{array}$ & $\begin{array}{c}8.8666 \% \\
(7.97 \mathrm{E}-06)\end{array}$ & $\begin{array}{c}10.7257 \% \\
(1.49 E-04)\end{array}$ & $\begin{array}{l}11.6379 \% \\
(3.33 \mathrm{E}-04)\end{array}$ & $\begin{array}{l}14.7832 \% \\
(4.32 \mathrm{E}-06)\end{array}$ & $\begin{array}{r}13.3425 \% \\
(2.47 \mathrm{E}-04)\end{array}$ & $\begin{array}{l}13.2482 \% \\
(2.55 \mathrm{E}-04)\end{array}$ & $\begin{array}{c}5.6248 \% \\
(6.40 \mathrm{E}-06)\end{array}$ & $\begin{array}{c}6.8869 \% \\
(4.90 \mathrm{E}-04)\end{array}$ & $\begin{array}{c}7.4905 \% \\
(7.04 \mathrm{E}-05)\end{array}$ \\
\hline $\begin{array}{l}\text { MaxAD } \\
\text { MaxRD }\end{array}$ & $\begin{array}{l}2.32 \mathrm{E}-07 \\
2.85 \mathrm{E}-06\end{array}$ & $\begin{array}{l}4.87 \mathrm{E}-06 \\
5.97 \mathrm{E}-05\end{array}$ & $\begin{array}{l}6.10 \mathrm{E}-04 \\
7.54 \mathrm{E}-03\end{array}$ & $\begin{array}{l}6.07 \mathrm{E}-07 \\
6.85 \mathrm{E}-06\end{array}$ & $\begin{array}{l}1.31 \mathrm{E}-04 \\
1.22 \mathrm{E}-03\end{array}$ & $\begin{array}{l}5.39 \mathrm{E}-03 \\
4.86 \mathrm{E}-02\end{array}$ & $\begin{array}{l}2.21 \mathrm{E}-07 \\
1.49 \mathrm{E}-06\end{array}$ & $\begin{array}{l}6.93 \mathrm{E}-05 \\
5.19 \mathrm{E}-04\end{array}$ & $\begin{array}{l}3.45 \mathrm{E}-03 \\
2.67 \mathrm{E}-02\end{array}$ & $\begin{array}{l}4.81 \mathrm{E}-07 \\
8.56 \mathrm{E}-06\end{array}$ & $\begin{array}{l}1.25 \mathrm{E}-04 \\
1.81 \mathrm{E}-03\end{array}$ & $\begin{array}{l}1.31 \mathrm{E}-03 \\
1.72 \mathrm{E}-02\end{array}$ \\
\hline \multicolumn{13}{|l|}{ Barbell } \\
\hline Macaulay & $\begin{array}{c}8.1518 \% \\
(6.86 \mathrm{E}-08)\end{array}$ & $\begin{array}{c}8.1403 \% \\
(9.95 \mathrm{E}-07)\end{array}$ & $\begin{array}{c}8.0972 \% \\
(5.61 \mathrm{E}-06)\end{array}$ & $\begin{array}{c}8.8656 \% \\
(1.48 \mathrm{E}-06)\end{array}$ & $\begin{array}{c}10.7066 \% \\
(7.56 \mathrm{E}-06)\end{array}$ & $\begin{array}{r}10.9850 \% \\
(9.02 \mathrm{E}-06)\end{array}$ & $\begin{array}{r}14.7829 \% \\
(8.61 \mathrm{E}-07)\end{array}$ & $\begin{array}{r}13.3371 \% \\
(5.05 \mathrm{E}-06)\end{array}$ & $\begin{array}{l}12.8579 \% \\
(1.67 \mathrm{E}-05)\end{array}$ & $\begin{array}{c}5.6238 \% \\
(1.29 \mathrm{E}-06)\end{array}$ & $\begin{array}{c}6.8707 \% \\
(6.62 \mathrm{E}-06)\end{array}$ & $\begin{array}{c}7.5188 \% \\
(1.31 \mathrm{E}-05)\end{array}$ \\
\hline $\begin{array}{l}\text { MaxAD } \\
\text { MaxRD }\end{array}$ & $\begin{array}{l}4.91 \mathrm{E}-06 \\
6.03 \mathrm{E}-05\end{array}$ & $\begin{array}{l}1.43 \mathrm{E}-04 \\
1.75 \mathrm{E}-03\end{array}$ & $\begin{array}{l}1.93 \mathrm{E}-04 \\
6.10 \mathrm{E}-03\end{array}$ & $\begin{array}{l}1.04 \mathrm{E}-05 \\
1.17 \mathrm{E}-04\end{array}$ & $\begin{array}{l}3.21 \mathrm{E}-04 \\
2.99 \mathrm{E}-03\end{array}$ & $\begin{array}{l}1.14 \mathrm{E}-03 \\
1.03 \mathrm{E}-02\end{array}$ & $\begin{array}{l}3.69 \mathrm{E}-06 \\
2.50 \mathrm{E}-05\end{array}$ & $\begin{array}{l}1.23 \mathrm{E}-04 \\
9.24 \mathrm{E}-04\end{array}$ & $\begin{array}{l}4.58 \mathrm{E}-04 \\
3.55 \mathrm{E}-03\end{array}$ & $\begin{array}{l}1.00 \mathrm{E}-05 \\
1.79 \mathrm{E}-04\end{array}$ & $\begin{array}{l}2.87 \mathrm{E}-04 \\
4.16 \mathrm{E}-03\end{array}$ & $\begin{array}{l}1.03 \mathrm{E}-03 \\
1.35 \mathrm{E}-02\end{array}$ \\
\hline Fisher-Weil & $\begin{array}{c}8.1518 \% \\
(4.80 \mathrm{E}-08)\end{array}$ & $\begin{array}{c}8.1404 \% \\
(1.74 \mathrm{E}-07)\end{array}$ & $\begin{array}{c}8.0984 \% \\
(5.12 \mathrm{E}-06)\end{array}$ & $\begin{array}{c}8.8656 \% \\
(1.48 \mathrm{E}-06)\end{array}$ & $\begin{array}{c}10.7067 \% \\
(7.57 \mathrm{E}-06)\end{array}$ & $\begin{array}{r}10.9829 \% \\
(9.11 \mathrm{E}-06)\end{array}$ & $\begin{array}{c}14.7829 \% \\
(8.59 \mathrm{E}-07)\end{array}$ & $\begin{array}{c}13.3361 \% \\
(4.66 \mathrm{E}-06)\end{array}$ & $\begin{array}{l}12.8505 \% \\
(6.27 \mathrm{E}-06)\end{array}$ & $\begin{array}{c}5.6239 \% \\
(1.29 \mathrm{E}-06)\end{array}$ & $\begin{array}{c}6.8721 \% \\
(6.25 \mathrm{E}-06)\end{array}$ & $\begin{array}{c}7.5279 \% \\
(7.38 \mathrm{E}-06)\end{array}$ \\
\hline MaxAD & $4.88 \mathrm{E}-06$ & $1.41 \mathrm{E}-04$ & 4.81E-04 & $1.03 \mathrm{E}-05$ & $3.21 \mathrm{E}-04$ & $1.16 \mathrm{E}-03$ & $3.86 \mathrm{E}-06$ & $1.33 \mathrm{E}-04$ & $5.32 \mathrm{E}-04$ & $9.74 \mathrm{E}-06$ & 2.73E-04 & $9.38 \mathrm{E}-04$ \\
\hline MaxRD & $5.99 \mathrm{E}-05$ & $1.74 \mathrm{E}-03$ & $5.94 \mathrm{E}-03$ & $1.17 \mathrm{E}-04$ & $2.99 \mathrm{E}-03$ & $1.05 \mathrm{E}-02$ & $2.61 \mathrm{E}-05$ & $9.98 \mathrm{E}-04$ & $4.12 \mathrm{E}-03$ & $1.73 \mathrm{E}-04$ & $3.95 \mathrm{E}-03$ & $1.23 \mathrm{E}-02$ \\
\hline
\end{tabular}




\section{Table 9}

\section{Performance of Duration Matched Portfolios under Exponential Decay Volatility HJM Framework}

The performances of 102 portfolios are summarized. Two of the portfolios are bullet and barbell, and the rest are random portfolios. The benchmark portfolios are the ones that are matched according to the duration measure of the one factor HJM model with exponentially decaying volatility. The other two duration measures are the Macaulay's and the Fisher-Weil durations. Panel A, B, and C presents the results the performances of immunization portfolios matched by Macaulay's duration, Fisher-Weil duration, and the exponential decay volatility one factor HJM duration, respectively. The results are analyzed according to the initial yield curves and holding periods. Four initial yield curves are considered: Flat (August 1989); increasing (January 1985); decreasing (May 1981); and humped (October 1986). The three holding periods are one-, five-, and ten-years. Three criteria are used to assess the immunization performances of the portfolios. According to one criterion, a portfolio is considered successful in immunization if the holding period return of the portfolio is greater than or equal to the yield of the target zero coupon bond minus 5 basis points. In Panels A and B, "\% Successful" ("\%Unsuccessful") gives the percentage of the portfolios that are successful (unsuccessful) in immunization according to the first criterion. The other two criteria assess the immunization performances by measuring the percentage of portfolios with returns within one and 100 basis points of the target yield. These are given in the next two rows. The maximum and minimum absolute (MaxAD and MinAD) and relative (MaxRD and MinRD) deviations from the target return are presented next. For each deviation, the returns of these portfolios, and the time to maturity of the bonds in the portfolios are given. Standard errors of the returns are in parentheses. Times to maturity are given in months, (T1, T2)

\begin{tabular}{|c|c|c|c|c|c|c|c|c|c|c|c|c|}
\hline \multicolumn{13}{|c|}{ Panel A: Macaulay's Duration } \\
\hline & \multicolumn{3}{|c|}{$\begin{array}{c}\text { Flat } \\
\text { (August, 1989) } \\
\end{array}$} & \multicolumn{3}{|c|}{$\begin{array}{c}\text { Increasing } \\
\text { (January, 1985) }\end{array}$} & \multicolumn{3}{|c|}{$\begin{array}{l}\text { Decreasing } \\
(\text { May, 1981) }\end{array}$} & \multicolumn{3}{|c|}{$\begin{array}{c}\text { Humped } \\
\text { (October, 1986) }\end{array}$} \\
\hline & 1 year & 5 year & 10 year & 1 year & 5 year & 10 year & 1 year & 5 year & 10 year & 1 year & 5 year & 10 year \\
\hline$\%$ Successful & $98.04 \%$ & $98.04 \%$ & $70.59 \%$ & $97.06 \%$ & $98.04 \%$ & $14.71 \%$ & $97.06 \%$ & $98.04 \%$ & $92.16 \%$ & $98.04 \%$ & $98.04 \%$ & $25.49 \%$ \\
\hline Within 100 basis points & $99.02 \%$ & $100 \%$ & $100 \%$ & $92.16 \%$ & $97.06 \%$ & $99.02 \%$ & $97.06 \%$ & $100 \%$ & $100 \%$ & $94.12 \%$ & $99.02 \%$ & $99.02 \%$ \\
\hline Within 1 basis point & $8.83 \%{ }^{* * *}$ & $22.55 \%^{*}$ & $12.75 \%$ & $6.86 \%{ }^{* * *}$ & $16.67 \%$ & $0.98 \%$ & $9.80 \%^{* * *}$ & $20.59 \%{ }^{*}$ & $28.43 \%$ & $6.86 \%^{* * *}$ & $15.69 \%$ & $2.94 \%$ \\
\hline MaxAD & $1.27 \mathrm{E}-02$ & $6.41 \mathrm{E}-03$ & $9.76 \mathrm{E}-03$ & $3.02 \mathrm{E}-02$ & $9.11 \mathrm{E}-02$ & $1.22 \mathrm{E}-02$ & $3.21 \mathrm{E}-02$ & 7.07E-03 & $8.14 \mathrm{E}-03$ & $1.75 \mathrm{E}-02$ & $1.24 \mathrm{E}-02$ & $4.22 \mathrm{E}-03$ \\
\hline MaxRD & $1.56 \mathrm{E}-01$ & $7.85 \mathrm{E}-02$ & $1.20 \mathrm{E}-01$ & $3.41 \mathrm{E}-01$ & $8.48 \mathrm{E}-01$ & $1.10 \mathrm{E}-01$ & $2.17 \mathrm{E}-01$ & $5.30 \mathrm{E}-02$ & $6.31 \mathrm{E}-02$ & $3.12 \mathrm{E}-01$ & $1.79 \mathrm{E}-01$ & $5.53 \mathrm{E}-02$ \\
\hline Return & $\begin{array}{l}6.8809 \% \\
(9.49 \mathrm{E}-04)\end{array}$ & $\begin{array}{c}7.5140 \% \\
(8.43 \mathrm{E}-04)\end{array}$ & $\begin{array}{c}7.1703 \% \\
(9.90 \mathrm{E}-03)\end{array}$ & $\begin{array}{l}11.8899 \% \\
(1.37 \mathrm{E}-03)\end{array}$ & $\begin{array}{l}19.8464 \% \\
(2.41 \mathrm{E}-02)\end{array}$ & $\begin{array}{l}12.3146 \% \\
(5.17 \mathrm{E}-03)\end{array}$ & $\begin{array}{l}11.5746 \% \\
(8.01 \mathrm{E}-03)\end{array}$ & $\begin{array}{l}14.0566 \% \\
(7.67 \mathrm{E}-04)\end{array}$ & $\begin{array}{l}13.7175 \% \\
(1.19 \mathrm{E}-03)\end{array}$ & $\begin{array}{l}3.8704 \% \\
(1.63 \mathrm{E}-03)\end{array}$ & $\begin{array}{c}5.6629 \% \\
(8.15 \mathrm{E}-04)\end{array}$ & $\begin{array}{r}8.0433 \% \\
(8.71 \mathrm{E}-04)\end{array}$ \\
\hline $\mathrm{T} 1, \mathrm{~T} 2$ & 266,285 & 215,219 & 292,305 & 247,268 & 297,346 & 320,346 & 193,198 & 313,345 & 317,342 & 266,279 & 280,284 & 292,305 \\
\hline MinAD & $1.80 \mathrm{E}-07$ & $2.64 \mathrm{E}-06$ & 1.11 & 3.79E-07 & 3.79E-06 & $5.67 \mathrm{E}-05$ & $1.83 \mathrm{E}-07$ & $2.38 \mathrm{E}-06$ & 4.15 & E-07 & $5.20 \mathrm{E}-06$ & 1.3 \\
\hline MinRD & $2.20 \mathrm{E}-06$ & $3.24 \mathrm{E}-05$ & $1.36 \mathrm{E}-04$ & $4.27 \mathrm{E}-06$ & $3.53 \mathrm{E}-05$ & $5.11 \mathrm{E}-04$ & $1.24 \mathrm{E}-06$ & $1.79 \mathrm{E}-05$ & $3.21 \mathrm{E}-05$ & $5.76 \mathrm{E}-06$ & 7.54E-05 & $1.81 \mathrm{E}-04$ \\
\hline Return & $\begin{array}{l}8.1523 \% \\
(3.69 \mathrm{E}-06)\end{array}$ & $\begin{array}{l}8.1543 \% \\
(1.00 \mathrm{E}-05)\end{array}$ & $\begin{array}{c}8.1476 \% \\
(4.09 \mathrm{E}-05)\end{array}$ & $\begin{array}{l}8.8666 \% \\
(6.41 \mathrm{E}-06)\end{array}$ & $\begin{array}{l}10.7384 \% \\
(1.85 \mathrm{E}-05)\end{array}$ & $\begin{array}{l}11.1046 \% \\
(3.66 \mathrm{E}-05)\end{array}$ & $\begin{array}{c}14.7832 \% \\
(3.82 \mathrm{E}-06\end{array}$ & $\begin{array}{l}13.3497 \% \\
(1.43 \mathrm{E}-05)\end{array}$ & $\begin{array}{l}12.9032 \% \\
(2.67 \mathrm{E}-05)\end{array}$ & $\begin{array}{c}5.6248 \% \\
(5.29 \mathrm{E}-06)\end{array}$ & $\begin{array}{r}6.8989 \% \\
(1.91 \mathrm{E}-05)\end{array}$ & $\begin{array}{r}7.6204 \% \\
(1.07 \mathrm{E}-04)\end{array}$ \\
\hline $\mathrm{T} 1, \mathrm{~T} 2$ & $12,13^{*}$ & 71,196 & 212,292 & $12,13^{*}$ & 78,142 & 264,300 & $12,13^{*}$ & 69,277 & 205,325 & $12,13^{*}$ & 72,224 & 264,300 \\
\hline
\end{tabular}


Table 9 - Continued

\begin{tabular}{|c|c|c|c|c|c|c|c|c|c|c|c|c|}
\hline \multicolumn{13}{|c|}{ Panel B: Fisher-Weil Duration } \\
\hline & \multicolumn{3}{|c|}{$\begin{array}{c}\text { Flat } \\
\text { (August, 1989) }\end{array}$} & \multicolumn{3}{|c|}{$\begin{array}{c}\text { Increasing } \\
\text { (January, 1985) }\end{array}$} & \multicolumn{3}{|c|}{$\begin{array}{c}\text { Decreasing } \\
\text { (May, 1981) }\end{array}$} & \multicolumn{3}{|c|}{$\begin{array}{c}\text { Humped } \\
\text { (October, 1986) }\end{array}$} \\
\hline & 1 year & 5 year & 10 year & 1 year & 5 year & 10 year & 1 year & 5 year & 10 year & 1 year & 5 year & 10 year \\
\hline$\%$ Successful & $99.02 \%$ & $98.04 \%$ & $69.61 \%$ & $98.04 \%$ & $98.04 \%$ & $8.82 \%$ & $96.08 \%$ & $97.06 \%$ & $79.41 \%$ & $98.04 \%$ & $98.04 \%$ & $30.39 \%$ \\
\hline Within 100 basis points & $95.10 \%$ & $98.04 \%$ & $100 \%$ & $88.24 \%$ & $98.04 \%$ & $99.02 \%$ & $96.08 \%$ & $99.02 \%$ & $99.02 \%$ & $84.31 \%$ & $96.08 \%$ & $99.02 \%$ \\
\hline Within 1 basis point & $9.80 \%{ }^{* * *}$ & $22.55 \%^{*}$ & $13.73 \%$ & $6.86 \% \%^{* * *}$ & $16.67 \%$ & $1.96 \%$ & $9.80 \%{ }^{* * *}$ & $21.57 \%^{*}$ & $12.75 \%$ & $6.86 \%{ }^{* * *}$ & $14.71 \%$ & $1.96 \%$ \\
\hline MaxAD & $1.70 \mathrm{E}-02$ & $1.18 \mathrm{E}-02$ & 4.23E-03 & 3.77E-02 & $1.61 \mathrm{E}-02$ & $1.13 \mathrm{E}-02$ & $3.02 \mathrm{E}-02$ & 4.25E-02 & $3.43 \mathrm{E}-03$ & 4.13E-02 & $1.45 \mathrm{E}-02$ & 2.09E-02 \\
\hline MaxRD & $2.08 \mathrm{E}-01$ & $1.45 \mathrm{E}-01$ & $5.19 \mathrm{E}-02$ & $4.25 \mathrm{E}-01$ & $1.50 \mathrm{E}-01$ & $1.02 \mathrm{E}-01$ & $2.04 \mathrm{E}-01$ & $3.19 \mathrm{E}-01$ & $2.70 \mathrm{E}-02$ & 7.34E-01 & $2.10 \mathrm{E}-01$ & $2.74 \mathrm{E}-01$ \\
\hline Return & $\begin{array}{c}9.8499 \% \\
(7.77 \mathrm{E}-04)\end{array}$ & $\begin{array}{c}9.3367 \% \\
(6.87 \mathrm{E}-04)\end{array}$ & $\begin{array}{c}7.7234 \% \\
(4.14 \mathrm{E}-03)\end{array}$ & $\begin{array}{l}5.0970 \% \\
(1.67 \mathrm{E}-03)\end{array}$ & $\begin{array}{l}12.3483 \% \\
(8.57 \mathrm{E}-04)\end{array}$ & $\begin{array}{l}9.9653 \% \\
(6.40 \mathrm{E}-03)\end{array}$ & $\begin{array}{l}11.7649 \% \\
(4.82 \mathrm{E}-03)\end{array}$ & $\begin{array}{l}17.6033 \% \\
(3.56 \mathrm{E}-03)\end{array}$ & $\begin{array}{l}13.2524 \% \\
(3.89 \mathrm{E}-04)\end{array}$ & $\begin{array}{c}1.4962 \% \% \\
(1.76 \mathrm{E}-03)\end{array}$ & $\begin{array}{c}8.3515 \% \\
(5.48 \mathrm{E}-04)\end{array}$ & $\begin{array}{c}5.5315 \% \\
(7.86 \mathrm{E}-03)\end{array}$ \\
\hline $\mathrm{T} 1, \mathrm{~T} 2$ & 308,345 & 309,340 & 292,301 & 247,268 & 277,294 & 307,346 & 288,345 & 240,277 & 307,346 & 266,285 & 313,345 & 292,305 \\
\hline $\operatorname{Min} A D$ & $1.80 \mathrm{E}-07$ & $2.67 \mathrm{E}-06$ & $1.95 \mathrm{E}-05$ & $3.78 \mathrm{E}-07$ & $1.74 \mathrm{E}-06$ & $8.78 \mathrm{E}-08$ & $1.84 \mathrm{E}-07$ & $1.54 \mathrm{E}-06$ & $6.37 \mathrm{E}-06$ & $3.24 \mathrm{E}-07$ & $1.30 \mathrm{E}-06$ & $2.56 \mathrm{E}-05$ \\
\hline MinRD & $2.20 \mathrm{E}-06$ & $3.28 \mathrm{E}-05$ & $2.40 \mathrm{E}-04$ & $4.26 \mathrm{E}-06$ & $1.62 \mathrm{E}-05$ & 7.91E-07 & $1.24 \mathrm{E}-06$ & $1.15 \mathrm{E}-05$ & 4.94E-05 & $5.76 \mathrm{E}-06$ & $1.88 \mathrm{E}-05$ & $3.35 \mathrm{E}-04$ \\
\hline \multirow[t]{2}{*}{ Return } & $8.1523 \%$ & $8.1543 \%$ & $8.1446 \%$ & $8.8666 \%$ & $10.7386 \%$ & $11.0990 \%$ & $14.7832 \%$ & $13.3493 \%$ & $12.9043 \%$ & $5.6248 \%$ & $6.8993 \%$ & $7.6192 \%$ \\
\hline & $(3.69 \mathrm{E}-06)$ & $(1.01 \mathrm{E}-05)$ & (4.08E-05) & (6.39E-06) & $(1.81 E-05)$ & $(5.47 \mathrm{E}-05)$ & (3.82E-06 & $(1.46 \mathrm{E}-05)$ & (1.99E-05) & $(5.28 \mathrm{E}-06)$ & $(1.88 \mathrm{E}-05)$ & (6.60E-05) \\
\hline $\mathrm{T} 1, \mathrm{~T} 2$ & $12,13^{*}$ & 71,196 & 207,292 & $12,13^{*}$ & 78,142 & 269,323 & $12,13^{*}$ & 69,277 & 210,338 & $12,13^{*}$ & 72,224 & 220,330 \\
\hline
\end{tabular}


Table 9 - Continued

\begin{tabular}{|c|c|c|c|c|c|c|c|c|c|c|c|c|}
\hline \multicolumn{13}{|c|}{ Panel C: HJM Exponential Decay Duration } \\
\hline & \multicolumn{3}{|c|}{$\begin{array}{c}\text { Flat } \\
\text { (August, 1989) }\end{array}$} & \multicolumn{3}{|c|}{$\begin{array}{c}\text { Increasing } \\
\text { (January, 1985) }\end{array}$} & \multicolumn{3}{|c|}{$\begin{array}{c}\text { Decreasing } \\
(\text { May, 1981) }\end{array}$} & \multicolumn{3}{|c|}{$\begin{array}{c}\text { Humped } \\
\text { (October, 1986) }\end{array}$} \\
\hline & 1 year & 5 year & 10 year & 1 year & 5 year & 10 year & 1 year & 5 year & 10 year & 1 year & 5 year & 10 year \\
\hline$\%$ Successful & $98.04 \%$ & $98.04 \%$ & $97.06 \%$ & $99.02 \%$ & $96.08 \%$ & $81.37 \%$ & $98.04 \%$ & $97.06 \%$ & $94.12 \%$ & $99.02 \%$ & $99.02 \%$ & $94.12 \%$ \\
\hline Within 100 basis points & $100 \%$ & $99.02 \%$ & $100 \%$ & $97.06 \%$ & $98.04 \%$ & $100 \%$ & $98.04 \%$ & $100 \%$ & $99.02 \%$ & $95.10 \%$ & $99.02 \%$ & $100 \%$ \\
\hline Within 1 basis point & $9.80 \%{ }^{* * *}$ & $21.57 \%{ }^{* * *}$ & $14.71 \%$ & $7.84 \%{ }^{* * *}$ & $19.61 \%^{*}$ & $6.86 \%$ & $9.80 \%^{* * *}$ & $21.57 \%{ }^{* * *}$ & $19.61 \%$ & $7.84 \%{ }^{* * *}$ & $17.65 \%^{*}$ & $9.80 \%$ \\
\hline MaxAD & $7.41 \mathrm{E}-03$ & $1.07 \mathrm{E}-02$ & $3.56 \mathrm{E}-03$ & $2.31 \mathrm{E}-02$ & $1.61 \mathrm{E}-02$ & $7.15 \mathrm{E}-03$ & $5.56 \mathrm{E}-02$ & $5.57 \mathrm{E}-03$ & $1.53 \mathrm{E}-02$ & $2.94 \mathrm{E}-02$ & $3.14 \mathrm{E}-02$ & $4.52 \mathrm{E}-03$ \\
\hline MaxRD & $9.08 \mathrm{E}-02$ & $1.31 \mathrm{E}-01$ & $4.36 \mathrm{E}-02$ & $2.61 \mathrm{E}-01$ & $1.50 \mathrm{E}-01$ & $6.44 \mathrm{E}-02$ & $3.76 \mathrm{E}-01$ & 4.17E-02 & $1.19 \mathrm{E}-01$ & $5.23 \mathrm{E}-01$ & $4.55 \mathrm{E}-01$ & $5.92 \mathrm{E}-02$ \\
\hline Return & $\begin{array}{l}8.8929 \% \\
(4.19 \mathrm{E}-04)\end{array}$ & $\begin{array}{l}7.0861 \% \\
(6.93 \mathrm{E}-03)\end{array}$ & $\begin{array}{c}7.7909 \% \\
(5.17 \mathrm{E}-03)\end{array}$ & $\begin{array}{l}11.1804 \% \\
(4.18 \mathrm{E}-04)\end{array}$ & $\begin{array}{l}12.3484 \% \\
(2.77 \mathrm{E}-03)\end{array}$ & $\begin{array}{l}11.8138 \% \\
(4.73 \mathrm{E}-04)\end{array}$ & $\begin{array}{l}9.2216 \% \\
(1.55 \mathrm{E}-03)\end{array}$ & $\begin{array}{l}13.9068 \% \\
(8.30 \mathrm{E}-03)\end{array}$ & $\begin{array}{l}14.4352 \% \\
(1.37 \mathrm{E}-03)\end{array}$ & $\begin{array}{c}8.5663 \% \\
(2.42 \mathrm{E}-03)\end{array}$ & $\begin{array}{l}10.0409 \% \\
(6.01 \mathrm{E}-03)\end{array}$ & $\begin{array}{l}8.0732 \% \\
(2.63 \mathrm{E}-04)\end{array}$ \\
\hline $\mathrm{T} 1, \mathrm{~T} 2$ & 266,285 & 215,219 & 292,305 & 288,345 & 297,346 & 307,346 & 193,198 & 284,315 & $336,342 *$ & 266,279 & 215,219 & 292,305 \\
\hline $\operatorname{Min} A D$ & 7.23E-08 & $6.96 \mathrm{E}-07$ & $6.43 \mathrm{E}-06$ & $1.61 \mathrm{E}-07$ & 4.94E-06 & $2.05 \mathrm{E}-05$ & $1.16 \mathrm{E}-07$ & $1.86 \mathrm{E}-06$ & $1.17 \mathrm{E}-05$ & $1.21 \mathrm{E}-07$ & $1.23 \mathrm{E}-06$ & $7.74 \mathrm{E}-06$ \\
\hline MinRD & $2.20 \mathrm{E}-06$ & $8.53 \mathrm{E}-06$ & 7.89E-05 & $1.81 \mathrm{E}-06$ & $4.60 \mathrm{E}-05$ & $1.85 \mathrm{E}-04$ & $7.86 \mathrm{E}-07$ & $1.40 \mathrm{E}-05$ & $9.06 \mathrm{E}-05$ & $2.15 \mathrm{E}-06$ & $1.79 \mathrm{E}-05$ & $1.01 \mathrm{E}-04$ \\
\hline Return & $8.1523 \%$ & $8.1546 \%$ & $8.1472 \%$ & $8.8666 \%$ & $10.7393 \%$ & $11.1010 \%$ & $14.7832 \%$ & $13.3493 \%$ & $12.9024 \%$ & $5.6248 \%$ & $6.8993 \%$ & $7.6210 \%$ \\
\hline & $(2.37 \mathrm{E}-06)$ & $(1.02 \mathrm{E}-05)$ & $(5.73 \mathrm{E}-06)$ & $(4.08 \mathrm{E}-06)$ & $(1.45 \mathrm{E}-05)$ & $(8.78 \mathrm{E}-06)$ & $(3.12 \mathrm{E}-06)$ & $(1.35 \mathrm{E}-05)$ & $(7.58 \mathrm{E}-06)$ & $(3.20 \mathrm{E}-06)$ & $(1.28 \mathrm{E}-05)$ & $(1.01 \mathrm{E}-05)$ \\
\hline $\mathrm{T} 1, \mathrm{~T} 2$ & $12,13^{*}$ & 69,117 & 177,212 & $12,13^{*}$ & 69,213 & 200,249 & $12,13^{*}$ & 67,302 & 200,249 & $12,13^{*}$ & 69,117 & 174,230 \\
\hline
\end{tabular}

Bullet portfolio

** Barbell portfolio

*** Both bullet and barbell portfolios 
criterion. More than $97 \%$ of the portfolios are successfully immunized for these holding periods. The immunization performance of Macaulay's duration deteriorates for long holding periods. This deterioration is severe especially when the forward rate volatilities are high. As can be observed from Table 1 Panel C, forward rate volatilities are relatively high for ten-year holding periods under increasing and humped initial yield curves. Only 15\% of the portfolios under an increasing initial yield curve and $25 \%$ of the portfolios under a humped initial yield curve are successfully immunized for long holding periods. This deterioration in long holding periods especially under high volatility can have two alternative explanations. When the volatility is high and the holding period is long, Macaulay's duration might not be a good risk measure or rather the duration matching strategy is not the right immunization strategy under these circumstances. In these respects, if the Fisher-Weil and HJM risk measures perform better than Macaulay's duration, this would suggest that Macaulay's duration is a poor risk measure under high volatilities and long holding periods. If not, then we should analyze alternative immunization strategies. These analysis is carried out in the next chapters.

Under a passive portfolio management criterion, more than $93 \%$ of the portfolios are within 100 basis points of the target. On the other hand, for short holding periods, less than $10 \%$ of the portfolios and for medium holding periods, $17 \%$ to $21 \%$ of the portfolios are within one basis point of the target yield. For long holding periods, $13 \%$ to $28 \%$ of the portfolios are within one basis point of the target when the forward rate volatilities are relatively low, and less than $3 \%$ of the portfolios are within one basis point of the target yield when the volatilities are high.

Bullet portfolios have the closest returns to the target but barbell portfolios also have returns that are less than one basis point away from the target yield for short holding periods. For long holding periods, we observe unsuccessful bullet and barbell portfolios. In this respect, we cannot favor bullet or barbell portfolios over random portfolios for medium to long holding periods. Both bullet and barbell portfolios are preferable to random portfolios for short holding periods.

Table 1 Panel C shows that under the exponential decay volatility HJM framework, the exponential decay coefficient has a negative value for all yield curves considered in the study. 
This finding is consistent with that of Amin and Morton (1994), who find that even though forward rate volatilities reduce slightly from short term to medium term, these volatilities increase significantly from medium to long term. This generally leads to a negative volatility reduction coefficient. In this respect, the volatility is low at short-term and medium-term segments of the yield curve and high at the long end of the yield curve. For short to medium holding periods, the volatilities of forward rates are between 1.5\% and 1.8\%. For long holding periods, the forward rate volatilities increase up to $2.2 \%$. This could be a reason for the good performance of Macaulay's duration for short to medium holding periods and the deterioration of the performance for long holding periods. When the volatility is high, the performance of Macaulay's duration deteriorates.

When there is low volatility, the shifts in the yield curve will be small. Therefore, it is reasonable to assume a linear relation between the bond price and the yield change. In these cases, a duration matching strategy provides good immunization. When the volatility is high, however, the shifts in the yield curve are non-infinitesimal, and we have to consider the nonlinear relation between the bond price and the yield change. Under these circumstances, a duration and convexity matching strategy may provide better immunization than a duration matching strategy alone.

The success of bullet portfolios for short holding periods is expected. As mentioned in Fuller and Settle (1994), for short holding periods, the main determinant of the duration measure is time to maturity. Bullet portfolios have the closest durations to the target portfolios, and therefore, they provide the closest returns to the target yield in the short run when a duration matching strategy is used.

The performance of Fisher-Weil duration is given in Table 9 Panel B. The results on Fisher Weil duration are almost identical to those for Macaulay's duration under the exponential volatility HJM framework. Under the active portfolio management, the frequency of successful portfolios is above $96 \%$ for short to medium holding periods. For long holding periods, the performance of Fisher-Weil duration deteriorates significantly, especially under increasing and humped initial yield curves where forward rate volatilities are high. More than 
$85 \%$ of the portfolios have returns within 100 basis points of the target yield. The percentage of portfolios that have returns within one basis point of the target is less than $23 \%$.

Bullet portfolios again have the closest returns to the target and barbell portfolios have returns within one basis point neighborhood of the target yield. We observe unsuccessful bullet and barbell portfolios for long holding periods.

Since the results for Fisher-Weil duration are similar to those for Macaulay's duration, we can say that at the parts of the yield curve where forward rate volatilities are low, both of these duration measures provide successful immunization. When volatilities are high, however, traditional duration measures are not successful in immunization. This may be due to large shifts in the yield curve that cannot be hedged by only a duration matching strategy. A duration and convexity matching strategy may provide better immunization in these circumstances, since it considers the nonlinear relation between the bond price and the yield change.

Table 9 Panel $\mathrm{C}$ shows that, for short to medium holding periods, under the active portfolio management criterion, more than $96 \%$ of the portfolios are successfully immunized for short to medium holding periods. The difference between the immunization performance of the traditional risk measures and the HJM risk measure is significant for long holding periods, especially when forward rate volatilities are high. More than $81 \%$ of the portfolios are successfully immunized with the HJM duration measure. Compared to the traditional risk measures, the HJM duration measure results in at least 50\% more successfully immunized portfolios for long holding periods when forward rate volatilities are high. In this respect, one can suggest that traditional duration measures significantly underperfrom their HJM counterparts. Nevertheless, one should consider that under high volatility and long holding periods, adjusting for convexity and using a duration and convexity matching strategy might be more appropriate. If we observe similar differences in the performances of the traditional and HJM risk measures with the duration and convexity matching strategy, this will suggest that traditional risk measures are poor risk measures compared to the HJM ones.

The performance of the HJM duration measure is similar to those of the traditional risk measures under passive portfolio management. More than $95 \%$ of the portfolios have returns 
that are within 100 basis point of the target, whereas less than $22 \%$ have returns within one basis point of the target. Therefore, under passive portfolio management, traditional duration measures perform as well as their HJM counterparts under exponential decay volatility HJM framework.

Bullet portfolios produce the closest returns to the target, and both bullet and barbell portfolios are within one basis point distance of the target yield for short holding periods. The returns of bullet portfolios are within one basis point of the target for medium holding periods as well. In this respect, bullet and barbell portfolio can be preferred for short holding periods, and bullet portfolios can be preferred for medium holding periods as well. In the long run, however, we observe unsuccessful bullet and barbell portfolios and, therefore, cannot favor one portfolio formation strategy over another for long holding periods.

The HJM risk measures are our benchmark. Therefore we expect to observe better immunization performance with these measures. Nevertheless, we should realize that for short to medium holding periods, where the forward rate volatilities are low, the performance of the HJM duration measure is similar to the performances of the traditional counterparts. Therefore, if we are dealing with the low volatility part of the yield curve, traditional duration measures are easy to use alternatives that should result in successful immunization. The improved performance with the HJM duration measure is apparent when the volatilities are high over long holding period. In these cases, the HJM duration measure leads to at least 50\% more successfully immunized portfolios than traditional duration measures.

This result may suggest that we should prefer HJM measures to traditional ones. One should be careful about this conclusion in the sense that when we use benchmark HJM duration measures, we adjust for the correct volatility function. We are not able to do so with traditional measures. Therefore, when there is high volatility, traditional duration measures underperform their HJM counterparts significantly. When volatilities are high, the shifts in the yield curve will be large. Therefore, a duration matching strategy may not be a good immunization strategy for the high volatility parts of the yield curve. In these cases, a duration and convexity matching strategy may be a better alternative. We cannot favor one measure over another before we consider alternative immunization strategies. 
Table 10 shows the performance of bullet and barbell portfolios under an exponential decay volatility HJM framework. The results given in Table 10 are similar to the ones found under the constant volatility HJM framework. Bullet and barbell portfolios produce closer returns to the target for short holding periods, but their performances deteriorate as the holding period increases.

\subsection{Constant Decay Volatility One-Factor HJM Framework}

Table 11 presents the results on the performances of duration matching portfolios under the constant decay volatility HJM framework. The performances of the portfolios that are duration matched with Macaulay's, Fisher-Weil, and the constant decay one-factor HJM duration are presented in Panels A, B, and C, respectively.

The performance of Macaulay's duration under the constant decay volatility HJM framework is presented in Table 11 Panel A. The portfolios that are duration matched with Macaulay's duration perform better for long holding periods. As can be observed from Table 1 Panel B, under constant decay volatility, long holding periods correspond to lower forward rate volatilities, since forward rate volatility decreases with time to maturity. In this respect, Macaulay's duration is successful for long holding periods where the volatility is relatively low. For short holding periods, however, less than $46 \%$ of the portfolios are successfully immunized according to the active portfolio management criterion. According to passive portfolio management, more than $97 \%$ of the portfolios have returns within 100 basis point of the target. On the other hand, less than $20 \%$ of the portfolios have returns within one basis of the target.

Bullet and barbell portfolios have returns within one basis point of the target yield for short holding periods under certain cases. Therefore, for short holding periods, one might prefer a bullet or a barbell portfolio to a random portfolio. As the holding period increases, the

performance of a bullet or a barbell portfolio is not significantly different than that of any random portfolio. In this respect, for long holding periods, random portfolios provide as good as and often better immunization than bullet portfolios. 
Table 10

\section{Performance of Duration Matched Bullet and Barbell Portfolios under Exponential Decay Volatility HJM Framework}

This table shows the performance of duration matched bullet and barbell portfolios under exponential decay volatility HJM framework. Bullet and barbell portfolios are duration matched using Macaulay's, Fisher-Weil, and one-factor exponential decay volatility HJM duration measures. Panels A and B show the results on the performance of duration matched bullet and barbell portfolios, respectively. The results are analyzed according to the initial yield curves and holding periods considered. Initial yield curves considered in the study are flat, increasing, decreasing, and humped yield curves. Holding periods are one, five and ten years. For each portfolio matched with a duration measure, the first row shows the holding period return. Standard errors of the returns are in parenthesis. The following two rows are the maximum absolute (MaxAD) and relative (MaxRD) deviations of the portfolio returns from the target return.

\begin{tabular}{|c|c|c|c|c|c|c|c|c|c|c|c|c|}
\hline \multicolumn{13}{|c|}{ Panel A: Bullet Portfolios } \\
\hline & \multicolumn{3}{|c|}{$\begin{array}{c}\text { Flat } \\
\text { (August, 1989) }\end{array}$} & \multicolumn{3}{|c|}{$\begin{array}{c}\text { Increasing } \\
\text { (January, 1985) }\end{array}$} & \multicolumn{3}{|c|}{$\begin{array}{c}\text { Decreasing } \\
\text { (May, 1981) }\end{array}$} & \multicolumn{3}{|c|}{$\begin{array}{c}\text { Humped } \\
\text { (October, 1986) }\end{array}$} \\
\hline & 1 year & 5 year & 10 year & 1 year & 5 year & 10 year & 1 year & 5 year & 10 year & 1 year & 5 year & 10 year \\
\hline \multirow[t]{2}{*}{ Macaulay } & $8.1523 \%$ & $8.1558 \%$ & $8.1010 \%$ & $8.8666 \%$ & $10.7568 \%$ & $10.9185 \%$ & $14.7832 \%$ & $13.3548 \%$ & $12.5884 \%$ & $5.6248 \%$ & $6.8869 \%$ & \\
\hline & (3.69E-06) & (7.68E-04) & (9.44E-05) & (6.40E-06) & (3.27E-04) & $(1.43 \mathrm{E}-04)$ & (3.82E-06) & (1.08E-04) & (3.19E-04) & $(5.29 \mathrm{E}-06)$ & $(3.66 \mathrm{E}-04)$ & (3.56E-03) \\
\hline MaxAD & $1.80 \mathrm{E}-07$ & $1.20 \mathrm{E}-05$ & $4.55 \mathrm{E}-04$ & $3.79 \mathrm{E}-07$ & $1.81 \mathrm{E}-04$ & $1.80 \mathrm{E}-03$ & $1.83 \mathrm{E}-07$ & $5.31 \mathrm{E}-05$ & $3.15 \mathrm{E}-03$ & $3.24 \mathrm{E}-07$ & $1.25 \mathrm{E}-04$ & $1.86 \mathrm{E}-03$ \\
\hline MaxRD & $2.20 \mathrm{E}-06$ & $1.48 \mathrm{E}-04$ & $5.58 \mathrm{E}-03$ & $4.27 \mathrm{E}-06$ & $1.68 \mathrm{E}-03$ & $1.63 \mathrm{E}-02$ & $1.24 \mathrm{E}-06$ & 4.24E-04 & 2.44 & $5.76 \mathrm{E}-06$ & $1.81 \mathrm{E}-03$ & -02 \\
\hline \multirow[t]{2}{*}{ Fisher-Weil } & $8.1523 \%$ & $8.1558 \%$ & $8.1018 \%$ & $8.8666 \%$ & $10.7547 \%$ & $10.9091 \%$ & $14.7832 \%$ & $13.3551 \%$ & $12.6895 \%$ & $5.6248 \%$ & $6.8832 \%$ & $7.8000 \%$ \\
\hline & (3.69E-06) & $(7.75 \mathrm{E}-04)$ & $(9.28 \mathrm{E}-05)$ & $(6.39 \mathrm{E}-06)$ & $(2.71 \mathrm{E}-04)$ & $(1.57 \mathrm{E}-04)$ & $(3.82 \mathrm{E}-06)$ & $(1.09 \mathrm{E}-04)$ & $(1.77 \mathrm{E}-04)$ & (5.28E-06) & $(4.90 \mathrm{E}-04)$ & (3.01E-03) \\
\hline MaxAD & $1.80 \mathrm{E}-07$ & $1.27 \mathrm{E}-05$ & 4.47E-04 & $3.78 \mathrm{E}-07$ & $1.59 \mathrm{E}-04$ & $1.90 \mathrm{E}-03$ & $1.84 \mathrm{E}-07$ & $5.66 \mathrm{E}-05$ & $2.14 \mathrm{E}-03$ & $3.24 \mathrm{E}-07$ & $1.62 \mathrm{E}-04$ & $1.78 \mathrm{E}-03$ \\
\hline MaxRD & $2.20 \mathrm{E}-06$ & $1.56 \mathrm{E}-04$ & $5.49 \mathrm{E}-03$ & $4.26 \mathrm{E}-06$ & $1.48 \mathrm{E}-03$ & $1.71 \mathrm{E}-02$ & $1.24 \mathrm{E}-06$ & $4.24 \mathrm{E}-04$ & $1.66 \mathrm{E}-02$ & $5.76 \mathrm{E}-06$ & $2.35 \mathrm{E}-03$ & $2.34 \mathrm{E}-02$ \\
\hline \multirow[t]{2}{*}{ HJM } & $8.1523 \%$ & $8.1536 \%$ & $8.1258 \%$ & $8.8666 \%$ & $10.7459 \%$ & $10.9953 \%$ & $14.7832 \%$ & $13.3463 \%$ & $14.4352 \%$ & $5.6248 \%$ & $6.8902 \%$ & $7.7855 \%$ \\
\hline & $(2.36 \mathrm{E}-06)$ & $(2.34 \mathrm{E}-04)$ & $(6.86 \mathrm{E}-05)$ & (4.08E-06) & (1.01E-04) & & & & & & $(4.31 \mathrm{E}-04)$ & $(2.63 \mathrm{E}-04)$ \\
\hline MaxAD & $7.23 \mathrm{E}-08$ & $9.56 \mathrm{E}-06$ & $2.07 \mathrm{E}-04$ & $1.61 \mathrm{E}-07$ & $7.13 \mathrm{E}-05$ & $1.04 \mathrm{E}-03$ & $1.16 \mathrm{E}-07$ & $3.16 \mathrm{E}-05$ & $1.53 \mathrm{E}-03$ & $1.21 \mathrm{E}-07$ & $9.18 \mathrm{E}-05$ & $1.64 \mathrm{E}-03$ \\
\hline MaxRD & $8.87 \mathrm{E}-07$ & $1.17 \mathrm{E}-04$ & $2.54 \mathrm{E}-03$ & $1.82 \mathrm{E}-06$ & $6.64 \mathrm{E}-04$ & $9.34 \mathrm{E}-03$ & $7.86 \mathrm{E}-07$ & $2.36 \mathrm{E}-04$ & $1.19 \mathrm{E}-02$ & $2.15 \mathrm{E}-06$ & $1.33 \mathrm{E}-03$ & $2.15 \mathrm{E}-02$ \\
\hline
\end{tabular}


Table 10-Continued

\begin{tabular}{|c|c|c|c|c|c|c|c|c|c|c|c|c|}
\hline \multicolumn{13}{|c|}{ Panel B: Barbell Portfolios } \\
\hline & \multicolumn{3}{|c|}{$\begin{array}{c}\text { Flat } \\
\text { (August, 1989) } \\
\end{array}$} & \multicolumn{3}{|c|}{$\begin{array}{c}\text { Increasing } \\
\text { (January, 1985) }\end{array}$} & \multicolumn{3}{|c|}{$\begin{array}{c}\text { Decreasing } \\
(\text { May, 1981) }\end{array}$} & \multicolumn{3}{|c|}{$\begin{array}{c}\text { Humped } \\
\text { (October, 1986) } \\
\end{array}$} \\
\hline & 1 year & 5 year & 10 year & 1 year & 5 year & 10 year & 1 year & 5 year & 10 year & 1 year & 5 year & 10 year \\
\hline \multirow[t]{2}{*}{ Macaulay } & $8.1519 \%$ & $8.1407 \%$ & $8.0853 \%$ & $8.8658 \%$ & $10.7106 \%$ & $10.9688 \%$ & $14.7829 \%$ & $13.3388 \%$ & $12.8588 \%$ & $5.6239 \%$ & $6.8718 \%$ & $7.4937 \%$ \\
\hline & (8.78E-07) & (1.91E-05) & $(7.96 \mathrm{E}-05)$ & $(1.31 \mathrm{E}-06)$ & $(2.96 \mathrm{E}-05)$ & (1.24E-04) & $(6.60 \mathrm{E}-07)$ & $(7.03 \mathrm{E}-06)$ & $(2.33 \mathrm{E}-05)$ & $(1.40 \mathrm{E}-06)$ & $(3.24 \mathrm{E}-05)$ & $(1.4$ \\
\hline MaxAD & 4.49E-06 & 1.39E-04 & $6.12 \mathrm{E}-04$ & 8.37E-06 & $2.82 \mathrm{E}-04$ & $1.30 \mathrm{E}-03$ & $3.07 \mathrm{E}-06$ & $1.07 \mathrm{E}-04$ & 4.49E-04 & 8.94E-06 & $2.75 \mathrm{E}-04$ & $1.28 \mathrm{E}-03$ \\
\hline MaxRD & $5.50 \mathrm{E}-05$ & $1.70 \mathrm{E}-03$ & $7.52 \mathrm{E}-03$ & $9.44 \mathrm{E}-05$ & $2.63 \mathrm{E}-03$ & $1.17 \mathrm{E}-02$ & $2.08 \mathrm{E}-05$ & $8.00 \mathrm{E}-04$ & $3.48 \mathrm{E}-03$ & $1.59 \mathrm{E}-04$ & 3.99E-03 & $1.68 \mathrm{E}-02$ \\
\hline \multirow[t]{2}{*}{ Fisher-Weil } & $8.1519 \%$ & $8.1408 \%$ & $8.0863 \%$ & $8.8658 \%$ & $10.7105 \%$ & $10.9665 \%$ & $14.7829 \%$ & $13.3380 \%$ & $12.8526 \%$ & $5.6240 \%$ & $6.8730 \%$ & $2 \%$ \\
\hline & (8.72E-07) & $(1.88 \mathrm{E}-05)$ & $(7.73 \mathrm{E}-05)$ & $(1.31 \mathrm{E}-06)$ & (2.97E-06) & (1.27E-04) & $(6.76 \mathrm{E}-07)$ & $(8.71 \mathrm{E}-06)$ & $(3.43 \mathrm{E}-05)$ & $(1.35 \mathrm{E}-06)$ & $(3.03 \mathrm{E}-05)$ & (1.28E-04) \\
\hline MaxAD & $4.46 \mathrm{E}-06$ & $1.38 \mathrm{E}-04$ & $6.02 \mathrm{E}-04$ & $8.36 \mathrm{E}-06$ & $2.83 \mathrm{E}-04$ & $1.33 \mathrm{E}-03$ & $3.21 \mathrm{E}-06$ & $1.14 \mathrm{E}-04$ & $5.10 \mathrm{E}-04$ & $8.67 \mathrm{E}-06$ & $2.64 \mathrm{E}-04$ & $1.20 \mathrm{E}-03$ \\
\hline MaxRD & $5.47 \mathrm{E}-05$ & $1.69 \mathrm{E}-03$ & 7.39E-03 & $9.42 \mathrm{E}-05$ & $2.63 \mathrm{E}-03$ & 1.19E-02 & $2.17 \mathrm{E}-05$ & $8.57 \mathrm{E}-04$ & $3.96 \mathrm{E}-03$ & $1.54 \mathrm{E}-04$ & $3.83 \mathrm{E}-03$ & $1.58 \mathrm{E}-02$ \\
\hline \multirow[t]{2}{*}{ HJM } & $8.1521 \%$ & $8.1481 \%$ & $8.1226 \%$ & $8.8663 \%$ & $10.7254 \%$ & $11.0465 \%$ & $14.7830 \%$ & $13.3412 \%$ & $12.8699 \%$ & $5.6245 \%$ & $6.8882 \%$ & $7.5794 \%$ \\
\hline & $(4.85 \mathrm{E}-07)$ & $(2.59 \mathrm{E}-06)$ & $(3.47 \mathrm{E}-06)$ & (7.63E-07) & $(4.23 \mathrm{E}-06)$ & $(5.76 \mathrm{E}-06)$ & $(6.22 \mathrm{E}-07)$ & $(3.47 \mathrm{E}-06)$ & $(4.85 \mathrm{E}-06)$ & $(6.43 \mathrm{E}-06)$ & $(3.44 \mathrm{E}-06)$ & (4.70E-06) \\
\hline MaxAD & $2.10 \mathrm{E}-06$ & $6.48 \mathrm{E}-05$ & $2.39 \mathrm{E}-04$ & $4.01 \mathrm{E}-06$ & $1.33 \mathrm{E}-04$ & $5.25 \mathrm{E}-04$ & $2.32 \mathrm{E}-06$ & $8.23 \mathrm{E}-05$ & $3.37 \mathrm{E}-04$ & $3.70 \mathrm{E}-06$ & $1.11 \mathrm{E}-04$ & $4.23 \mathrm{E}-04$ \\
\hline MaxRD & $2.58 \mathrm{E}-05$ & $7.95 \mathrm{E}-04$ & $2.94 \mathrm{E}-03$ & $4.53 \mathrm{E}-05$ & $1.24 \mathrm{E}-03$ & 4.73E-03 & $1.57 \mathrm{E}-05$ & $6.16 \mathrm{E}-04$ & $2.61 \mathrm{E}-03$ & $6.57 \mathrm{E}-05$ & $1.62 \mathrm{E}-03$ & $5.55 \mathrm{E}-03$ \\
\hline
\end{tabular}




\section{Table 11}

\section{Performance of Duration Matched Portfolios under Constant Decay Volatility HJM Framework}

The performances of 102 portfolios are summarized. Two of the portfolios are bullet and barbell, and the rest are random portfolios. The benchmark portfolios are the ones that are matched according to the duration measure of the one factor HJM model with constant decay volatility. The other two duration measures are the Macaulay's and the Fisher-Weil durations. Panel A, B, and C presents the results the performances of immunization portfolios matched by Macaulay's duration, Fisher-Weil duration, and the constant decay volatility one factor HJM duration, respectively. The results are analyzed according to the initial yield curves and holding periods. Four initial yield curves are considered: Flat (August 1989); increasing (January 1985); decreasing (May 1981); and humped (October 1986). The three holding periods are one-, five-, and ten-years. Panel A presents the results on the performances of immunization portfolios matched by Macaulay's duration. Panel B gives the results on the performances of immunization portfolios matched by Fisher-Weil duration. Three criteria are used to assess the immunization performances of the portfolios. According to one criterion, a portfolio is considered successful in immunization if the holding period return of the portfolio is greater than or equal to the yield of the target zero coupon bond minus 5 basis points. In Panels A and B, "\% Successful” (“\%Unsuccessful”) gives the percentage of the portfolios that are successful (unsuccessful) in immunization according to the first criterion. The other two criteria assess the immunization performances by measuring the percentage of portfolios with returns within one and 100 basis points of the target yield. These are given in the next two rows. The maximum and minimum absolute (MaxAD and MinAD) and relative (MaxRD and MinRD) deviations from the target return are presented next. For each deviation, the returns of these portfolios, and the time to maturity of the bonds in the portfolios are given. Standard errors of the returns are in parentheses. Times to maturity are given in months, (T1 and T2).

\begin{tabular}{|c|c|c|c|c|c|c|c|c|c|c|c|c|}
\hline \multicolumn{13}{|c|}{ Panel A: Macaulay's Duration } \\
\hline & \multicolumn{3}{|c|}{$\begin{array}{c}\text { Flat } \\
\text { (August, 1989) }\end{array}$} & \multicolumn{3}{|c|}{$\begin{array}{c}\text { Increasing } \\
\text { (January, 1985) }\end{array}$} & \multicolumn{3}{|c|}{$\begin{array}{l}\text { Decreasing } \\
(\text { May, 1981) }\end{array}$} & \multicolumn{3}{|c|}{$\begin{array}{c}\text { Humped } \\
\text { (October, 1986) }\end{array}$} \\
\hline & 1 year & 5 year & 10 year & 1 year & 5 year & 10 year & 1 year & 5 year & 10 year & 1 year & 5 year & 10 year \\
\hline \%Successful & $40.20 \%$ & $75.49 \%$ & $91.18 \%$ & $32.35 \%$ & $62.75 \%$ & $81.37 \%$ & $45.10 \%$ & $75.49 \%$ & $82.35 \%$ & $31.37 \%$ & $59.80 \%$ & $78.43 \%$ \\
\hline Within 100 basis points & $100.00 \%$ & $100.00 \%$ & $100.00 \%$ & $97.06 \%$ & $100.00 \%$ & $100.00 \%$ & $99.02 \%$ & $100.00 \%$ & 0.990196 & $99.02 \%$ & $99.02 \%$ & $100.00 \%$ \\
\hline Within 1 basis point & $17.65 \%{ }^{* * *}$ & $9.80 \%$ & $15.69 \%$ & $10.78 \%$ & $2.94 \%$ & $2.94 \%$ & $19.61 \%{ }^{* * *}$ & $14.71 \%$ & $15.69 \%$ & $10.78 \%$ & $4.90 \%$ & $9.80 \%$ \\
\hline MaxAD & $.98 \mathrm{E}-03$ & $3.27 \mathrm{E}-03$ & $5.45 \mathrm{E}-03$ & $6.16 \mathrm{E}-02$ & 4.93E-03 & $3.85 \mathrm{E}-03$ & $1.46 \mathrm{E}-02$ & $1.67 \mathrm{E}-03$ & 03 & $1.17 \mathrm{E}-02$ & $1.24 \mathrm{E}-02$ & 4.4 \\
\hline MaxRD & $6.11 \mathrm{E}-02$ & $4.01 \mathrm{E}-02$ & $6.69 \mathrm{E}-02$ & $6.94 \mathrm{E}-01$ & 4.59E-02 & $3.47 \mathrm{E}-02$ & $9.85 \mathrm{E}-02$ & $1.25 \mathrm{E}-02$ & $3.82 \mathrm{E}-02$ & $2.08 \mathrm{E}-01$ & $1.80 \mathrm{E}-01$ & $5.78 \mathrm{E}-02$ \\
\hline Return & $\begin{array}{l}8.6507 \% \\
(9.66 \mathrm{E}-03)\end{array}$ & $\begin{array}{l}8.4814 \% \\
(9.01 \mathrm{E}-03)\end{array}$ & $\begin{array}{l}8.6913 \% \\
(5.05 \mathrm{E}-03)\end{array}$ & $\begin{array}{l}2.7100 \% \\
(1.06 \mathrm{E}-03)\end{array}$ & $\begin{array}{l}11.2321 \% \\
(3.17 \mathrm{E}-03)\end{array}$ & $\begin{array}{l}11.4837 \% \\
(4.94 \mathrm{E}-03)\end{array}$ & $\begin{array}{l}16.2398 \% \\
(4.29 \mathrm{E}-03)\end{array}$ & $\begin{array}{l}13.1823 \% \\
(2.06 \mathrm{E}-03)\end{array}$ & $\begin{array}{l}13.3970 \% \\
(6.05 \mathrm{E}-03)\end{array}$ & $\begin{array}{c}6.7929 \% \\
(1.59 \mathrm{E}-03)\end{array}$ & $\begin{array}{l}8.1427 \% \\
(1.37 \mathrm{E}-03)\end{array}$ & $\begin{array}{l}8.0626 \% \\
(4.42 \mathrm{E}-03)\end{array}$ \\
\hline $\mathrm{T} 1, \mathrm{~T} 2$ & 266,285 & 215,219 & 292,305 & 193,198 & 309,340 & 279,293 & 193,198 & 284,315 & 320,346 & 266,279 & 280,284 & 294,297 \\
\hline MinAD & $3.05 \mathrm{E}-06$ & $1.32 \mathrm{E}-05$ & $9.61 \mathrm{E}-05$ & $1.41 \mathrm{E}-05$ & 2.39E-05 & $2.18 \mathrm{E}-04$ & $1.59 \mathrm{E}-06$ & $3.69 \mathrm{E}-06$ & $1.16 \mathrm{E}-05$ & $4.69 \mathrm{E}-06$ & $3.13 \mathrm{E}-05$ & $3.55 \mathrm{E}-05$ \\
\hline MinRD & $3.75 \mathrm{E}-05$ & $1.62 \mathrm{E}-04$ & $1.18 \mathrm{E}-03$ & $1.59 \mathrm{E}-04$ & $2.23 \mathrm{E}-04$ & $1.96 \mathrm{E}-03$ & $1.07 \mathrm{E}-05$ & 2.77E-05 & $9.00 \mathrm{E}-05$ & $8.34 \mathrm{E}-05$ & $4.53 \mathrm{E}-04$ & 4.66E-04 \\
\hline Return & $\begin{array}{l}8.1526 \% \\
(5.85 \mathrm{E}-04)\end{array}$ & $\begin{array}{l}8.1532 \% \\
(4.55 \mathrm{E}-04)\end{array}$ & $\begin{array}{l}8.1561 \% \\
(2.14 \mathrm{E}-04)\end{array}$ & $\begin{array}{l}8.8681 \% \\
(8.17 \mathrm{E}-04)\end{array}$ & $\begin{array}{l}10.7364 \% \\
(5.09 \mathrm{E}-04)\end{array}$ & $\begin{array}{l}11.0772 \% \\
(7.61 \mathrm{E}-03)\end{array}$ & $\begin{array}{l}14.7831 \% \\
(7.73 \mathrm{E}-04)\end{array}$ & $\begin{array}{l}13.3498 \% \\
(2.65 \mathrm{E}-04)\end{array}$ & $\begin{array}{l}12.9025 \% \\
(1.99 \mathrm{E}-04)\end{array}$ & $\begin{array}{l}5.6244 \% \\
(9.02 \mathrm{E}-04)\end{array}$ & $\begin{array}{c}6.8963 \% \\
(6.23 \mathrm{E}-04)\end{array}$ & $\begin{array}{c}7.6253 \% \\
(1.98 \mathrm{E}-04)\end{array}$ \\
\hline $\mathrm{T} 1, \mathrm{~T} 2$ & 20,266 & 137,198 & 273,317 & 20,266 & 111,295 & 122,126 & 22,190 & 113,229 & 273,317 & 25,171 & 129,237 & 273,317 \\
\hline
\end{tabular}

* Bullet portfolio; ** Barbell portfolio; *** Both bullet and barbell portfolios 
Table 11 - Continued

\begin{tabular}{|c|c|c|c|c|c|c|c|c|c|c|c|c|}
\hline \multicolumn{13}{|c|}{ Panel B: Fisher-Weil Duration } \\
\hline & \multicolumn{3}{|c|}{$\begin{array}{c}\text { Flat } \\
\text { (August, 1989) }\end{array}$} & \multicolumn{3}{|c|}{$\begin{array}{c}\text { Increasing } \\
\text { (January, 1985) }\end{array}$} & \multicolumn{3}{|c|}{$\begin{array}{l}\text { Decreasing } \\
(\text { May, 1981) }\end{array}$} & \multicolumn{3}{|c|}{$\begin{array}{c}\text { Humped } \\
\text { (October, 1986) }\end{array}$} \\
\hline & 1 year & 5 year & 10 year & 1 year & 5 year & 10 year & 1 year & 5 year & 10 year & 1 year & 5 year & 10 year \\
\hline$\%$ Successful & $41.18 \%$ & $81.37 \%$ & $99.02 \%$ & $30.39 \%$ & $74.51 \%$ & $97.06 \%$ & $50.00 \%$ & $96.08 \%$ & $98.04 \%$ & $30.39 \%$ & $60.78 \%$ & $94.12 \%$ \\
\hline Within 100 basis points & $100.00 \%$ & $100.00 \%$ & $100.00 \%$ & $99.02 \%$ & $99.02 \%$ & $99.02 \%$ & $100.00 \%$ & $100.00 \%$ & $100.00 \%$ & $100.00 \%$ & $100.00 \%$ & $100.00 \%$ \\
\hline Within 1 basis point & $18.63 \%{ }^{* * *}$ & $9.80 \%$ & $0.98 \%$ & $11.76 \%$ & $5.88 \%$ & $0.00 \%$ & $21.57 \%^{* * *}$ & $15.69 \%^{*}$ & $1.96 \%$ & $10.78 \%$ & $3.92 \%$ & $0.98 \%$ \\
\hline $\operatorname{MaxAD}$ & $2.80 \mathrm{E}-03$ & $2.99 \mathrm{E}-03$ & $4.75 \mathrm{E}-03$ & $7.21 \mathrm{E}-02$ & $1.02 \mathrm{E}-02$ & $5.17 \mathrm{E}-03$ & $5.30 \mathrm{E}-03$ & $2.08 \mathrm{E}-03$ & $3.47 \mathrm{E}-03$ & $9.62 \mathrm{E}-03$ & $4.46 \mathrm{E}-03$ & $1.12 \mathrm{E}-02$ \\
\hline $\operatorname{MaxRD}$ & $3.44 \mathrm{E}-02$ & $3.67 \mathrm{E}-02$ & $5.83 \mathrm{E}-02$ & $8.14 \mathrm{E}-01$ & $9.53 \mathrm{E}-02$ & 4.66E-02 & $3.58 \mathrm{E}-02$ & $1.56 \mathrm{E}-02$ & $2.69 \mathrm{E}-02$ & $1.71 \mathrm{E}-01$ & $6.46 \mathrm{E}-02$ & $1.47 \mathrm{E}-01$ \\
\hline Return & $\begin{array}{l}7.8722 \% \\
(4.39 \mathrm{E}-03)\end{array}$ & $\begin{array}{l}8.4540 \% \\
(8.60 \mathrm{E}-03)\end{array}$ & $\begin{array}{l}8.6218 \% \\
(5.39 \mathrm{E}-03)\end{array}$ & $\begin{array}{l}1.6521 \% \\
(8.54 \mathrm{E}-03)\end{array}$ & $\begin{array}{l}11.7618 \% \\
(1.09 \mathrm{E}-03)\end{array}$ & $\begin{array}{l}11.6162 \% \\
(2.67 \mathrm{E}-03)\end{array}$ & $\begin{array}{l}14.2534 \% \\
(1.38 \mathrm{E}-03)\end{array}$ & $\begin{array}{l}13.1413 \% \\
(4.03 \mathrm{E}-03)\end{array}$ & $\begin{array}{l}13.2507 \% \\
(2.21 \mathrm{E}-03)\end{array}$ & $\begin{array}{c}6.5870 \% \\
(1.73 \mathrm{E}-03)\end{array}$ & $\begin{array}{c}7.3451 \% \\
(7.68 \mathrm{E}-03)\end{array}$ & $\begin{array}{l}8.7438 \% \\
(1.42 \mathrm{E}-03)\end{array}$ \\
\hline $\mathrm{T} 1, \mathrm{~T} 2$ & 247,268 & 215,219 & 297,322 & 288,345 & 240,277 & 177,190 & 196,233 & 240,277 & 245,270 & 266,285 & 215,219 & 292,301 \\
\hline MinAD & $3.01 \mathrm{E}-06$ & $6.44 \mathrm{E}-07$ & $1.02 \mathrm{E}-04$ & $7.36 \mathrm{E}-06$ & 5.29E-07 & $3.74 \mathrm{E}-04$ & $1.04 \mathrm{E}-06$ & 4.30E-06 & $3.70 \mathrm{E}-05$ & $1.50 \mathrm{E}-05$ & $3.97 \mathrm{E}-05$ & $5.61 \mathrm{E}-04$ \\
\hline MinRD & $3.69 \mathrm{E}-05$ & 7.89E-06 & $1.25 \mathrm{E}-03$ & $8.30 \mathrm{E}-05$ & $4.92 \mathrm{E}-06$ & $4.60 \mathrm{E}-03$ & 7.06E-06 & $3.22 \mathrm{E}-05$ & $2.87 \mathrm{E}-04$ & $2.66 \mathrm{E}-04$ & $5.76 \mathrm{E}-04$ & $7.35 \mathrm{E}-03$ \\
\hline Return & $\begin{array}{l}8.1526 \% \\
(5.85 \mathrm{E}-04)\end{array}$ & $\begin{array}{l}8.1546 \% \\
(4.03 \mathrm{E}-04)\end{array}$ & $\begin{array}{l}8.1567 \% \\
(1.58 \mathrm{E}-04)\end{array}$ & $\begin{array}{l}8.8659 \% \\
(3.85 \mathrm{E}-04)\end{array}$ & $\begin{array}{l}10.7387 \% \\
(5.41 \mathrm{E}-04)\end{array}$ & $\begin{array}{l}8.1840 \% \\
(1.84 \mathrm{E}-04)\end{array}$ & $\begin{array}{l}14.7834 \% \\
(7.72 \mathrm{E}-04)\end{array}$ & $\begin{array}{l}13.3499 \% \\
(3.52 \mathrm{E}-04)\end{array}$ & $\begin{array}{l}12.9073 \% \\
(3.11 \mathrm{E}-04)\end{array}$ & $\begin{array}{l}5.6263 \% \\
(8.11 \mathrm{E}-04)\end{array}$ & $\begin{array}{c}6.9034 \% \\
(5.29 \mathrm{E}-04)\end{array}$ & $\begin{array}{c}7.6778 \% \\
(4.71 \mathrm{E}-04)\end{array}$ \\
\hline $\mathrm{T} 1, \mathrm{~T} 2$ & 20,266 & 111,295 & 292,305 & 21,295 & 119,307 & $342,348^{*}$ & 22,190 & 128,200 & $324,330^{*}$ & 20,266 & 111,295 & 317,342 \\
\hline
\end{tabular}

* Bullet portfolio

** Barbell portfolio

*** Both bullet and barbell portfolios 
Table 11 - Continued

\begin{tabular}{|c|c|c|c|c|c|c|c|c|c|c|c|c|}
\hline \multicolumn{13}{|c|}{ Panel C: HJM Constant Decay Duration } \\
\hline & \multicolumn{3}{|c|}{$\begin{array}{c}\text { Flat } \\
\text { (August, 1989) }\end{array}$} & \multicolumn{3}{|c|}{$\begin{array}{c}\text { Increasing } \\
\text { (January, 1985) } \\
\end{array}$} & \multicolumn{3}{|c|}{$\begin{array}{c}\text { Decreasing } \\
\text { (May, 1981) }\end{array}$} & \multicolumn{3}{|c|}{$\begin{array}{c}\text { Humped } \\
\text { (October, 1986) }\end{array}$} \\
\hline & 1 year & 5 year & 10 year & 1 year & 5 year & 10 year & 1 year & 5 year & 10 year & 1 year & 5 year & 10 year \\
\hline \%Successful & $95.10 \%$ & $93.14 \%$ & $100.00 \%$ & $88.24 \%$ & $90.20 \%$ & $99.02 \%$ & $89.22 \%$ & $93.14 \%$ & $99.02 \%$ & $95.10 \%$ & $93.14 \%$ & $98.04 \%$ \\
\hline Within 100 basis points & $99.02 \%$ & $99.02 \%$ & $100.00 \%$ & $85.29 \%$ & $99.02 \%$ & $100.00 \%$ & $98.04 \%$ & $98.04 \%$ & $100.00 \%$ & $96.08 \%$ & $98.04 \%$ & $99.02 \%$ \\
\hline Within 1 basis point & $2.94 \%{ }^{* * *}$ & $16.67 \%$ & $0.00 \%$ & $3.92 \%^{* * *}$ & $8.82 \%$ & $0.00 \%$ & $5.88 \%{ }^{* * *}$ & $23.53 \%$ & $0.98 \%$ & $2.94 \%{ }^{* * *}$ & $7.84 \%$ & $0.00 \%$ \\
\hline MaxAD & $1.47 \mathrm{E}-02$ & $2.97 \mathrm{E}-02$ & $1.59 \mathrm{E}-03$ & $3.98 \mathrm{E}-02$ & $1.30 \mathrm{E}-02$ & $6.80 \mathrm{E}-03$ & $2.17 \mathrm{E}-02$ & $1.48 \mathrm{E}-02$ & $1.31 \mathrm{E}-02$ & $3.30 \mathrm{E}-02$ & $1.93 \mathrm{E}-02$ & $4.05 \mathrm{E}-03$ \\
\hline MaxRD & $1.80 \mathrm{E}-01$ & $3.64 \mathrm{E}-01$ & $1.95 \mathrm{E}-02$ & $4.48 \mathrm{E}-01$ & $1.21 \mathrm{E}-01$ & $6.12 \mathrm{E}-02$ & $1.47 \mathrm{E}-01$ & $1.11 \mathrm{E}-01$ & $1.02 \mathrm{E}-01$ & $5.87 \mathrm{E}-01$ & $2.30 \mathrm{E}-02$ & $5.32 \mathrm{E}-02$ \\
\hline \multirow[t]{2}{*}{ Return } & $6.6831 \%$ & $11.1269 \%$ & $8.3052 \%$ & $4.8912 \%$ & $9.4350 \%$ & $11.7787 \%$ & $16.9572 \%$ & $11.8729 \%$ & $11.5892 \%$ & $2.3245 \%$ & $8.8283 \%$ & $8.0268 \%$ \\
\hline & $(6.11 \mathrm{E}-03)$ & $(1.24 \mathrm{E}-03)$ & (8.36E-04) & (1.01E-03) & $(2.64 \mathrm{E}-03)$ & $(3.36 \mathrm{E}-03)$ & (2.74E-03) & $(2.48 \mathrm{E}-03)$ & (1.92E-03) & (1.16E-03) & (1.52E-03) & (1.66E-03) \\
\hline $\mathrm{T} 1, \mathrm{~T} 2$ & 288,345 & 277,294 & 273,317 & 159,216 & 241,287 & 122,126 & 146,215 & 248,317 & 273,317 & 238,303 & 241,302 & 273,317 \\
\hline $\operatorname{Min} A D$ & $3.23 \mathrm{E}-06$ & $1.23 \mathrm{E}-05$ & $1.56 \mathrm{E}-05$ & $1.01 \mathrm{E}-05$ & $4.20 \mathrm{E}-06$ & $7.53 \mathrm{E}-05$ & $1.36 \mathrm{E}-07$ & $6.37 \mathrm{E}-06$ & $1.43 \mathrm{E}-05$ & $7.10 \mathrm{E}-06$ & $9.73 \mathrm{E}-06$ & $3.67 \mathrm{E}-07$ \\
\hline MinRD & $3.96 \mathrm{E}-05$ & $1.50 \mathrm{E}-04$ & $1.91 \mathrm{E}-04$ & $1.14 \mathrm{E}-04$ & $3.91 \mathrm{E}-05$ & $6.78 \mathrm{E}-04$ & $9.23 \mathrm{E}-07$ & 4.77E-05 & $1.11 \mathrm{E}-04$ & $1.26 \mathrm{E}-04$ & $1.41 \mathrm{E}-04$ & $4.82 \mathrm{E}-06$ \\
\hline \multirow{2}{*}{ Return } & $8.1520 \%$ & $8.1558 \%$ & $8.1481 \%$ & $8.8657 \%$ & $10.7392 \%$ & $11.1065 \%$ & $14.7833 \%$ & $13.3501 \%$ & $12.9051 \%$ & $5.6241 \%$ & $6.9004 \%$ & $7.6218 \%$ \\
\hline & $(6.00 \mathrm{E}-05)$ & $(7.62 \mathrm{E}-05)$ & (3.39E-05) & (1.44E-04) & $(2.16 \mathrm{E}-04)$ & $(9.68 \mathrm{E}-05)$ & $(2.64 \mathrm{E}-04)$ & $(1.62 \mathrm{E}-04)$ & $(5.60 \mathrm{E}-05)$ & $(9.58 \mathrm{E}-05)$ & $(1.06 \mathrm{E}-04)$ & (6.49E-05) \\
\hline $\mathrm{T} 1, \mathrm{~T} 2$ & $12,13^{*}$ & 69,117 & 167,316 & $12,13^{*}$ & 69,277 & 262,292 & 222,266 & 254,309 & 262,292 & $12,13^{*}$ & 69,117 & 180,283 \\
\hline
\end{tabular}

Bullet portfolio

** Barbell portfolio

*** Both bullet and barbell portfolios 
Under the constant decay volatility HJM framework, high forward rate volatilities are observed for short holding periods, and the volatilities decrease with time to maturity. For a one-year holding period, forward rate volatilities are around $40 \%$ to $57 \%$. The volatilities reduce to a range of $6.7 \%$ to $9.5 \%$ in the medium term, and to a range of $3.6 \%$ to $5.2 \%$ in the long run. In this respect, especially for short holding periods, the shifts in the yield curve are not infinitesimal. Therefore, it is highly likely that the poor performance of Macaulay's measure is due to the duration matching strategy. In these high volatility segments of the yield curve, the duration and convexity matching strategy may provide better immunization. Additionally, as observed with the previous HJM frameworks, when there is low forward rate volatility, Macaulay's duration measure provides successful immunization with the duration matching strategy.

Our previous results showed that the performance of traditional risk measures deteriorate as the holding period increases. The results we present here, however, show that traditional risk measures have good immunization performance for long holding periods but not for short holding periods. According to the constant decay volatility function, volatility decreases by holding period and the highest volatility is in the short-run. According to the constant volatility function, volatility does not change with time to maturity, whereas with exponential volatility function, due to the negative decay factor, volatility increases with time to maturity. In these respects, the performance of traditional risk measures is sensitive to the volatility level, and not too sensitive to the holding period. When there is high forward rate volatility, the performance of traditional risk measures deteriorates even for short holding periods. Also traditional risk measures perform well in the long-run if forward rate volatilities are low.

The performances of immunization portfolios that are duration matched with the Fisher-Weil measure are given in Table 11 Panel B. The performances of these portfolios are similar to those matched with the Macaulay's duration. Fisher-Weil duration performs better than Macaulay's duration for medium holding periods. Under a criterion of active portfolio management, for long holding periods, more than $98 \%$ of the portfolios are successful in immunization. On the other hand, for short holding periods, less than $55 \%$ of the portfolios 
are successfully immunized. According to passive portfolio management, more than $99 \%$ of the portfolios have returns within 100 basis points of the target yield. Less than $22 \%$ of the portfolios, however, have returns within one basis point of the target.

The results on Fisher-Weil duration support the results on Macaulay's duration. When volatility is high, which is for short and medium holding periods, duration matching with traditional measures does not provide successful immunization. There can be several reasons for this underperformance. It can be due to the under-performance of these traditional risk measures. It can also be due to the immunization strategy. When there is high volatility, we should take into consideration large shifts in the yield curve, and therefore, duration and convexity matching strategy may be a better alternative. With this alternative, underperformance of the traditional measures may not be there.

Table 11 Panel $\mathrm{C}$ shows that performances of the portfolios matched by the constant decay volatility HJM duration are superior to those matched by the traditional counterparts only for short to medium holding periods. This result is expected in the sense that these holding periods correspond to the segments of the yield curve where forward rate volatilities are very high. When we use the benchmark HJM duration measures, we know the exact volatility function. Therefore, the immunization performance of HJM measures is expected to be better than the traditional ones, especially when there is high volatility. Under a criterion of active portfolio management, for short to medium holding periods, compared to the traditional measures, the HJM duration measure produces up to $65 \%$ more successfully immunized portfolios. Again one should be careful before suggesting the use of HJM risk measures over traditional ones. At the segments of the yield curve with high volatility, a duration matching strategy may not be a good immunization strategy and the under-performance of the traditional measures may be mainly due to this factor. Under a criterion of passive portfolio management, the performance of the HJM duration measure is very similar to those of the traditional duration measures. More than $98 \%$ of the portfolios have returns within 100 basis points of the target whereas less than $24 \%$ of the portfolios have returns within one basis of the target. In this respect, under passive portfolio management, traditional duration measures 
perform as well as the HJM duration measure under a constant decay volatility HJM framework.

As can be observed from Table 11 Panel $\mathrm{C}$ and Table 12, when the HJM duration measure is used, both bullet and barbell portfolios produce holding period returns within one basis point of the target for short holding periods. Hence a risk manager can prefer to use bullet portfolios for short holding periods. In medium holding periods, all bullet and barbell portfolios are successful but so are most of the random portfolios. In long holding periods, where more than $78 \%$ of the random portfolios are successful, we observe unsuccessful bullet and barbell portfolios. In this respect, for long holding periods, random portfolios perform as well as and at times better than bullet and barbell portfolios.

Table 12 shows that for short holding periods, bullet and barbell portfolios have closer returns to the target. Unlike the exponential decay volatility and constant volatility HJM frameworks, here the performances of the bullet and barbell portfolios do not deteriorate significantly as the holding period increases. The reason is due to the reduction in volatility as time to maturity increases. Unlike the exponential decay and constant volatility frameworks, with constant decay volatility, the volatilities are highest at the short-run. Nevertheless, we still observe that bullet portfolios provide successful immunization. These results suggest that the immunization performance of bullet portfolios is very good for short holding periods irrespective of the volatility level. Therefore asset-liability managers can prefer bullet portfolios to alternative portfolio formation strategies for short holding periods.

These results on the performances of duration-matched portfolios under the constant decay volatility HJM framework suggest that traditional duration measures are as successful as their HJM counterparts when forward rate volatilities are low. When these volatilities are high, under an active portfolio management criterion, the HJM duration measure leads up to $65 \%$ more successfully immunized portfolios than do the traditional duration measures. On the other hand, under a passive portfolio management criterion, traditional and HJM duration measures show similar immunization performance. In this respect, these results suggest that HJM risk measures are not necessarily better determinants of interest rate risk compared to 


\section{Table 12}

\section{Performance of Duration Matched Bullet and Barbell Portfolios under Constant Decay Volatility HJM Framework}

This table shows the performance of duration matched bullet and barbell portfolios under constant decay volatility HJM framework. Bullet and barbell portfolios are duration matched using Macaulay's, Fisher-Weil, and one-factor constant decay volatility HJM duration measures. Panels A and B show the results on the performance of duration matched bullet and barbell portfolios, respectively. The results are analyzed according to the initial yield curves and holding periods considered. Initial yield curves considered in the study are flat, increasing, decreasing, and humped yield curves. Holding periods are one, five and ten years. For each portfolio matched with a duration measure, the first row shows the holding period return. Standard errors of the returns are in parenthesis. The following two rows are the maximum absolute (MaxAD) and relative (MaxRD) deviations of the portfolio returns from the target return.

\begin{tabular}{|c|c|c|c|c|c|c|c|c|c|c|c|c|}
\hline \multicolumn{13}{|c|}{ Panel A: Bullet Portfolios } \\
\hline & \multicolumn{3}{|c|}{$\begin{array}{c}\text { Flat } \\
\text { (August, 1989) }\end{array}$} & \multicolumn{3}{|c|}{$\begin{array}{c}\text { Increasing } \\
\text { (January, 1985) }\end{array}$} & \multicolumn{3}{|c|}{$\begin{array}{c}\text { Decreasing } \\
\text { (May, 1981) }\end{array}$} & \multicolumn{3}{|c|}{$\begin{array}{c}\text { Humped } \\
\text { (October, 1986) }\end{array}$} \\
\hline & 1 year & 5 year & 10 year & 1 year & 5 year & 10 year & 1 year & 5 year & 10 year & 1 year & 5 year & 10 year \\
\hline Macaulay & $\begin{array}{c}8.1596 \% \\
(5.52 \mathrm{E}-04)\end{array}$ & $\begin{array}{c}8.2086 \% \\
(6.15 \mathrm{E}-04)\end{array}$ & $\begin{array}{c}8.1221 \% \\
(2.98 \mathrm{E}-04)\end{array}$ & $\begin{array}{c}8.8831 \% \\
(1.13 \mathrm{E}-04)\end{array}$ & $\begin{array}{l}10.7690 \% \\
(6.50 \mathrm{E}-04)\end{array}$ & $\begin{array}{r}11.1108 \% \\
(7.61 \mathrm{E}-04)\end{array}$ & $\begin{array}{r}14.7886 \% \\
(5.13 \mathrm{E}-04)\end{array}$ & $\begin{array}{l}13.3651 \% \\
(2.67 \mathrm{E}-04)\end{array}$ & $\begin{array}{r}12.9779 \% \\
(4.27 \mathrm{E}-04)\end{array}$ & $\begin{array}{c}5.6395 \% \\
(8.50 \mathrm{E}-04)\end{array}$ & $\begin{array}{c}6.9896 \% \\
(7.11 \mathrm{E}-04)\end{array}$ & $\begin{array}{c}7.5690 \% \\
(3.52 \mathrm{E}-04)\end{array}$ \\
\hline MaxAD & $7.27 \mathrm{E}-05$ & 5.41E-04 & $2.45 \mathrm{E}-04$ & $1.65 \mathrm{E}-04$ & $3.02 \mathrm{E}-04$ & $2.18 \mathrm{E}-04$ & $5.32 \mathrm{E}-05$ & $1.57 \mathrm{E}-04$ & $7.43 \mathrm{E}-04$ & $1.47 \mathrm{E}-04$ & $9.02 \mathrm{E}-04$ & $5.28 \mathrm{E}-04$ \\
\hline MaxRD & $8.92 \mathrm{E}-04$ & $6.63 \mathrm{E}-03$ & $3.00 \mathrm{E}-03$ & $1.86 \mathrm{E}-03$ & $2.82 \mathrm{E}-03$ & $1.96 \mathrm{E}-03$ & $3.60 \mathrm{E}-04$ & $1.17 \mathrm{E}-03$ & $5.75 \mathrm{E}-03$ & $2.61 \mathrm{E}-03$ & $1.31 \mathrm{E}-02$ & $6.92 \mathrm{E}-03$ \\
\hline Fisher-Weil & $\begin{array}{c}8.1596 \% \\
(5.52 \mathrm{E}-04)\end{array}$ & $\begin{array}{c}8.2090 \% \\
(6.20 \mathrm{E}-04)\end{array}$ & $\begin{array}{c}8.1840 \% \\
(1.84 \mathrm{E}-04)\end{array}$ & $\begin{array}{c}8.8831 \% \\
(1.13 \mathrm{E}-04)\end{array}$ & $\begin{array}{l}10.7655 \% \\
(6.51 \mathrm{E}-04)\end{array}$ & $\begin{array}{l}11.1113 \% \\
(5.05 \mathrm{E}-04)\end{array}$ & $\begin{array}{r}14.7886 \% \\
(5.13 \mathrm{E}-04)\end{array}$ & $\begin{array}{r}13.3648 \% \\
(2.68 \mathrm{E}-04)\end{array}$ & $\begin{array}{l}12.9073 \% \\
(3.11 \mathrm{E}-04)\end{array}$ & $\begin{array}{c}5.6395 \% \\
(8.50 \mathrm{E}-04)\end{array}$ & $\begin{array}{c}6.9855 \% \\
(6.95 \mathrm{E}-04)\end{array}$ & $\begin{array}{c}7.7245 \% \\
(2.92 \mathrm{E}-04)\end{array}$ \\
\hline MaxAD & 7.27E-05 & 5.44E-04 & $3.74 \mathrm{E}-04$ & $1.65 \mathrm{E}-04$ & 2.67E-04 & $1.23 \mathrm{E}-04$ & $5.32 \mathrm{E}-05$ & $1.53 \mathrm{E}-04$ & $3.70 \mathrm{E}-05$ & $1.47 \mathrm{E}-04$ & $8.61 \mathrm{E}-04$ & $1.03 \mathrm{E}-03$ \\
\hline MaxRD & 8.92E-04 & $6.68 \mathrm{E}-03$ & 4.60E-03 & $1.86 \mathrm{E}-03$ & $2.48 \mathrm{E}-03$ & $1.11 \mathrm{E}-03$ & $3.60 \mathrm{E}-04$ & $1.15 \mathrm{E}-03$ & $2.87 \mathrm{E}-04$ & $2.61 \mathrm{E}-03$ & $1.25 \mathrm{E}-02$ & $1.35 \mathrm{E}-02$ \\
\hline HJM & $\begin{array}{c}8.1520 \% \\
(6.00 \mathrm{E}-05)\end{array}$ & $\begin{array}{c}8.2197 \% \\
(1.66 \mathrm{E}-04)\end{array}$ & $\begin{array}{c}8.1771 \% \\
(2.57 \mathrm{E}-04)\end{array}$ & $\begin{array}{c}8.8657 \% \\
(1.44 \mathrm{E}-04)\end{array}$ & $\begin{array}{l}11.0045 \% \\
(6.83 \mathrm{E}-04)\end{array}$ & $\begin{array}{r}11.1853 \% \\
(1.01 \mathrm{E}-04)\end{array}$ & $\begin{array}{r}14.7830 \% \\
(6.07 \mathrm{E}-05)\end{array}$ & $\begin{array}{r}13.4078 \% \\
(2.76 \mathrm{E}-04)\end{array}$ & $\begin{array}{l}12.8184 \% \\
(1.66 \mathrm{E}-04)\end{array}$ & $\begin{array}{c}5.6241 \% \\
(9.58 \mathrm{E}-05)\end{array}$ & $\begin{array}{c}7.0014 \% \\
(6.43 \mathrm{E}-04)\end{array}$ & $\begin{array}{c}7.6565 \% \\
(2.77 \mathrm{E}-04)\end{array}$ \\
\hline MaxAD & $3.23 \mathrm{E}-06$ & $6.51 \mathrm{E}-04$ & $3.06 \mathrm{E}-04$ & $1.01 \mathrm{E}-05$ & $2.66 \mathrm{E}-03$ & $8.64 \mathrm{E}-04$ & $2.69 \mathrm{E}-06$ & $5.83 \mathrm{E}-04$ & $8.53 \mathrm{E}-04$ & $7.10 \mathrm{E}-06$ & $1.02 \mathrm{E}-03$ & $3.48 \mathrm{E}-04$ \\
\hline MaxRD & $3.96 \mathrm{E}-05$ & $7.98 \mathrm{E}-03$ & $3.76 \mathrm{E}-03$ & $1.14 \mathrm{E}-04$ & $2.47 \mathrm{E}-02$ & $7.78 \mathrm{E}-03$ & $1.82 \mathrm{E}-05$ & 4.37E-03 & $6.61 \mathrm{E}-03$ & $1.26 \mathrm{E}-04$ & $1.48 \mathrm{E}-02$ & $4.56 \mathrm{E}-03$ \\
\hline
\end{tabular}


Table 12 - Continued

\begin{tabular}{|c|c|c|c|c|c|c|c|c|c|c|c|c|}
\hline \multicolumn{13}{|c|}{ Panel B: Barbell Portfolios } \\
\hline & \multicolumn{3}{|c|}{$\begin{array}{c}\text { Flat } \\
\text { (August, 1989) }\end{array}$} & \multicolumn{3}{|c|}{$\begin{array}{c}\text { Increasing } \\
\text { (January, 1985) }\end{array}$} & \multicolumn{3}{|c|}{$\begin{array}{c}\text { Decreasing } \\
\text { (May, 1981) }\end{array}$} & \multicolumn{3}{|c|}{$\begin{array}{c}\text { Humped } \\
\text { (October, 1986) }\end{array}$} \\
\hline & 1 year & 5 year & 10 year & 1 year & 5 year & 10 year & 1 year & 5 year & 10 year & 1 year & 5 year & 10 year \\
\hline Macaulay & $\begin{array}{c}8.1578 \% \\
(5.48 \mathrm{E}-05)\end{array}$ & $\begin{array}{c}8.2131 \% \\
(4.44 \mathrm{E}-04)\end{array}$ & $\begin{array}{c}8.2810 \% \\
(8.78 \mathrm{E}-04)\end{array}$ & $\begin{array}{c}8.8779 \% \\
(8.45 \mathrm{E}-05)\end{array}$ & $\begin{array}{r}10.8597 \% \\
(6.78 \mathrm{E}-04)\end{array}$ & $\begin{array}{r}11.3757 \% \\
(1.32 \mathrm{E}-04)\end{array}$ & $\begin{array}{l}14.7869 \% \\
(4.61 E-05)\end{array}$ & $\begin{array}{l}13.3904 \% \\
(3.78 \mathrm{E}-04)\end{array}$ & $\begin{array}{r}12.9970 \% \\
(7.38 \mathrm{E}-04)\end{array}$ & $\begin{array}{c}5.6360 \% \\
(8.09 \mathrm{E}-05)\end{array}$ & $\begin{array}{c}7.0164 \% \\
(6.41 \mathrm{E}-04)\end{array}$ & $\begin{array}{c}7.8877 \% \\
(1.25 \mathrm{E}-04)\end{array}$ \\
\hline MaxAD & $5.48 \mathrm{E}-05$ & $5.85 \mathrm{E}-04$ & $1.34 \mathrm{E}-03$ & $1.12 \mathrm{E}-04$ & $1.21 \mathrm{E}-03$ & $2.77 \mathrm{E}-03$ & $3.66 \mathrm{E}-05$ & 4.09E-04 & 9.33E-04 & $1.12 \mathrm{E}-04$ & $1.17 \mathrm{E}-03$ & $2.66 \mathrm{E}-03$ \\
\hline $\operatorname{MaxRD}$ & $6.72 \mathrm{E}-04$ & 7.17E-03 & $1.65 \mathrm{E}-02$ & $1.26 \mathrm{E}-03$ & $1.13 \mathrm{E}-02$ & $2.49 \mathrm{E}-02$ & $2.47 \mathrm{E}-04$ & $3.06 \mathrm{E}-03$ & $7.23 \mathrm{E}-03$ & $1.99 \mathrm{E}-03$ & $1.70 \mathrm{E}-02$ & $3.49 \mathrm{E}-02$ \\
\hline Fisher-Weil & $\begin{array}{c}8.1578 \% \\
(5.46 \mathrm{E}-05)\end{array}$ & $\begin{array}{c}8.2129 \% \\
(4.44 \mathrm{E}-04)\end{array}$ & $\begin{array}{c}8.2805 \% \\
(8.76 \mathrm{E}-04)\end{array}$ & $\begin{array}{c}8.8779 \% \\
(8.46 \mathrm{E}-05)\end{array}$ & $\begin{array}{r}10.8609 \% \\
(6.84 \mathrm{E}-04)\end{array}$ & $\begin{array}{r}11.3795 \% \\
(1.34 \mathrm{E}-04)\end{array}$ & $\begin{array}{l}14.7870 \% \\
(4.74 \mathrm{E}-05)\end{array}$ & $\begin{array}{l}13.3916 \% \\
(3.87 \mathrm{E}-04)\end{array}$ & $\begin{array}{r}13.0002 \% \\
(7.57 \mathrm{E}-04)\end{array}$ & $\begin{array}{c}5.6358 \% \\
(7.94 \mathrm{E}-05)\end{array}$ & $\begin{array}{c}7.0146 \% \\
(6.35 \mathrm{E}-04)\end{array}$ & $\begin{array}{c}7.8862 \% \\
(1.25 \mathrm{E}-04)\end{array}$ \\
\hline MaxAD & $5.46 \mathrm{E}-05$ & $5.83 \mathrm{E}-04$ & $1.34 \mathrm{E}-03$ & $1.12 \mathrm{E}-04$ & $1.22 \mathrm{E}-03$ & $2.81 \mathrm{E}-03$ & $3.79 \mathrm{E}-05$ & $4.22 \mathrm{E}-04$ & $9.66 \mathrm{E}-04$ & $1.09 \mathrm{E}-04$ & $1.15 \mathrm{E}-03$ & $2.64 \mathrm{E}-03$ \\
\hline $\operatorname{MaxRD}$ & $6.69 \mathrm{E}-04$ & $7.15 \mathrm{E}-03$ & $1.64 \mathrm{E}-02$ & $1.26 \mathrm{E}-03$ & $1.14 \mathrm{E}-02$ & $2.53 \mathrm{E}-02$ & $2.57 \mathrm{E}-04$ & $3.16 \mathrm{E}-03$ & $7.48 \mathrm{E}-03$ & $1.94 \mathrm{E}-03$ & $1.67 \mathrm{E}-02$ & $3.47 \mathrm{E}-02$ \\
\hline HJM & $\begin{array}{c}8.1510 \% \\
(1.16 \mathrm{E}-05)\end{array}$ & $\begin{array}{c}8.1370 \% \\
(1.93 \mathrm{E}-05)\end{array}$ & $\begin{array}{c}8.1102 \% \\
(1.80 \mathrm{E}-05)\end{array}$ & $\begin{array}{c}8.8636 \% \\
(1.99 \mathrm{E}-05)\end{array}$ & $\begin{array}{r}10.6957 \% \\
(3.57 \mathrm{E}-05)\end{array}$ & $\begin{array}{r}11.0037 \% \\
(3.35 \mathrm{E}-05)\end{array}$ & $\begin{array}{r}14.7823 \% \\
(1.04 \mathrm{E}-05)\end{array}$ & $\begin{array}{l}13.3348 \% \\
(2.07 \mathrm{E}-05)\end{array}$ & $\begin{array}{l}12.8656 \% \\
(2.29 \mathrm{E}-05)\end{array}$ & $\begin{array}{c}5.6219 \% \\
(1.74 \mathrm{E}-05)\end{array}$ & $\begin{array}{c}6.8639 \% \\
(2.85 \mathrm{E}-05)\end{array}$ & $\begin{array}{c}7.5477 \% \\
(2.64 \mathrm{E}-05)\end{array}$ \\
\hline MaxAD & $1.38 \mathrm{E}-05$ & $1.76 \mathrm{E}-04$ & $3.63 \mathrm{E}-04$ & $3.11 \mathrm{E}-05$ & $4.31 \mathrm{E}-04$ & $9.53 \mathrm{E}-04$ & $9.27 \mathrm{E}-06$ & $1.47 \mathrm{E}-04$ & $3.81 \mathrm{E}-04$ & $2.90 \mathrm{E}-05$ & $3.55 \mathrm{E}-04$ & $7.41 \mathrm{E}-04$ \\
\hline MaxRD & $1.69 \mathrm{E}-04$ & $2.16 \mathrm{E}-03$ & $4.46 \mathrm{E}-03$ & $3.51 \mathrm{E}-04$ & $4.01 \mathrm{E}-03$ & 8.59E-03 & $6.27 \mathrm{E}-05$ & $1.10 \mathrm{E}-03$ & $2.95 \mathrm{E}-03$ & $5.15 \mathrm{E}-04$ & $5.15 \mathrm{E}-03$ & $9.72 \mathrm{E}-03$ \\
\hline
\end{tabular}


their traditional counterparts. Rather, these results can be due to the reason that when there is high volatility, a duration matching strategy may not be a good immunization strategy.

\subsection{Humped Volatility One-Factor HJM Framework}

Table 13 presents the results on the performances of duration-matched portfolios under the humped volatility one factor HJM framework. Panels A, B, and C show the results on the performances of the portfolios matched by Macaulay's, Fisher Weil, and the HJM duration measure, respectively.

Table 13 Panel A shows that almost all portfolios matched by Macaulay's duration are successful for all holding periods under active portfolio management. Under passive portfolio management, $100 \%$ of the portfolios have returns within 100 basis points of the target in almost all cases. Also 30\% to $95 \%$ of the portfolios have returns within one basis point of the target. In these respects, Macaulay's duration measures can be considered to be successful in immunization according to both active and passive portfolio management.

Bullet and barbell portfolios produce returns that are within one basis point of the target yield for all holding periods except the 10-year holding period under a decreasing yield curve. For this specific case, the barbell portfolio is still within one basis point but the bullet is not. Bullet portfolios are the ones with the closest returns to the target for short holding periods. Random portfolios provide immunization as effective as bullet and barbell portfolios in almost all cases.

Table 13 Panel B shows the performance of Fisher-Weil duration. Similar to the performance of Macaulay's duration, the portfolios that are duration matched with the FisherWeil measure can be considered to be successful for all holding periods under both active and passive portfolio management. Bullet portfolios have the closest returns to the target for short holding periods. Random portfolios are as good as bullet and barbell portfolios, for medium to long holding periods.

As can be observed in Table 13 Panel C, the HJM duration measure produces results that are very close to those of their traditional counterparts under the humped volatility HJM framework. Almost all portfolios are successful for all holding periods according to both 


\section{Table 13}

\section{Performance of Duration Matched Portfolios under Humped Volatility HJM Framework}

The performances of 102 portfolios are summarized. Two of the portfolios are bullet and barbell, and the rest are random portfolios. The benchmark portfolios are the ones that are matched according to the duration measure of the one factor HJM model with humped volatility. The other two duration measures are the Macaulay's and the Fisher-Weil durations. Panel A, B, and C presents the results the performances of immunization portfolios matched by Macaulay's duration, Fisher-Weil duration, and the humped volatility one factor HJM duration, respectively. The results are analyzed according to the initial yield curves and holding periods. Four initial yield curves are considered: Flat (August 1989); increasing (January 1985); decreasing (May 1981); and humped (October 1986). The three holding periods are one-, five-, and ten-years. Panel A presents the results on the performances of immunization portfolios matched by Macaulay's duration. Panel B gives the results on the performances of immunization portfolios matched by Fisher-Weil duration. Three criteria are used to assess the immunization performances of the portfolios. According to one criterion, a portfolio is considered successful in immunization if the holding period return of the portfolio is greater than or equal to the yield of the target zero coupon bond minus 5 basis points. In Panels A and B, "\% Successful" ("\%Unsuccessful") gives the percentage of the portfolios that are successful (unsuccessful) in immunization according to the first criterion. The other two criteria assess the immunization performances by measuring the percentage of portfolios with returns within one and 100 basis points of the target yield. These are given in the next two rows. The maximum and minimum absolute (MaxAD and MinAD) and relative (MaxRD and MinRD) deviations from the target return are presented next. For each deviation, the returns of these portfolios, and the time to maturity of the bonds in the portfolios are given. Standard errors of the returns are in parentheses. Times to maturity are given in months, (T1, T2).

\begin{tabular}{|c|c|c|c|c|c|c|c|c|c|c|c|c|}
\hline \multicolumn{13}{|c|}{ Panel A: Macaulay's Duration } \\
\hline & \multicolumn{3}{|c|}{$\begin{array}{c}\text { Flat } \\
\text { (August, 1989) } \\
\end{array}$} & \multicolumn{3}{|c|}{$\begin{array}{c}\text { Increasing } \\
\text { (January, 1985) }\end{array}$} & \multicolumn{3}{|c|}{$\begin{array}{l}\text { Decreasing } \\
(\text { May, 1981) }\end{array}$} & \multicolumn{3}{|c|}{$\begin{array}{c}\text { Humped } \\
\text { (October, 1986) }\end{array}$} \\
\hline & 1 year & 5 year & 10 year & 1 year & 5 year & 10 year & 1 year & 5 year & 10 year & 1 year & 5 year & 10 year \\
\hline$\%$ Successful & $99.02 \%$ & $100.00 \%$ & $100.00 \%$ & $97.06 \%$ & $100.00 \%$ & $98.04 \%$ & $99.02 \%$ & $100.00 \%$ & $100.00 \%$ & $98.04 \%$ & $98.04 \%$ & $100.00 \%$ \\
\hline Within 100 basis points & $100.00 \%$ & $100.00 \%$ & $100.00 \%$ & $99.02 \%$ & $100.00 \%$ & $100.00 \%$ & $100.00 \%$ & $100.00 \%$ & $100.00 \%$ & $100.00 \%$ & $100.00 \%$ & $100.00 \%$ \\
\hline Within 1 basis point & $43.14 \%{ }^{* * *}$ & $52.94 \%{ }^{* * *}$ & $95.10 \%{ }^{* * *}$ & $31.37 \%{ }^{* * *}$ & $49.02 \%^{* * *}$ & $59.80 \%$ & $49.02 \%^{* * *}$ & $94.12 \%^{* * *}$ & $93.14 \%{ }^{* *}$ & $31.37 \%{ }^{* * *}$ & $47.06 \%{ }^{* * *}$ & $75.49 \%{ }^{*}$ \\
\hline MaxAD & $1.51 \mathrm{E}-03$ & $3.78 \mathrm{E}-04$ & $3.40 \mathrm{E}-04$ & $1.06 \mathrm{E}-02$ & $2.50 \mathrm{E}-03$ & $4.21 \mathrm{E}-03$ & 3.35E-03 & $4.45 \mathrm{E}-04$ & $6.95 \mathrm{E}-04$ & 2.33E-03 & $1.66 \mathrm{E}-03$ & $2.24 \mathrm{E}-04$ \\
\hline MaxRD & $1.85 \mathrm{E}-02$ & $4.63 \mathrm{E}-03$ & 4.17E-03 & $1.20 \mathrm{E}-01$ & $2.33 \mathrm{E}-02$ & $3.79 \mathrm{E}-02$ & $2.27 \mathrm{E}-02$ & 3.33E-03 & $5.39 \mathrm{E}-03$ & $4.14 \mathrm{E}-02$ & $2.40 \mathrm{E}-02$ & 2.94E-03 \\
\hline Return & $\begin{array}{l}8.0012 \% \\
(1.64 \mathrm{E}-03)\end{array}$ & $\begin{array}{l}8.1924 \% \\
(4.15 \mathrm{E}-05)\end{array}$ & $\begin{array}{l}8.1805 \% \\
(7.03 \mathrm{E}-03)\end{array}$ & $\begin{array}{c}7.8064 \% \\
(7.43 \mathrm{E}-03)\end{array}$ & $\begin{array}{l}10.9893 \% \\
(1.75 \mathrm{E}-03)\end{array}$ & $\begin{array}{l}10.6782 \% \\
(1.75 \mathrm{E}-03)\end{array}$ & $\begin{array}{l}14.4480 \% \\
(9.68 \mathrm{E}-03)\end{array}$ & $\begin{array}{l}13.3939 \% \\
(4.85 \mathrm{E}-04)\end{array}$ & $\begin{array}{l}12.9732 \% \\
(3.98 \mathrm{E}-03)\end{array}$ & $\begin{array}{l}5.3918 \% \\
(2.59 \mathrm{E}-03)\end{array}$ & $\begin{array}{c}6.7336 \% \\
(1.72 \mathrm{E}-03)\end{array}$ & $\begin{array}{c}7.5993 \% \\
(2.09 \mathrm{E}-04)\end{array}$ \\
\hline $\mathrm{T} 1, \mathrm{~T} 2$ & 266,285 & 313,345 & 292,301 & 303,339 & 309,340 & 279,293 & 193,198 & 313,345 & 292,301 & 266,279 & 280,284 & 294,297 \\
\hline MinAD & $1.61 \mathrm{E}-08$ & $1.41 \mathrm{E}-07$ & 8.87E-08 & $3.20 \mathrm{E}-08$ & $2.26 \mathrm{E}-07$ & $1.56 \mathrm{E}-06$ & $1.54 \mathrm{E}-08$ & $3.06 \mathrm{E}-07$ & $3.05 \mathrm{E}-07$ & $2.73 \mathrm{E}-08$ & $3.26 \mathrm{E}-07$ & $1.98 \mathrm{E}-06$ \\
\hline $\operatorname{MinRD}$ & $1.97 \mathrm{E}-07$ & $1.73 \mathrm{E}-06$ & $1.09 \mathrm{E}-06$ & $3.61 \mathrm{E}-07$ & $2.10 \mathrm{E}-06$ & $1.40 \mathrm{E}-05$ & $1.04 \mathrm{E}-07$ & $2.29 \mathrm{E}-06$ & $2.36 \mathrm{E}-06$ & $4.85 \mathrm{E}-07$ & 4.72E-06 & $2.59 \mathrm{E}-05$ \\
\hline \multirow[t]{2}{*}{ Return } & $8.1523 \%$ & $8.1546 \%$ & $8.1465 \%$ & $8.8667 \%$ & $10.7388 \%$ & $11.0988 \%$ & $14.7832 \%$ & $13.3495 \%$ & $12.9036 \%$ & $5.6248 \%$ & $6.8994 \%$ & $7.6215 \%$ \\
\hline & (3.81E-06) & (9.61E-06) & $(4.18 \mathrm{E}-05)$ & $(6.44 \mathrm{E}-06)$ & $(1.84 \mathrm{E}-05)$ & $(4.68 \mathrm{E}-05)$ & $(3.84 \mathrm{E}-06)$ & $(1.43 \mathrm{E}-05)$ & $(2.66 \mathrm{E}-05)$ & $(5.31 \mathrm{E}-06)$ & (1.91E-05) & (1.15E-04) \\
\hline $\mathrm{T} 1, \mathrm{~T} 2$ & $12,13^{*}$ & 71,196 & 220,258 & $12,13^{*}$ & 78,142 & 254,329 & $12,13^{*}$ & 69,277 & 205,325 & $12,13^{*}$ & 72,224 & 263,311 \\
\hline
\end{tabular}


Table 13 - Continued

\begin{tabular}{|c|c|c|c|c|c|c|c|c|c|c|c|c|}
\hline \multicolumn{13}{|c|}{ Panel B: Fisher-Weil Duration } \\
\hline & \multicolumn{3}{|c|}{$\begin{array}{c}\text { Flat } \\
\text { (August, 1989) }\end{array}$} & \multicolumn{3}{|c|}{$\begin{array}{c}\text { Increasing } \\
\text { (January, 1985) }\end{array}$} & \multicolumn{3}{|c|}{$\begin{array}{l}\text { Decreasing } \\
\text { (May, 1981) }\end{array}$} & \multicolumn{3}{|c|}{$\begin{array}{c}\text { Humped } \\
\text { (October, 1986) }\end{array}$} \\
\hline & 1 year & 5 year & 10 year & 1 year & 5 year & 10 year & 1 year & 5 year & 10 year & 1 year & 5 year & 10 year \\
\hline$\%$ Successful & $100.00 \%$ & $99.02 \%$ & $99.02 \%$ & $98.04 \%$ & $100.00 \%$ & $97.06 \%$ & $97.06 \%$ & $99.02 \%$ & $99.02 \%$ & $98.04 \%$ & $98.04 \%$ & $99.02 \%$ \\
\hline Within 100 basis points & $100.00 \%$ & $100.00 \%$ & $100.00 \%$ & $98.04 \%$ & $100.00 \%$ & $99.02 \%$ & $100.00 \%$ & $100.00 \%$ & $99.02 \%$ & $100.00 \%$ & $100.00 \%$ & $100.00 \%$ \\
\hline Within 1 basis point & $44.12 \%{ }^{* * *}$ & $52.94 \%{ }^{* * *}$ & $91.18 \%{ }^{* * *}$ & $31.37 \%{ }^{* * *}$ & $49.02 \%{ }^{* * *}$ & $49.02 \%$ & $47.06 \%{ }^{* * *}$ & $58.82 \%{ }^{* * *}$ & $94.12 \%{ }^{* *}$ & $31.37 \%{ }^{* * *}$ & $46.08 \%{ }^{* * *}$ & $70.59 \%{ }^{* * *}$ \\
\hline MaxAD & $1.38 \mathrm{E}-03$ & $9.94 \mathrm{E}-04$ & $2.74 \mathrm{E}-03$ & $3.33 \mathrm{E}-02$ & $1.19 \mathrm{E}-03$ & $1.03 \mathrm{E}-02$ & $5.83 \mathrm{E}-03$ & $1.88 \mathrm{E}-03$ & $4.30 \mathrm{E}-04$ & $5.74 \mathrm{E}-03$ & $1.15 \mathrm{E}-03$ & $1.76 \mathrm{E}-03$ \\
\hline MaxRD & $1.70 \mathrm{E}-02$ & $1.22 \mathrm{E}-02$ & $3.36 \mathrm{E}-02$ & $3.75 \mathrm{E}-01$ & $1.10 \mathrm{E}-02$ & $9.24 \mathrm{E}-02$ & $3.94 \mathrm{E}-02$ & $1.41 \mathrm{E}-02$ & $3.33 \mathrm{E}-03$ & $1.02 \mathrm{E}-01$ & $1.66 \mathrm{E}-02$ & $2.31 \mathrm{E}-02$ \\
\hline \multirow[t]{2}{*}{ Return } & $8.2906 \%$ & $8.0552 \%$ & $7.8729 \%$ & $12.1926 \%$ & $10.8574 \%$ & $10.0736 \%$ & $14.2002 \%$ & $13.1614 \%$ & $12.9466 \%$ & $5.0504 \%$ & $7.0142 \%$ & $7.7975 \%$ \\
\hline & $(6.88 \mathrm{E}-04)$ & $(3.46 \mathrm{E}-03)$ & (4.49E-03) & $(1.45 \mathrm{E}-03)$ & $(5.87 \mathrm{E}-04)$ & $(1.06 \mathrm{E}-03)$ & $(2.35 \mathrm{E}-03)$ & $(4.89 \mathrm{E}-03)$ & $(2.08 \mathrm{E}-03)$ & $(4.57 \mathrm{E}-03)$ & $(4.69 \mathrm{E}-04)$ & $(3.72 \mathrm{E}-03)$ \\
\hline $\mathrm{T} 1, \mathrm{~T} 2$ & 308,345 & 215,219 & 292,301 & 288,345 & 277,294 & $336,347^{*}$ & 288,345 & 284,315 & 297,322 & 266,285 & 313,345 & 177,190 \\
\hline $\operatorname{Min} A D$ & $1.61 \mathrm{E}-08$ & $1.38 \mathrm{E}-07$ & $2.05 \mathrm{E}-07$ & $3.19 \mathrm{E}-08$ & 4.89E-08 & $5.33 \mathrm{E}-06$ & $1.54 \mathrm{E}-08$ & $8.22 \mathrm{E}-09$ & $1.58 \mathrm{E}-06$ & $2.73 \mathrm{E}-08$ & $2.75 \mathrm{E}-08$ & $3.96 \mathrm{E}-07$ \\
\hline MinRD & $1.97 \mathrm{E}-07$ & $1.70 \mathrm{E}-06$ & $2.52 \mathrm{E}-06$ & $3.60 \mathrm{E}-07$ & $4.55 \mathrm{E}-07$ & $4.80 \mathrm{E}-05$ & $1.04 \mathrm{E}-07$ & $6.16 \mathrm{E}-08$ & $1.23 \mathrm{E}-05$ & $4.85 \mathrm{E}-07$ & $3.99 \mathrm{E}-07$ & $5.19 \mathrm{E}-06$ \\
\hline \multirow[t]{2}{*}{ Return } & $8.1523 \%$ & $8.1546 \%$ & $8.1465 \%$ & $8.8667 \%$ & $10.7388 \%$ & $11.0984 \%$ & $14.7832 \%$ & $13.3495 \%$ & $12.9038 \%$ & $5.6248 \%$ & $6.8994 \%$ & $7.6217 \%$ \\
\hline & $(3.80 \mathrm{E}-06)$ & $(9.65 \mathrm{E}-06)$ & $(3.72 \mathrm{E}-05)$ & (6.44E-06) & $(1.80 \mathrm{E}-05)$ & $(5.80 \mathrm{E}-05)$ & (8.84E-06) & $(1.45 \mathrm{E}-05)$ & $(1.78 \mathrm{E}-05)$ & $(5.31 \mathrm{E}-06)$ & $(1.87 \mathrm{E}-05)$ & $(6.04 \mathrm{E}-05)$ \\
\hline $\mathrm{T} 1, \mathrm{~T} 2$ & $12,13^{*}$ & 71,196 & 195,327 & $12,13^{*}$ & 78,142 & 269,323 & $12,13^{*}$ & 69,277 & 210,338 & $12,13^{*}$ & 72,224 & 220,330 \\
\hline
\end{tabular}

Bullet portfolio

** Barbell portfolio

*** Both bullet and barbell portfolios 
Table 13 - Continued

\begin{tabular}{|c|c|c|c|c|c|c|c|c|c|c|c|c|}
\hline \multicolumn{13}{|c|}{ Panel C: HJM Humped Duration } \\
\hline & \multicolumn{3}{|c|}{$\begin{array}{c}\text { Flat } \\
\text { (August, 1989) } \\
\end{array}$} & \multicolumn{3}{|c|}{$\begin{array}{c}\text { Increasing } \\
\text { (January, 1985) }\end{array}$} & \multicolumn{3}{|c|}{$\begin{array}{l}\text { Decreasing } \\
(\text { May, 1981) }\end{array}$} & \multicolumn{3}{|c|}{$\begin{array}{c}\text { Humped } \\
\text { (October, 1986) }\end{array}$} \\
\hline & 1 year & 5 year & 10 year & 1 year & 5 year & 10 year & 1 year & 5 year & 10 year & 1 year & 5 year & 10 year \\
\hline$\%$ Successful & $100.00 \%$ & $99.02 \%$ & $99.02 \%$ & $100.00 \%$ & $99.02 \%$ & $99.02 \%$ & $99.02 \%$ & $99.02 \%$ & $100.00 \%$ & $99.02 \%$ & $99.02 \%$ & $100.00 \%$ \\
\hline Within 100 basis points & $100.00 \%$ & $100.00 \%$ & $100.00 \%$ & $100.00 \%$ & $100.00 \%$ & $100.00 \%$ & $100.00 \%$ & $100.00 \%$ & $100.00 \%$ & $100.00 \%$ & $100.00 \%$ & $100.00 \%$ \\
\hline Within 1 basis point & $50 \%{ }^{* * *}$ & $57.84 \%{ }^{* * *}$ & $92.16 \%^{* * *}$ & $40.20 \%{ }^{* * *}$ & $53.92 \%{ }^{* * *}$ & $90.20 \%{ }^{* *}$ & $49.02 \%{ }^{* * *}$ & $63.73 \%^{* * *}$ & $94.12 \%^{* *}$ & $39.22 \%{ }^{* * *}$ & $51.96 \%{ }^{* * *}$ & $86.27 \%^{* * *}$ \\
\hline $\operatorname{MaxAD}$ & 7.24E-04 & $5.21 \mathrm{E}-04$ & $1.42 \mathrm{E}-03$ & $2.04 \mathrm{E}-03$ & $1.56 \mathrm{E}-03$ & $1.09 \mathrm{E}-03$ & $4.64 \mathrm{E}-03$ & $9.09 \mathrm{E}-04$ & 4.91E-04 & $2.31 \mathrm{E}-03$ & $3.59 \mathrm{E}-03$ & $3.74 \mathrm{E}-04$ \\
\hline MaxRD & $8.88 \mathrm{E}-03$ & $6.39 \mathrm{E}-03$ & $1.75 \mathrm{E}-02$ & $2.30 \mathrm{E}-02$ & $1.45 \mathrm{E}-02$ & $9.84 \mathrm{E}-03$ & $3.14 \mathrm{E}-02$ & $6.81 \mathrm{E}-03$ & $5.95 \mathrm{E}-04$ & 4.10E-02 & $5.20 \mathrm{E}-02$ & 4.91E-03 \\
\hline Return & $\begin{array}{l}8.2247 \% \\
(4.67 \mathrm{E}-04)\end{array}$ & $\begin{array}{c}8.1024 \% \\
(7.37 \mathrm{E}-04)\end{array}$ & $\begin{array}{l}8.0043 \% \\
(2.18 \mathrm{E}-03)\end{array}$ & $\begin{array}{c}9.0704 \% \\
(4.74 \mathrm{E}-04)\end{array}$ & $\begin{array}{l}10.8945 \% \\
(7.98 \mathrm{E}-04)\end{array}$ & $\begin{array}{l}11.2082 \% \\
(1.09 \mathrm{E}-03)\end{array}$ & $\begin{array}{l}14.3195 \% \\
(1.40 \mathrm{E}-03)\end{array}$ & $\begin{array}{l}13.2586 \% \\
(2.23 \mathrm{E}-03)\end{array}$ & $\begin{array}{l}12.9631 \% \\
(4.91 \mathrm{E}-04)\end{array}$ & $\begin{array}{l}5.8555 \% \\
(2.12 \mathrm{E}-03)\end{array}$ & $\begin{array}{c}6.5406 \% \\
(8.41 \mathrm{E}-03)\end{array}$ & $\begin{array}{r}7.6592 \% \\
(1.35 \mathrm{E}-04)\end{array}$ \\
\hline $\mathrm{T} 1, \mathrm{~T} 2$ & 266,285 & 280,284 & 292,301 & 288,345 & 297,346 & $336,347^{*}$ & 193,198 & 284,315 & $336,342^{*}$ & 266,279 & 280,284 & 292,301 \\
\hline MinAD & $6.86 \mathrm{E}-09$ & $6.45 \mathrm{E}-08$ & $5.31 \mathrm{E}-07$ & $1.37 \mathrm{E}-08$ & 4.33E-07 & $1.31 \mathrm{E}-06$ & $9.96 \mathrm{E}-09$ & $1.40 \mathrm{E}-07$ & $9.43 \mathrm{E}-07$ & $1.03 \mathrm{E}-08$ & $1.03 \mathrm{E}-07$ & $5.41 \mathrm{E}-07$ \\
\hline MinRD & 8.42E-08 & $7.91 \mathrm{E}-07$ & $6.51 \mathrm{E}-06$ & $1.55 \mathrm{E}-07$ & $4.03 \mathrm{E}-06$ & $1.18 \mathrm{E}-05$ & $6.74 \mathrm{E}-08$ & $1.05 \mathrm{E}-06$ & $7.31 \mathrm{E}-06$ & $1.83 \mathrm{E}-07$ & $1.50 \mathrm{E}-06$ & $7.10 \mathrm{E}-06$ \\
\hline Return & $\begin{array}{l}8.1523 \% \\
(2.53 \mathrm{E}-06)\end{array}$ & $\begin{array}{c}8.1546 \% \\
(1.08 \mathrm{E}-05)\end{array}$ & $\begin{array}{l}8.1466 \% \\
(6.00 \mathrm{E}-06)\end{array}$ & $\begin{array}{c}8.8667 \% \\
(4.13 \mathrm{E}-06)\end{array}$ & $\begin{array}{l}10.7388 \% \\
(1.46 \mathrm{E}-05)\end{array}$ & $\begin{array}{l}11.0988 \% \\
(4.04 \mathrm{E}-05)\end{array}$ & $\begin{array}{l}14.7832 \% \\
(3.18 \mathrm{E}-06)\end{array}$ & $\begin{array}{l}13.3495 \% \\
(1.37 \mathrm{E}-05)\end{array}$ & $\begin{array}{l}12.9037 \% \\
(5.95 \mathrm{E}-06)\end{array}$ & $\begin{array}{c}5.6248 \% \\
(3.23 \mathrm{E}-06)\end{array}$ & $\begin{array}{c}6.8994 \% \\
(1.30 \mathrm{E}-05)\end{array}$ & $\begin{array}{c}7.6217 \% \\
(1.01 \mathrm{E}-05)\end{array}$ \\
\hline $\mathrm{T} 1, \mathrm{~T} 2$ & $12,13^{*}$ & 69,117 & 177,212 & $12,13^{*}$ & 69,213 & 177,190 & $12,13^{*}$ & 67,302 & 211,244 & $12,13^{*}$ & 69,117 & 174,230 \\
\hline
\end{tabular}

Bullet portfolio

** Barbell portfolio

*** Both bullet and barbell portfolios 
active and passive portfolio management. The holding period returns of bullet and barbell portfolios are within one basis point of the target for both short and medium holding periods. Bullet portfolios have the closest returns to the target for short holding periods. Therefore, all portfolio formation strategies provide successful immunization for all holding periods. Nevertheless, as observed in Table 14, bullet and barbell portfolios produce closer returns to the target for short to medium holding periods.

These results on the performances of immunization portfolios under the humped volatility HJM framework suggest that any duration measure and any portfolio formation strategy is as successful as any other for any holding period. For all holding periods, with traditional as well as HJM duration measures, more than $97 \%$ of the portfolios are successfully immunized under the active portfolio management criterion. In this respect, the HJM duration measure does not provide any benefit over traditional duration measures.

As can be observed from Table 1, Panel D, when we use the humped volatility structure, similar to the exponential decay volatility, we find a negative volatility reduction factor. In other words, volatility is increasing exponentially with time to maturity. In this case, we also have another factor that increases volatility linearly with time to maturity. This factor, however, is less than $10^{-5}$, and therefore does not affect volatility significantly. The forward rate volatilities are $1.33 \%$ to $1.5 \%$ for one-year holding periods, $1.7 \%$ to $1.9 \%$ for five-year holding periods, and $1.75 \%$ to $2.33 \%$ for ten-year holding periods. These volatilities are not very high. This may be a reason for successful immunization with the duration matching strategy irrespective of the duration measure.

As shown in Table 13, bullet portfolios give the closest returns to the target for short holding periods with any risk measure. This is an expected result since, for short holding periods, term to maturity is the most important determinant of the duration measure. For medium to long holding periods, however, random portfolios are as successful as bullet and barbell portfolios in immunization. 


\section{Table 14}

\section{Performance of Duration Matched Bullet and Barbell Portfolios under Humped Volatility HJM Framework}

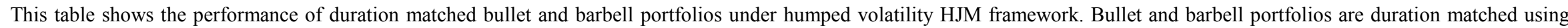

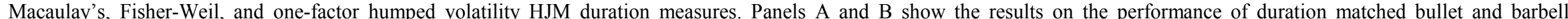

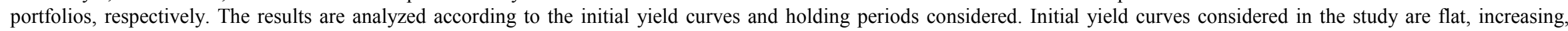

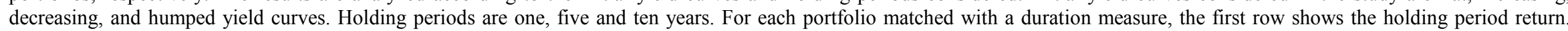
Standard errors of the returns are in parenthesis. The following two rows are the maximum absolute (MaxAD) and relative (MaxRD) deviations of portfolio returns from the target return.

\begin{tabular}{|c|c|c|c|c|c|c|c|c|c|c|c|c|}
\hline \multicolumn{13}{|c|}{ Panel A: Bullet Portfolios } \\
\hline & \multicolumn{3}{|c|}{$\begin{array}{c}\text { Flat } \\
\text { (August, 1989) }\end{array}$} & \multicolumn{3}{|c|}{$\begin{array}{c}\text { Increasing } \\
\text { (January, 1985) }\end{array}$} & \multicolumn{3}{|c|}{$\begin{array}{c}\text { Decreasing } \\
(\text { May, 1981) }\end{array}$} & \multicolumn{3}{|c|}{$\begin{array}{c}\text { Humped } \\
\text { (October, 1986) }\end{array}$} \\
\hline & 1 year & 5 year & 10 year & 1 year & 5 year & 10 year & 1 year & 5 year & 10 year & 1 year & 5 year & 10 year \\
\hline \multirow[t]{2}{*}{ Macaulay } & $8.1523 \%$ & $8.1548 \%$ & $8.1458 \%$ & $8.8667 \%$ & $10.7402 \%$ & $11.0343 \%$ & $14.7832 \%$ & $13.3499 \%$ & $12.8717 \%$ & $5.6248 \%$ & $6.8985 \%$ & $7.6253 \%$ \\
\hline & $(3.81 \mathrm{E}-06)$ & $(2.17 \mathrm{E}-04)$ & $(9.98 \mathrm{E}-05)$ & $(6.44 \mathrm{E}-06)$ & $(2.98 \mathrm{E}-04)$ & $(5.52 \mathrm{E}-04)$ & $(3.84 \mathrm{E}-06)$ & (1.10E-04) & $(4.56 \mathrm{E}-04)$ & $(5.31 \mathrm{E}-06)$ & $(4.12 \mathrm{E}-04)$ & $(1.18 \mathrm{E}-03)$ \\
\hline MaxAD & $1.61 \mathrm{E}-08$ & $2.46 \mathrm{E}-06$ & $7.73 \mathrm{E}-06$ & $3.20 \mathrm{E}-08$ & $1.40 \mathrm{E}-05$ & $6.46 \mathrm{E}-04$ & $1.54 \mathrm{E}-08$ & $4.39 \mathrm{E}-06$ & $3.19 \mathrm{E}-04$ & $2.73 \mathrm{E}-08$ & $9.07 \mathrm{E}-06$ & $3.55 \mathrm{E}-05$ \\
\hline MaxRD & $1.98 \mathrm{E}-07$ & $3.02 \mathrm{E}-05$ & $9.49 \mathrm{E}-05$ & $3.61 \mathrm{E}-07$ & $1.30 \mathrm{E}-04$ & $5.82 \mathrm{E}-03$ & $1.04 \mathrm{E}-07$ & $3.29 \mathrm{E}-05$ & $2.47 \mathrm{E}-03$ & $4.85 \mathrm{E}-07$ & $1.31 \mathrm{E}-04$ & $4.66 \mathrm{E}-04$ \\
\hline \multirow[t]{2}{*}{ Fisher-Weil } & $8.1523 \%$ & $8.1548 \%$ & $8.1460 \%$ & $8.8667 \%$ & $10.7401 \%$ & $10.0736 \%$ & $14.7832 \%$ & $13.3499 \%$ & $12.8905 \%$ & $5.6248 \%$ & $6.8981 \%$ & $7.6226 \%$ \\
\hline & $(3.80 \mathrm{E}-06)$ & $(2.20 \mathrm{E}-04)$ & $(1.00 \mathrm{E}-04)$ & $(6.43 \mathrm{E}-06)$ & $(2.52 \mathrm{E}-04)$ & $(1.06 \mathrm{E}-03)$ & $(3.84 \mathrm{E}-06)$ & $(1.11 \mathrm{E}-04)$ & $(9.63 \mathrm{E}-04)$ & $(5.31 \mathrm{E}-06)$ & $(5.65 \mathrm{E}-04)$ & $(1.60 \mathrm{E}-04)$ \\
\hline MaxAD & $1.61 \mathrm{E}-08$ & $2.41 \mathrm{E}-06$ & $5.73 \mathrm{E}-06$ & $3.19 \mathrm{E}-08$ & $1.27 \mathrm{E}-05$ & $1.03 \mathrm{E}-02$ & $1.54 \mathrm{E}-08$ & $4.74 \mathrm{E}-06$ & $1.31 \mathrm{E}-04$ & 2.73E-08 & $1.28 \mathrm{E}-05$ & $9.16 \mathrm{E}-06$ \\
\hline $\operatorname{MaxRD}$ & $1.97 \mathrm{E}-07$ & $2.96 \mathrm{E}-05$ & 7.04E-05 & $3.60 \mathrm{E}-07$ & $1.18 \mathrm{E}-04$ & $9.24 \mathrm{E}-02$ & $1.04 \mathrm{E}-07$ & $3.55 \mathrm{E}-05$ & $1.02 \mathrm{E}-03$ & 4.85E-07 & $1.86 \mathrm{E}-04$ & $1.20 \mathrm{E}-04$ \\
\hline \multirow[t]{2}{*}{ HJM } & $8.1523 \%$ & $8.1537 \%$ & $8.1508 \%$ & $8.8667 \%$ & $10.7394 \%$ & $11.2082 \%$ & $14.7832 \%$ & $13.3493 \%$ & $12.9631 \%$ & $5.6248 \%$ & $6.8985 \%$ & $7.6273 \%$ \\
\hline & $(2.53 \mathrm{E}-06)$ & $(4.47 \mathrm{E}-04)$ & $(1.03 \mathrm{E}-04)$ & $(4.13 \mathrm{E}-06)$ & (1.01E-04) & (1.09E-03) & $(3.18 \mathrm{E}-06)$ & (1.84E-04) & $(4.91 \mathrm{E}-04)$ & $(3.23 \mathrm{E}-06)$ & $(4.26 \mathrm{E}-04)$ & $(2.89 \mathrm{E}-05)$ \\
\hline MaxAD & $6.86 \mathrm{E}-09$ & $8.84 \mathrm{E}-06$ & $4.32 \mathrm{E}-05$ & $1.37 \mathrm{E}-08$ & $6.31 \mathrm{E}-06$ & $1.09 \mathrm{E}-03$ & $9.96 \mathrm{E}-09$ & $1.73 \mathrm{E}-06$ & $5.95 \mathrm{E}-04$ & $1.03 \mathrm{E}-08$ & $8.99 \mathrm{E}-06$ & $5.55 \mathrm{E}-05$ \\
\hline MaxRD & $8.42 \mathrm{E}-08$ & $1.08 \mathrm{E}-04$ & $5.30 \mathrm{E}-04$ & $1.55 \mathrm{E}-07$ & $5.87 \mathrm{E}-05$ & $9.84 \mathrm{E}-03$ & $6.74 \mathrm{E}-08$ & $1.29 \mathrm{E}-05$ & $4.61 \mathrm{E}-03$ & $1.83 \mathrm{E}-07$ & $1.30 \mathrm{E}-04$ & $7.28 \mathrm{E}-04$ \\
\hline
\end{tabular}


Table 14 - Continued

\begin{tabular}{|c|c|c|c|c|c|c|c|c|c|c|c|c|}
\hline \multicolumn{13}{|c|}{ Panel B: Barbell Portfolios } \\
\hline & \multicolumn{3}{|c|}{$\begin{array}{c}\text { Flat } \\
\text { (August, 1989) }\end{array}$} & \multicolumn{3}{|c|}{$\begin{array}{c}\text { Increasing } \\
\text { (January, 1985) }\end{array}$} & \multicolumn{3}{|c|}{$\begin{array}{c}\text { Decreasing } \\
\text { (May, 1981) }\end{array}$} & \multicolumn{3}{|c|}{$\begin{array}{c}\text { Humped } \\
\text { (October, 1986) }\end{array}$} \\
\hline & 1 year & 5 year & 10 year & 1 year & 5 year & 10 year & 1 year & 5 year & 10 year & 1 year & 5 year & 10 year \\
\hline \multirow[t]{2}{*}{ Macaulay } & $8.1523 \%$ & $8.1534 \%$ & $8.1414 \%$ & $8.8666 \%$ & $10.7364 \%$ & $11.0882 \%$ & $14.7832 \%$ & $13.3486 \%$ & $12.8999 \%$ & $5.6248 \%$ & $6.8971 \%$ & $7.6111 \%$ \\
\hline & $(8.66 \mathrm{E}-07)$ & $(1.80 \mathrm{E}-05)$ & $(7.33 \mathrm{E}-05)$ & $(1.31 \mathrm{E}-06)$ & $(2.91 \mathrm{E}-05)$ & (1.19E-04) & $(6.65 \mathrm{E}-07)$ & $(6.71 \mathrm{E}-06)$ & $(2.11 \mathrm{E}-05)$ & $(1.40 \mathrm{E}-06)$ & $(3.21 \mathrm{E}-05)$ & $(1.36 \mathrm{E}-04)$ \\
\hline MaxAD & $3.87 \mathrm{E}-07$ & $1.19 \mathrm{E}-05$ & $5.17 \mathrm{E}-05$ & 7.02E-07 & $2.36 \mathrm{E}-05$ & $1.08 \mathrm{E}-04$ & $2.58 \mathrm{E}-07$ & $8.93 \mathrm{E}-06$ & $3.71 \mathrm{E}-05$ & 7.49E-07 & $2.30 \mathrm{E}-05$ & $1.06 \mathrm{E}-04$ \\
\hline MaxRD & $4.75 \mathrm{E}-06$ & $1.46 \mathrm{E}-04$ & $6.34 \mathrm{E}-04$ & 7.92E-06 & $2.20 \mathrm{E}-04$ & $9.69 \mathrm{E}-04$ & $1.74 \mathrm{E}-06$ & $6.69 \mathrm{E}-05$ & $2.88 \mathrm{E}-04$ & $1.33 \mathrm{E}-05$ & $3.34 \mathrm{E}-04$ & $1.39 \mathrm{E}-03$ \\
\hline \multirow[t]{2}{*}{ Fisher-Weil } & $8.1523 \%$ & $8.1534 \%$ & $8.1414 \%$ & $8.8666 \%$ & $10.7364 \%$ & $11.0879 \%$ & $14.7832 \%$ & $13.3485 \%$ & $12.8994 \%$ & $5.6248 \%$ & $6.8972 \%$ & $7.6117 \%$ \\
\hline & (8.61E-07) & (1.77E-05) & $(7.17 \mathrm{E}-05)$ & $(1.31 \mathrm{E}-06)$ & $(2.95 \mathrm{E}-05)$ & $(1.25 \mathrm{E}-04)$ & $(6.80 \mathrm{E}-07)$ & $(8.40 \mathrm{E}-06)$ & $(3.24 \mathrm{E}-05)$ & $(1.34 \mathrm{E}-06)$ & $(3.01 \mathrm{E}-05)$ & $(1.26 \mathrm{E}-04)$ \\
\hline MaxAD & $3.85 \mathrm{E}-07$ & $1.19 \mathrm{E}-05$ & $5.10 \mathrm{E}-05$ & 7.02E-07 & $2.37 \mathrm{E}-05$ & $1.10 \mathrm{E}-04$ & $2.69 \mathrm{E}-07$ & $9.59 \mathrm{E}-06$ & $4.25 \mathrm{E}-05$ & 7.27E-07 & $2.22 \mathrm{E}-05$ & $1.00 \mathrm{E}-04$ \\
\hline MaxRD & 4.73E-06 & $1.45 \mathrm{E}-04$ & $6.26 \mathrm{E}-04$ & 7.91E-06 & $2.21 \mathrm{E}-04$ & $9.95 \mathrm{E}-04$ & $1.82 \mathrm{E}-06$ & $7.19 \mathrm{E}-05$ & $3.29 \mathrm{E}-04$ & $1.29 \mathrm{E}-05$ & $3.21 \mathrm{E}-04$ & $1.32 \mathrm{E}-03$ \\
\hline \multirow[t]{2}{*}{ HJM } & $8.1523 \%$ & $8.1540 \%$ & $8.1444 \%$ & $8.8666 \%$ & $10.7377 \%$ & $11.0945 \%$ & $14.7832 \%$ & $13.3488 \%$ & $12.9008 \%$ & $5.6248 \%$ & $6.8985 \%$ & $7.6182 \%$ \\
\hline & $(5.18 \mathrm{E}-07)$ & $(2.74 \mathrm{E}-06)$ & $(3.63 \mathrm{E}-06)$ & $(7.72 \mathrm{E}-07)$ & $(4.28 \mathrm{E}-06)$ & $(5.78 \mathrm{E}-06)$ & $(6.32 \mathrm{E}-07)$ & $(3.52 \mathrm{E}-06)$ & $(4.89 \mathrm{E}-06)$ & $(6.50 \mathrm{E}-07)$ & $(3.47 \mathrm{E}-06)$ & $(4.72 \mathrm{E}-06)$ \\
\hline MaxAD & $1.94 \mathrm{E}-07$ & $5.94 \mathrm{E}-06$ & $2.17 \mathrm{E}-05$ & $3.41 \mathrm{E}-07$ & $1.13 \mathrm{E}-05$ & $4.43 \mathrm{E}-05$ & $1.98 \mathrm{E}-07$ & $7.01 \mathrm{E}-06$ & $2.86 \mathrm{E}-05$ & $3.13 \mathrm{E}-07$ & $9.44 \mathrm{E}-06$ & $3.56 \mathrm{E}-05$ \\
\hline MaxRD & $2.38 \mathrm{E}-06$ & $7.28 \mathrm{E}-05$ & $2.67 \mathrm{E}-04$ & $3.84 \mathrm{E}-06$ & $1.05 \mathrm{E}-04$ & $3.99 \mathrm{E}-04$ & $1.34 \mathrm{E}-06$ & $5.25 \mathrm{E}-05$ & $2.21 \mathrm{E}-04$ & $5.56 \mathrm{E}-06$ & $1.37 \mathrm{E}-04$ & $4.67 \mathrm{E}-04$ \\
\hline
\end{tabular}




\subsection{Discussion on the Performances of Duration Matching Portfolios}

All of these results lead us to conclude that one cannot say that duration measures derived from HJM models always perform better than their traditional counterparts. The evidence shows that when forward rate volatilities are low, duration matching strategy is an accurate immunization strategy with traditional as well as HJM risk measures. When the volatilities are high, HJM duration measures perform better than traditional counterparts according to the active portfolio management criterion. According to passive portfolio management, however, the performances of HJM and traditional risk measures are similar to each other.

It is difficult to interpret the evidence related to the active portfolio management, since there may be two reasons that may lead to this evidence. It is possible that HJM measures are significantly better in determining interest rate risk than traditional duration measures. On the other hand, when there are large shifts in the yield curve, a duration matching strategy alone may not be sufficient for immunization. One should think of using a duration and convexity matching strategy for large shifts in the yield curve. To bring evidence to these alternative explanations, in the next section, we analyze the performances of duration and convexity matching strategies. If the underperformance of traditional risk measures under high volatility persists with a duration and convexity matching strategy, we can conclude that HJM risk measures have significant benefits over traditional risk measures. If, however, the performances of HJM and traditional risk measures are not significantly different when we use a duration and convexity matching strategy, then the under-performance of traditional measures can be attributed to the immunization strategy rather than the risk measure.

The results under passive portfolio management suggest that a duration matching strategy is not a very effective immunization strategy irrespective of the risk measure used. The percentage of duration-matched portfolios that have returns within one basis point of the target is never above $29 \%$ under all HJM frameworks except the humped volatility HJM framework. Under humped volatility HJM framework, more than $30 \%$ of the portfolios have returns within one basis point of the target. These results suggest that, assuming a linear relation between bond price and yield curve changes and using a first-order approximation is 
not enough to generate cash flows close to those of the target. Second-order approximations may be necessary and therefore as suggested by Christensen and Sorensen (1994), a duration and convexity matching strategy may lead immunization portfolios to have much alike cash flows to that of the target.

When we analyze the portfolio formation strategies, we observe that for short holding periods, regardless of the yield curve shape and the duration measure, in most cases, bullet portfolios have the closest returns to the target yield and are always immunized. This suggests that the immunization performance of bullet portfolios is related to the holding period rather than the volatility level. Therefore, if one constructs bullet portfolios for short holding periods, it is safe to say that any duration measure will be successful regardless of the HJM framework chosen for the evolution of forward rates. For medium to long holding periods, the differences in the performances of random, bullet and barbell portfolios are indistinguishable. 


\section{CHAPTER 8}

\section{PERFORMANCE OF DURATION AND CONVEXITY MATCHED PORTFOLIOS}

The scenarios considered in duration-matched portfolios are used for the duration and convexity matching strategy to observe the relative performances of these two immunization strategies. In this respect four one-factor HJM models, four initial yield curves and three holding periods lead to 48 scenarios. For each of these scenarios, one bullet, one barbell, and 100 random portfolios are formed.

The portfolios used for the duration matching strategy require two securities, whereas the portfolios used for the duration and convexity matching strategy require three. To use the portfolios considered in the duration matching strategy for the duration and convexity matching strategy as well, we select an additional security for each portfolio. For barbell portfolios, we add a medium term security. In this respect, we consider one-, ten- and twentyyear bonds for one year holding period, five-, twelve- and twenty-year bonds for five year holding period, and ten-, fifteen- and twenty-year bonds for ten year holding period, are considered. The bullet portfolios are formed from the bonds that have durations closest to that of the target bond. We consider four different HJM models, each having a different duration measure. For each initial yield curve considered, these duration measures lead to different bullet portfolios for each holding period. In each bullet portfolio, there are three securities. The bullet portfolios used for duration and convexity matching are given in Table 8 . We use the same random portfolios used for duration matching. For each random portfolio used in duration matching, a third security is chosen randomly for duration and convexity matching. This strategy is used to keep the portfolios used for different immunization strategies similar to each other. This will be useful while comparing the performances of different immunization strategies.

An immunization portfolio is considered successful if the return of the portfolio is greater than or equal to the yield of the target zero coupon bond minus five basis points. The 
duration measures are the ones used for the duration matching strategy. Their corresponding convexity measures are also used to match for both duration and convexity. The results of this immunization strategy are analyzed according to the underlying one-factor HJM model.

\subsection{Constant Volatility One-Factor HJM Framework}

Table 15 shows the results on the performance of the duration and convexity matched portfolios under the constant volatility HJM framework. Under the constant volatility HJM framework, the HJM duration and convexity reduce to the Fisher-Weil duration and its corresponding convexity. In this respect, for this specific volatility, there are two different duration and convexity measures considered: Macaulay's and Fisher-Weil's.

Table 15, Panel A presents the results on the performances of Macaulay's duration and convexity measures. According to the active portfolio management criterion considered in the study, a portfolio is successfully immunized if the holding period return of the portfolio is greater than the target yield minus five basis points. According to this criterion, the performance of the duration and convexity matching strategy with Macaulay's risk measure is not significantly different than the performance of the duration matching strategy for short to medium holding periods. Significant improvement is observed for long holding periods except for the case of a decreasing initial yield curve. As can be observed from Table 1, Panel A, the decreasing initial yield curve corresponds to the lowest volatility case. And therefore duration matching strategy provides successful immunization. When the duration and convexity matching strategy is used for ten-year holding periods, the number of successfully immunized portfolios stays the same for a flat initial yield curve and increases by $35 \%$ for an increasing initial yield curve. The increasing initial yield curve case has the highest volatility of 3.13\% among the initial yield curves considered. This shows that when the volatility is high, the convexity adjustment is important, especially for long holding periods. The convexity of a bond is positively related to its time to maturity. Therefore, as the holding period increases, the convexity adjustment becomes more important in immunization. Indeed this is what we observe when we use the duration and convexity matching strategy with Macaulay's duration. 


\section{Table 15}

\section{Performance of Duration and Convexity Matched Portfolios under Constant Volatility HJM Framework}

The performances of 102 duration and convexity matched portfolios are summarized. Two of the portfolios are bullet and barbell, and the rest are random portfolios. The benchmark portfolios are the ones that are matched with the Fisher-Weil duration and convexity. Under constant volatility, the HJM risk measures are the same as the Fisher-Weil risk measures. The other risk measures are the Macaulay's duration and convexity. The results are analyzed according to the initial yield curves and holding periods. Four initial yield curves are considered: Flat (August 1989); increasing (January 1985); decreasing (May 1981); and humped (October 1986). The three holding periods are one-, five-, and ten-years. Panel A presents the

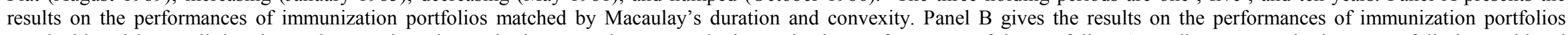
matched by Fisher-Weil duration and convexity. Three criteria are used to assess the immunization performances of the portfolios. According to one criterion, a portfolio is considered successful in immunization if the holding period return of the portfolio is greater than or equal to the yield of the target zero coupon bond minus 5 basis points. In Panels A and B, "\%

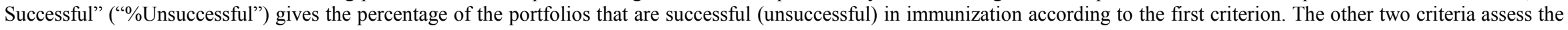
immunization performances by measuring the percentage of portfolios with returns within one and 100 basis points of the target yield. These are given in the next two rows. The maximum and minimum absolute (MaxAD and MinAD) and relative (MaxRD and MinRD) deviations from the target return are presented next. For each deviation, the returns of these portfolios, and the time to maturity of the bonds in the portfolios are given. Standard errors of the returns are in parentheses. Times to maturity are given in months, (T1, T2).

\begin{tabular}{|c|c|c|c|c|c|c|c|c|c|c|c|c|}
\hline \multicolumn{13}{|c|}{ Panel A: Macaulay's Duration and Convexity } \\
\hline & \multicolumn{3}{|c|}{$\begin{array}{c}\text { Flat } \\
\text { (August, 1989) } \\
\end{array}$} & \multicolumn{3}{|c|}{$\begin{array}{c}\text { Increasing } \\
\text { (January, 1985) }\end{array}$} & \multicolumn{3}{|c|}{$\begin{array}{l}\text { Decreasing } \\
\text { (May, 1981) }\end{array}$} & \multicolumn{3}{|c|}{$\begin{array}{c}\text { Humped } \\
\text { (October, 1986) }\end{array}$} \\
\hline & 1 year & 5 year & 10 year & 1 year & 5 year & 10 year & 1 year & 5 year & 10 year & 1 year & 5 year & 10 year \\
\hline$\%$ Successful & $98.04 \%$ & $97.06 \%$ & $95.10 \%$ & $97.06 \%$ & $96.08 \%$ & $85.29 \%$ & $96.08 \%$ & $91.18 \%$ & $82.35 \%$ & $96.08 \%$ & $95.10 \%$ & $74.51 \%$ \\
\hline Within 100 basis points & $100 \%$ & $99.02 \%$ & $100 \%$ & $99.02 \%$ & $98.04 \%$ & $99.02 \%$ & $98.04 \%$ & $94.12 \%$ & $98.04 \%$ & $90.20 \%$ & $91.18 \%$ & $93.14 \%$ \\
\hline Within 1 basis point & $57.84 \%{ }^{* * *}$ & $62.74 \%{ }^{*}$ & $55.88 \%^{* * *}$ & $45.10 \%{ }^{* * *}$ & $52.94 \%^{*}$ & $29.41 \%$ & $37.25 \%{ }^{* * *}$ & $48.04 \%^{*}$ & $32.35 \%$ & $36.27 \%^{* * *}$ & $45.10 \%^{*}$ & $14.71 \%{ }^{* *}$ \\
\hline MaxAD & $8.50 \mathrm{E}-03$ & $1.45 \mathrm{E}-02$ & $6.38 \mathrm{E}-03$ & $1.05 \mathrm{E}-02$ & $3.44 \mathrm{E}-02$ & $1.19 \mathrm{E}-02$ & 4.40E-02 & $4.06 \mathrm{E}-02$ & $2.47 \mathrm{E}-02$ & $2.28 \mathrm{E}-02$ & 4.89E-02 & 4.70E-02 \\
\hline MaxRD & $1.04 \mathrm{E}-01$ & $1.79 \mathrm{E}-01$ & 7.84E-02 & 1.19E-01 & $3.21 \mathrm{E}-01$ & $1.08 \mathrm{E}-01$ & 2.97E-01 & $3.04 \mathrm{E}-01$ & $1.91 \mathrm{E}-01$ & $4.06 \mathrm{E}-01$ & 7.08E-01 & $6.17 \mathrm{E}-01$ \\
\hline \multirow[t]{2}{*}{ Return } & $9.0024 \%$ & $6.6978 \%$ & $8.7850 \%$ & $7.8132 \%$ & $14.1829 \%$ & $12.2954 \%$ & $10.3863 \%$ & $9.2938 \%$ & $15.3697 \%$ & $7.9087 \%$ & $11.7871 \%$ & $2.9223 \%$ \\
\hline & $(1.25 \mathrm{E}-03)$ & $(6.59 \mathrm{E}-05)$ & (9.87E-04) & $(6.76 \mathrm{E}-03)$ & (8.48E-03) & $(1.08 \mathrm{E}-03)$ & $(3.54 \mathrm{E}-03)$ & $(8.10 \mathrm{E}-03)$ & $(2.43 \mathrm{E}-03)$ & $(2.71 \mathrm{E}-03)$ & $(4.84 \mathrm{E}-03)$ & (4.71E-03) \\
\hline $\mathrm{T} 1, \mathrm{~T} 2, \mathrm{~T} 3$ & $224,277,379$ & $182,236,277$ & $211,257,315$ & $130,203,333$ & $\begin{array}{c}60,144 \\
240^{* *}\end{array}$ & $177,190,210$ & $154,217,266$ & $176,210,217$ & $177,190,210$ & $138,266,314$ & $241,287,332$ & $\begin{array}{c}192,326 \\
342\end{array}$ \\
\hline MinAD & $2.34 \mathrm{E}-09$ & $1.14 \mathrm{E}-07$ & $1.52 \mathrm{E}-07$ & 7.92E-09 & 2.07E-09 & $1.75 \mathrm{E}-06$ & $8.52 \mathrm{E}-10$ & $3.95 \mathrm{E}-07$ & $1.30 \mathrm{E}-06$ & $6.94 \mathrm{E}-09$ & $1.67 \mathrm{E}-07$ & $1.04 \mathrm{E}-05$ \\
\hline MinRD & $2.88 \mathrm{E}-08$ & $1.40 \mathrm{E}-06$ & $1.87 \mathrm{E}-06$ & 8.93E-08 & $1.92 \mathrm{E}-08$ & $1.58 \mathrm{E}-05$ & $5.76 \mathrm{E}-09$ & $2.96 \mathrm{E}-06$ & $1.01 \mathrm{E}-05$ & $1.23 \mathrm{E}-07$ & $2.42 \mathrm{E}-06$ & $1.36 \mathrm{E}-04$ \\
\hline \multirow[t]{2}{*}{ Return } & $8.1523 \%$ & $8.1546 \%$ & $8.1465 \%$ & $8.8667 \%$ & $10.7388 \%$ & $11.0991 \%$ & $14.7832 \%$ & $13.3495 \%$ & $12.9035 \%$ & $5.6248 \%$ & $6.8994 \%$ & $7.6207 \%$ \\
\hline & $(1.25 \mathrm{E}-05)$ & $(7.45 \mathrm{E}-07)$ & $(1.43 \mathrm{E}-05)$ & $(2.04 \mathrm{E}-05)$ & (3.76E-06) & (8.45E-05) & $(1.20 \mathrm{E}-06)$ & $(6.60 \mathrm{E}-04)$ & $(3.64 \mathrm{E}-05)$ & $(1.81 \mathrm{E}-05)$ & $(1.14 \mathrm{E}-06)$ & (7.80E-05) \\
\hline $\mathrm{T} 1, \mathrm{~T} 2, \mathrm{~T} 3$ & $12,13,14^{*}$ & $67,216,277$ & $122,140,145$ & $12,13,14^{*}$ & $71,143,196$ & $254,261,329$ & $12,13,14^{*}$ & $83,84,86^{*}$ & $140,143,236$ & $12,13,14^{*}$ & $80,97,161$ & $\begin{array}{c}144,208 \\
211\end{array}$ \\
\hline
\end{tabular}

* Bullet portfolio; ** Barbell portfolio; *** Both bullet and barbell portfolios 
Table 15 - Continued

\begin{tabular}{|c|c|c|c|c|c|c|c|c|c|c|c|c|}
\hline \multicolumn{13}{|c|}{ Panel B: Fisher-Weil Duration and Convexity } \\
\hline & \multicolumn{3}{|c|}{$\begin{array}{c}\text { Flat } \\
\text { (August, 1989) }\end{array}$} & \multicolumn{3}{|c|}{$\begin{array}{c}\text { Increasing } \\
\text { (January, 1985) }\end{array}$} & \multicolumn{3}{|c|}{$\begin{array}{l}\text { Decreasing } \\
(\text { May, 1981) }\end{array}$} & \multicolumn{3}{|c|}{$\begin{array}{c}\text { Humped } \\
\text { (October, 1986) }\end{array}$} \\
\hline & 1 year & 5 year & 10 year & 1 year & 5 year & 10 year & 1 year & 5 year & 10 year & 1 year & 5 year & 10 year \\
\hline \%Successful & $98.04 \%$ & $99.02 \%$ & $100 \%$ & $97.06 \%$ & $97.06 \%$ & $98.04 \%$ & $100 \%$ & $99.02 \%$ & $97.06 \%$ & $98.04 \%$ & $98.04 \%$ & $100 \%$ \\
\hline Within 100 basis points & $99.02 \%$ & $100 \%$ & $100 \%$ & $100 \%$ & $100 \%$ & $99.02 \%$ & $100 \%$ & $100 \%$ & $99.02 \%$ & $100 \%$ & $100 \%$ & $100 \%$ \\
\hline Within 1 basis point & $77.45 \%^{* * *}$ & $96.08 \%{ }^{*}$ & $93.14 \%^{* * *}$ & $67.65 \%{ }^{* * *}$ & $90.20 \%^{*}$ & $89.22 \%^{* * *}$ & $84.31 \%^{* * *}$ & $94.12 \%{ }^{*}$ & $95.10 \%{ }^{* * *}$ & $59.80 \%^{* * *}$ & $93.14 \%{ }^{*}$ & $86.27 \%^{* * *}$ \\
\hline MaxAD & $1.15 \mathrm{E}-02$ & 4.43E-03 & $8.55 \mathrm{E}-04$ & $5.23 \mathrm{E}-03$ & $4.82 \mathrm{E}-03$ & $9.28 \mathrm{E}-03$ & $4.85 \mathrm{E}-04$ & $8.15 \mathrm{E}-03$ & $1.56 \mathrm{E}-03$ & $5.76 \mathrm{E}-03$ & $4.96 \mathrm{E}-03$ & $1.79 \mathrm{E}-03$ \\
\hline MaxRD & $1.41 \mathrm{E}-01$ & $5.43 \mathrm{E}-02$ & $1.05 \mathrm{E}-02$ & $5.90 \mathrm{E}-02$ & 4.49E-02 & 8.36E-02 & $3.28 \mathrm{E}-03$ & $6.10 \mathrm{E}-02$ & $6.19 \mathrm{E}-02$ & $1.02 \mathrm{E}-01$ & $7.20 \mathrm{E}-02$ & $2.35 \mathrm{E}-02$ \\
\hline \multirow[t]{2}{*}{ Return } & $7.005 \%$ & $7.7117 \%$ & $8.2320 \%$ & $9.3897 \%$ & $11.2211 \%$ & $12.0273 \%$ & $14.8318 \%$ & $12.5344 \%$ & $12.7476 \%$ & $6.2007 \%$ & $6.4029 \%$ & $7.8005 \%$ \\
\hline & (3.27E-03) & (5.72E-03) & (9.10E-04) & $(5.84 \mathrm{E}-03)$ & $(3.47 \mathrm{E}-06)$ & (9.33E-03) & $(5.82 \mathrm{E}-04)$ & (5.84E-03) & $(7.08 \mathrm{E}-06)$ & (8.45E-03) & (1.13E-03) & $(1.54 \mathrm{E}-03)$ \\
\hline $\mathrm{T} 1, \mathrm{~T} 2, \mathrm{~T} 3$ & $154,217,166$ & $180,312,319$ & $177,190,210$ & $50,316,323$ & $60,144,240^{* *}$ & $194,253,321$ & $110,223,294$ & $162,177,235$ & $174,230,245$ & $91,175,197$ & $129,134,227$ & $207,292,307$ \\
\hline $\operatorname{Min} A D$ & $2.37 \mathrm{E}-09$ & $1.97 \mathrm{E}-08$ & $1.68 \mathrm{E}-08$ & $4.27 \mathrm{E}-11$ & $1.52 \mathrm{E}-08$ & $6.44 \mathrm{E}-08$ & $9.63 \mathrm{E}-10$ & $2.34 \mathrm{E}-08$ & $3.78 \mathrm{E}-08$ & 3.19E-09 & $1.69 \mathrm{E}-07$ & $5.27 \mathrm{E}-08$ \\
\hline $\operatorname{MinRD}$ & $2.90 \mathrm{E}-08$ & $2.41 \mathrm{E}-07$ & 2.07E-07 & $4.82 \mathrm{E}-10$ & $1.42 \mathrm{E}-07$ & $5.81 \mathrm{E}-07$ & $6.51 \mathrm{E}-09$ & $1.75 \mathrm{E}-07$ & 2.93E-07 & $5.67 \mathrm{E}-08$ & $2.45 \mathrm{E}-06$ & $6.91 \mathrm{E}-07$ \\
\hline \multirow[t]{2}{*}{ Return } & $8.1523 \%$ & $8.1546 \%$ & $8.1465 \%$ & $8.8667 \%$ & $10.7388 \%$ & $11.0990 \%$ & $14.7832 \%$ & $13.3495 \%$ & $12.9036 \%$ & $5.6248 \%$ & $6.8994 \%$ & $7.6217 \%$ \\
\hline & $(1.25 \mathrm{E}-05)$ & (7.16E-07) & (3.01E-07) & $(2.57 \mathrm{E}-07)$ & $(5.00 \mathrm{E}-08)$ & (9.71E-06) & $(1.20 \mathrm{E}-05)$ & $(3.75 \mathrm{E}-07)$ & $(9.39 \mathrm{E}-06)$ & $(3.15 \mathrm{E}-06)$ & $(1.87 \mathrm{E}-06)$ & $(3.36 \mathrm{E}-06)$ \\
\hline $\mathrm{T} 1, \mathrm{~T} 2, \mathrm{~T} 3$ & $12,13,14^{*}$ & $110,173,197$ & $151,163,279$ & $15,22,184$ & $83,104,338$ & $126,200,312$ & $12,13,14^{*}$ & $66,214,250$ & $212,274,292$ & $22,27,190$ & $62,85,92$ & $123,142,345$ \\
\hline
\end{tabular}


In addition to the active portfolio management criterion, two passive portfolio management criteria are considered. The percentage of portfolios that have returns within 100 basis and one basis of the target yield are analyzed. As it was with the duration matching strategy, with duration and convexity matching strategy, more than $95 \%$ of the portfolios have returns within 100 basis points of the target. There is, however, a significant increase in the number of portfolios that have returns less than one basis point of the target yield when the duration and convexity matching strategy is used instead of the duration matching strategy. The number of portfolios that have returns within one basis point of the target yield increased by $30 \%$ to $50 \%$ for short to medium holding periods irrespective of the volatility. This increase is generally far less for long holding periods. This evidence is consistent with that of the Christensen and Sorensen (1994) study. Christensen and Sorensen show that when two portfolios have the same duration and convexity, the cash flows of the two portfolios are much alike. Our results show that the similarity between the cash flows is more apparent for short to medium holding periods. The minimum absolute and relative deviations shown in Table 15, Panel A also supports this evidence. The deviations with the duration and convexity matching strategy are hundred times lower than those with the duration matching strategy in most of the cases.

According to these results, a risk manager should prefer to match by both duration and convexity irrespective of the portfolio strategy. Under both active and passive portfolio management criteria, portfolios matched with both duration and convexity have better immunization performance. Also, Macaulay's duration and convexity perform well with the duration and convexity matching strategy. In this respect, adjusting for convexity when the volatility is high is important for active portfolio strategies. On the other hand, adjusting for convexity has the additional benefit for passive portfolio management irrespective of the level of the volatility, since duration and convexity matched portfolios have similar cash flows to that of the target.

As can be observed from Table 15 Panel A, and Table 16, when we match portfolios with Macaulay's duration and convexity measures, both bullet and barbell portfolios produce returns less than one basis point from the target yield for short holding periods. Bullet 
portfolios have a yield advantage whereas barbell portfolios have a convexity advantage. When we use the duration and convexity matching strategy, these advantages of bullet and barbell portfolios offset each other in the short term and both portfolios give holding period returns very close to the target yield.

Table 15, Panel B shows the performance of the strategy using Fisher-Weil duration and convexity matching. Under the active portfolio management criterion, the number of successfully immunized portfolios for a ten-year holding period with relatively high volatility $^{52}$ increases by a range of $30 \%$ to $40 \%$ with the duration and convexity matching strategy compared to the duration matching strategy. This increase is greater than that observed when using Macaulay's risk measures. Again, we observe that when there is high volatility, for non-infinitesimal changes in the yield curve, using the convexity term in addition to the duration measure improves the immunization performance of active portfolio strategies significantly. The better performance of the Fisher-Weil measures is expected, since they are the benchmark risk measures under the constant volatility HJM framework.

Under the passive portfolio management criterion, similar to the duration matching strategy, almost all portfolios that are duration and convexity matched with Fisher-Weil risk measures have returns within 100 basis points of the target yield. On the other hand, compared to the duration matching strategy, duration and convexity matching with Fisher-Weil risk measures increases the percentage of portfolios that have returns within one basis point of the target by a range of $60 \%$ to $80 \%$ for all holding periods irrespective of the volatility level. Again these results show that a portfolio matched with both duration and convexity has cash flows much like those of the target bond. Also, minimum absolute and relative deviations decreased significantly with duration and convexity matching using the Fisher-Weil measures. Minimum deviations reduced by 1,000 times for short holding periods, by a range of 50 to 100

\footnotetext{
${ }^{52}$ As can be observed from Table 1, Panel A, and as discussed in Chapter 6, Section 6.3, under the constant volatility HJM framework, high volatility is observed for increasing and humped initial yield curves. We consider volatility high if it is greater than $2.25 \%$ as discussed in Chapter 6, Section 6.3.
} 


\section{Table 16}

\section{Performance of Duration and Convexity Matched Bullet and Barbell Portfolios under Constant Volatility HJM Framework}

This table shows the performance of duration and convexity matched bullet and barbell portfolios under constant volatility HJM framework. Bullet and barbell portfolios are duration and convexity matched using Macaulay's and Fisher-Weil risk measures. HJM risk measures reduce to Fisher-Weil risk measures under constant volatility HJM framework. The results are analyzed according to the initial yield curves and holding periods considered. Initial yield curves considered in the study are flat, increasing, decreasing, and humped yield curves. Holding periods are one, five and ten years. For each portfolio matched with a duration measure, the first row shows the holding period return. Standard errors of the returns are in parenthesis. The following two rows are the maximum absolute (MaxAD) and relative (MaxRD) deviations of portfolio returns from the target return.

\begin{tabular}{|c|c|c|c|c|c|c|c|c|c|c|c|c|}
\hline & \multicolumn{3}{|c|}{$\begin{array}{c}\text { Flat } \\
\text { (August, 1989) }\end{array}$} & \multicolumn{3}{|c|}{$\begin{array}{c}\text { Increasing } \\
\text { (January, 1985) }\end{array}$} & \multicolumn{3}{|c|}{$\begin{array}{l}\text { Decreasing } \\
(\text { May, 1981) }\end{array}$} & \multicolumn{3}{|c|}{$\begin{array}{c}\text { Humped } \\
\text { (October, 1986) }\end{array}$} \\
\hline & 1 year & 5 year & 10 year & 1 year & 5 year & 10 year & 1 year & 5 year & 10 year & 1 year & 5 year & 10 year \\
\hline \multicolumn{13}{|l|}{ Bullet } \\
\hline \multirow[t]{2}{*}{ Macaulay } & $8.1523 \%$ & $8.1546 \%$ & $8.1435 \%$ & $8.8667 \%$ & $10.7388 \%$ & $10.9269 \%$ & $14.7832 \%$ & $13.3495 \%$ & $12.8423 \%$ & $5.6248 \%$ & $6.8996 \%$ & $7.5834 \%$ \\
\hline & $(1.25 \mathrm{E}-05)$ & (6.49E-04) & $(1.15 \mathrm{E}-04)$ & $(2.04 \mathrm{E}-05)$ & $(2.90 \mathrm{E}-04)$ & $(2.73 \mathrm{E}-04)$ & $(1.20 \mathrm{E}-05)$ & (6.60E-04) & (4.96E-04) & $(1.81 \mathrm{E}-05)$ & $(9.87 \mathrm{E}-04)$ & $(5.69 \mathrm{E}-04)$ \\
\hline MaxAD & $2.35 \mathrm{E}-09$ & $7.50 \mathrm{E}-07$ & $3.05 \mathrm{E}-05$ & 7.92E-09 & $2.10 \mathrm{E}-07$ & $1.72 \mathrm{E}-03$ & $8.52 \mathrm{E}-10$ & $3.95 \mathrm{E}-07$ & $6.13 \mathrm{E}-04$ & $6.94 \mathrm{E}-09$ & 2.09E-06 & $3.83 \mathrm{E}-04$ \\
\hline MaxRD & $2.88 \mathrm{E}-08$ & $9.20 \mathrm{E}-06$ & $3.75 \mathrm{E}-04$ & 8.93E-08 & $1.96 \mathrm{E}-06$ & $1.55 \mathrm{E}-02$ & 5.76E-09 & $2.96 \mathrm{E}-06$ & $4.75 \mathrm{E}-03$ & $1.23 \mathrm{E}-07$ & $3.03 \mathrm{E}-05$ & $5.02 \mathrm{E}-03$ \\
\hline \multirow[t]{2}{*}{ Fisher-Weil } & $8.1523 \%$ & $8.1545 \%$ & $8.1478 \%$ & $8.8667 \%$ & $10.7389 \%$ & $11.0994 \%$ & $14.7332 \%$ & $13.3493 \%$ & $12.9034 \%$ & $5.6248 \%$ & $6.8992 \%$ & $7.6226 \%$ \\
\hline & $(1.25 \mathrm{E}-05)$ & (6.47E-04) & (9.07E-05) & $(2.04 \mathrm{E}-05)$ & $(2.86 \mathrm{E}-04)$ & $(6.03 \mathrm{E}-07)$ & (1.20E-05) & $(6.75 \mathrm{E}-04)$ & (4.97E-07) & $(1.81 \mathrm{E}-05)$ & (9.84E-04) & (6.99E-04) \\
\hline MaxAD & 2.37E-09 & $1.0 \mathrm{E}-06$ & $1.32 \mathrm{E}-05$ & $7.80 \mathrm{E}-09$ & $1.16 \mathrm{E}-06$ & $4.61 \mathrm{E}-06$ & $9.63 \mathrm{E}-10$ & $1.85 \mathrm{E}-06$ & $2.02 \mathrm{E}-06$ & $6.96 \mathrm{E}-09$ & $2.04 \mathrm{E}-06$ & 8.54E-06 \\
\hline MaxRD & $2.90 \mathrm{E}-08$ & $1.23 \mathrm{E}-05$ & $1.62 \mathrm{E}-04$ & $8.80 \mathrm{E}-08$ & $1.08 \mathrm{E}-05$ & $4.16 \mathrm{E}-05$ & $6.51 \mathrm{E}-09$ & $1.39 \mathrm{E}-05$ & $1.57 \mathrm{E}-05$ & $1.24 \mathrm{E}-07$ & $2.95 \mathrm{E}-05$ & $1.12 \mathrm{E}-04$ \\
\hline \multicolumn{13}{|l|}{ Barbell } \\
\hline \multirow[t]{2}{*}{ Macaulay } & $8.1523 \%$ & $8.6591 \%$ & $8.1463 \%$ & $8.8667 \%$ & $14.1829 \%$ & $11.0872 \%$ & $14.7832 \%$ & $13.3498 \%$ & $12.8873 \%$ & $5.6248 \%$ & $6.8996 \%$ & $7.6155 \%$ \\
\hline & $(1.0 \mathrm{E}-12)$ & $(3.47 \mathrm{E}-04)$ & (4.93E-06) & $(9.80 \mathrm{E}-08)$ & $(8.48 \mathrm{E}-04)$ & $(9.56 \mathrm{E}-05)$ & (1.06E-07) & (3.02E-03) & (1.74E-04) & $(2.24 \mathrm{E}-08)$ & (8.44E-04) & $(7.01 \mathrm{E}-05)$ \\
\hline MaxAD & $4.17 \mathrm{E}-08$ & $5.04 \mathrm{E}-03$ & $2.03 \mathrm{E}-06$ & $1.33 \mathrm{E}-07$ & $3.44 \mathrm{E}-02$ & $1.17 \mathrm{E}-04$ & $2.18 \mathrm{E}-07$ & $3.09 \mathrm{E}-05$ & $1.63 \mathrm{E}-04$ & $1.57 \mathrm{E}-07$ & $3.11 \mathrm{E}-05$ & $6.21 \mathrm{E}-05$ \\
\hline MaxRD & $5.11 \mathrm{E}-07$ & $6.19 \mathrm{E}-02$ & $2.50 \mathrm{E}-05$ & $1.50 \mathrm{E}-06$ & $3.21 \mathrm{E}-01$ & $1.06 \mathrm{E}-03$ & $1.47 \mathrm{E}-06$ & $2.31 \mathrm{E}-04$ & $1.26 \mathrm{E}-03$ & 2.79E-06 & $4.51 \mathrm{E}-04$ & $8.15 \mathrm{E}-04$ \\
\hline \multirow[t]{2}{*}{ Fisher-Weil } & $8.1523 \%$ & $8.1888 \%$ & $8.1466 \%$ & $8.8667 \%$ & $11.2211 \%$ & $11.0987 \%$ & $14.7832 \%$ & $13.3893 \%$ & $12.9032 \%$ & $5.6248 \%$ & $6.7240 \%$ & $7.6218 \%$ \\
\hline & $(2.0 \mathrm{E}-08)$ & (3.01E-04) & (1.29E-07) & $(1.00 \mathrm{E}-12)$ & (1.86E-04) & $(1.38 \mathrm{E}-06)$ & $(6.00 \mathrm{E}-08)$ & $(2.75 \mathrm{E}-04)$ & $(3.46 \mathrm{E}-06)$ & $(9.30 \mathrm{E}-08)$ & $(2.73 \mathrm{E}-04)$ & (1.02E-07) \\
\hline MaxAD & $1.84 \mathrm{E}-08$ & $3.43 \mathrm{E}-04$ & $3.42 \mathrm{E}-07$ & $2.45 \mathrm{E}-08$ & $4.82 \mathrm{E}-03$ & $2.51 \mathrm{E}-06$ & 4.37E-08 & 3.99E-04 & $4.55 \mathrm{E}-06$ & $3.13 \mathrm{E}-08$ & $1.75 \mathrm{E}-03$ & $3.92 \mathrm{E}-07$ \\
\hline MaxRD & $2.26 \mathrm{E}-07$ & $4.20 \mathrm{E}-03$ & $4.20 \mathrm{E}-06$ & $2.76 \mathrm{E}-07$ & 4.49E-02 & $2.26 \mathrm{E}-05$ & $2.95 \mathrm{E}-07$ & $2.99 \mathrm{E}-03$ & $3.53 \mathrm{E}-05$ & $5.57 \mathrm{E}-07$ & $2.54 \mathrm{E}-02$ & $5.14 \mathrm{E}-06$ \\
\hline
\end{tabular}


times for medium holding periods, and by 1,000 times for long holding periods. This also suggests that duration and convexity matched portfolios have very similar cash flows to those of the target bond.

As can be observed from Table 15, Panel B, and Table 16, similar to Macaulay's risk measures, with Fisher-Weil risk measures, all bullet and barbell portfolios are successful for all holding periods under the active portfolio management criterion. Under the passive portfolio management criterion, bullet portfolios are also successfully immunized for all holding periods, whereas barbell portfolios are successfully immunized for short and long holding periods. Most of the random portfolios are also successful for all holding periods. Therefore, especially for medium to long holding periods, we cannot favor one portfolio formation strategy over another. For short holding periods, asset-liability managers can prefer bullet and barbell portfolios to alternative portfolio formation strategies.

Table 16 shows that bullet and barbell portfolios have similar performances for short and long holding periods with both Macaulay's and Fisher-Weil risk measures. For medium holding periods, bullet portfolios have returns closer to that of the target than do barbell portfolios. In this respect, bullet portfolios produce cash flows closer to the target than do barbell portfolios for medium holding periods. In the short and long run, however, both portfolio formation strategies have similar performance. Compared to the duration matching strategy, the maximum and relative deviations of bullet and barbell portfolios are reduced from 10 to 1000 times. This again shows the importance of matching with convexity in addition to duration.

The improvement in immunization performance for long holding periods with duration and convexity matching is more apparent with the Fisher-Weil measures. Since these are the benchmark measures that provide immunization against parallel shifts under all yield curve shapes, we would expect to observe better performance with the Fisher-Weil measures. The importance of the convexity adjustment for long holding periods is apparent with the FisherWeil measures as well. As expected, both bullet and barbell portfolios have very close returns to the target for short holding periods. Their yield and convexity advantages offset each other for short holding periods. It is interesting to observe that now bullet portfolios are not 
necessarily the ones with the closest returns to the target. According to Fuller and Settle (1984), term to maturity is the most important determinant of duration in the short run. This does not mean that it is the most important determinant of duration and convexity. This may be the reason for finding bullet portfolios as the ones with the closest returns to the target yield with the duration matching strategy but not with the duration and convexity matching strategy.

The results on the performance of the duration and convexity matching strategy under the constant volatility HJM framework suggest that the convexity adjustment is very important for long holding periods when there is high volatility for active portfolio strategies, and for all holding periods under the passive portfolio management criterion irrespective of the volatility. For medium to long holding periods, we cannot favor one portfolio formation strategy over another. For short holding periods, however, both bullet and barbell portfolios are always immunized and produce returns very close to the target, and therefore can be preferred to other alternatives.

\subsection{Exponential Volatility One-Factor HJM Framework}

The results on the performance of the duration and convexity matching strategy under the exponential decay volatility HJM framework are given in Table 17. Three different risk measures are considered. Two of these are the Macaulay's and Fisher-Weil measures. The last one is the benchmark exponential decay volatility HJM risk measures. The performances of Macaulay's, Fisher-Weil and HJM risk measures are given in Panels A, B and C, respectively.

The performances of Macaulay's duration and convexity measures are presented in Table 17, Panel A. According to the active portfolio management criterion, compared to the duration matching strategy, the main improvement is observed for long holding periods with high volatility. Under increasing and humped initial yield curves with ten year holding 


\section{Table 17}

\section{Performance of Duration and Convexity Matching Portfolios under Exponential Decay Volatility HJM Framework}

The performances of 102 duration and convexity matched portfolios are summarized. Two of the portfolios are bullet and barbell, and the rest are random portfolios. The benchmark portfolios are the ones that are matched according to the duration and convexity measures of the one factor HJM model with exponentially decaying volatility. The other two duration measures are the Macaulay's and the Fisher-Weil duration and convexity measures. Panel A, B, and C present the results the performances of immunization portfolios matched by Macaulay's duration and convexity, Fisher-Weil duration and convexity, and the exponential decay volatility one factor HJM duration and convexity, respectively. The results are analyzed according to the initial yield curves and holding periods. Four initial yield curves are considered: Flat (August 1989); increasing (January 1985); decreasing (May 1981); and humped (October 1986). The three holding periods are one-, five-, and ten-years. Three criteria are used to assess the immunization performances of the portfolios. According to one criterion, a portfolio is considered successful in immunization if the holding period return of the portfolio is greater than or equal to the yield of the target zero coupon bond minus 5 basis points. In Panels A and B, "\% Successful" ("\%Unsuccessful”) gives the percentage of the portfolios that are successful (unsuccessful) in immunization according to the first criterion. The other two criteria assess the immunization performances by measuring the percentage of portfolios with returns within one and 100 basis points of the target yield. These are given in the next two rows. The maximum and minimum absolute (MaxAD and MinAD) and relative (MaxRD and MinRD) deviations from the target return are presented next. For each deviation, the returns of these portfolios, and the time to maturity of the bonds in the portfolios are given. Standard errors of the returns are in parentheses. Times to maturity are given in months, (T1, T2).

\begin{tabular}{|c|c|c|c|c|c|c|c|c|c|c|c|c|}
\hline \multicolumn{13}{|c|}{ Panel A: Macaulay's Duration and Convexity } \\
\hline & \multicolumn{3}{|c|}{$\begin{array}{c}\text { Flat } \\
\text { (August, 1989) } \\
\end{array}$} & \multicolumn{3}{|c|}{$\begin{array}{c}\text { Increasing } \\
\text { (January, 1985) }\end{array}$} & \multicolumn{3}{|c|}{$\begin{array}{c}\text { Decreasing } \\
(\text { May, 1981) }\end{array}$} & \multicolumn{3}{|c|}{$\begin{array}{c}\text { Humped } \\
\text { (October, 1986) }\end{array}$} \\
\hline & 1 year & 5 year & 10 year & 1 year & 5 year & 10 year & 1 year & 5 year & 10 year & 1 year & 5 year & 10 year \\
\hline$\%$ Successful & $93.14 \%$ & $94.12 \%$ & $85.29 \%$ & $92.16 \%$ & $93.14 \%$ & $76.47 \%$ & $98.04 \%$ & $97.06 \%$ & $92.16 \%$ & $90.20 \%$ & $95.10 \%$ & $69.61 \%$ \\
\hline Within 100 basis points & $100.00 \%$ & $99.02 \%$ & $99.02 \%$ & $90.20 \%$ & $98.04 \%$ & $97.06 \%$ & $97.06 \%$ & $97.06 \%$ & $97.06 \%$ & $88.24 \%$ & $97.06 \%$ & $97.06 \%$ \\
\hline Within 1 basis point & $25.49 \% \%^{* * *}$ & $38.24 \%$ & $25.49 \%{ }^{* * *}$ & $18.63 \% \%^{* * *}$ & $35.29 \%$ & $10.78 \%^{* *}$ & $56.86 \% \%^{* * *}$ & $65.69 \%$ & $28.43 \%^{* *}$ & $14.71 \% \%^{* * *}$ & $32.35 \%$ & $8.82 \%^{* *}$ \\
\hline MaxAD & $8.12 \mathrm{E}-03$ & $3.24 \mathrm{E}-02$ & $2.31 \mathrm{E}-02$ & $6.48 \mathrm{E}-02$ & $1.98 \mathrm{E}-02$ & $1.60 \mathrm{E}-02$ & $6.05 \mathrm{E}-02$ & $4.16 \mathrm{E}-02$ & $3.98 \mathrm{E}-02$ & 4.34E-02 & $5.39 \mathrm{E}-02$ & $2.04 \mathrm{E}-02$ \\
\hline MaxRD & $9.96 \mathrm{E}-02$ & $3.97 \mathrm{E}-01$ & $2.84 \mathrm{E}-01$ & $7.31 \mathrm{E}-01$ & $1.85 \mathrm{E}-01$ & $1.44 \mathrm{E}-01$ & 4.09E-01 & $3.11 \mathrm{E}-01$ & $3.09 \mathrm{E}-01$ & 7.72E-01 & $7.81 \mathrm{E}-01$ & $2.68 \mathrm{E}-01$ \\
\hline \multirow[t]{2}{*}{ Return } & $8.9643 \%$ & $4.9158 \%$ & $10.4566 \%$ & $2.3831 \%$ & $12.7232 \%$ & $9.5040 \%$ & $8.7301 \%$ & $9.1925 \%$ & $8.9223 \%$ & $9.9672 \%$ & $12.2893 \%$ & $9.6620 \%$ \\
\hline & (4.61E-03) & $(3.24 \mathrm{E}-03)$ & $(2.93 \mathrm{E}-03)$ & $(5.23 \mathrm{E}-03)$ & (1.83E-03) & (1.64E-03) & $(1.13 \mathrm{E}-03)$ & $(4.10 \mathrm{E}-03)$ & $(2.59 \mathrm{E}-02)$ & $(4.84 \mathrm{E}-03)$ & $(5.38 \mathrm{E}-03)$ & $(2.19 \mathrm{E}-03)$ \\
\hline $\mathrm{T} 1, \mathrm{~T} 2, \mathrm{~T} 3$ & $185,244,307$ & $80,280,284$ & $170,181,229$ & $166,196,233$ & $241,287,332$ & $264,300,335$ & $154,217,266$ & $153,228,314$ & $140,277,289$ & $185,244,307$ & $157,248,317$ & $212,274,292$ \\
\hline $\operatorname{Min} A D$ & $9.19 \mathrm{E}-08$ & $9.08 \mathrm{E}-07$ & $3.37 \mathrm{E}-06$ & $1.78 \mathrm{E}-07$ & 8.87E-07 & $6.26 \mathrm{E}-06$ & $5.60 \mathrm{E}-08$ & $1.07 \mathrm{E}-06$ & 4.15E-06 & $1.73 \mathrm{E}-07$ & $1.36 \mathrm{E}-06$ & $9.32 \mathrm{E}-06$ \\
\hline MinRD & $1.13 \mathrm{E}-06$ & $1.11 \mathrm{E}-05$ & $4.13 \mathrm{E}-05$ & $2.01 \mathrm{E}-06$ & $8.26 \mathrm{E}-06$ & $5.64 \mathrm{E}-05$ & $3.79 \mathrm{E}-07$ & 7.98E-06 & $3.22 \mathrm{E}-05$ & $3.07 \mathrm{E}-06$ & $1.97 \mathrm{E}-05$ & $1.22 \mathrm{E}-04$ \\
\hline \multirow[t]{2}{*}{ Return } & $8.1523 \%$ & $8.1545 \%$ & $8.1462 \%$ & $8.8666 \%$ & $10.7389 \%$ & $11.0983 \%$ & $14.7832 \%$ & $13.3494 \%$ & $12.9040 \%$ & $5.6248 \%$ & $6.8995 \%$ & $7.6208 \%$ \\
\hline & (8.06E-06) & (6.91E-05) & $(5.06 \mathrm{E}-04)$ & (1.19E-05) & $(1.17 \mathrm{E}-04)$ & $(6.72 \mathrm{E}-04)$ & (9.19E-06) & $(3.31 \mathrm{E}-05)$ & (4.73E-04) & $(1.07 \mathrm{E}-05)$ & $(1.08 \mathrm{E}-04)$ & $(5.95 \mathrm{E}-04)$ \\
\hline $\mathrm{T} 1, \mathrm{~T} 2, \mathrm{~T} 3$ & $12,13,14^{*}$ & $69,73,213$ & $140,327,332$ & $12,13,14^{*}$ & $74,168,257$ & $224,245,270$ & $12,13,14^{*}$ & $69,73,213$ & $200,249,338$ & $12,13,14^{*}$ & $67,89,222$ & $126,143,248$ \\
\hline
\end{tabular}


Table 17 - Continued

\begin{tabular}{|c|c|c|c|c|c|c|c|c|c|c|c|c|}
\hline \multicolumn{13}{|c|}{ Panel B: Fisher-Weil Duration and Convexity } \\
\hline & \multicolumn{3}{|c|}{$\begin{array}{c}\text { Flat } \\
\text { (August, 1989) }\end{array}$} & \multicolumn{3}{|c|}{$\begin{array}{c}\text { Increasing } \\
\text { (January, 1985) }\end{array}$} & \multicolumn{3}{|c|}{$\begin{array}{l}\text { Decreasing } \\
\text { (May, 1981) }\end{array}$} & \multicolumn{3}{|c|}{$\begin{array}{c}\text { Humped } \\
\text { (October, 1986) }\end{array}$} \\
\hline & 1 year & 5 year & 10 year & 1 year & 5 year & 10 year & 1 year & 5 year & 10 year & 1 year & 5 year & 10 year \\
\hline$\%$ Successful & $92.16 \%$ & $95.10 \%$ & $88.24 \%$ & $91.18 \%$ & $95.10 \%$ & $77.45 \%$ & $97.06 \%$ & $98.04 \%$ & $87.25 \%$ & $90.20 \%$ & $93.14 \%$ & $74.51 \%$ \\
\hline Within 100 basis points & $98.04 \%$ & $99.02 \%$ & $99.02 \%$ & $95.10 \%$ & $97.06 \%$ & $98.04 \%$ & $99.02 \%$ & $100.00 \%$ & $99.02 \%$ & $93.14 \%$ & $98.04 \%$ & $96.08 \%$ \\
\hline Within 1 basis point & $24.51 \%{ }^{* * *}$ & $39.22 \%$ & $25.49 \%^{* * *}$ & $17.65 \%{ }^{* * *}$ & $34.31 \%$ & $15.69 \%{ }^{* *}$ & $44.12 \%^{* * *}$ & $60.78 \%$ & $46.08 \%{ }^{* * *}$ & $14.71 \%{ }^{* * *}$ & $32.35 \%$ & $10.78 \%{ }^{* *}$ \\
\hline MaxAD & 2.33E-02 & $1.05 \mathrm{E}-02$ & $1.45 \mathrm{E}-02$ & 4.09E-02 & $2.42 \mathrm{E}-02$ & $2.90 \mathrm{E}-02$ & $1.18 \mathrm{E}-02$ & $3.41 \mathrm{E}-03$ & 2.33E-02 & $4.26 \mathrm{E}-02$ & $4.66 \mathrm{E}-02$ & 4.67E-02 \\
\hline MaxRD & $2.86 \mathrm{E}-01$ & $1.28 \mathrm{E}-01$ & $1.78 \mathrm{E}-01$ & $4.62 \mathrm{E}-01$ & $2.26 \mathrm{E}-01$ & $2.61 \mathrm{E}-01$ & $8.01 \mathrm{E}-01$ & $2.56 \mathrm{E}-02$ & $1.80 \mathrm{E}-01$ & $7.58 \mathrm{E}-01$ & $6.76 \mathrm{E}-01$ & $6.13 \mathrm{E}-01$ \\
\hline \multirow[t]{2}{*}{ Return } & $10.4839 \%$ & $7.1074 \%$ & $9.5988 \%$ & $12.9591 \%$ & $8.3141 \%$ & $13.9979 \%$ & $2.9382 \%$ & $13.0083 \%$ & $15.2322 \%$ & $1.3638 \%$ & $11.5633 \%$ & $12.2946 \%$ \\
\hline & $(2.26 \mathrm{E}-03)$ & $(9.66 \mathrm{E}-03)$ & $(1.42 \mathrm{E}-02)$ & $(1.10 \mathrm{E}-02)$ & (7.47E-03) & (3.64E-04) & $(1.36 \mathrm{E}-02)$ & $(3.53 \mathrm{E}-03)$ & $(3.55 \mathrm{E}-03)$ & (7.35E-03) & (3.98E-02) & $(4.77 \mathrm{E}-02)$ \\
\hline $\mathrm{T} 1, \mathrm{~T} 2, \mathrm{~T} 3$ & $154,217,266$ & $196,304,331$ & $201,213,258$ & $154,187,265$ & $170,277,296$ & $127,292,324$ & $133,266,279$ & $182,210,276$ & $160,297,322$ & $185,244,307$ & $182,210,276$ & $264,300,335$ \\
\hline MinAD & $9.20 \mathrm{E}-08$ & $1.04 \mathrm{E}-06$ & $1.66 \mathrm{E}-06$ & $1.78 \mathrm{E}-07$ & $5.55 \mathrm{E}-10$ & $2.70 \mathrm{E}-05$ & $5.60 \mathrm{E}-08$ & $3.26 \mathrm{E}-07$ & 1.29E-06 & $1.73 \mathrm{E}-07$ & 4.99E-08 & 2.94E-07 \\
\hline MinRD & $1.13 \mathrm{E}-06$ & $1.28 \mathrm{E}-05$ & $2.03 \mathrm{E}-05$ & $2.01 \mathrm{E}-06$ & $5.17 \mathrm{E}-09$ & $2.43 \mathrm{E}-04$ & 3.79E-07 & $2.44 \mathrm{E}-06$ & $1.00 \mathrm{E}-05$ & $3.07 \mathrm{E}-06$ & $7.24 \mathrm{E}-07$ & $3.86 \mathrm{E}-06$ \\
\hline \multirow[t]{2}{*}{ Return } & $8.1523 \%$ & $8.1545 \%$ & $8.1464 \%$ & $8.8666 \%$ & $10.7388 \%$ & $11.0963 \%$ & $14.7832 \%$ & $13.3495 \%$ & $12.9038 \%$ & $5.6248 \%$ & $6.8994 \%$ & $7.6218 \%$ \\
\hline & $(8.05 \mathrm{E}-06)$ & (6.98E-05) & $(3.30 \mathrm{E}-04)$ & $(1.19 \mathrm{E}-05)$ & (1.07E-04) & $(5.34 \mathrm{E}-04)$ & $(9.19 \mathrm{E}-06)$ & $(3.41 \mathrm{E}-05)$ & $(1.72 \mathrm{E}-05)$ & (1.07E-05) & $(1.10 \mathrm{E}-04)$ & $(2.30 \mathrm{E}-04)$ \\
\hline $\mathrm{T} 1, \mathrm{~T} 2, \mathrm{~T} 3$ & $12,13,14^{*}$ & $69,73,213$ & $129,186,228$ & $12,13,14^{*}$ & $74,168,257$ & $140,250,267$ & $12,13,14^{*}$ & $68,88,332$ & $216,259,292$ & $12,13,14^{*}$ & $67,89,222$ & $120,180,240^{* *}$ \\
\hline
\end{tabular}

* Bullet portfolio

** Barbell portfolio

*** Both bullet and barbell portfolios 
Table 17 - Continued

\begin{tabular}{|c|c|c|c|c|c|c|c|c|c|c|c|c|}
\hline \multicolumn{13}{|c|}{ Panel C: HJM Exponential Decay Duration and Convexity } \\
\hline & \multicolumn{3}{|c|}{$\begin{array}{c}\text { Flat } \\
\text { (August, 1989) }\end{array}$} & \multicolumn{3}{|c|}{$\begin{array}{c}\text { Increasing } \\
\text { (January, 1985) }\end{array}$} & \multicolumn{3}{|c|}{$\begin{array}{c}\text { Decreasing } \\
(\text { May, 1981) }\end{array}$} & \multicolumn{3}{|c|}{$\begin{array}{c}\text { Humped } \\
\text { (October, 1986) }\end{array}$} \\
\hline & 1 year & 5 year & 10 year & 1 year & 5 year & 10 year & 1 year & 5 year & 10 year & 1 year & 5 year & 10 year \\
\hline \%Successful & $100.00 \%$ & $100.00 \%$ & $100.00 \%$ & $100.00 \%$ & $98.04 \%$ & $96.08 \%$ & $98.04 \%$ & $99.02 \%$ & $99.02 \%$ & $99.02 \%$ & $100.00 \%$ & $99.02 \%$ \\
\hline Within 100 basis points & $100.00 \%$ & $100.00 \%$ & $100.00 \%$ & $100.00 \%$ & $99.02 \%$ & $99.02 \%$ & $100.00 \%$ & $100.00 \%$ & $100.00 \%$ & $100.00 \%$ & $100.00 \%$ & $100.00 \%$ \\
\hline Within 1 basis point & $87.25 \%{ }^{* * *}$ & $99.02 \%$ & $95.10 \%{ }^{* * *}$ & $82.35 \%{ }^{* * *}$ & $92.16 \%$ & $87.25 \%{ }^{* * *}$ & $91.18 \%^{* * *}$ & $95.10 \%$ & $91.18 \%{ }^{* * *}$ & $84.31 \%{ }^{* * *}$ & $95.10 \%$ & $94.12 \%^{* * *}$ \\
\hline MaxAD & 5.99E-03 & 7.12E-04 & 2.32E-04 & $1.91 \mathrm{E}-03$ & $1.47 \mathrm{E}-03$ & $1.54 \mathrm{E}-03$ & 4.11E-03 & $1.27 \mathrm{E}-03$ & $6.20 \mathrm{E}-03$ & $1.29 \mathrm{E}-03$ & $2.83 \mathrm{E}-04$ & $5.61 \mathrm{E}-04$ \\
\hline MaxRD & 7.35E-02 & $8.73 \mathrm{E}-03$ & $2.85 \mathrm{E}-03$ & $2.16 \mathrm{E}-02$ & $1.37 \mathrm{E}-02$ & $1.39 \mathrm{E}-02$ & $2.78 \mathrm{E}-02$ & $9.54 \mathrm{E}-03$ & 4.81E-02 & $2.29 \mathrm{E}-02$ & 4.10E-03 & 7.36E-03 \\
\hline \multirow[t]{2}{*}{ Return } & $8.7518 \%$ & $8.2257 \%$ & $8.1698 \%$ & $9.0578 \%$ & $9.2650 \%$ & $9.5578 \%$ & $15.1941 \%$ & $13.2222 \%$ & $12.2832 \%$ & $5.4959 \%$ & $6.9277 \%$ & $7.5656 \%$ \\
\hline & $(1.15 \mathrm{E}-03)$ & (1.47E-03) & (3.14E-06) & (5.19E-03) & $(1.34 \mathrm{E}-03)$ & $(6.25 \mathrm{E}-04)$ & $(4.38 \mathrm{E}-03)$ & (1.43E-03) & $(6.36 \mathrm{E}-03)$ & $(2.40 \mathrm{E}-03)$ & $(4.68 \mathrm{E}-04)$ & $(9.10 \mathrm{E}-04)$ \\
\hline $\mathrm{T} 1, \mathrm{~T} 2, \mathrm{~T} 3$ & $140,266,288$ & $209,259,313$ & $224,245,270$ & $97,115,144$ & $180,312,319$ & $167,316,317$ & $154,217,266$ & $124,137,198$ & $207,292,307$ & $224,277,329$ & $124,137,198$ & $207,292,307$ \\
\hline $\operatorname{Min} A D$ & $7.23 \mathrm{E}-10$ & $1.99 \mathrm{E}-08$ & 4.16E-09 & $7.05 \mathrm{E}-10$ & 6.69E-09 & $1.96 \mathrm{E}-11$ & $4.95 \mathrm{E}-10$ & $7.46 \mathrm{E}-10$ & 4.71E-09 & $3.44 \mathrm{E}-10$ & $1.52 \mathrm{E}-08$ & $3.07 \mathrm{E}-09$ \\
\hline MinRD & 8.87E-09 & $2.44 \mathrm{E}-07$ & $5.11 \mathrm{E}-08$ & 7.96E-09 & $6.23 \mathrm{E}-08$ & $1.77 \mathrm{E}-10$ & 3.35E-09 & 5.59E-09 & $3.65 \mathrm{E}-08$ & $6.11 \mathrm{E}-09$ & $2.20 \mathrm{E}-07$ & $4.02 \mathrm{E}-08$ \\
\hline \multirow[t]{2}{*}{ Return } & $8.1523 \%$ & $8.1546 \%$ & $8.1465 \%$ & $8.8667 \%$ & $10.7388 \%$ & $11.0990 \%$ & $14.7832 \%$ & $13.3495 \%$ & $12.9036 \%$ & $5.6248 \%$ & $6.8994 \%$ & $7.6217 \%$ \\
\hline & $(6.89 \mathrm{E}-06)$ & (3.32E-08) & $(1.70 \mathrm{E}-07)$ & $(1.32 \mathrm{E}-07)$ & $(2.00 \mathrm{E}-08)$ & $(1.62 \mathrm{E}-06)$ & (8.71E-06) & (3.37E-07) & $(2.49 \mathrm{E}-05)$ & $(1.67 \mathrm{E}-06)$ & $(7.78 \mathrm{E}-07)$ & $(9.87 \mathrm{E}-07)$ \\
\hline $\mathrm{T} 1, \mathrm{~T} 2, \mathrm{~T} 3$ & $12,13,14^{*}$ & $74,224,286$ & $151,163,279$ & $15,22,184$ & $68,88,332$ & $126,186,244$ & $12,13,14^{*}$ & $66,214,250$ & $254,261,329$ & $22,27,190$ & $62,85,92$ & $134,185,251$ \\
\hline
\end{tabular}


periods, the exponential decay volatilities were above $2.25 \%$ and therefore considered as relatively high volatilities ${ }^{53}$. When volatilities are high, we cannot assume small shifts in the yield curve and therefore the convexity adjustment becomes necessary. Also, the convexity adjustment is more important for long holding periods because of the positive relation between convexity and time to maturity of the bond. In these respects, improvement in the immunization performance with duration and convexity matching for long holding periods should be expected. Compared to the duration matching strategy, with duration and convexity matching, the number of successful portfolios for the ten-year holding period increased by up to $60 \%$ at segments of the yield curves with high volatility.

Under the passive portfolio management criterion, the percentage of portfolios that have returns within 100 basis points is greater than $88 \%$. Therefore, when Macaulay's risk measures are used, the performance of duration and convexity matched portfolios is similar to that of duration matched portfolios. On the other hand, for short to medium holding periods, percentage of portfolios with returns within one basis point of the target is $8 \%$ to $45 \%$ higher with duration and convexity matching strategy compared to the duration matching strategy. For long holding periods, there is, $0 \%$ to $13 \%$ increase in the number of portfolios that have returns within one basis point of the target when the duration and convexity matching strategy is used instead of the duration matching strategy. In these respects, duration and convexity matching leads to cash flows that are much like to those of the target for short to medium holding periods when Macaulay's risk measures are used. For long holding periods, however, convexity adjustment is not enough to generate cash flows closer to that of the target with Macaulay's risk measures. Again this result shows that with duration and convexity matching we are able to imitate the cash flows of the target bond significantly better, especially for short to medium holding periods.

As can be observed from Table 17, Panel A, the minimum and maximum deviations of portfolio returns from the target are not very different between duration matching and duration-convexity matching strategies when Macaulay's risk measures are used. The minimum deviations of the duration and convexity matched portfolios at times are ten times

\footnotetext{
${ }^{53}$ See Chapter 6, Section 6.3. for a detailed explanation of volatility estimates and the categorization of volatilities as high and low.
} 
less than the minimum deviations of the duration matched portfolios. These results suggest that, although duration and convexity matching leads to similar cash flow structures to that of the target, the minimum and maximum deviations of portfolio returns are not much affected.

Also Table 17, Panel A shows that, similar to the duration matching strategy, bullet and barbell portfolios have returns within one basis point of the target yield for short holding periods. Additionally, barbell portfolios have returns within one basis point of the target yield for ten-year holding periods. All bullet portfolios are the ones with the closest returns to the target yield for short holding periods. This shows that in the short run, the most important determinant of duration is term to maturity, and term to maturity is also important for convexity. This contradicts with the result of the previous section and therefore we cannot say if term to maturity is also important for convexity or not.

The primary result related to Macaulay's risk measures is that, under the active portfolio management criterion, the performances of portfolios improve substantially with the duration and convexity matching strategy for long holding periods where forward rate volatilities are high. Under the passive portfolio management criterion, duration and convexity matching leads to better immunization performance for short to medium holding periods irrespective of the volatility level. In these respects, risk managers pursuing active or passive strategies should match with both duration and convexity. The duration and convexity matching strategy immunizes against non-infinitesimal changes in the yield curve especially when there is high volatility. Also, this strategy generates cash flows closer to those of the target especially for short to medium holding periods when Macaulay's risk measures are used. Additionally, risk managers can prefer bullet or barbell portfolios for short holding periods

Table 17, Panel B presents the results on the performance of Fisher-Weil duration and convexity. The results are similar to those on the performance of Macaulay's risk measures. According to the active portfolio management criterion, the main improvement with the duration and convexity matching strategy is observed for long holding periods when forward rate volatilities are high. Compared to the duration matching strategy, for ten-year holding 
periods with relatively high volatility ${ }^{54}$ the number of successful portfolios increased by a range of $44 \%$ to $68 \%$ with duration and convexity matching. Again, the importance of the convexity adjustment for high volatility and long holding periods is apparent.

Under the passive portfolio management criterion, the number of portfolios that have returns within one basis point of the target yield increases by a range of $7 \%$ to $35 \%$ irrespective of the holding period and volatility. More than $93 \%$ of the portfolios have returns within 100 basis points of the target. These results support the previous evidence that with the duration and convexity matching strategy, immunization portfolios have cash flows much similar to those of the target. Also minimum deviations reduce by a range of 10 to 10,000 times for medium to long holding periods. This also shows that with the duration and convexity matching strategy, portfolios have cash flows closer to those of the target bond.

When Fisher-Weil risk measures are used, both bullet and barbell portfolios have returns within one basis point of the target yield for one-year holding periods. All bullet and barbell portfolios and more than $74 \%$ of random portfolios are successful for all holding periods. The portfolios that have the closest returns to the target yield are again bullet portfolios for short holding periods. Nevertheless, barbell portfolios have returns that are within one basis of the target for long holding periods as well. In this respect, asset-liability managers may prefer bullet or barbell portfolios for short holding periods but for other holding periods, no one portfolio formation strategy is preferable to another.

These results are in line with the ones on Macaulay's duration and convexity. The duration and convexity matching strategy leads to a significant improvement in immunization performance. Under the active portfolio management criterion, the convexity adjustment is more important for long holding periods when volatility is high and therefore yield curve shifts are not infinitesimal. Under the passive portfolio management criterion, the convexity adjustment is important for all holding periods since with the duration and convexity matching strategy, portfolios generate cash flows that are closer to those of the target bond. Bullet and barbell portfolios have returns very close to that of the target for short holding periods.

\footnotetext{
${ }^{54}$ These correspond to ten year holding periods of increasing and humped initial yield curves, where forward rate volatilities are $2.34 \%$ and $2.28 \%$, respectively. Please refer to Table 1, Panel C, and Chapter 6, section 6.3 for details.
} 
Therefore, the yield advantage of bullet portfolios and the convexity advantage of barbell portfolios offset each other for short holding periods when the duration and convexity matching strategy is used.

The performance of the exponential decay volatility HJM duration and convexity is shown in Table 17, Panel C. Under the active portfolio management criterion, almost all portfolios are successful with the duration and convexity matching strategy. The most significant improvement is again observed for long holding periods where there is high forward rate volatility. The number of successfully immunized portfolios is at most $15 \%$ higher than it was with the duration matching strategy. This improvement is not as drastic as it is with traditional measures.

Under the passive portfolio management criterion, the improvement with HJM risk measures is drastic. Almost all portfolios have returns within 100 basis points of the target yield. Compared to the duration matching strategy, the number of portfolios that have returns less than one basis point of the target yield increases by a range of $70 \%$ to $85 \%$ with the duration and convexity matching strategy for all holding periods irrespective of the volatility. In this respect, when the correct risk measures are used, the main improvement is observed for passive strategies. With traditional risk measures, passive portfolios have improved performance as well, but with HJM measures, the improved performance is greater for all holding periods. In this respect, under the passive portfolio management criterion, the convexity adjustment is important but so is using the correct risk measure. The minimum deviations of the portfolio returns from the target yield also reduces by mostly more than 1000 times. This also supports the evidence that, with the duration and convexity matching strategy, immunization portfolios produce very close cash flows to those of the target for all holding periods.

Bullet and barbell portfolios have returns within one basis point of the target yield for short and long holding periods. This result supports the previous evidence that when the duration and convexity matching strategy is used, the yield advantage of bullet portfolios and the convexity advantage of barbell portfolios offset each other especially for short and long holding periods. 
As presented in Table 18, with the duration and convexity matching strategy, the performance of bullet and barbell portfolios improves for short and long holding periods when the HJM risk measures are used. The maximum deviations of the returns of bullet and barbell portfolios from the target yield reduces by 50 to 1,000 times when the HJM risk measures are used. There is not a significant reduction in the deviations when traditional risk measures are used. In these respects, when correct risk measures are used, the duration and convexity matching strategy reduces the deviation of portfolio returns for short and long holding periods. With traditional risk measures, however, this is not guaranteed.

These results lend further support to the conclusion that the convexity adjustment is important for long holding periods and for high volatility parts of the yield curve when active portfolio strategies are used. The results also confirm that the duration and convexity matching strategy generates cash flows much closer to those of the target. Therefore, the convexity adjustment is important for passive portfolio management for all holding periods irrespective of the volatility. These results hold with all risk measures considered. The differences between the traditional and the HJM risk measures are apparent under the passive portfolio management criterion. When we use the benchmark HJM risk measures with the duration and convexity matching strategy, we always obtain closer cash flows to those of the target bonds compared to the duration matching strategy. In other words, adding the secondorder term to the Taylor's series expansion of the bond price around the yield gives a better approximation. If, however, we add the second-order term using traditional measures, we may move farther away from the real value. Therefore, generating closer cash flows to the target with traditional duration and convexity measures is not guaranteed. This is also observed in the performances of bullet and barbell portfolios. Matching with the correct convexity measure reduces the deviation of portfolio returns from the target. This, however, might not be accomplished with traditional risk measures. Also, with the duration matching strategy, the bullet portfolios are the ones with the closest returns to the target yield mainly because time to maturity is the most important determinant of duration in the short term. It appears that time to maturity is generally important for both duration and convexity but there are cases in which this does not hold. Irrespective of the risk measures used in duration and convexity matching, 


\section{Table 18}

\section{Performance of Duration and Convexity Matched Bullet and Barbell Portfolios under Exponential Decay Volatility HJM Framework}

This table shows the performance of duration and convexity matched bullet and barbell portfolios under exponential decay volatility HJM framework. Bullet and barbell portfolios are duration and convexity matched using Macaulay's, Fisher-Weil, and one-factor exponential decay volatility HJM duration and convexity measures. Panels A and B show the results on the performance of duration and convexity matched bullet and barbell portfolios, respectively. The results are analyzed according to the initial yield curves and holding periods considered. Initial yield curves considered in the study are flat, increasing, decreasing, and humped yield curves. Holding periods are one, five and ten years. For each portfolio matched with a duration measure, the first row shows the holding period return. Standard errors of the returns are in parenthesis. The following two rows are the maximum absolute (MaxAD) and relative (MaxRD) deviations of the portfolio returns from the target return.

\begin{tabular}{|c|c|c|c|c|c|c|c|c|c|c|c|c|}
\hline \multicolumn{13}{|c|}{ Panel A: Bullet Portfolios } \\
\hline & \multicolumn{3}{|c|}{$\begin{array}{c}\text { Flat } \\
\text { (August, 1989) }\end{array}$} & \multicolumn{3}{|c|}{$\begin{array}{c}\text { Increasing } \\
(\text { January, 1985) }\end{array}$} & \multicolumn{3}{|c|}{$\begin{array}{c}\text { Decreasing } \\
(\text { May, 1981) }\end{array}$} & \multicolumn{3}{|c|}{$\begin{array}{c}\text { Humped } \\
\text { (October, 1986) } \\
\end{array}$} \\
\hline & 1 year & 5 year & 10 year & 1 year & 5 year & 10 year & 1 year & 5 year & 10 year & 1 year & 5 year & 10 year \\
\hline \multirow[t]{2}{*}{ Macaulay } & $8.1523 \%$ & $8.1945 \%$ & $8.1404 \%$ & $8.8666 \%$ & $10.7147 \%$ & $11.3391 \%$ & $14.7832 \%$ & $13.3358 \%$ & $12.8753 \%$ & $5.6248 \%$ & $6.9209 \%$ & $7.6011 \%$ \\
\hline & $(8.06 \mathrm{E}-06)$ & $(2.07 \mathrm{E}-03)$ & $(1.88 \mathrm{E}-03)$ & $(1.19 \mathrm{E}-05)$ & $(5.27 \mathrm{E}-04)$ & $(1.04 \mathrm{E}-03)$ & $(9.19 \mathrm{E}-06)$ & $(4.26 \mathrm{E}-04)$ & $(4.14 \mathrm{E}-04)$ & $(1.07 \mathrm{E}-05)$ & $(1.08 \mathrm{E}-03)$ & $(2.98 \mathrm{E}-04)$ \\
\hline MaxAD & $9.19 \mathrm{E}-08$ & 3.99E-04 & $6.16 \mathrm{E}-05$ & $1.78 \mathrm{E}-07$ & $2.41 \mathrm{E}-04$ & $2.40 \mathrm{E}-03$ & $5.60 \mathrm{E}-08$ & $1.37 \mathrm{E}-04$ & $2.83 \mathrm{E}-04$ & $1.73 \mathrm{E}-07$ & $2.15 \mathrm{E}-04$ & $2.06 \mathrm{E}-04$ \\
\hline $\operatorname{MaxRD}$ & $1.13 \mathrm{E}-06$ & 4.89E-03 & $7.57 \mathrm{E}-04$ & $2.01 \mathrm{E}-06$ & $2.24 \mathrm{E}-03$ & $2.16 \mathrm{E}-02$ & $3.79 \mathrm{E}-07$ & $1.03 \mathrm{E}-03$ & 2.19E-03 & $3.07 \mathrm{E}-06$ & $3.12 \mathrm{E}-03$ & $2.70 \mathrm{E}-03$ \\
\hline \multirow[t]{2}{*}{ Fisher-Weil } & $8.1523 \%$ & $8.1936 \%$ & $8.1379 \%$ & $8.8666 \%$ & $10.7153 \%$ & $11.2166 \%$ & $14.7832 \%$ & $13.3354 \%$ & $12.9050 \%$ & $5.6248 \%$ & $6.9206 \%$ & $7.6028 \%$ \\
\hline & $(8.05 \mathrm{E}-06)$ & $(2.06 \mathrm{E}-03)$ & $(1.81 \mathrm{E}-03)$ & $(1.18 \mathrm{E}-05)$ & $(5.18 \mathrm{E}-04)$ & $(3.86 \mathrm{E}-04)$ & $(9.19 \mathrm{E}-06)$ & $(4.30 \mathrm{E}-04)$ & $(5.75 \mathrm{E}-05)$ & $(1.07 \mathrm{E}-05)$ & (1.07E-03) & $(2.55 \mathrm{E}-04)$ \\
\hline MaxAD & $9.20 \mathrm{E}-08$ & $3.90 \mathrm{E}-04$ & 8.67E-05 & $1.78 \mathrm{E}-07$ & $2.35 \mathrm{E}-04$ & $1.18 \mathrm{E}-03$ & $5.61 \mathrm{E}-08$ & $1.41 \mathrm{E}-04$ & $1.37 \mathrm{E}-05$ & $1.73 \mathrm{E}-07$ & $2.12 \mathrm{E}-04$ & $1.89 \mathrm{E}-04$ \\
\hline MaxRD & $1.13 \mathrm{E}-06$ & $4.78 \mathrm{E}-03$ & $1.06 \mathrm{E}-03$ & $2.01 \mathrm{E}-06$ & $2.19 \mathrm{E}-03$ & $1.06 \mathrm{E}-02$ & $3.79 \mathrm{E}-07$ & $1.05 \mathrm{E}-03$ & $1.06 \mathrm{E}-04$ & $3.07 \mathrm{E}-06$ & $3.08 \mathrm{E}-03$ & $2.48 \mathrm{E}-03$ \\
\hline \multirow[t]{2}{*}{ HJM } & $8.1523 \%$ & $8.1845 \%$ & $8.1460 \%$ & $8.8667 \%$ & $10.7242 \%$ & $11.0993 \%$ & $14.7832 \%$ & $13.3383 \%$ & $12.9035 \%$ & $5.6248 \%$ & $6.9171 \%$ & $7.6221 \%$ \\
\hline & $(6.89 \mathrm{E}-06)$ & $(1.50 \mathrm{E}-03)$ & (9.37E-04) & $(1.04 \mathrm{E}-05)$ & $(4.54 \mathrm{E}-04)$ & $(6.38 \mathrm{E}-04)$ & $(8.71 \mathrm{E}-06)$ & (4.13E-04) & $(2.02 \mathrm{E}-07)$ & $(9.03 \mathrm{E}-06)$ & $(7.66 \mathrm{E}-04)$ & (3.83E-05) \\
\hline MaxAD & $7.23 \mathrm{E}-10$ & $2.99 \mathrm{E}-04$ & $4.95 \mathrm{E}-06$ & $2.08 \mathrm{E}-09$ & $1.46 \mathrm{E}-04$ & $3.30 \mathrm{E}-06$ & $4.95 \mathrm{E}-10$ & $1.12 \mathrm{E}-04$ & $1.46 \mathrm{E}-06$ & $1.76 \mathrm{E}-09$ & $1.77 \mathrm{E}-04$ & $3.90 \mathrm{E}-06$ \\
\hline MaxRD & 8.87E-09 & $3.67 \mathrm{E}-03$ & $6.07 \mathrm{E}-05$ & $2.35 \mathrm{E}-08$ & $1.36 \mathrm{E}-03$ & $2.97 \mathrm{E}-05$ & $3.35 \mathrm{E}-09$ & $8.39 \mathrm{E}-04$ & $1.13 \mathrm{E}-05$ & $3.14 \mathrm{E}-08$ & $2.56 \mathrm{E}-03$ & $5.12 \mathrm{E}-05$ \\
\hline
\end{tabular}


Table 18 - Continued

Panel B: Barbell Portfolios

\begin{tabular}{cccccccccccccc}
\hline \multicolumn{10}{c}{ Panel B: Barbell Portfolios } \\
\hline Macaulay & $8.1525 \%$ & $8.1945 \%$ & $8.1458 \%$ & $8.8670 \%$ & $10.7147 \%$ & $11.1071 \%$ & $14.7833 \%$ & $13.3358 \%$ & $12.8970 \%$ & $5.6252 \%$ & $6.9209 \%$ & $7.6269 \%$ \\
& $(1.01 \mathrm{E}-06)$ & $(2.07 \mathrm{E}-03)$ & $(1.49 \mathrm{E}-04)$ & $(1.54 \mathrm{E}-06)$ & $(5.27 \mathrm{E}-04)$ & $(2.80 \mathrm{E}-04)$ & $(6.55 \mathrm{E}-07)$ & $(4.26 \mathrm{E}-04)$ & $(2.11 \mathrm{E}-04)$ & $(1.47 \mathrm{E}-06)$ & $(1.08 \mathrm{E}-03)$ & $(2.79 \mathrm{E}-04)$ \\
MaxAD & $1.40 \mathrm{E}-06$ & $3.99 \mathrm{E}-04$ & $7.49 \mathrm{E}-06$ & $3.01 \mathrm{E}-06$ & $2.41 \mathrm{E}-04$ & $8.18 \mathrm{E}-05$ & $2.71 \mathrm{E}-07$ & $1.37 \mathrm{E}-04$ & $6.61 \mathrm{E}-05$ & $3.39 \mathrm{E}-06$ & $2.15 \mathrm{E}-04$ & $5.12 \mathrm{E}-05$ \\
MaxRD & $1.72 \mathrm{E}-05$ & $4.89 \mathrm{E}-03$ & $9.19 \mathrm{E}-05$ & $3.39 \mathrm{E}-05$ & $2.24 \mathrm{E}-03$ & $7.37 \mathrm{E}-04$ & $1.83 \mathrm{E}-06$ & $1.03 \mathrm{E}-03$ & $5.12 \mathrm{E}-04$ & $6.02 \mathrm{E}-05$ & $3.12 \mathrm{E}-03$ & $6.72 \mathrm{E}-04$ \\
Fisher-Weil & $8.1525 \%$ & $8.1936 \%$ & $8.1455 \%$ & $8.8670 \%$ & $10.7153 \%$ & $11.1049 \%$ & $14.7833 \%$ & $13.3354 \%$ & $12.9041 \%$ & $5.6252 \%$ & $6.9206 \%$ & $7.6218 \%$ \\
& $(1.03 \mathrm{E}-06)$ & $(2.06 \mathrm{E}-03)$ & $(1.45 \mathrm{E}-04)$ & $(1.46 \mathrm{E}-06)$ & $(5.18 \mathrm{E}-04)$ & $(2.17 \mathrm{E}-04)$ & $(4.99 \mathrm{E}-07)$ & $(4.30 \mathrm{E}-04)$ & $(6.53 \mathrm{E}-05)$ & $(1.55 \mathrm{E}-06)$ & $(1.07 \mathrm{E}-03)$ & $(2.30 \mathrm{E}-04)$ \\
MaxAD & $1.38 \mathrm{E}-06$ & $3.90 \mathrm{E}-04$ & $1.06 \mathrm{E}-05$ & $3.07 \mathrm{E}-06$ & $2.35 \mathrm{E}-04$ & $5.88 \mathrm{E}-05$ & $4.07 \mathrm{E}-07$ & $1.41 \mathrm{E}-04$ & $5.04 \mathrm{E}-06$ & $3.29 \mathrm{E}-06$ & $2.12 \mathrm{E}-04$ & $2.94 \mathrm{E}-07$ \\
MaxRD & $1.70 \mathrm{E}-05$ & $4.78 \mathrm{E}-03$ & $1.30 \mathrm{E}-04$ & $3.46 \mathrm{E}-05$ & $2.19 \mathrm{E}-03$ & $5.30 \mathrm{E}-04$ & $2.75 \mathrm{E}-06$ & $1.05 \mathrm{E}-03$ & $3.90 \mathrm{E}-05$ & $5.85 \mathrm{E}-05$ & $3.08 \mathrm{E}-03$ & $3.86 \mathrm{E}-06$ \\
HJM & $8.1523 \%$ & $8.1845 \%$ & $8.1465 \%$ & $8.8667 \%$ & $10.7242 \%$ & $11.0989 \%$ & $14.7832 \%$ & $13.3383 \%$ & $12.9034 \%$ & $5.6248 \%$ & $6.9171 \%$ & $7.6218 \%$ \\
& $(1.41 \mathrm{E}-08)$ & $(1.50 \mathrm{E}-03)$ & $(9.64 \mathrm{E}-08)$ & $(0.00 \mathrm{E}+00)$ & $(4.54 \mathrm{E}-04)$ & $(4.97 \mathrm{E}-07)$ & $(4.90 \mathrm{E}-08)$ & $(4.13 \mathrm{E}-04)$ & $(2.29 \mathrm{E}-06)$ & $(5.48 \mathrm{E}-08)$ & $(7.66 \mathrm{E}-04)$ & $(5.66 \mathrm{E}-08)$ \\
MaxAD & $5.47 \mathrm{E}-09$ & $2.99 \mathrm{E}-04$ & $1.58 \mathrm{E}-07$ & $5.40 \mathrm{E}-09$ & $1.46 \mathrm{E}-04$ & $5.48 \mathrm{E}-07$ & $2.17 \mathrm{E}-08$ & $1.12 \mathrm{E}-04$ & $2.34 \mathrm{E}-06$ & $7.57 \mathrm{E}-09$ & $1.77 \mathrm{E}-04$ & $2.72 \mathrm{E}-07$ \\
MaxRD & $6.71 \mathrm{E}-08$ & $3.67 \mathrm{E}-03$ & $1.94 \mathrm{E}-06$ & $6.09 \mathrm{E}-08$ & $1.36 \mathrm{E}-03$ & $4.93 \mathrm{E}-06$ & $1.47 \mathrm{E}-07$ & $8.39 \mathrm{E}-04$ & $1.82 \mathrm{E}-05$ & $1.35 \mathrm{E}-07$ & $2.56 \mathrm{E}-03$ & $3.57 \mathrm{E}-06$ \\
\hline
\end{tabular}


bullet and barbell portfolios produce returns within one basis point of the target yield for short holding periods. With the duration matching strategy, bullet portfolios are the ones that have closer returns to the target yield. This shows that when adjusted for convexity, the yield advantage of bullet and the convexity advantage of barbell portfolios offset each other and both produce returns very close to the target yield for short holding periods. For medium to long holding periods, we cannot favor one portfolio formation strategy over another.

\subsection{Constant Decay Volatility One-Factor HJM Framework}

Table 19, Panels A, B and C show the results on the performances of duration and convexity matching strategies with the Macaulay, Fisher-Weil, and constant decay volatility HJM risk measures, respectively. Since forward rates are simulated according to the constant decay volatility one-factor HJM model, the corresponding HJM risk measures provide the benchmark.

Under the constant decay volatility HJM framework, forward rate volatilities decline with time to maturity. Therefore, the highest volatilities are observed for short holding periods, and the lowest volatilities are observed for long holding periods. In these respects, we expect to observe some improvement by using the duration and convexity matching strategy for short holding periods. We also expect to observe an increase in the number of portfolios that produce closer returns to the target.

The performance of Macaulay's duration and convexity under the constant decay volatility HJM framework is presented in Table 19, Panel A. Under the active portfolio management criterion, the most significant improvement is observed for short holding periods when forward rate volatilities are high. This is followed by the improvement in medium-term holding periods. For one-year holding periods, the number of successfully immunized portfolios increases on the average by $40 \%$ compared to the duration matching strategy. There is a slight deterioration of immunization performance for long holding periods when forward rate volatilities are low. 


\section{Table 19}

\section{Performance of Duration and Convexity Matched Portfolios under Constant Decay Volatility HJM Framework}

The performances of 102 duration and convexity matched portfolios are summarized. Two of the portfolios are bullet and barbell, and the rest are random portfolios. The benchmark portfolios are the ones that are matched according to the duration and convexity measures of the one factor HJM model with constant decay volatility. The other two duration measures are the Macaulay's and the Fisher-Weil duration and convexity measures. Panel A, B, and C present the results the performances of immunization portfolios matched by Macaulay's duration and convexity, Fisher-Weil duration and convexity, and the constant decay volatility one factor HJM duration and convexity, respectively. The results are analyzed according to the initial yield curves and holding periods. Four initial yield curves are considered: Flat (August 1989); increasing (January 1985); decreasing (May 1981); and humped (October 1986). The three holding periods are one-, five-, and ten-years. Three criteria are used to assess the immunization performances of the portfolios. According to one criterion, a portfolio is considered successful in immunization if the holding period return of the portfolio is greater than or equal to the yield of the target zero coupon bond minus 5 basis points. In Panels A and B, "\% Successful” ("\%Unsuccessful”) gives the percentage of the portfolios that are successful (unsuccessful) in immunization according to the first criterion. The other two criteria assess the immunization performances by measuring the percentage of portfolios with returns within one and 100 basis points of the target yield. These are given in the next two rows. The maximum and minimum absolute (MaxAD and MinAD) and relative (MaxRD and MinRD) deviations from the target return are presented next. For each deviation, the returns of these portfolios, and the time to maturity of the bonds in the portfolios are given. Standard errors of the returns are in parentheses. Times to maturity are given in months, (T1, T2).

\begin{tabular}{|c|c|c|c|c|c|c|c|c|c|c|c|c|}
\hline \multicolumn{13}{|c|}{ Panel A: Macaulay’s Duration and Convexity } \\
\hline & \multicolumn{3}{|c|}{$\begin{array}{c}\text { Flat } \\
\text { (August, 1989) }\end{array}$} & \multicolumn{3}{|c|}{$\begin{array}{c}\text { Increasing } \\
\text { (January, 1985) }\end{array}$} & \multicolumn{3}{|c|}{$\begin{array}{c}\text { Decreasing } \\
\text { (May, 1981) }\end{array}$} & \multicolumn{3}{|c|}{$\begin{array}{c}\text { Humped } \\
\text { (October, 1986) }\end{array}$} \\
\hline & 1 year & 5 year & 10 year & 1 year & 5 year & 10 year & 1 year & 5 year & 10 year & 1 year & 5 year & 10 year \\
\hline$\%$ Successful & $98.04 \%$ & $92.16 \%$ & $83.33 \%$ & $77.45 \%$ & $82.35 \%$ & $85.29 \%$ & $87.25 \%$ & $86.27 \%$ & $81.37 \%$ & $79.41 \%$ & $80.39 \%$ & $81.37 \%$ \\
\hline Within 100 basis points & $100.00 \%$ & $94.12 \%$ & $86.27 \%$ & $95.10 \%$ & $88.24 \%$ & $80.39 \%$ & $96.08 \%$ & $86.27 \%$ & $90.20 \%$ & $90.20 \%$ & $85.29 \%$ & $83.33 \%$ \\
\hline Within 1 basis point & $0.00 \%$ & $2.94 \%$ & $0.98 \%$ & $9.80 \%$ & $0.00 \%$ & $0.00 \%$ & $22.55 \%{ }^{* * *}$ & $0.98 \%$ & $0.00 \%$ & $7.84 \%$ & $0.00 \%$ & $0.00 \%$ \\
\hline $\operatorname{MaxAD}$ & $3.85 \mathrm{E}-03$ & 4.11E-02 & $3.16 \mathrm{E}-02$ & 7.19E-02 & 4.40E-02 & $8.21 \mathrm{E}-02$ & $1.05 \mathrm{E}-02$ & $1.32 \mathrm{E}-02$ & $4.51 \mathrm{E}-02$ & 4.90E-02 & $6.33 \mathrm{E}-02$ & $4.41 \mathrm{E}-02$ \\
\hline MaxRD & 4.73E-02 & $5.03 \mathrm{E}-01$ & $3.88 \mathrm{E}-01$ & $8.11 \mathrm{E}-01$ & 4.10E-01 & 7.39E-01 & $7.12 \mathrm{E}-01$ & $9.90 \mathrm{E}-01$ & $3.49 \mathrm{E}-01$ & $8.71 \mathrm{E}-01$ & $9.17 \mathrm{E}-01$ & $5.78 \mathrm{E}-01$ \\
\hline \multirow[t]{2}{*}{ Return } & $7.7670 \%$ & $4.0492 \%$ & $4.9864 \%$ & $1.6772 \%$ & $15.1408 \%$ & $2.8922 \%$ & $15.8308 \%$ & $14.6657 \%$ & $8.3968 \%$ & $10.5238 \%$ & $0.5724 \%$ & $3.2130 \%$ \\
\hline & (5.41E-03) & $(4.27 \mathrm{E}-03)$ & (7.06E-03) & $(1.00 \mathrm{E}-02)$ & $(4.54 \mathrm{E}-03)$ & $(8.42 \mathrm{E}-02)$ & $(2.10 \mathrm{E}-02)$ & $(1.45 \mathrm{E}-02)$ & $(4.55 \mathrm{E}-02)$ & $(4.07 \mathrm{E}-02)$ & (3.49E-02) & (3.08E-02) \\
\hline $\mathrm{T} 1, \mathrm{~T} 2, \mathrm{~T} 3$ & $96,147,247$ & $137,147,230$ & $227,243,292$ & $128,184,252$ & $170,277,296$ & $194,253,321$ & $91,175,197$ & $132,176,217$ & $140,277,289$ & $125,231,312$ & $186,234,337$ & $127,130,241$ \\
\hline $\operatorname{Min} A D$ & 7.33E-04 & $5.66 \mathrm{E}-05$ & $3.62 \mathrm{E}-06$ & $1.76 \mathrm{E}-05$ & $1.30 \mathrm{E}-04$ & $4.26 \mathrm{E}-04$ & $1.56 \mathrm{E}-05$ & $4.16 \mathrm{E}-05$ & $1.80 \mathrm{E}-04$ & $3.96 \mathrm{E}-05$ & $3.10 \mathrm{E}-04$ & $2.10 \mathrm{E}-04$ \\
\hline MinRD & 8.99E-03 & $6.94 \mathrm{E}-04$ & $4.45 \mathrm{E}-05$ & 1.99E-04 & $1.21 \mathrm{E}-03$ & $3.84 \mathrm{E}-03$ & $1.05 \mathrm{E}-04$ & $3.12 \mathrm{E}-04$ & $1.40 \mathrm{E}-03$ & 7.03E-04 & $4.49 \mathrm{E}-03$ & $2.76 \mathrm{E}-03$ \\
\hline \multirow[t]{2}{*}{ Return } & $8.0790 \%$ & $8.1602 \%$ & $8.1462 \%$ & $8.8684 \%$ & $10.7258 \%$ & $11.1416 \%$ & $14.7817 \%$ & $13.3453 \%$ & $12.8856 \%$ & $5.6288 \%$ & $6.9304 \%$ & $7.6007 \%$ \\
\hline & (1.02E-03) & $(1.21 \mathrm{E}-03)$ & (1.22E-03) & (1.09E-03) & (3.05E-03) & $(1.31 \mathrm{E}-04)$ & (8.02E-03) & $(9.36 \mathrm{E}-03)$ & $(2.79 \mathrm{E}-04)$ & $(8.93 \mathrm{E}-03)$ & $(1.49 \mathrm{E}-03)$ & $(1.71 \mathrm{E}-03)$ \\
\hline $\mathrm{T} 1, \mathrm{~T} 2, \mathrm{~T} 3$ & $100,222,339$ & $119,307,309$ & $177,190,210$ & $59,259,319$ & $81,89,277$ & $130,320,346$ & $93,124,244$ & $162,177,235$ & $\begin{array}{c}324,330 \\
336^{*} \\
\end{array}$ & $49,136,266$ & $119,307,309$ & $201,213,258$ \\
\hline
\end{tabular}

* Bullet portfolio

** Barbell portfolio

*** Both bullet and barbell portfolios 
Table 19 - Continued

\begin{tabular}{|c|c|c|c|c|c|c|c|c|c|c|c|c|}
\hline \multicolumn{13}{|c|}{ Panel B: Fisher-Weil Duration and Convexity } \\
\hline & \multicolumn{3}{|c|}{$\begin{array}{c}\text { Flat } \\
\text { (August, 1989) } \\
\end{array}$} & \multicolumn{3}{|c|}{$\begin{array}{c}\text { Increasing } \\
\text { (January, 1985) }\end{array}$} & \multicolumn{3}{|c|}{$\begin{array}{c}\text { Decreasing } \\
\text { (May, 1981) }\end{array}$} & \multicolumn{3}{|c|}{$\begin{array}{c}\text { Humped } \\
\text { (October, 1986) }\end{array}$} \\
\hline & 1 year & 5 year & 10 year & 1 year & 5 year & 10 year & 1 year & 5 year & 10 year & 1 year & 5 year & 10 year \\
\hline$\%$ Successful & $98.04 \%$ & $90.20 \%$ & $87.25 \%$ & $63.73 \%$ & $81.37 \%$ & $90.20 \%$ & $74.51 \%$ & $85.29 \%$ & $76.47 \%$ & $63.73 \%$ & $82.35 \%$ & $92.16 \%$ \\
\hline Within 100 basis points & $100.00 \%$ & $97.06 \%$ & $83.33 \%$ & $96.08 \%$ & $91.18 \%$ & $88.24 \%$ & $96.08 \%$ & $89.22 \%$ & $84.31 \%$ & $97.06 \%$ & $93.14 \%$ & $84.31 \%$ \\
\hline Within 1 basis point & $0.00 \%$ & $5.88 \%$ & $0.00 \%$ & $8.82 \%$ & $2.94 \%$ & $0.00 \%$ & $40.20 \%{ }^{* * *}$ & $4.90 \%$ & $2.94 \%$ & $8.82 \%$ & $3.92 \%$ & $0.00 \%$ \\
\hline MaxAD & $8.45 \mathrm{E}-03$ & $4.52 \mathrm{E}-02$ & $6.21 \mathrm{E}-02$ & $2.60 \mathrm{E}-02$ & $7.20 \mathrm{E}-02$ & $1.08 \mathrm{E}-02$ & $1.41 \mathrm{E}-02$ & 5.89E-02 & $5.67 \mathrm{E}-02$ & $2.43 \mathrm{E}-02$ & $5.99 \mathrm{E}-02$ & $4.38 \mathrm{E}-02$ \\
\hline MaxRD & $1.04 \mathrm{E}-02$ & $5.54 \mathrm{E}-01$ & $7.62 \mathrm{E}-01$ & $2.93 \mathrm{E}-01$ & $6.70 \mathrm{E}-01$ & $9.70 \mathrm{E}-02$ & $9.55 \mathrm{E}-02$ & 4.41E-01 & 4.39E-01 & $4.33 \mathrm{E}-01$ & $8.68 \mathrm{E}-01$ & $5.75 \mathrm{E}-01$ \\
\hline \multirow[t]{2}{*}{ Return } & $7.3068 \%$ & $12.6718 \%$ & $1.9372 \%$ & $6.2703 \%$ & $3.5424 \%$ & $12.1709 \%$ & $16.1968 \%$ & $7.4629 \%$ & $18.5728 \%$ & $3.1911 \%$ & $0.9136 \%$ & $12.0004 \%$ \\
\hline & $(1.26 \mathrm{E}-03)$ & $(2.54 \mathrm{E}-03)$ & (8.40E-03) & (8.98E-03) & (8.20E-03) & & & (3.57E-03 & (5.72E-03 & $(6.54 \mathrm{E}-02)$ & $(3.00 \mathrm{E}-02)$ & $(2.91 \mathrm{E}-02)$ \\
\hline $\mathrm{T} 1, \mathrm{~T} 2, \mathrm{~T} 3$ & $\begin{array}{c}100,222 \\
339\end{array}$ & $\begin{array}{c}196,304 \\
331\end{array}$ & $\begin{array}{c}159,209 \\
228\end{array}$ & $\begin{array}{c}133,266 \\
279\end{array}$ & $\begin{array}{c}182,236 \\
277\end{array}$ & $\begin{array}{c}174,230 \\
245\end{array}$ & $\begin{array}{c}133,266 \\
279\end{array}$ & $\begin{array}{c}161,224 \\
335\end{array}$ & $\begin{array}{c}211,257 \\
315\end{array}$ & $\begin{array}{c}148,238 \\
266\end{array}$ & $196,304,331$ & $\begin{array}{c}216,239 \\
291\end{array}$ \\
\hline MinAD & $8.46 \mathrm{E}-04$ & 2.49E-05 & $2.33 \mathrm{E}-04$ & $5.34 \mathrm{E}-06$ & $5.02 \mathrm{E}-05$ & $2.06 \mathrm{E}-04$ & $2.93 \mathrm{E}-06$ & 3.37E-08 & 4.67E-05 & $1.56 \mathrm{E}-06$ & $2.55 \mathrm{E}-05$ & 4.17E-04 \\
\hline MinRD & $1.04 \mathrm{E}-02$ & $3.06 \mathrm{E}-04$ & $2.86 \mathrm{E}-03$ & $6.02 \mathrm{E}-05$ & 4.67E-04 & $1.85 \mathrm{E}-03$ & $1.98 \mathrm{E}-05$ & $2.53 \mathrm{E}-07$ & $3.62 \mathrm{E}-04$ & $2.77 \mathrm{E}-05$ & $3.70 \mathrm{E}-04$ & $5.47 \mathrm{E}-03$ \\
\hline \multirow[t]{2}{*}{ Return } & $8.2369 \%$ & $8.1571 \%$ & $8.1232 \%$ & $8.8661 \%$ & $10.7438 \%$ & $11.0784 \%$ & $14.7830 \%$ & $13.3495 \%$ & $12.9083 \%$ & $5.6250 \%$ & $6.8968 \%$ & $7.5800 \%$ \\
\hline & $(1.02 \mathrm{E}-03)$ & $(3.60 \mathrm{E}-03)$ & (3.10E-03) & $\left(883 \mathrm{~F}_{-} 03\right)$ & $(5.38 \mathrm{E}-03)$ & & & & & & $(5.29 \mathrm{E}-03)$ & $(5.72 \mathrm{E}-03)$ \\
\hline $\mathrm{T} 1, \mathrm{~T} 2, \mathrm{~T} 3$ & $12,13,14^{*}$ & $\begin{array}{c}153,228 \\
314\end{array}$ & $\begin{array}{c}203,292 \\
300\end{array}$ & $47,123,317$ & $\begin{array}{c}136,266, \\
315\end{array}$ & $\begin{array}{c}128,261 \\
337\end{array}$ & $45,115,342$ & $\begin{array}{c}241,287 \\
332\end{array}$ & $\begin{array}{c}140,327 \\
332\end{array}$ & $47,123,317$ & $186,234,337$ & $\begin{array}{c}180,283 \\
322\end{array}$ \\
\hline
\end{tabular}

* Bullet portfolio

** Barbell portfolio

*** Both bullet and barbell portfolios 
Table 19 - Continued

\begin{tabular}{|c|c|c|c|c|c|c|c|c|c|c|c|c|}
\hline \multicolumn{13}{|c|}{ Panel C: HJM Constant Decay Duration and Convexity } \\
\hline & \multicolumn{3}{|c|}{$\begin{array}{c}\text { Flat } \\
\text { (August, 1989) }\end{array}$} & \multicolumn{3}{|c|}{$\begin{array}{c}\text { Increasing } \\
\text { (January, 1985) }\end{array}$} & \multicolumn{3}{|c|}{$\begin{array}{l}\text { Decreasing } \\
(\text { May, 1981) }\end{array}$} & \multicolumn{3}{|c|}{$\begin{array}{c}\text { Humped } \\
\text { (October, 1986) }\end{array}$} \\
\hline & 1 year & 5 year & 10 year & 1 year & 5 year & 10 year & 1 year & 5 year & 10 year & 1 year & 5 year & 10 year \\
\hline$\%$ Successful & $99.02 \%$ & $95.10 \%$ & $100.00 \%$ & $98.04 \%$ & $94.12 \%$ & $99.02 \%$ & $98.04 \%$ & $98.04 \%$ & $99.02 \%$ & $95.10 \%$ & $93.14 \%$ & $98.04 \%$ \\
\hline Within 100 basis points & $100.00 \%$ & $100.00 \%$ & $100.00 \%$ & $100.00 \%$ & $100.00 \%$ & $100.00 \%$ & $98.04 \%$ & $100.00 \%$ & $100.00 \%$ & $97.06 \%$ & $100.00 \%$ & $98.04 \%$ \\
\hline Within 1 basis point & $95.10 \%{ }^{* * *}$ & $80.39 \%$ & $77.45 \%^{* * *}$ & $36.27 \%{ }^{* * *}$ & $87.25 \%{ }^{* * *}$ & $82.35 \%^{* * *}$ & $77.45 \%{ }^{* * *}$ & $87.25 \%{ }^{* * *}$ & $85.29 \%{ }^{* * *}$ & $46.08 \%{ }^{* * *}$ & $78.43 \%^{* * *}$ & $80.39 \%^{* * *}$ \\
\hline MaxAD & $1.12 \mathrm{E}-03$ & $6.75 \mathrm{E}-03$ & $1.05 \mathrm{E}-02$ & $3.92 \mathrm{E}-03$ & $3.97 \mathrm{E}-03$ & 7.67E-03 & $3.43 \mathrm{E}-02$ & $3.95 \mathrm{E}-03$ & $1.74 \mathrm{E}-02$ & $2.06 \mathrm{E}-02$ & $9.61 \mathrm{E}-03$ & $1.35 \mathrm{E}-02$ \\
\hline MaxRD & $1.37 \mathrm{E}-02$ & $8.27 \mathrm{E}-02$ & $1.29 \mathrm{E}-01$ & 4.43E-02 & $3.70 \mathrm{E}-02$ & $6.91 \mathrm{E}-02$ & $2.32 \mathrm{E}-01$ & $2.96 \mathrm{E}-02$ & $1.35 \mathrm{E}-01$ & $3.66 \mathrm{E}-01$ & $1.39 \mathrm{E}-01$ & $1.78 \mathrm{E}-01$ \\
\hline \multirow[t]{2}{*}{ Return } & $8.0408 \%$ & $7.4799 \%$ & $9.1935 \%$ & $9.2591 \%$ & $11.1362 \%$ & $11.8661 \%$ & $11.3486 \%$ & $12.9549 \%$ & $11.1678 \%$ & $3.5660 \%$ & $5.9384 \%$ & $8.9758 \%$ \\
\hline & $(2.72 \mathrm{E}-03)$ & $(6.73 \mathrm{E}-03)$ & $(1.38 \mathrm{E}-03)$ & $(4.67 \mathrm{E}-03)$ & (1.09E-03) & $(7.74 \mathrm{E}-03)$ & $(3.68 \mathrm{E}-02)$ & $(4.38 \mathrm{E}-03)$ & $(1.20 \mathrm{E}-02)$ & (3.67E-03) & $(6.88 \mathrm{E}-03)$ & $(1.44 \mathrm{E}-03)$ \\
\hline $\mathrm{T} 1, \mathrm{~T} 2, \mathrm{~T} 3$ & $100,222,339$ & $86,89,207$ & $191,219,306$ & $128,184,252$ & $164,277,289$ & $194,253,321$ & $49,136,266$ & $176,210,217$ & $210,261,338$ & $41,243,262$ & $187,297,346$ & $216,239,291$ \\
\hline $\operatorname{Min} A D$ & $1.61 \mathrm{E}-07$ & 4.91E-07 & 7.92E-08 & $4.85 \mathrm{E}-06$ & $5.02 \mathrm{E}-07$ & $8.53 \mathrm{E}-07$ & $1.57 \mathrm{E}-09$ & 3.79E-09 & $1.63 \mathrm{E}-08$ & $7.05 \mathrm{E}-08$ & 2.19E-07 & $9.12 \mathrm{E}-10$ \\
\hline MinRD & $1.98 \mathrm{E}-06$ & $6.02 \mathrm{E}-06$ & $9.72 \mathrm{E}-07$ & 4.37E-05 & 4.67E-06 & 7.69E-06 & $1.07 \mathrm{E}-08$ & 2.84E-08 & $1.26 \mathrm{E}-07$ & $1.25 \mathrm{E}-06$ & $3.18 \mathrm{E}-06$ & $1.20 \mathrm{E}-08$ \\
\hline \multirow[t]{2}{*}{ Return } & $8.1523 \%$ & $8.1545 \%$ & $8.1465 \%$ & $11.0995 \%$ & $10.7387 \%$ & $11.0989 \%$ & $14.7832 \%$ & $13.3495 \%$ & $12.9036 \%$ & $5.6248 \%$ & $6.8994 \%$ & $7.6217 \%$ \\
\hline & $(8.27 \mathrm{E}-07)$ & $(5.52 \mathrm{E}-06)$ & (6.49E-05) & $(6.96 \mathrm{E}-06)$ & (8.46E-07) & $(4.38 \mathrm{E}-05)$ & (2.19E-05) & $(5.54 \mathrm{E}-05)$ & $(2.66 \mathrm{E}-05)$ & (9.35E-07) & $(5.52 \mathrm{E}-06)$ & $(3.69 \mathrm{E}-05)$ \\
\hline $\mathrm{T} 1, \mathrm{~T} 2, \mathrm{~T} 3$ & $\begin{array}{c}12,120 \\
240^{* *}\end{array}$ & $12,144,240^{* *}$ & $205,292,325$ & $12,13,14^{*}$ & $100,271,323$ & $137,217,311$ & $15,41,49$ & $159,207,266$ & $123,279,293$ & $12,120,240$ & $67,89,222$ & $134,201,229$ \\
\hline
\end{tabular}


Under the passive portfolio management criterion, the number of portfolios that have returns within one basis point and 100 basis points of the target yield decreases with the duration and convexity matching strategy when Macaulay's risk measures are used. This result is in contradiction with our expectations. The reason for this finding may be due to the use of a convexity measure that does not take into account the exact nature of the volatility. Even though we are adjusting for a second-order term in the Taylor series expansion, this adjustment moves us further away. Therefore, the cash flows of the portfolios are not very close to those of the target.

Additionally, we observe unsuccessful bullet and barbell portfolios for five-year holding periods. For short holding periods, bullet and barbell portfolios do not always produce returns within one basis point of the target yield. Bullet portfolios produce the closest return to the target with duration matching. Therefore, we can say that time to maturity is an important determinant of duration for short holding periods but it is not necessarily for convexity in this HJM framework.

If we compare these results with the ones obtained with the duration matching strategy, we see that the convexity adjustment is important for active portfolio strategies when volatility is high. Under the passive portfolio management criterion and for relatively low volatility segments of the yield curve, however, the immunization performance of the portfolios worsens with the duration and convexity matching strategy when Macaulay's risk measures are used. Therefore, adjusting for Macaulay's duration and convexity does not lead to cash flows closer to those of the target. In this respect, risk managers who pursue passive portfolio management strategies are more prone to the risk of choosing inaccurate interest rate risk measures. Matching with both duration and convexity may not lead to better immunization performance for passive portfolio formation strategies if the risk measures used for immunization are not correct.

The results on the immunization performance of Fisher-Weil duration and convexity are shown in Table 19, Panel B. These results are similar to those of Macaulay's duration and convexity. Under the active portfolio management criterion, the main improvement in the performances of the portfolios is observed for short holding periods when there is high 
volatility. The number of successfully immunized portfolios increases by a range of $22 \%$ to $47 \%$ for one-year holding periods.

On the other hand, under the passive portfolio management criterion, the number of portfolios with returns within one basis point of the target yield decreases with the duration and convexity matching strategy. Therefore, the duration and convexity matching strategy with traditional risk measures does not lead to similar cash flows to that of the target. Similar to Macaulay's risk measures, bullet and barbell portfolios do not always have returns within one basis point of the target yield for one-year holding periods when Fisher-Weil risk measures are used. We observe unsuccessful bullet and barbell portfolios for medium holding periods.

Again these results suggest that risk managers who pursue passive portfolio management are more vulnerable to interest rate risk if they are using the incorrect risk measures. Traditional risk measures perform well with the duration and convexity matching strategy under the active portfolio management criterion, but they suffer significantly under the passive portfolio management criterion. Also, bullet portfolios have returns very close to that of the target with the duration matching strategy but this is not guaranteed with the duration and convexity matching strategy.

The performances of the portfolios that are matched with HJM duration and convexity measures under the constant decay volatility HJM framework are presented in Table 19, Panel C. The duration and convexity matching strategy provides similar or better immunization than the duration matching strategy for all holding periods under both active and passive portfolio management criteria. Under the active portfolio management criterion, the number of successful portfolios increases by up to $20 \%$ for long holding periods with the duration and convexity matching strategy compared to the duration matching strategy. For short to medium holding periods, when forward rate volatilities are high, the improvement is at most by $10 \%$ and mostly below $10 \%$. This result is different than what was found with traditional measures. When we use the HJM benchmark measures, the convexity adjustment seems to be more important for long holding periods rather than for the parts of the yield curve with high volatility. 
When the benchmark risk measures are used, the duration and convexity matching strategy generates cash flows closer to those of the target, which is not observed with traditional risk measures. The number of portfolios that have returns within one basis point of the target yield increases by a range of $30 \%$ to $79 \%$ with the duration and convexity matching strategy. In this respect, the immunization performance of portfolios that are matched with HJM duration and convexity improves significantly. This improvement is not observed with traditional risk measures. On the contrary, with traditional risk measures, the performance of portfolios deteriorates with the duration and convexity matching strategy when this passive portfolio management criterion is used.

With HJM risk measures, all bullet and barbell portfolios are not only successful but also have returns around one basis point of the target yield. More than $92 \%$ of the random portfolios are successful for all holding periods. Bullet portfolios are not necessarily the ones with the closest return to the target. Again, we observe that, for short holding periods, term to maturity is an important determinant of duration, but not necessarily for duration and convexity.

As shown in Table 20, the duration and convexity matching strategy did not reduce the deviation of returns of bullet and barbell portfolio when traditional risk measures are used. With HJM risk measures, however, the deviations of barbell portfolio returns reduce by 100 to 1000 times for short holding periods.

In these respects, it is apparent that under the constant decay volatility HJM framework, the duration and convexity matching strategy provides benefits for traditional risk measures only when active portfolio strategies are used. With passive portfolio management, the immunization performance of traditional risk measures deteriorates with the duration and convexity matching strategy. A risk manager is better off using only duration matching strategy with traditional risk measures under this framework. The immunization performances of the portfolios that are matched with HJM duration and convexity improve significantly under both active and passive portfolio management. In these respects, convexity adjustment has benefits only for active portfolio strategies with traditional risk measures. Also, these results suggest that using the correct risk measures is extremely important for risk managers. 
Table 20

\section{Performance of Duration and Convexity Matched Bullet and Barbell Portfolios under Constant Decay Volatility HJM Framework}

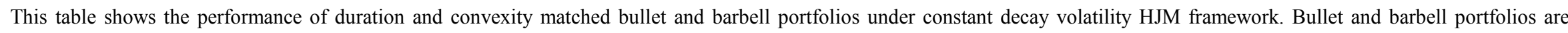

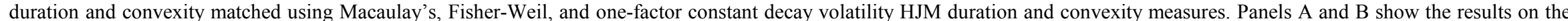

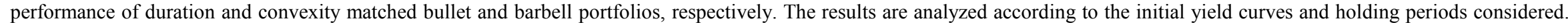

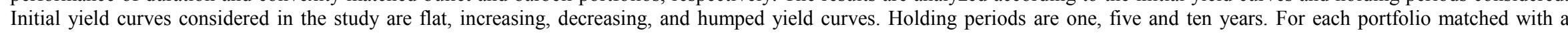

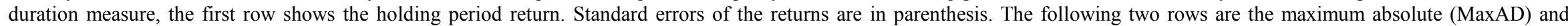
relative (MaxRD) deviations of the portfolio returns from the target return.

\begin{tabular}{|c|c|c|c|c|c|c|c|c|c|c|c|c|}
\hline \multicolumn{13}{|c|}{ Panel A: Bullet Portfolios } \\
\hline & \multicolumn{3}{|c|}{$\begin{array}{c}\text { Flat } \\
\text { (August, 1989) }\end{array}$} & \multicolumn{3}{|c|}{$\begin{array}{c}\text { Increasing } \\
(\text { January, 1985) }\end{array}$} & \multicolumn{3}{|c|}{$\begin{array}{c}\text { Decreasing } \\
(\text { May, 1981) }\end{array}$} & \multicolumn{3}{|c|}{$\begin{array}{c}\text { Humped } \\
\text { (October, 1986) }\end{array}$} \\
\hline & 1 year & 5 year & 10 year & 1 year & 5 year & 10 year & 1 year & 5 year & 10 year & 1 year & 5 year & 10 year \\
\hline \multirow[t]{2}{*}{ Macaulay } & $8.2369 \%$ & $8.2137 \%$ & $6.1767 \%$ & $8.8778 \%$ & $10.5238 \%$ & $11.1633 \%$ & $14.7871 \%$ & $13.7111 \%$ & $12.8856 \%$ & $5.6355 \%$ & $3.8278 \%$ & $7.7454 \%$ \\
\hline & (1.02E-03) & (4.41E-03) & $(3.07 \mathrm{E}-03)$ & (1.13E-03) & (8.36E-03) & $(2.53 \mathrm{E}-04)$ & (7.36E-04) & (3.16E-03) & (2.79E-04) & (1.21E-03) & (8.12E-03) & (1.04E-03) \\
\hline MaxAD & $8.46 \mathrm{E}-04$ & $5.91 \mathrm{E}-04$ & $1.97 \mathrm{E}-02$ & $1.11 \mathrm{E}-04$ & $2.15 \mathrm{E}-03$ & $6.44 \mathrm{E}-04$ & $3.88 \mathrm{E}-05$ & $3.62 \mathrm{E}-03$ & $1.80 \mathrm{E}-04$ & $1.07 \mathrm{E}-04$ & $3.07 \mathrm{E}-02$ & $1.24 \mathrm{E}-03$ \\
\hline MaxRD & $1.04 \mathrm{E}-02$ & $7.25 \mathrm{E}-03$ & $2.42 \mathrm{E}-01$ & $1.25 \mathrm{E}-03$ & $2.00 \mathrm{E}-02$ & $5.80 \mathrm{E}-03$ & $2.63 \mathrm{E}-04$ & $2.71 \mathrm{E}-02$ & $1.40 \mathrm{E}-03$ & $1.89 \mathrm{E}-03$ & $4.45 \mathrm{E}-01$ & $1.62 \mathrm{E}-02$ \\
\hline \multirow[t]{2}{*}{ Fisher-Weil } & & $8.2153 \%$ & & $8.8778 \%$ & $10.5273 \%$ & $11.2768 \%$ & $14.7871 \%$ & $13.5315 \%$ & $13.0058 \%$ & $5.6355 \%$ & $2.7147 \%$ & \\
\hline & (1.02E-03) & (4.41E-03) & $(6.74 \mathrm{E}-04)$ & (1.13E-03) & (8.39E-03) & (1.11E-03) & (7.36E-04) & (1.61E-03) & $(2.36 \mathrm{E}-03)$ & $(1.21 \mathrm{E}-03)$ & $(1.16 \mathrm{E}-02)$ & $(2.08 \mathrm{E}-03)$ \\
\hline MaxAD & $8.46 \mathrm{E}-04$ & $6.07 \mathrm{E}-04$ & $8.49 \mathrm{E}-04$ & $1.11 \mathrm{E}-04$ & $2.12 \mathrm{E}-03$ & $1.78 \mathrm{E}-03$ & $3.89 \mathrm{E}-05$ & $1.82 \mathrm{E}-03$ & $1.02 \mathrm{E}-03$ & $1.07 \mathrm{E}-04$ & 4.18E-02 & $2.12 \mathrm{E}-03$ \\
\hline MaxRD & $1.04 \mathrm{E}-02$ & $7.45 \mathrm{E}-03$ & $1.04 \mathrm{E}-02$ & $1.25 \mathrm{E}-03$ & $1.97 \mathrm{E}-02$ & $1.60 \mathrm{E}-02$ & $2.63 \mathrm{E}-04$ & $1.36 \mathrm{E}-02$ & $7.92 \mathrm{E}-03$ & $1.90 \mathrm{E}-03$ & $6.07 \mathrm{E}-01$ & $2.78 \mathrm{E}-02$ \\
\hline \multirow[t]{2}{*}{ HJM } & $8.1543 \%$ & $8.1540 \%$ & $8.1459 \%$ & $8.8667 \%$ & $10.3519 \%$ & $11.0995 \%$ & $14.7832 \%$ & $13.3528 \%$ & $12.9038 \%$ & $5.6248 \%$ & $6.5661 \%$ & $7.6289 \%$ \\
\hline & $(2.09 \mathrm{E}-04)$ & $(1.23 \mathrm{E}-03)$ & $(7.43 \mathrm{E}-05)$ & $(2.92 \mathrm{E}-04)$ & $(9.33 \mathrm{E}-03)$ & $(6.96 \mathrm{E}-06)$ & $(1.54 \mathrm{E}-04)$ & (1.07E-04) & $(1.00 \mathrm{E}-05)$ & $(2.61 \mathrm{E}-04)$ & $(5.77 \mathrm{E}-03)$ & $(6.48 \mathrm{E}-05)$ \\
\hline MaxAD & $1.95 \mathrm{E}-05$ & $5.53 \mathrm{E}-06$ & $6.72 \mathrm{E}-06$ & $1.62 \mathrm{E}-07$ & $3.87 \mathrm{E}-03$ & $4.85 \mathrm{E}-06$ & $3.46 \mathrm{E}-08$ & $3.35 \mathrm{E}-05$ & $1.91 \mathrm{E}-06$ & $1.36 \mathrm{E}-07$ & $3.33 \mathrm{E}-03$ & $7.18 \mathrm{E}-05$ \\
\hline MaxRD & $2.39 \mathrm{E}-04$ & $6.78 \mathrm{E}-05$ & $8.25 \mathrm{E}-05$ & $1.82 \mathrm{E}-06$ & $3.60 \mathrm{E}-02$ & $4.37 \mathrm{E}-05$ & $2.34 \mathrm{E}-07$ & $2.51 \mathrm{E}-04$ & $1.48 \mathrm{E}-05$ & $2.42 \mathrm{E}-06$ & $4.83 \mathrm{E}-02$ & $9.43 \mathrm{E}-04$ \\
\hline
\end{tabular}


Table 20 - Continued

\begin{tabular}{|c|c|c|c|c|c|c|c|c|c|c|c|c|}
\hline \multicolumn{13}{|c|}{ Panel B: Barbell Portfolios } \\
\hline Macaulay & $8.2437 \%$ & $8.2265 \%$ & $8.3062 \%$ & $8.8812 \%$ & $10.5238 \%$ & $11.4435 \%$ & $14.7884 \%$ & $13.7111 \%$ & $13.0346 \%$ & $5.6389 \%$ & $3.8278 \%$ & $7.9489 \%$ \\
\hline & $(2.48 \mathrm{E}-04)$ & $(1.86 \mathrm{E}-03)$ & $(3.49 \mathrm{E}-03)$ & (3.38E-04) & $(8.36 \mathrm{E}-03)$ & $(5.35 \mathrm{E}-03)$ & (1.94E-04) & (3.16E-03) & $(3.25 \mathrm{E}-03)$ & $(3.26 \mathrm{E}-04)$ & $(8.12 \mathrm{E}-02)$ & (5.10E-03) \\
\hline MaxAD & $9.13 \mathrm{E}-04$ & 7.20E-04 & $1.60 \mathrm{E}-03$ & $1.45 \mathrm{E}-04$ & $2.15 \mathrm{E}-03$ & $3.45 \mathrm{E}-03$ & $5.12 \mathrm{E}-05$ & $3.62 \mathrm{E}-03$ & $1.31 \mathrm{E}-03$ & $1.41 \mathrm{E}-04$ & $3.07 \mathrm{E}-02$ & $3.27 \mathrm{E}-03$ \\
\hline MaxRD & $1.12 \mathrm{E}-02$ & 8.82E-03 & $1.96 \mathrm{E}-02$ & $1.64 \mathrm{E}-03$ & $2.00 \mathrm{E}-02$ & $3.10 \mathrm{E}-02$ & $3.46 \mathrm{E}-04$ & $2.71 \mathrm{E}-02$ & $1.01 \mathrm{E}-02$ & $2.50 \mathrm{E}-03$ & $4.45 \mathrm{E}-01$ & $4.29 \mathrm{E}-02$ \\
\hline \multirow[t]{2}{*}{ Fisher-Weil } & $8.2441 \%$ & $8.2266 \%$ & $8.3043 \%$ & $8.8810 \%$ & $10.5273 \%$ & $11.4215 \%$ & $14.7882 \%$ & $13.5315 \%$ & $13.0160 \%$ & $5.6390 \%$ & $2.7147 \%$ & $7.9306 \%$ \\
\hline & (2.49E-04) & $(1.87 \mathrm{E}-03)$ & $(3.46 \mathrm{E}-03)$ & $(3.35 \mathrm{E}-04)$ & $(8.39 \mathrm{E}-03)$ & $(5.09 \mathrm{E}-03)$ & (1.89E-04) & (1.61E-03) & $(2.91 \mathrm{E}-03)$ & $(3.28 \mathrm{E}-04)$ & $(1.16 \mathrm{E}-02)$ & $(4.87 \mathrm{E}-03)$ \\
\hline MaxAD & $9.18 \mathrm{E}-04$ & $7.21 \mathrm{E}-04$ & $1.58 \mathrm{E}-03$ & $1.44 \mathrm{E}-04$ & $2.12 \mathrm{E}-03$ & $3.23 \mathrm{E}-03$ & $4.95 \mathrm{E}-05$ & $1.82 \mathrm{E}-03$ & $1.12 \mathrm{E}-03$ & $1.42 \mathrm{E}-04$ & $4.18 \mathrm{E}-02$ & $3.09 \mathrm{E}-03$ \\
\hline MaxRD & $1.13 \mathrm{E}-02$ & 8.84E-03 & $1.94 \mathrm{E}-02$ & $1.62 \mathrm{E}-03$ & $1.97 \mathrm{E}-02$ & $2.91 \mathrm{E}-02$ & $3.35 \mathrm{E}-04$ & $1.36 \mathrm{E}-02$ & 8.71E-03 & $2.52 \mathrm{E}-03$ & $6.07 \mathrm{E}-01$ & $4.05 \mathrm{E}-02$ \\
\hline \multirow[t]{2}{*}{ HJM } & $8.1523 \%$ & $8.1545 \%$ & $8.1464 \%$ & $8.8666 \%$ & $10.3519 \%$ & $11.0973 \%$ & $14.7832 \%$ & $13.3528 \%$ & $12.8993 \%$ & $5.6248 \%$ & $6.5661 \%$ & $7.6213 \%$ \\
\hline & $(8.27 \mathrm{E}-07)$ & $(5.52 \mathrm{E}-06)$ & $(8.34 \mathrm{E}-06)$ & $(8.90 \mathrm{E}-07)$ & $(9.33 \mathrm{E}-03)$ & $(1.27 \mathrm{E}-05)$ & $(6.48 \mathrm{E}-07)$ & $(1.07 \mathrm{E}-04)$ & $(8.78 \mathrm{E}-05)$ & $(9.35 \mathrm{E}-07)$ & $(5.77 \mathrm{E}-03)$ & $(1.02 \mathrm{E}-05)$ \\
\hline MaxAD & $1.61 \mathrm{E}-07$ & 4.91E-07 & 9.30E-07 & $7.45 \mathrm{E}-07$ & $3.87 \mathrm{E}-03$ & $1.63 \mathrm{E}-05$ & $6.74 \mathrm{E}-07$ & $3.35 \mathrm{E}-05$ & 4.30E-05 & 7.05E-08 & $3.33 \mathrm{E}-03$ & $4.22 \mathrm{E}-06$ \\
\hline MaxRD & $1.98 \mathrm{E}-06$ & $6.02 \mathrm{E}-06$ & $1.14 \mathrm{E}-05$ & $8.40 \mathrm{E}-06$ & $3.60 \mathrm{E}-02$ & $1.47 \mathrm{E}-04$ & $4.56 \mathrm{E}-06$ & $2.51 \mathrm{E}-04$ & $3.33 \mathrm{E}-04$ & $1.25 \mathrm{E}-06$ & 4.83E-02 & $5.54 \mathrm{E}-05$ \\
\hline
\end{tabular}


who pursue passive portfolio management. Since the exact nature of the volatility is not captured by traditional risk measures, adding the second order term to the Taylor series expansion of the bond price around its yield may lead us further away. For active portfolio strategies, traditional risk measures provide accurate immunization with the duration and convexity matching strategy. Both bullet and barbell portfolios produce very close returns to that of the target for short holding periods but they are not within one basis point of the target with traditional risk measures.

\subsection{Humped Volatility One-Factor HJM Framework}

The performances of traditional and HJM risk measures are compared under the humped volatility HJM framework and the results are presented in Table 21. In this table, Panel A and Panel B present the results using the Macaulay and Fisher-Weil duration and convexity measures, respectively. Panel $\mathrm{C}$ presents the results on the duration and convexity measures of humped volatility one-factor HJ M model.

With the duration matching strategy under the humped volatility HJM framework, we have seen that all duration measures are successful for all holding periods. In this respect, we cannot expect to find drastic improvements with the duration and convexity matching strategy. If we do see any improvement, we expect it to be for long holding periods, a result of the positive relation between convexity and time to maturity of a bond, and the high forward rate volatilities at the long end of the yield curve under this humped volatility structure ${ }^{55}$. We also expect to see an increase in the number of portfolios that have returns within one basis point of the target, since portfolios matched with duration and convexity should generate cash flows much like those of the target bond.

The results on the performances of Macaulay duration and convexity are presented in Table 21, Panel A. There is no significant improvement with the duration and convexity matching strategy under the active portfolio management criterion. Again almost all portfolios

\footnotetext{
${ }^{55}$ There is high volatility in the long holding periods, because the estimated volatility reduction factors are negative; thus volatilities increase with time to maturity. See Chapter 6, Section 6.3 for details.
} 


\section{Table 21}

\section{Performance of Duration and Convexity Matched Portfolios under Humped Volatility HJM Framework}

The performances of 102 duration and convexity matched portfolios are summarized. Two of the portfolios are bullet and barbell, and the rest are random portfolios. The benchmark portfolios are the ones that are matched according to the duration and convexity measures of the one factor HJM model with humped volatility. The other two duration measures are the Macaulay's and the Fisher-Weil duration and convexity measures. Panel A, B, and C present the results the performances of immunization portfolios matched by Macaulay's duration and convexity, Fisher-Weil duration and convexity, and the humped volatility one factor HJM duration and convexity, respectively. The results are analyzed according to the initial yield curves and holding periods. Four initial yield curves are considered: Flat (August 1989); increasing (January 1985); decreasing (May 1981); and humped (October 1986). The three holding periods are one-, five-, and ten-years. Three criteria are used to assess the immunization performances of the portfolios. According to one criterion, a portfolio is considered successful in immunization if the holding period return of the portfolio is greater than or equal to the yield of the target zero coupon bond minus 5 basis points. In Panels A and B, "\% Successful" ("\%Unsuccessful") gives the percentage of the portfolios that are successful (unsuccessful) in immunization according to the first criterion. The other two criteria assess the immunization performances by measuring the percentage of portfolios with returns within one and 100 basis points of the target yield. These are given in the next two rows. The maximum and minimum absolute (MaxAD and MinAD) and relative (MaxRD and MinRD) deviations from the target return are presented next. For each deviation, the returns of these portfolios, and the time to maturity of the bonds in the portfolios are given. Standard errors of the returns are in parentheses. Times to maturity are given in months, $(\mathrm{T} 1, \mathrm{~T} 2)$.

\begin{tabular}{|c|c|c|c|c|c|c|c|c|c|c|c|c|}
\hline \multicolumn{13}{|c|}{ Panel A: Macaulay’s Duration and Convexity } \\
\hline & \multicolumn{3}{|c|}{$\begin{array}{c}\text { Flat } \\
\text { (August, 1989) }\end{array}$} & \multicolumn{3}{|c|}{$\begin{array}{c}\text { Increasing } \\
\text { (January, 1985) }\end{array}$} & \multicolumn{3}{|c|}{$\begin{array}{c}\text { Decreasing } \\
(\text { May, 1981) }\end{array}$} & \multicolumn{3}{|c|}{$\begin{array}{c}\text { Humped } \\
\text { (October, 1986) }\end{array}$} \\
\hline & 1 year & 5 year & 10 year & 1 year & 5 year & 10 year & 1 year & 5 year & 10 year & 1 year & 5 year & 10 year \\
\hline \%Successful & $98.04 \%$ & $98.04 \%$ & $97.06 \%$ & $98.04 \%$ & $97.06 \%$ & $97.06 \%$ & $98.04 \%$ & $98.04 \%$ & $98.04 \%$ & $98.04 \%$ & $97.06 \%$ & $95.10 \%$ \\
\hline Within 100 basis points & $97.06 \%$ & $93.14 \%$ & $99.02 \%$ & $97.06 \%$ & $98.04 \%$ & $90.20 \%$ & $98.04 \%$ & $98.04 \%$ & $97.06 \%$ & $98.04 \%$ & $97.06 \%$ & $93.14 \%$ \\
\hline Within 1 basis point & $42.16 \%{ }^{* * *}$ & $50.98 \%^{* * *}$ & $84.31 \%{ }^{*}$ & $31.37 \%^{* * *}$ & $46.08 \%{ }^{* * *}$ & $52.94 \%$ & $47.06 \% \%^{* * *}$ & $84.31 \%^{* * *}$ & $86.57 \%^{* * *}$ & $37.25 \%{ }^{* * *}$ & $43.16 \%{ }^{* * *}$ & $70.59 \%{ }^{*}$ \\
\hline MaxAD & $3.62 \mathrm{E}-02$ & 7.01E-02 & $3.11 \mathrm{E}-02$ & $6.79 \mathrm{E}-02$ & $5.57 \mathrm{E}-02$ & $4.80 \mathrm{E}-02$ & $5.62 \mathrm{E}-02$ & $4.12 \mathrm{E}-02$ & $4.25 \mathrm{E}-02$ & $5.62 \mathrm{E}-02$ & $6.57 \mathrm{E}-02$ & $6.07 \mathrm{E}-02$ \\
\hline MaxRD & $4.44 \mathrm{E}-01$ & $8.60 \mathrm{E}-01$ & $3.82 \mathrm{E}-01$ & $7.65 \mathrm{E}-01$ & 5.19E-01 & $4.33 \mathrm{E}-01$ & $9.98 \mathrm{E}-01$ & $3.09 \mathrm{E}-01$ & $3.29 \mathrm{E}-01$ & $9.98 \mathrm{E}-01$ & $9.52 \mathrm{E}-01$ & $7.96 \mathrm{E}-01$ \\
\hline \multirow[t]{2}{*}{ Return } & $11.7754 \%$ & $1.1425 \%$ & $5.0335 \%$ & $15.6524 \%$ & $5.1685 \%$ & $6.2982 \%$ & $11.2398 \%$ & $9.2292 \%$ & $17.1496 \%$ & $11.2398 \%$ & $13.4671 \%$ & $1.5532 \%$ \\
\hline & $(1.03 \mathrm{E}-02)$ & $(5.66 \mathrm{E}-02)$ & $(3.10 \mathrm{E}-03)$ & $(6.08 \mathrm{E}-03)$ & $(2.36 \mathrm{E}-02)$ & $(1.42 \mathrm{E}-02)$ & $(8.02 \mathrm{E}-03)$ & $(4.51 \mathrm{E}-03)$ & $(4.32 \mathrm{E}-03)$ & $(8.02 \mathrm{E}-03)$ & $(8.25 \mathrm{E}-03)$ & $(5.95 \mathrm{E}-02)$ \\
\hline $\mathrm{T} 1, \mathrm{~T} 2, \mathrm{~T} 3$ & $\begin{array}{c}224,277 \\
329\end{array}$ & $\begin{array}{c}180,312 \\
319\end{array}$ & $\begin{array}{c}203,292 \\
300\end{array}$ & $\begin{array}{c}160,288 \\
345\end{array}$ & $\begin{array}{c}196,304 \\
331\end{array}$ & $\begin{array}{c}127,165 \\
324\end{array}$ & $\begin{array}{c}170,194 \\
266\end{array}$ & $\begin{array}{c}175,234 \\
306\end{array}$ & $\begin{array}{l}146,224 \\
323\end{array}$ & $\begin{array}{c}170,194 \\
266\end{array}$ & $182,236,277$ & $156,202,308$ \\
\hline MinAD & $9.69 \mathrm{E}-08$ & $1.62 \mathrm{E}-06$ & $2.70 \mathrm{E}-06$ & $1.84 \mathrm{E}-07$ & $1.22 \mathrm{E}-06$ & $1.43 \mathrm{E}-05$ & $1.79 \mathrm{E}-07$ & $2.79 \mathrm{E}-07$ & $3.13 \mathrm{E}-07$ & $1.79 \mathrm{E}-07$ & $4.05 \mathrm{E}-06$ & 8.77E-06 \\
\hline MinRD & $1.19 \mathrm{E}-06$ & $1.99 \mathrm{E}-05$ & $3.31 \mathrm{E}-05$ & $2.08 \mathrm{E}-06$ & $1.13 \mathrm{E}-05$ & $1.29 \mathrm{E}-04$ & $3.19 \mathrm{E}-06$ & $2.09 \mathrm{E}-06$ & $2.43 \mathrm{E}-06$ & $3.19 \mathrm{E}-06$ & $5.88 \mathrm{E}-05$ & $1.15 \mathrm{E}-04$ \\
\hline \multirow[t]{2}{*}{ Return } & $8.1523 \%$ & $8.1547 \%$ & $8.1463 \%$ & $8.8667 \%$ & $10.7387 \%$ & $11.1004 \%$ & $5.6249 \%$ & $13.3495 \%$ & $12.9036 \%$ & $5.6249 \%$ & $6.8990 \%$ & $7.6226 \%$ \\
\hline & (8.48E-06) & (6.40E-05) & $(3.48 \mathrm{E}-04)$ & $(1.20 \mathrm{E}-05)$ & (1.16E-04) & $(4.36 \mathrm{E}-04)$ & $(1.08 \mathrm{E}-05)$ & $(3.26 \mathrm{E}-05)$ & $(2.84 \mathrm{E}-04)$ & $(1.08 \mathrm{E}-05)$ & (1.08E-04) & $(2.23 \mathrm{E}-03)$ \\
\hline $\mathrm{T} 1, \mathrm{~T} 2, \mathrm{~T} 3$ & $12,13,14 *$ & $71,238,335$ & $\begin{array}{c}211,239 \\
244\end{array}$ & $12,13,14 *$ & $62,85,92$ & $\begin{array}{c}167,316 \\
317\end{array}$ & $12,13,14^{*}$ & $62,68,207$ & $\begin{array}{c}174,230 \\
245\end{array}$ & $12,13,14^{*}$ & $74,168,257$ & $216,262,292$ \\
\hline
\end{tabular}

\footnotetext{
* $\quad$ Bullet portfolio; ** Barbell portfolio; *** Both bullet and barbell portfolios
} 
Table 21 - Continued

\begin{tabular}{|c|c|c|c|c|c|c|c|c|c|c|c|c|}
\hline \multicolumn{13}{|c|}{ Panel B: Fisher-Weil Duration and Convexity } \\
\hline & \multicolumn{3}{|c|}{$\begin{array}{c}\text { Flat } \\
\text { (August, 1989) }\end{array}$} & \multicolumn{3}{|c|}{$\begin{array}{c}\text { Increasing } \\
\text { (January, 1985) }\end{array}$} & \multicolumn{3}{|c|}{$\begin{array}{l}\text { Decreasing } \\
\text { (May, 1981) }\end{array}$} & \multicolumn{3}{|c|}{$\begin{array}{c}\text { Humped } \\
\text { (October, 1986) }\end{array}$} \\
\hline & 1 year & 5 year & 10 year & 1 year & 5 year & 10 year & 1 year & 5 year & 10 year & 1 year & 5 year & 10 year \\
\hline$\%$ Successful & $99.02 \%$ & $98.04 \%$ & $97.06 \%$ & $99.02 \%$ & $98.04 \%$ & $97.06 \%$ & $99.02 \%$ & $99.02 \%$ & $98.04 \%$ & $99.02 \%$ & $98.04 \%$ & $96.08 \%$ \\
\hline Within 100 basis points & $99.02 \%$ & $98.04 \%$ & $100.00 \%$ & $98.04 \%$ & $97.06 \%$ & $98.04 \%$ & $97.06 \%$ & $100.00 \%$ & $100.00 \%$ & $97.06 \%$ & $99.02 \%$ & 100 \\
\hline Within 1 basis point & $44.12 \%^{* * *}$ & $49.02 \%^{* * *}$ & $82.35 \%{ }^{*}$ & $30.39 \%{ }^{* * *}$ & $41.18 \%{ }^{* * *}$ & $44.12 \%$ & $46.08 \%{ }^{* * *}$ & $50.98 \%{ }^{* * *}$ & $84.31 \%{ }^{* * *}$ & $26.47 \%^{* * *}$ & $41.18 \%{ }^{* * *}$ & $62.74 \%^{*}$ \\
\hline MaxAD & 7.74E-02 & $5.76 \mathrm{E}-02$ & $3.10 \mathrm{E}-02$ & $6.33 \mathrm{E}-02$ & $1.04 \mathrm{E}-02$ & $8.17 \mathrm{E}-03$ & $5.21 \mathrm{E}-02$ & $2.84 \mathrm{E}-03$ & $2.46 \mathrm{E}-02$ & $5.21 \mathrm{E}-02$ & $5.41 \mathrm{E}-02$ & $5.86 \mathrm{E}-02$ \\
\hline MaxRD & $9.50 \mathrm{E}-01$ & $7.06 \mathrm{E}-01$ & $3.80 \mathrm{E}-01$ & 7.14E-01 & $9.64 \mathrm{E}-02$ & $7.36 \mathrm{E}-02$ & $9.27 \mathrm{E}-01$ & 2.13E-02 & $1.91 \mathrm{E}-01$ & $9.27 \mathrm{E}-01$ & 7.84E-01 & 7.69E-01 \\
\hline \multirow[t]{2}{*}{ Return } & $0.4096 \%$ & $2.3995 \%$ & $5.0481 \%$ & $15.1940 \%$ & $11.7811 \%$ & $11.9164 \%$ & $10.8388 \%$ & $13.6336 \%$ & $10.4407 \%$ & $10.8388 \%$ & $1.4924 \%$ & $1.7620 \%$ \\
\hline & (2.64E-02) & $(1.53 \mathrm{E}-02)$ & $(2.29 \mathrm{E}-03)$ & $(8.70 \mathrm{E}-04)$ & $(1.27 \mathrm{E}-03)$ & & & & & (1.00E-03) & $(4.49 E-02)$ & $(2.30$ \\
\hline $\mathrm{T} 1, \mathrm{~T} 2, \mathrm{~T} 3$ & $\begin{array}{c}154,217 \\
266\end{array}$ & $\begin{array}{c}180,312 \\
319\end{array}$ & $\begin{array}{c}216,262 \\
292\end{array}$ & $\begin{array}{c}133,266 \\
279\end{array}$ & $85,321,328$ & $\begin{array}{c}145,161 \\
253\end{array}$ & $\begin{array}{c}153,222 \\
266\end{array}$ & $\begin{array}{c}168,277 \\
319\end{array}$ & $\begin{array}{c}124,244 \\
285\end{array}$ & $\begin{array}{c}153,222 \\
266\end{array}$ & $241,287,332$ & $\begin{array}{c}215,319 \\
325\end{array}$ \\
\hline $\operatorname{Min} A D$ & $9.69 \mathrm{E}-08$ & $1.61 \mathrm{E}-06$ & $3.93 \mathrm{E}-06$ & $1.84 \mathrm{E}-07$ & $1.09 \mathrm{E}-07$ & $8.02 \mathrm{E}-05$ & $1.79 \mathrm{E}-07$ & $9.68 \mathrm{E}-08$ & 4.96E-08 & $1.79 \mathrm{E}-07$ & 7.44E-06 & $3.86 \mathrm{E}-06$ \\
\hline MinRD & $1.19 \mathrm{E}-06$ & $1.98 \mathrm{E}-05$ & 4.83E-05 & $2.08 \mathrm{E}-06$ & $1.02 \mathrm{E}-06$ & 7.22E-04 & $3.19 \mathrm{E}-06$ & $7.25 \mathrm{E}-07$ & $3.84 \mathrm{E}-07$ & $3.19 \mathrm{E}-06$ & $1.08 \mathrm{E}-04$ & $5.06 \mathrm{E}-05$ \\
\hline \multirow[t]{2}{*}{ Return } & $8.1523 \%$ & $8.1544 \%$ & $8.1461 \%$ & $8.8667 \%$ & $10.7388 \%$ & $11.0910 \%$ & $5.6249 \%$ & $13.3495 \%$ & $12.9036 \%$ & $5.6249 \%$ & $6.8987 \%$ & $7.6221 \%$ \\
\hline & (8.47E-06) & $(6.94 \mathrm{E}-05)$ & $(3.29 \mathrm{E}-04)$ & $\left(120 F_{-} 05\right)$ & (1 10 Г Г & $(542504$ & (1) 08505 & & & & $(1.13 \mathrm{E}-0$ & $(1.25 \mathrm{E}-0$ \\
\hline $\mathrm{T} 1, \mathrm{~T} 2, \mathrm{~T} 3$ & $12,13,14^{*}$ & $71,143,196$ & $\begin{array}{c}211,239 \\
244\end{array}$ & $12,13,14^{*}$ & $78,142,226$ & $\begin{array}{c}181,188 \\
255\end{array}$ & $12,13,14^{*}$ & $62,68,207$ & $\begin{array}{c}181,273 \\
317 \\
\end{array}$ & $12,13,14^{*}$ & $81,89,277$ & $\begin{array}{c}231,292 \\
294 \\
\end{array}$ \\
\hline
\end{tabular}

* Bullet portfolio

** Barbell portfolio

*** Both bullet and barbell portfolios 
Table 21 - Continued

\begin{tabular}{|c|c|c|c|c|c|c|c|c|c|c|c|c|}
\hline \multicolumn{13}{|c|}{ Panel C: Humped Volatility HJM Duration and Convexity } \\
\hline & \multicolumn{3}{|c|}{$\begin{array}{c}\text { Flat } \\
\text { (August, 1989) }\end{array}$} & \multicolumn{3}{|c|}{$\begin{array}{c}\text { Increasing } \\
(\text { January, 1985) }\end{array}$} & \multicolumn{3}{|c|}{$\begin{array}{c}\text { Decreasing } \\
\text { (May, 1981) }\end{array}$} & \multicolumn{3}{|c|}{$\begin{array}{c}\text { Humped } \\
\text { (October, 1986) }\end{array}$} \\
\hline & 1 year & 5 year & 10 year & 1 year & 5 year & 10 year & 1 year & 5 year & 10 year & 1 year & 5 year & 10 year \\
\hline$\%$ Successful & $100.00 \%$ & $99.02 \%$ & $99.02 \%$ & $98.04 \%$ & $99.02 \%$ & $98.04 \%$ & $98.04 \%$ & $99.02 \%$ & $98.04 \%$ & $99.02 \%$ & $98.04 \%$ & $98.04 \%$ \\
\hline Within 100 basis points & $100.00 \%$ & $100.00 \%$ & $100.00 \%$ & $98.04 \%$ & $98.04 \%$ & $99.02 \%$ & $97.06 \%$ & $100.00 \%$ & $100.00 \%$ & $97.06 \%$ & $97.06 \%$ & $100.00 \%$ \\
\hline Within 1 basis point & $51.96 \%{ }^{* * *}$ & $62.74 \%^{* * *}$ & $92.16 \%{ }^{* * *}$ & $40.20 \%{ }^{* * *}$ & $53.92 \%^{* * *}$ & $90.20 \%{ }^{* *}$ & $50.00 \%{ }^{* * *}$ & $64.71 \%^{* * *}$ & $95.10 \%{ }^{* * *}$ & $40.20 \%{ }^{* * *}$ & $51.96 \%{ }^{* * *}$ & $86.27 \%{ }^{* *}$ \\
\hline MaxAD & $8.12 \mathrm{E}-03$ & $2.69 \mathrm{E}-03$ & $1.88 \mathrm{E}-03$ & $3.50 \mathrm{E}-02$ & $3.78 \mathrm{E}-02$ & 4.62E-02 & $2.41 \mathrm{E}-02$ & $2.70 \mathrm{E}-03$ & $1.15 \mathrm{E}-03$ & $2.41 \mathrm{E}-02$ & $2.43 \mathrm{E}-02$ & $9.10 \mathrm{E}-03$ \\
\hline MaxRD & $9.96 \mathrm{E}-02$ & $3.30 \mathrm{E}-02$ & $2.31 \mathrm{E}-02$ & $3.95 \mathrm{E}-01$ & $3.52 \mathrm{E}-01$ & $4.17 \mathrm{E}-01$ & $4.29 \mathrm{E}-01$ & $2.02 \mathrm{E}-02$ & $8.90 \mathrm{E}-03$ & $4.29 \mathrm{E}-01$ & $3.52 \mathrm{E}-01$ & $1.19 \mathrm{E}-01$ \\
\hline Return & $\begin{array}{c}7.3407 \% \\
(1.17 \mathrm{E}-03)\end{array}$ & $\begin{array}{c}8.4240 \% \\
(8.50 \mathrm{E}-05)\end{array}$ & $\begin{array}{c}7.9583 \% \\
(1.91 \mathrm{E}-03)\end{array}$ & $\begin{array}{l}12.3660 \% \\
(4.63 \mathrm{E}-03)\end{array}$ & $\begin{array}{c}6.9563 \% \\
(4.22 \mathrm{E}-03)\end{array}$ & $\begin{array}{l}15.7236 \% \\
(4.79 \mathrm{E}-03)\end{array}$ & $\begin{array}{c}8.0386 \% \\
(1.50 \mathrm{E}-03)\end{array}$ & $\begin{array}{r}13.0791 \% \\
(2.95 \mathrm{E}-03)\end{array}$ & $\begin{array}{l}12.7888 \% \\
(1.86 \mathrm{E}-03)\end{array}$ & $\begin{array}{c}8.0386 \% \\
(1.50 \mathrm{E}-03)\end{array}$ & $\begin{array}{c}4.4679 \% \\
(2.28 \mathrm{E}-03)\end{array}$ & $\begin{array}{c}6.7120 \% \\
(2.64 \mathrm{E}-03)\end{array}$ \\
\hline $\mathrm{T} 1, \mathrm{~T} 2, \mathrm{~T} 3$ & $152,306,315$ & $180,312,319$ & $207,292,307$ & $140,266,288$ & $195,216,277$ & $201,213,258$ & $152,306,315$ & $5182,210,276$ & $215,220,330$ & $152,306,315$ & $195,216,277$ & $264,300,335$ \\
\hline $\operatorname{Min} A D$ & $5.24 \mathrm{E}-10$ & $1.20 \mathrm{E}-06$ & $2.53 \mathrm{E}-06$ & $1.51 \mathrm{E}-09$ & $6.01 \mathrm{E}-07$ & $5.89 \mathrm{E}-06$ & $1.27 \mathrm{E}-09$ & $5.37 \mathrm{E}-07$ & $7.29 \mathrm{E}-07$ & $1.27 \mathrm{E}-09$ & $9.71 \mathrm{E}-07$ & $5.11 \mathrm{E}-06$ \\
\hline MinRD & $6.43 \mathrm{E}-09$ & $1.47 \mathrm{E}-05$ & $3.10 \mathrm{E}-05$ & $1.71 \mathrm{E}-08$ & $5.60 \mathrm{E}-06$ & $5.31 \mathrm{E}-05$ & $2.26 \mathrm{E}-08$ & $4.02 \mathrm{E}-06$ & $5.65 \mathrm{E}-06$ & $2.26 \mathrm{E}-08$ & $1.41 \mathrm{E}-05$ & $6.70 \mathrm{E}-05$ \\
\hline Return & $\begin{array}{c}8.1523 \% \\
(7.37 \mathrm{E}-06)\end{array}$ & $\begin{array}{c}8.1547 \% \\
(4.47 \mathrm{E}-08)\end{array}$ & $\begin{array}{c}8.1463 \% \\
(2.40 \mathrm{E}-06)\end{array}$ & $\begin{array}{c}8.8667 \% \\
(1.06 \mathrm{E}-05)\end{array}$ & $\begin{array}{r}10.7389 \% \\
(5.28 \mathrm{E}-07)\end{array}$ & $\begin{array}{l}11.0984 \% \\
(2.76 \mathrm{E}-05)\end{array}$ & $\begin{array}{c}5.6248 \% \\
(9.12 \mathrm{E}-06)\end{array}$ & $\begin{array}{r}13.3494 \% \\
(1.51 \mathrm{E}-07)\end{array}$ & $\begin{array}{l}12.9037 \% \\
(1.28 \mathrm{E}-05)\end{array}$ & $\begin{array}{l}5.6248 \% \\
(9.12 \mathrm{E}-06)\end{array}$ & $\begin{array}{c}6.8995 \% \\
(2.83 \mathrm{E}-08)\end{array}$ & $\begin{array}{c}7.6222 \% \\
(1.83 \mathrm{E}-04)\end{array}$ \\
\hline $\mathrm{T} 1, \mathrm{~T} 2, \mathrm{~T} 3$ & $12,13,14^{*}$ & $71,143,196$ & $122,140,145$ & $12,13,14^{*}$ & $70,229,337$ & $122,140,145$ & $12,13,14^{*}$ & $71,143,196$ & $172,178,200$ & $12,13,14^{*}$ & $71,143,196$ & $207,292,307$ \\
\hline
\end{tabular}


generate returns greater than or equal to that of the target bond. The number of successfully immunized portfolios is slightly less with the duration and convexity matching strategy, but the difference is not significant. Under the passive portfolio management, the number of portfolios that have returns within one basis point of the target yield is also slightly less with the duration-convexity matching. These results suggest that the convexity adjustment did not add any value, and in fact on the contrary lead to slight deterioration when Macaulay's measures are used. This shows that unless we know the correct risk measures, adjusting for convexity does not always lead to better performance than the duration matching strategy.

Similar to the results of the duration matching strategy, bullet and barbell portfolios have returns within one basis point of the target yield for one- and five-year holding periods. All bullet and barbell portfolios and more than 95\% of random portfolios are successful for all holding periods under the active portfolio management criterion. Bullet portfolios have the closest returns to the target yield for one-year holding periods. In these respects, again the duration and convexity matching strategy did not add additional value to the performance of portfolios under the humped volatility HJM framework.

Table 21, Panel B presents the results on the performance of Fisher-Weil duration and convexity under the humped volatility HJM framework. The results are similar to those found with Macaulay's risk measures. The duration and convexity matching strategy did not enhance the immunization performance. The performances of portfolios are similar to the ones matched only with duration. The number of duration and convexity matched portfolios that are successful under the active portfolio management criterion and the portfolios with returns within one basis point of the target yield are slightly less with the duration and convexity matching strategy. Bullet and barbell portfolios have returns within one basis point of the target yield for one- and five-year holding periods. Bullet portfolios have the closest returns to the target yield for one-year holding periods. The duration and convexity matching strategy did not improve the immunization performance of portfolios that are matched with the FisherWeil risk measures under the humped volatility HJM framework.

Table 21, Panel C presents the performance of the HJM duration and convexity measures for the humped volatility one-factor HJM model. Similar to the results related to the 
Macaulay and Fisher-Weil measures, the duration and convexity matching strategy did not improve the immunization performance of the portfolios. The immunization performance is almost identical to that with the duration matching strategy. Compared to the performances of traditional risk measures, however, we do not observe any deterioration of immunization performance with the HJM risk measures with the duration and convexity matching strategy. Again this result supports previous evidence that unless the appropriate risk measures are used, one cannot be assured of an increase in the immunization performance with the duration and convexity matching strategy compared to the duration matching strategy. Therefore, especially under the passive portfolio management criterion, selection of the risk measure is more important than the immunization strategy.

As presented in Table 22, the holding period returns of bullet and barbell portfolios are very close to the target yield especially for short holding periods. Also the duration and convexity matching strategy did not reduce the deviation of the portfolio returns from the target yield compared to the duration matching strategy.

In these respects, we can easily say that under the humped volatility HJM framework, one can use duration-matched portfolios where the traditional measures or the HJM measures are used to match for duration. The duration matching strategy is sufficient for immunization under this HJM framework. The duration and convexity matching strategy did not add significant value to the immunization process. In fact, it led to a slight deterioration of the immunization performance when traditional risk measures are used. This deterioration is not observed with HJM risk measures. Bullet and barbell portfolios can be preferred to other alternatives for short holding period since they are immunized under the active portfolio management criterion, and they have returns within one basis point of the target yield.

\subsection{Discussion on the Performances of Duration and Convexity Matched Portfolios}

The results on the performance of the duration and convexity matching strategy suggest that adjusting for convexity improves the immunization performance of traditional risk measures drastically when active portfolio strategies are pursued at the segments of the yield curve where forward rate volatilities are high. This drastic improvement is not observed 
for HJM risk measures under the active portfolio management criterion. With the duration and convexity matching strategy, improvement in the performance of HJM risk measures is not necessarily at the high volatility segments of the yield curve, but rather for long holding periods. In this respect, with HJM risk measures the convexity adjustment is important mainly for long holding periods. This may be due to the positive relation between convexity and time to maturity. Compared to the duration matching strategy, with traditional duration and convexity measures the performances of immunization portfolios slightly deteriorate at the segments of the yield curve where forward rate volatilities are low. Also, we observe deterioration in the immunization performance of traditional risk measures under constant decay and humped volatility HJM models under the passive portfolio management criterion. In this respect, for risk managers who pursue passive portfolio management, using the appropriate risk measures are extremely important. Using duration and convexity matched portfolios instead of duration matched portfolios does not guarantee improvement in the immunization performance with traditional risk measures. Under the active portfolio management criterion, however, the duration and convexity matching strategy improves the performance of traditional risk measures.

This evidence suggests that asset-liability managers who pursue active portfolio management strategies can still prefer traditional duration and convexity measures to HJM risk measures due to the implementation related costs of HJM risk measures. This decision will lead to generally around $10 \%$ to $15 \%$ under-performance. In fact in the real world, assetliability managers can even end up finding that at times traditional risk measures immunize better than HJM risk measures. This result can occur because this study is a simulation in which the underlying interest rate process is controlled and the benchmarks are HJM risk measures. Therefore, there are no model, calibration or estimation problems related to the HJM measures. In the real world, however, estimation and calibration problems may deteriorate the performance of HJM risk measures. In this case, traditional risk measures could end up providing better immunization performance than their HJM counterparts. Perhaps this also explains why risk managers still use traditional risk measures for more than 30 years. 


\section{Table 22}

\section{Performance of Duration and Convexity Matched Bullet and Barbell Portfolios under Humped Volatility HJM Framework}

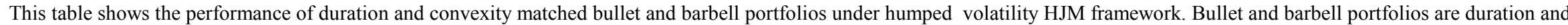

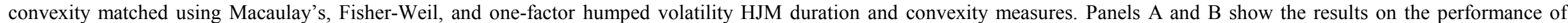

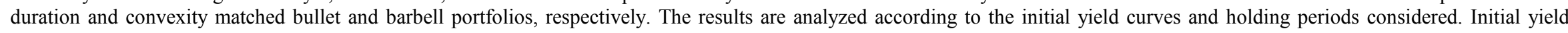

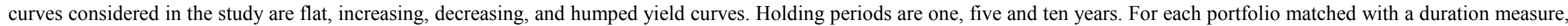

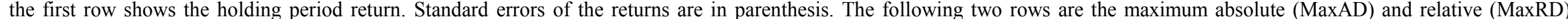
deviations of the portfolio returns from the target return.

\begin{tabular}{|c|c|c|c|c|c|c|c|c|c|c|c|c|}
\hline \multicolumn{13}{|c|}{ Panel A: Bullet Portfolios } \\
\hline & \multicolumn{3}{|c|}{$\begin{array}{c}\text { Flat } \\
\text { (August, 1989) }\end{array}$} & \multicolumn{3}{|c|}{$\begin{array}{c}\text { Increasing } \\
\text { (January, 1985) }\end{array}$} & \multicolumn{3}{|c|}{$\begin{array}{l}\text { Decreasing } \\
(\text { May, 1981) }\end{array}$} & \multicolumn{3}{|c|}{$\begin{array}{c}\text { Humped } \\
\text { (October, 1986) }\end{array}$} \\
\hline & 1 year & 5 year & 10 year & 1 year & 5 year & 10 year & 1 year & 5 year & 10 year & 1 year & 5 year & 10 year \\
\hline \multirow[t]{2}{*}{ Macaulay } & $8.1523 \%$ & $8.1335 \%$ & $8.1417 \%$ & $8.8667 \%$ & $10.7194 \%$ & $10.6923 \%$ & $5.6249 \%$ & $13.3368 \%$ & $12.9316 \%$ & $5.6249 \%$ & $6.8717 \%$ & $7.6254 \%$ \\
\hline & $(8.48 \mathrm{E}-06)$ & $(3.88 \mathrm{E}-04)$ & $(7.07 \mathrm{E}-04)$ & $(1.20 \mathrm{E}-05)$ & $(5.32 \mathrm{E}-04)$ & (7.01E-04) & $(1.08 \mathrm{E}-05)$ & $(4.31 \mathrm{E}-04)$ & $(4.60 \mathrm{E}-04)$ & $(1.08 \mathrm{E}-05)$ & $(5.28 \mathrm{E}-04)$ & $(4.67 \mathrm{E}-04)$ \\
\hline MaxAD & $9.69 \mathrm{E}-08$ & $2.11 \mathrm{E}-04$ & $4.81 \mathrm{E}-05$ & $1.84 \mathrm{E}-07$ & $1.93 \mathrm{E}-04$ & 4.07E-03 & $1.79 \mathrm{E}-07$ & $1.27 \mathrm{E}-04$ & $2.80 \mathrm{E}-04$ & $1.79 \mathrm{E}-07$ & $2.77 \mathrm{E}-04$ & $3.66 \mathrm{E}-05$ \\
\hline MaxRD & $1.19 \mathrm{E}-06$ & $2.58 \mathrm{E}-03$ & $5.91 \mathrm{E}-04$ & $2.08 \mathrm{E}-06$ & $1.80 \mathrm{E}-03$ & $3.66 \mathrm{E}-02$ & $3.19 \mathrm{E}-06$ & $9.51 \mathrm{E}-04$ & $2.17 \mathrm{E}-03$ & $3.19 \mathrm{E}-06$ & $4.02 \mathrm{E}-03$ & $4.80 \mathrm{E}-04$ \\
\hline \multirow[t]{2}{*}{ Fisher-Weil } & $8.1523 \%$ & $8.1335 \%$ & $8.1410 \%$ & $8.8667 \%$ & $10.7200 \%$ & $10.6935 \%$ & $5.6249 \%$ & $13.3364 \%$ & $12.8972 \%$ & $5.6249 \%$ & $6.8718 \%$ & $7.6197 \%$ \\
\hline & $(8.47 \mathrm{E}-06)$ & (3.88E-04) & $(7.21 \mathrm{E}-04)$ & (1.20E-05) & $(5.23 \mathrm{E}-04)$ & (6.82E-04) & (1.08E-05) & $(4.35 \mathrm{E}-04)$ & $(6.65 \mathrm{E}-05)$ & (1.08E-05) & $(5.26 \mathrm{E}-04)$ & $(4.81 \mathrm{E}-04)$ \\
\hline MaxAD & $9.69 \mathrm{E}-08$ & $2.10 \mathrm{E}-04$ & $5.50 \mathrm{E}-05$ & $1.84 \mathrm{E}-07$ & $1.88 \mathrm{E}-04$ & $4.05 \mathrm{E}-03$ & $1.79 \mathrm{E}-07$ & $1.31 \mathrm{E}-04$ & $6.47 \mathrm{E}-05$ & $1.79 \mathrm{E}-07$ & $2.76 \mathrm{E}-04$ & $2.00 \mathrm{E}-05$ \\
\hline MaxRD & $1.19 \mathrm{E}-06$ & $2.58 \mathrm{E}-03$ & $6.75 \mathrm{E}-04$ & $2.08 \mathrm{E}-06$ & $1.75 \mathrm{E}-03$ & $3.65 \mathrm{E}-02$ & $3.19 \mathrm{E}-06$ & $9.78 \mathrm{E}-04$ & $5.01 \mathrm{E}-04$ & $3.19 \mathrm{E}-06$ & $4.00 \mathrm{E}-03$ & $2.62 \mathrm{E}-04$ \\
\hline \multirow[t]{2}{*}{ HJM } & $8.1523 \%$ & $8.1384 \%$ & $8.1353 \%$ & $8.8667 \%$ & $10.7236 \%$ & $10.9781 \%$ & $5.6248 \%$ & $13.3380 \%$ & $12.9027 \%$ & $5.6248 \%$ & $6.8783 \%$ & $7.5945 \%$ \\
\hline & $(7.37 \mathrm{E}-06)$ & $(2.83 \mathrm{E}-04)$ & (8.87E-04) & $(1.06 \mathrm{E}-05)$ & $(4.59 \mathrm{E}-04)$ & (8.71E-04) & (9.12E-06) & (4.19E-04) & $(2.36 \mathrm{E}-06)$ & (9.12E-06) & (3.68E-04) & (7.28E-04) \\
\hline MaxAD & $5.24 \mathrm{E}-10$ & $1.62 \mathrm{E}-04$ & $1.12 \mathrm{E}-04$ & $1.51 \mathrm{E}-09$ & $1.52 \mathrm{E}-04$ & $1.21 \mathrm{E}-03$ & $1.27 \mathrm{E}-09$ & $1.15 \mathrm{E}-04$ & $9.19 \mathrm{E}-06$ & $1.27 \mathrm{E}-09$ & $2.11 \mathrm{E}-04$ & $2.72 \mathrm{E}-04$ \\
\hline MaxRD & $6.43 \mathrm{E}-09$ & $1.99 \mathrm{E}-03$ & $1.37 \mathrm{E}-03$ & $1.71 \mathrm{E}-08$ & $1.41 \mathrm{E}-03$ & $1.09 \mathrm{E}-02$ & $2.26 \mathrm{E}-08$ & $8.60 \mathrm{E}-04$ & $7.12 \mathrm{E}-05$ & $2.26 \mathrm{E}-08$ & $3.06 \mathrm{E}-03$ & $3.57 \mathrm{E}-03$ \\
\hline
\end{tabular}


Table 22 - Continued

\begin{tabular}{|c|c|c|c|c|c|c|c|c|c|c|c|c|}
\hline \multicolumn{13}{|c|}{ Panel B: Barbell Portfolios } \\
\hline \multirow[t]{2}{*}{ Macaulay } & $8.1520 \%$ & $8.1335 \%$ & $8.1309 \%$ & $8.8659 \%$ & $10.7194 \%$ & $11.0233 \%$ & $5.6239 \%$ & $13.3368 \%$ & $12.9021 \%$ & $5.6239 \%$ & $6.8717 \%$ & $7.5535 \%$ \\
\hline & (9.87E-07) & $(3.88 \mathrm{E}-04)$ & (1.45E-04) & $(1.54 \mathrm{E}-06)$ & (5.32E-04) & (3.04E-04) & $(1.47 \mathrm{E}-06)$ & (4.31E-04) & (2.13E-04) & $(1.47 \mathrm{E}-06)$ & $(5.28 \mathrm{E}-04)$ & (2.97E-04) \\
\hline MaxAD & $3.22 \mathrm{E}-06$ & $2.11 \mathrm{E}-04$ & $1.56 \mathrm{E}-04$ & $7.98 \mathrm{E}-06$ & $1.93 \mathrm{E}-04$ & $7.57 \mathrm{E}-04$ & $9.15 \mathrm{E}-06$ & $1.27 \mathrm{E}-04$ & $1.56 \mathrm{E}-05$ & $9.15 \mathrm{E}-06$ & $2.77 \mathrm{E}-04$ & $6.83 \mathrm{E}-04$ \\
\hline MaxRD & $3.95 \mathrm{E}-05$ & $2.58 \mathrm{E}-03$ & $1.91 \mathrm{E}-03$ & $9.00 \mathrm{E}-05$ & $1.80 \mathrm{E}-03$ & $6.82 \mathrm{E}-03$ & $1.63 \mathrm{E}-04$ & $9.51 \mathrm{E}-04$ & $1.21 \mathrm{E}-04$ & $1.63 \mathrm{E}-04$ & $4.02 \mathrm{E}-03$ & $8.96 \mathrm{E}-03$ \\
\hline \multirow[t]{2}{*}{ Fisher-Weil } & $8.1520 \%$ & $8.1335 \%$ & $8.1316 \%$ & $8.8659 \%$ & $10.7200 \%$ & $11.0384 \%$ & $5.6239 \%$ & $13.3364 \%$ & $12.8969 \%$ & $5.6239 \%$ & $6.8718 \%$ & $7.5742 \%$ \\
\hline & (1.01E-06) & $(3.88 \mathrm{E}-04)$ & (1.37E-04) & $(1.44 \mathrm{E}-06)$ & $(5.23 \mathrm{E}-04)$ & (2.18E-04) & $(1.53 \mathrm{E}-06)$ & (4.35E-04) & $(6.28 \mathrm{E}-05)$ & $(1.53 \mathrm{E}-06)$ & $(5.26 \mathrm{E}-04)$ & $(2.32 \mathrm{E}-04)$ \\
\hline MaxAD & $3.21 \mathrm{E}-06$ & $2.10 \mathrm{E}-04$ & $1.49 \mathrm{E}-04$ & 8.09E-06 & $1.88 \mathrm{E}-04$ & $6.05 \mathrm{E}-04$ & $9.01 \mathrm{E}-06$ & $1.31 \mathrm{E}-04$ & $6.68 \mathrm{E}-05$ & $9.01 \mathrm{E}-06$ & $2.76 \mathrm{E}-04$ & $4.75 \mathrm{E}-04$ \\
\hline MaxRD & $3.94 \mathrm{E}-05$ & $2.58 \mathrm{E}-03$ & $1.83 \mathrm{E}-03$ & $9.12 \mathrm{E}-05$ & $1.75 \mathrm{E}-03$ & $5.45 \mathrm{E}-03$ & $1.60 \mathrm{E}-04$ & $9.78 \mathrm{E}-04$ & $5.18 \mathrm{E}-04$ & $1.60 \mathrm{E}-04$ & $4.00 \mathrm{E}-03$ & $6.24 \mathrm{E}-03$ \\
\hline \multirow[t]{2}{*}{ HJM } & $8.1523 \%$ & $8.1384 \%$ & $8.1389 \%$ & $8.8665 \%$ & $10.7236 \%$ & $11.0782 \%$ & $5.6247 \%$ & $13.3380 \%$ & $12.8997 \%$ & $5.6247 \%$ & $6.8783 \%$ & $7.6038 \%$ \\
\hline & $(1.41 \mathrm{E}-08)$ & $(2.83 \mathrm{E}-04)$ & $(1.04 \mathrm{E}-07)$ & $(4.24 \mathrm{E}-08)$ & $(4.59 \mathrm{E}-04)$ & $(5.98 \mathrm{E}-07)$ & $(5.20 \mathrm{E}-08)$ & $(4.19 \mathrm{E}-04)$ & $(2.23 \mathrm{E}-06)$ & $(5.20 \mathrm{E}-08)$ & $(3.68 \mathrm{E}-04)$ & $(2.65 \mathrm{E}-08)$ \\
\hline MaxAD & $5.98 \mathrm{E}-07$ & $1.62 \mathrm{E}-04$ & $7.60 \mathrm{E}-05$ & $1.36 \mathrm{E}-06$ & $1.52 \mathrm{E}-04$ & $2.08 \mathrm{E}-04$ & $1.34 \mathrm{E}-06$ & $1.15 \mathrm{E}-04$ & 3.89E-05 & $1.34 \mathrm{E}-06$ & $2.11 \mathrm{E}-04$ & $1.80 \mathrm{E}-04$ \\
\hline MaxRD & 7.33E-06 & $1.99 \mathrm{E}-03$ & $9.33 \mathrm{E}-04$ & $1.53 \mathrm{E}-05$ & $1.41 \mathrm{E}-03$ & $1.87 \mathrm{E}-03$ & $2.39 \mathrm{E}-05$ & $8.60 \mathrm{E}-04$ & $3.02 \mathrm{E}-04$ & $2.39 \mathrm{E}-05$ & $3.06 \mathrm{E}-03$ & $2.36 \mathrm{E}-03$ \\
\hline
\end{tabular}


For risk managers who follow passive portfolio management, however, it is extremely important to determine appropriate risk measures. Contrary to expectations, duration and convexity matching strategy can lead to a slight deterioration of immunization performance when traditional risk measures are used. In this respect, if an asset-liability manager pursues passive strategies, the duration matching strategy can be as good as the duration and convexity matching strategy. The most important step should be deciding on the risk measures rather than the immunization strategies.

When the duration and convexity matching strategy is used, both bullet and barbell portfolios produce returns very close to that of the target for short holding periods. In this respect, bullet and barbell portfolios can be preferred to other alternatives for short holding periods. For medium to long holding periods, however, we are not able to favor one portfolio formation strategy over another. 


\section{CHAPTER 9}

\section{PERFORMANCE OF MINIMUM M-SQUARE PORTFOLIOS}

The M-square measure can be interpreted as the time-to-payment variance around duration at the coupon payment dates of the coupon bond. Minimizing the M-square of a duration matched portfolio reduces its variance. Therefore, a minimum M-square portfolio will not be affected much from the shifts that could not be immunized by the duration measure alone. Traditional duration measures are successful in immunization when the yield curve shifts are parallel. If we minimize the M-squares of these duration-matched portfolios,

then they should be immune to non-parallel shifts in the yield curve as well. This result, however, is not guaranteed. If the M-square of a duration-matched portfolio were zero, then a duration-matching strategy would be sufficient for immunization. Minimizing M-square, on the other hand, does not necessarily make M-square zero. Therefore, even minimum M-square portfolios can still be exposed to interest rate risk when yield curve shifts are non-parallel. The exposure, however, should be less than that of portfolios with higher M-square values.

In this study, different risk measures are considered. Two of these measures are the Macaulay and Fisher-Weil risk measures developed to immunize for parallel shifts in the yield curve. The other three are developed for the selected three one-factor HJM models. The forward rates of the selected HJM models have exponentially decaying, constant decay, and humped volatilities. Minimizing the M-squares of the portfolios that are matched with specific risk measures will reduce the effects of the shifts in the yield curves that cannot be immunized by the selected risk measures.

When forward rates are simulated according to a specific HJM model, the risk measures corresponding to that model provide the benchmark. In this respect, the HJM risk measures should be sufficient to immunize against yield curve shifts that arise with the selected HJM model. If the volatility is high, then convexity should capture the nonlinear relation between the bond price and the yield change. Therefore, in high volatility cases, duration and convexity matching strategy with the selected HJM risk measures should be 
sufficient for immunization. Therefore, minimizing M-square should not lead to any significant improvement in the immunization performance of the portfolios formed using HJM risk measures. We expect, however, to observe improvement in the immunization performance of the portfolios formed by the traditional risk measures. Traditional risk measures immunize only against parallel shifts. Therefore, under the exponential decay, constant decay, and humped volatility HJM frameworks, the immunization performance of the portfolios formed by traditional risk measures can be improved by minimizing their Msquares. In these respects, we analyze the performances of minimum M-square portfolios according to the HJM models underlying the forward rate evolution.

\subsection{Constant Volatility One-Factor HJM Framework}

Under this framework, yield curve shifts are parallel. Therefore, we do not expect to observe improvement in the immunization performance of portfolios in which we use the Fisher-Weil duration measure. We expect to observe improvement in the immunization performance of the portfolios matched with Macaulay's duration. Macaulay's duration assumes a flat yield curve. Therefore, for non-flat yield curves, the immunization performance of portfolios formed by Macaulay's duration can be improved by minimizing their M-squares.

The results on the performances of duration-matched minimum M-square portfolios are presented in Table 23, Panel A. We consider three criteria to test the immunization performance of the portfolios. According to the criterion that corresponds to active portfolio strategies, a portfolio is considered to be successfully immunized if its holding period return is greater than or equal to the target yield minus five basis points. The other two criteria correspond to passive portfolio management. According to one of these criteria, we consider a portfolio successfully immunized if its holding period return is within one basis point of the target. According to the other criteria, the portfolio is successfully immunized if its holding period return is within 100 basis points of the target.

Under the active portfolio management criterion, all minimum M-square portfolios are successful whether they are matched with Macaulay's or Fisher-Weil duration. These results are as expected. Under the constant volatility HJM framework, yield curve shifts are 


\section{Table 23}

\section{Performance of Minimum M-Square Portfolios under Constant Volatility HJM Framework}

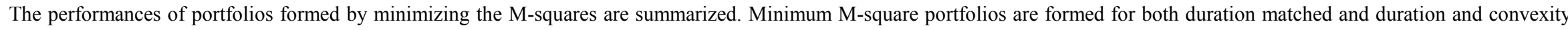

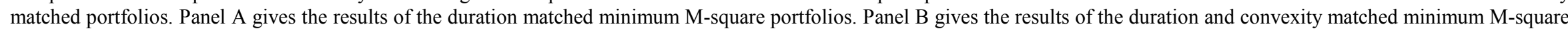

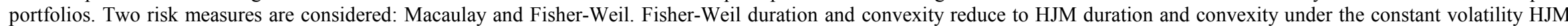

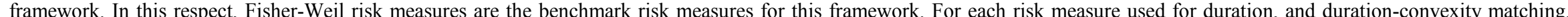

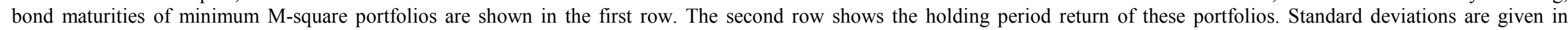
parentheses. The next two rows show the absolute and relative deviation of the holding period returns from the target yield.

Panel A

Duration Matched Minimum M-Square Portfolios

\begin{tabular}{|c|c|c|c|c|c|c|c|c|c|c|c|c|}
\hline \multicolumn{13}{|c|}{ Duration Matched Minimum M-Square Portfolios } \\
\hline & \multicolumn{3}{|c|}{$\begin{array}{c}\text { Flat } \\
\text { (August, 1989) } \\
\end{array}$} & \multicolumn{3}{|c|}{$\begin{array}{c}\text { Increasing } \\
(\text { January, 1985) }\end{array}$} & \multicolumn{3}{|c|}{$\begin{array}{c}\text { Decreasing } \\
(\text { May, 1981) }\end{array}$} & \multicolumn{3}{|c|}{$\begin{array}{c}\text { Humped } \\
\text { (October, 1986) }\end{array}$} \\
\hline & 1 year & 5 year & 10 year & 1 year & 5 year & 10 year & 1 year & 5 year & 10 year & 1 year & 5 year & 10 year \\
\hline \multicolumn{13}{|l|}{ Macaulay } \\
\hline Bond maturities & $12,13^{*}$ & 120,302 & 148,264 & 12,44 & 111,255 & 156,309 & 19,111 & 80,263 & 176,342 & 12,15 & 100,132 & 140,268 \\
\hline \multirow[t]{2}{*}{ Return } & $8.1523 \%$ & $8.2944 \%$ & $8.1269 \%$ & $8.8664 \%$ & $10.8971 \%$ & $11.0437 \%$ & $14.7929 \%$ & $13.3716 \%$ & $12.9075 \%$ & $5.6248 \%$ & $6.9571 \%$ & $7.5556 \%$ \\
\hline & $(3.24 \mathrm{E}-06)$ & (1.54E-09) & $(1.33 \mathrm{E}-10)$ & $(2.78 \mathrm{E}-12)$ & $(6.99 \mathrm{E}-10)$ & $(1.10 \mathrm{E}-10)$ & (2.90E-09) & $(4.20 \mathrm{E}-10)$ & $(6.85 \mathrm{E}-11)$ & $(2.53 \mathrm{E}-11)$ & (1.32E-09) & $(1.89 \mathrm{E}-10)$ \\
\hline $\mathrm{AD}$ & $2.36 \mathrm{E}-07$ & $1.40 \mathrm{E}-03$ & $1.96 \mathrm{E}-04$ & $2.43 \mathrm{E}-06$ & $1.58 \mathrm{E}-03$ & $5.53 \mathrm{E}-04$ & $9.69 \mathrm{E}-05$ & $2.21 \mathrm{E}-04$ & $3.93 \mathrm{E}-05$ & $5.31 \mathrm{E}-07$ & $5.77 \mathrm{E}-04$ & $6.61 \mathrm{E}-04$ \\
\hline $\mathrm{RD}$ & $2.89 \mathrm{E}-06$ & $1.72 \mathrm{E}-02$ & $2.41 \mathrm{E}-03$ & $2.74 \mathrm{E}-05$ & $1.47 \mathrm{E}-02$ & $4.98 \mathrm{E}-03$ & $6.55 \mathrm{E}-04$ & $1.66 \mathrm{E}-03$ & $3.04 \mathrm{E}-04$ & $9.44 \mathrm{E}-06$ & $8.36 \mathrm{E}-03$ & $8.68 \mathrm{E}-03$ \\
\hline \multicolumn{13}{|l|}{ Fisher-Weil } \\
\hline Bond maturities & $12,13 *$ & 101,171 & 144,225 & 12,53 & 98,269 & 123,285 & 12,79 & 85,242 & 139,332 & 12,15 & 71,86 & 126,276 \\
\hline \multirow[t]{2}{*}{ Return } & $8.1523 \%$ & $8.2033 \%$ & $8.1222 \%$ & $8.8664 \%$ & $10.8608 \%$ & $10.9699 \%$ & $14.7831 \%$ & $13.3808 \%$ & $12.8570 \%$ & $5.6248 \%$ & $6.8990 \%$ & $7.6099 \%$ \\
\hline & $(3.23 \mathrm{E}-06)$ & $(2.32 \mathrm{E}-09)$ & $(2.82 \mathrm{E}-11)$ & $(5.05 \mathrm{E}-12)$ & $(7.48 \mathrm{E}-10)$ & $(2.08 \mathrm{E}-10)$ & $(5.49 \mathrm{E}-12)$ & $(1.04 \mathrm{E}-10)$ & $(1.30 \mathrm{E}-10)$ & $(2.53 \mathrm{E}-11)$ & $(5.11 \mathrm{E}-10)$ & $(1.77 \mathrm{E}-08)$ \\
\hline $\mathrm{AD}$ & $2.32 \mathrm{E}-07$ & $4.87 \mathrm{E}-04$ & $2.43 \mathrm{E}-04$ & $2.92 \mathrm{E}-06$ & $1.22 \mathrm{E}-03$ & $1.29 \mathrm{E}-03$ & $1.65 \mathrm{E}-06$ & $3.13 \mathrm{E}-04$ & $4.66 \mathrm{E}-04$ & $5.30 \mathrm{E}-07$ & $4.05 \mathrm{E}-06$ & $1.19 \mathrm{E}-04$ \\
\hline $\mathrm{RD}$ & $2.85 \mathrm{E}-06$ & $5.97 \mathrm{E}-03$ & $2.98 \mathrm{E}-03$ & $3.30 \mathrm{E}-05$ & $1.14 \mathrm{E}-02$ & $1.16 \mathrm{E}-02$ & $1.12 \mathrm{E}-05$ & $2.35 \mathrm{E}-03$ & $3.61 \mathrm{E}-03$ & $9.42 \mathrm{E}-06$ & $5.87 \mathrm{E}-05$ & $1.56 \mathrm{E}-03$ \\
\hline
\end{tabular}

* $\quad$ Bullet portfolio 
Table 23 - Continued

\begin{tabular}{|c|c|c|c|c|c|c|c|c|c|c|c|c|}
\hline \multicolumn{13}{|c|}{$\begin{array}{c}\text { Panel B } \\
\text { Duration and Convexity Matched Minimum M-Square Portfolios }\end{array}$} \\
\hline & \multicolumn{3}{|c|}{$\begin{array}{c}\text { Flat } \\
\text { (August, 1989) }\end{array}$} & \multicolumn{3}{|c|}{$\begin{array}{c}\text { Increasing } \\
\text { (January, 1985) }\end{array}$} & \multicolumn{3}{|c|}{$\begin{array}{l}\text { Decreasing } \\
\text { (May, 1981) }\end{array}$} & \multicolumn{3}{|c|}{$\begin{array}{c}\text { Humped } \\
\text { (October, 1986) }\end{array}$} \\
\hline & 1 year & 5 year & 10 year & 1 year & 5 year & 10 year & 1 year & 5 year & 10 year & 1 year & 5 year & 10 year \\
\hline \multicolumn{13}{|l|}{ Macaulay } \\
\hline Bond maturities & $12,13,14^{*}$ & $60,63,79$ & $120,140,182$ & $12,19,201$ & $60,64,66$ & $120,139,288$ & $12,20,140$ & $60,64,66$ & $120,131,321$ & $12,13,14^{*}$ & $60,61,66$ & $120,133,225$ \\
\hline \multirow[t]{2}{*}{ Return } & $8.1523 \%$ & $8.1543 \%$ & $8.1469 \%$ & $8.8667 \%$ & $10.7386 \%$ & $11.1129 \%$ & $14.7832 \%$ & $13.3494 \%$ & $12.8821 \%$ & $5.6248 \%$ & $6.8993 \%$ & $7.6374 \%$ \\
\hline & $(1.25 \mathrm{E}-05)$ & $(2.19 \mathrm{E}-10)$ & $(2.39 \mathrm{E}-11)$ & (3.36E-14) & $(6.40 \mathrm{E}-11)$ & (7.04E-08) & $(2.20 \mathrm{E}-15)$ & (1.98E-13) & $(1.75 \mathrm{E}-07)$ & $(1.81 \mathrm{E}-05)$ & $(7.66 \mathrm{E}-11)$ & $(2.80 \mathrm{E}-08)$ \\
\hline $\mathrm{AD}$ & 2.35E-09 & $2.43 \mathrm{E}-06$ & $3.44 \mathrm{E}-06$ & $1.28 \mathrm{E}-08$ & $1.88 \mathrm{E}-06$ & $1.39 \mathrm{E}-04$ & $1.53 \mathrm{E}-08$ & $2.77 \mathrm{E}-07$ & $2.15 \mathrm{E}-04$ & $6.94 \mathrm{E}-09$ & $1.32 \mathrm{E}-06$ & $1.56 \mathrm{E}-04$ \\
\hline $\mathrm{RD}$ & $2.88 \mathrm{E}-08$ & $2.98 \mathrm{E}-05$ & $4.23 \mathrm{E}-05$ & $1.44 \mathrm{E}-07$ & $1.75 \mathrm{E}-05$ & $1.25 \mathrm{E}-03$ & $1.03 \mathrm{E}-07$ & $2.07 \mathrm{E}-06$ & $1.67 \mathrm{E}-03$ & $1.23 \mathrm{E}-07$ & $1.91 \mathrm{E}-05$ & $2.05 \mathrm{E}-03$ \\
\hline \multicolumn{13}{|l|}{ Fisher-Weil } \\
\hline Bond maturities & $12,13,14^{*}$ & $60,61,66$ & $12,134,175$ & $12,16,136$ & $60,64,66$ & $120,123,236$ & $12,18,50$ & $60,64,66$ & $120,139,288$ & $12,13,14^{*}$ & $60,64,84$ & $120,134,191$ \\
\hline \multirow[t]{2}{*}{ Return } & $8.1523 \%$ & $8.1545 \%$ & $8.1511 \%$ & $8.8667 \%$ & $10.7387 \%$ & $11.0949 \%$ & $14.7832 \%$ & $13.3494 \%$ & $12.8974 \%$ & $5.6248 \%$ & $6.8949 \%$ & $7.6462 \%$ \\
\hline & $(1.25 \mathrm{E}-05)$ & $(1.65 \mathrm{E}-08)$ & $(1.59 \mathrm{E}-06)$ & $(4.69 \mathrm{E}-12)$ & (1.86E-110 & (7.65E-080 & (7.30E-150 & $(5.73 \mathrm{E}-12)$ & $(9.25 \mathrm{E}-08)$ & $(1.81 \mathrm{E}-05)$ & (6.51E-06) & (1.75E-07) \\
\hline $\mathrm{AD}$ & 2.37E-09 & $5.21 \mathrm{E}-07$ & $4.57 \mathrm{E}-05$ & 6.37E-09 & $1.34 \mathrm{E}-06$ & $4.12 \mathrm{E}-05$ & 2.64E-09 & $4.65 \mathrm{E}-07$ & $6.23 \mathrm{E}-05$ & $6.96 \mathrm{E}-09$ & 4.49E-05 & $2.45 \mathrm{E}-04$ \\
\hline $\mathrm{RD}$ & $2.90 \mathrm{E}-08$ & $6.39 \mathrm{E}-06$ & $5.62 \mathrm{E}-04$ & 7.18E-08 & $1.25 \mathrm{E}-05$ & $3.71 \mathrm{E}-04$ & $1.78 \mathrm{E}-08$ & $3.48 \mathrm{E}-06$ & 4.82E-04 & $1.24 \mathrm{E}-07$ & $6.51 \mathrm{E}-04$ & $3.21 \mathrm{E}-03$ \\
\hline
\end{tabular}

* Bullet portfolio 
parallel and therefore traditional duration measures provide sufficient immunization. On the other hand, minimum M-square portfolios are not the ones that have the closest returns to the target. It is so only in one case where the initial yield curve is humped, the holding period is five years, and the risk measure is the Fisher-Weil duration.

Under the passive portfolio management criterion, the closest returns to the target are observed for short holding periods. All portfolios have returns within one basis point of the target for short holding periods irrespective of the duration measure. As the holding period increases, the deviation of the minimum M-square portfolio returns from the target yield increases, though most of the portfolio returns are still within 16 basis points of the target. This result is expected, since the variance of time-to-payments around duration increases with the holding period.

Bullet portfolios are minimum M-square portfolios only under a flat initial yield curve and for a one-year holding period. Bierwag, Fooladi and Roberts (1993) show that minimum M-square portfolios are bullet portfolios if and only if minimum M-square portfolios are strictly convex functions of duration. Since this condition cannot be satisfied in each case, we should not expect to observe bullet portfolios as minimum M-square portfolios.

Table 23, Panel B shows the results of the duration and convexity matched minimum M-square portfolios. All portfolios are successfully immunized according to the active portfolio strategies. Yet none has the closest return to the target. The deviation of portfolio returns from the target yield increases with the holding period. All portfolio returns are within one basis points of the target for short to medium holding periods. For long holding periods, all portfolios have returns within three basis points of the target. In this respect, matching with both duration and convexity has additional benefits for long holding periods when the minimum M-square strategy is used. The portfolio returns are closer to the target by 3 basis points with the duration and convexity matching strategy, whereas they are within 15 basis points of the target with only the duration matching strategy. In this respect, the improvement by duration and convexity matching is dramatic. Bullet portfolios are minimum M-square portfolios for one-year holding periods under flat and humped initial yield curves. 
These results suggest that under the constant volatility HJM framework, with the minimum M-square strategy, the duration matching strategy is sufficient for short holding periods under both active and passive portfolio management. The duration matching strategy is also sufficient for medium to long holding periods under active portfolio management but not under passive portfolio management. For risk managers who pursue passive portfolio management, there is significant benefit of using the duration and convexity matching strategy for minimum M-square portfolios for medium to long holding periods. Therefore, overall, the duration and convexity matching strategy improves the immunization performance of minimum M-square portfolios significantly under passive portfolio management.

\subsection{Exponential Decay Volatility One-Factor HJM Framework}

Under this framework, the benchmark risk measures are the HJM risk measures developed for exponentially decaying forward rate volatilities. These HJM risk measures should be sufficient in immunization under an exponential decay volatility HJM framework. Therefore, minimizing the M-squares of the portfolios formed by the HJM risk measures should not improve immunization performance significantly. With traditional risk measures, however, minimizing M-square can improve the immunization performance.

Table 24, Panel A, shows the results on the performances of duration matched minimum M-square portfolios. Under the active portfolio management criterion, all portfolios are successfully immunized. Under the passive portfolio management criterion, all portfolios have returns within one basis point of the target for short holding periods irrespective of the risk measures used for immunization. For medium to long holding periods, however, portfolios that are duration matched with the HJM duration measure are within seven basis points of the target, whereas portfolio that are matched with Macaulay's and Fisher-Weil duration measures are within 15 basis points of the target. These results support the evidence given in the previous subsection that the minimum M-square strategy is less effective as the holding period increases, since the time to payment variance increases by holding period. Also we see that using HJM duration measures instead of traditional ones has benefits for medium to long holding periods. 
Table 24

\section{Performance of Minimum M-Square Portfolios under Exponential Decay Volatility HJM Framework}

The performances of portfolios formed by minimizing the M-squares are summarized. Minimum M-square portfolios are formed for both duration matched and duration and convexity matched portfolios. Panel A gives the results of the duration matched minimum M-square portfolios. Panel B gives the results of the duration and convexity matched minimum Msquare portfolios. Three risk measures are considered. Two of these are traditional risk measures of Macaulay and Fisher-Weil. The other one is the HJM risk measure developed for the exponential decay volatility HJM framework. In this respect, HJM risk measures provide the benchmark. For each risk measure used for duration, and duration-convexity matching, bond maturities of minimum M-square portfolios are shown in the first row. The second row shows the holding period return of these portfolios. Standard deviations are given in parentheses. Next two rows show the absolute and relative deviation of the holding period returns from the target yield.

\begin{tabular}{|c|c|c|c|c|c|c|c|c|c|c|c|c|}
\hline \multicolumn{13}{|c|}{$\begin{array}{c}\text { Panel A } \\
\text { Duration Matched Minimum M-Square Portfolios }\end{array}$} \\
\hline & \multicolumn{3}{|c|}{$\begin{array}{c}\text { Flat } \\
\text { (August, 1989) }\end{array}$} & \multicolumn{3}{|c|}{$\begin{array}{c}\text { Increasing } \\
\text { (January, 1985) }\end{array}$} & \multicolumn{3}{|c|}{$\begin{array}{l}\text { Decreasing } \\
\text { (May, 1981) }\end{array}$} & \multicolumn{3}{|c|}{$\begin{array}{c}\text { Humped } \\
\text { (October, 1986) }\end{array}$} \\
\hline & 1 year & 5 year & 10 year & 1 year & 5 year & 10 year & 1 year & 5 year & 10 year & 1 year & 5 year & 10 year \\
\hline \multicolumn{13}{|l|}{ Macaulay } \\
\hline Bond maturities & $12,13^{*}$ & 120,302 & 148,264 & 12,44 & 111,255 & 156,309 & 19,111 & 80,263 & 176,342 & 12,15 & 100,132 & 140,268 \\
\hline \multirow[t]{2}{*}{ Return } & $8.1523 \%$ & $8.2594 \%$ & $8.0943 \%$ & $8.8665 \%$ & $10.9600 \%$ & $10.9600 \%$ & $14.7890 \%$ & $13.3633 \%$ & $12.8922 \%$ & $5.6248 \%$ & $6.9182 \%$ & $7.4948 \%$ \\
\hline & $(3.69 \mathrm{E}-06)$ & (2.61E-09) & $(5.05 \mathrm{E}-09)$ & $(1.44 \mathrm{E}-12)$ & (1.28E-08) & $(1.28 \mathrm{E}-08)$ & (1.54E-09) & $(2.31 \mathrm{E}-10)$ & $(2.41 \mathrm{E}-10)$ & $(1.21 \mathrm{E}-11)$ & $(3.84 \mathrm{E}-10)$ & $(1.68 \mathrm{E}-08)$ \\
\hline $\mathrm{AD}$ & $1.80 \mathrm{E}-07$ & $1.05 \mathrm{E}-03$ & $5.22 \mathrm{E}-04$ & $1.26 \mathrm{E}-06$ & $1.39 \mathrm{E}-03$ & $1.39 \mathrm{E}-03$ & $5.76 \mathrm{E}-05$ & $1.38 \mathrm{E}-04$ & $1.14 \mathrm{E}-04$ & $3.43 \mathrm{E}-07$ & $1.88 \mathrm{E}-04$ & $1.27 \mathrm{E}-03$ \\
\hline $\mathrm{RD}$ & $2.20 \mathrm{E}-06$ & $1.27 \mathrm{E}-02$ & $6.45 \mathrm{E}-03$ & $1.42 \mathrm{E}-05$ & $1.27 \mathrm{E}-02$ & $1.27 \mathrm{E}-02$ & $3.90 \mathrm{E}-04$ & $1.03 \mathrm{E}-03$ & 8.87E-04 & $6.10 \mathrm{E}-06$ & $2.72 \mathrm{E}-03$ & $1.69 \mathrm{E}-02$ \\
\hline \multicolumn{13}{|l|}{ Fisher-Weil } \\
\hline Bond maturities & $12,13 *$ & 101,171 & 144,225 & 12,53 & 98,269 & 123,285 & 12,79 & 85,242 & 139,332 & 12,15 & 71,86 & 126,276 \\
\hline \multirow[t]{2}{*}{ Return } & $8.1523 \%$ & $8.2594 \%$ & $8.0943 \%$ & $8.8665 \%$ & $10.9600 \%$ & $10.9600 \%$ & $14.7890 \%$ & $13.3633 \%$ & $12.8922 \%$ & $5.6248 \%$ & $6.9182 \%$ & $7.4948 \%$ \\
\hline & $(3.69 \mathrm{E}-06)$ & (2.61E-090 & $(5.05 \mathrm{E}-09)$ & $(1.44 \mathrm{E}-12)$ & $(1.28 \mathrm{E}-08)$ & $(1.28 \mathrm{E}-08)$ & 91.54E-09) & $(2.31 \mathrm{E}-10)$ & $(2.41 \mathrm{E}-10)$ & $(1.21 \mathrm{E}-11)$ & $(3.84 \mathrm{E}-10)$ & $(1.68 \mathrm{E}-08)$ \\
\hline $\mathrm{AD}$ & $1.80 \mathrm{E}-07$ & $1.05 \mathrm{E}-03$ & $5.22 \mathrm{E}-04$ & $1.26 \mathrm{E}-06$ & $1.39 \mathrm{E}-03$ & $1.39 \mathrm{E}-03$ & $5.76 \mathrm{E}-05$ & $1.38 \mathrm{E}-04$ & $1.14 \mathrm{E}-04$ & $3.43 \mathrm{E}-07$ & $1.88 \mathrm{E}-04$ & $1.27 \mathrm{E}-03$ \\
\hline $\mathrm{RD}$ & $2.20 \mathrm{E}-06$ & $1.27 \mathrm{E}-02$ & $6.45 \mathrm{E}-03$ & $1.42 \mathrm{E}-05$ & $1.27 \mathrm{E}-02$ & $1.27 \mathrm{E}-02$ & $3.90 \mathrm{E}-04$ & $1.03 \mathrm{E}-03$ & $8.87 \mathrm{E}-04$ & $6.10 \mathrm{E}-06$ & $2.72 \mathrm{E}-03$ & $1.69 \mathrm{E}-02$ \\
\hline \multicolumn{13}{|l|}{ HJM } \\
\hline Bond maturities & 27,99 & 137,216 & 188,306 & 12,47 & 77,276 & 193,269 & 12,112 & 78,325 & 126,204 & 16,29 & 81,163 & 124,166 \\
\hline \multirow[t]{2}{*}{ Return } & $8.1615 \%$ & $8.2195 \%$ & $8.1735 \%$ & $8.8666 \%$ & $10.7562 \%$ & $11.1016 \%$ & $14.7832 \%$ & $13.3493 \%$ & $12.9006 \%$ & $5.6248 \%$ & $6.9002 \%$ & $7.6234 \%$ \\
\hline & $(3.75 \mathrm{E}-09)$ & $(1.83 \mathrm{E}-10)$ & $(1.69 \mathrm{E}-11)$ & $(3.11 \mathrm{E}-12)$ & $(1.50 \mathrm{E}-10)$ & $(1.03 \mathrm{E}-10)$ & $(1.60 \mathrm{E}-12)$ & $(2.36 \mathrm{E}-10)$ & $(1.94 \mathrm{E}-09)$ & $(7.35 \mathrm{E}-12)$ & $(1.63 \mathrm{E}-10)$ & $(8.54 \mathrm{E}-11)$ \\
\hline $\mathrm{AD}$ & $9.15 \mathrm{E}-05$ & $6.49 \mathrm{E}-04$ & $2.70 \mathrm{E}-04$ & $7.30 \mathrm{E}-07$ & $1.74 \mathrm{E}-04$ & $2.63 \mathrm{E}-05$ & $4.41 \mathrm{E}-08$ & $1.44 \mathrm{E}-06$ & $3.00 \mathrm{E}-05$ & $1.05 \mathrm{E}-08$ & $7.98 \mathrm{E}-06$ & $1.62 \mathrm{E}-05$ \\
\hline $\mathrm{RD}$ & $1.12 \mathrm{E}-03$ & $7.96 \mathrm{E}-03$ & $3.31 \mathrm{E}-03$ & $8.24 \mathrm{E}-06$ & $1.62 \mathrm{E}-03$ & $2.37 \mathrm{E}-04$ & $2.98 \mathrm{E}-07$ & $1.08 \mathrm{E}-05$ & 2.33E-04 & $1.87 \mathrm{E}-07$ & $1.16 \mathrm{E}-04$ & $2.13 \mathrm{E}-04$ \\
\hline
\end{tabular}


Table 24 - Continued

\begin{tabular}{|c|c|c|c|c|c|c|c|c|c|c|c|c|}
\hline \multicolumn{13}{|c|}{$\begin{array}{c}\text { Panel B } \\
\text { Duration and Convexity Matched Minimum M-Square Portfolios }\end{array}$} \\
\hline & \multicolumn{3}{|c|}{$\begin{array}{c}\text { Flat } \\
\text { (August, 1989) }\end{array}$} & \multicolumn{3}{|c|}{$\begin{array}{c}\text { Increasing } \\
\text { (January, 1985) }\end{array}$} & \multicolumn{3}{|c|}{$\begin{array}{c}\text { Decreasing } \\
(\text { May, 1981) }\end{array}$} & \multicolumn{3}{|c|}{$\begin{array}{c}\text { Humped } \\
\text { (October, 1986) }\end{array}$} \\
\hline & 1 year & 5 year & 10 year & 1 year & 5 year & 10 year & 1 year & 5 year & 10 year & 1 year & 5 year & 10 year \\
\hline \multicolumn{13}{|l|}{ Macaulay } \\
\hline Bond maturities & $12,13,14^{*}$ & $60,63,79$ & $120,140,182$ & $12,19,201$ & $60,64,66$ & $120,139,288$ & $12,20,140$ & $60,64,66$ & $120,131,321$ & $12,13,14^{*}$ & $60,61,66$ & $120,133,225$ \\
\hline \multirow[t]{2}{*}{ Return } & $8.1523 \%$ & $8.1528 \%$ & $8.1109 \%$ & $8.8667 \%$ & $10.7359 \%$ & $11.0781 \%$ & $14.7832 \%$ & $13.3487 \%$ & $12.8973 \%$ & $5.6248 \%$ & $6.8967 \%$ & $7.5983 \%$ \\
\hline & $(8.06 \mathrm{E}-06)$ & (4.41E-09) & $(1.41 \mathrm{E}-07)$ & $(1.58 \mathrm{E}-11)$ & $(1.38 \mathrm{E}-08)$ & $(1.17 \mathrm{E}-07)$ & $(2.14 \mathrm{E}-12)$ & $(1.22 \mathrm{E}-09)$ & $(2.33 \mathrm{E}-07)$ & $(1.07 \mathrm{E}-05)$ & $(1.41 \mathrm{E}-08)$ & $(2.45 \mathrm{E}-07)$ \\
\hline $\mathrm{AD}$ & $9.19 \mathrm{E}-08$ & $1.73 \mathrm{E}-05$ & $3.56 \mathrm{E}-04$ & $5.14 \mathrm{E}-08$ & $2.86 \mathrm{E}-05$ & 2.09E-04 & $3.17 \mathrm{E}-08$ & 7.64E-06 & $6.32 \mathrm{E}-05$ & $1.73 \mathrm{E}-07$ & $2.71 \mathrm{E}-05$ & $2.35 \mathrm{E}-04$ \\
\hline $\mathrm{RD}$ & $1.13 \mathrm{E}-06$ & $2.13 \mathrm{E}-04$ & 4.37E-03 & $5.79 \mathrm{E}-07$ & $2.67 \mathrm{E}-04$ & $1.88 \mathrm{E}-03$ & $2.15 \mathrm{E}-07$ & $5.72 \mathrm{E}-05$ & $4.90 \mathrm{E}-04$ & $3.07 \mathrm{E}-06$ & $3.93 \mathrm{E}-04$ & $3.08 \mathrm{E}-03$ \\
\hline \multicolumn{13}{|l|}{ Fisher-Weil } \\
\hline Bond maturities & $12,13,14^{*}$ & $60,61,66$ & $12,134,175$ & $12,16,136$ & $60,64,66$ & $120,123,236$ & $12,18,50$ & $60,64,66$ & $120,139,288$ & $12,13,14^{*}$ & $60,64,84$ & $120,134,191$ \\
\hline \multirow[t]{2}{*}{ Return } & $8.1523 \%$ & $8.1528 \%$ & $8.0509 \%$ & $8.8667 \%$ & $10.7360 \%$ & $11.0420 \%$ & $14.7832 \%$ & $13.3487 \%$ & $12.8415 \%$ & $5.6248 \%$ & $6.9049 \%$ & $7.5783 \%$ \\
\hline & $(8.05 \mathrm{E}-06)$ & $(5.76 \mathrm{E}-09)$ & $(1.34 \mathrm{E}-05)$ & $(1.21 \mathrm{E}-11)$ & $(1.32 \mathrm{E}-08)$ & (1.48E-07) & $(3.42 \mathrm{E}-13)$ & $(1.34 \mathrm{E}-09)$ & (2.64E-07) & (1.07E-05) & $(3.68 \mathrm{E}-06)$ & $(1.05 \mathrm{E}-07)$ \\
\hline $\mathrm{AD}$ & $9.20 \mathrm{E}-08$ & $1.37 \mathrm{E}-05$ & $9.56 \mathrm{E}-04$ & $6.85 \mathrm{E}-08$ & $2.84 \mathrm{E}-05$ & $5.70 \mathrm{E}-04$ & 4.72E-08 & $7.76 \mathrm{E}-06$ & $6.21 \mathrm{E}-04$ & $1.73 \mathrm{E}-07$ & $5.52 \mathrm{E}-05$ & $4.35 \mathrm{E}-04$ \\
\hline $\mathrm{RD}$ & $1.13 \mathrm{E}-06$ & $1.68 \mathrm{E}-04$ & $1.17 \mathrm{E}-02$ & 7.72E-07 & 2.64E-04 & $5.14 \mathrm{E}-03$ & $3.19 \mathrm{E}-07$ & $5.81 \mathrm{E}-05$ & $4.81 \mathrm{E}-03$ & $3.07 \mathrm{E}-06$ & 8.01E-04 & $5.70 \mathrm{E}-03$ \\
\hline \multicolumn{13}{|l|}{ HJM } \\
\hline Bond maturities & $12,13,14^{*}$ & $60,61,66$ & $120,134,175$ & $12,22,226$ & $60,66,76$ & $120,122,162$ & $12,27,80$ & $60,66,76$ & $120,122,162$ & $12,13,14^{*}$ & $60,67,72$ & $120,131,158$ \\
\hline \multirow[t]{2}{*}{ Return } & $8.1523 \%$ & $8.1545 \%$ & $8.1463 \%$ & $8.8667 \%$ & $10.7388 \%$ & $11.0977 \%$ & $14.7832 \%$ & $13.3495 \%$ & $12.9029 \%$ & $5.6248 \%$ & $6.8975 \%$ & $7.6218 \%$ \\
\hline & $(6.89 \mathrm{E}-06)$ & $(1.22 \mathrm{E}-11)$ & $(2.83 \mathrm{E}-08)$ & $(9.00 \mathrm{E}-16)$ & $(2.00 \mathrm{E}-13)$ & $(8.90 \mathrm{E}-10)$ & $(1.00 \mathrm{E}-16)$ & $(4.65 \mathrm{E}-13)$ & $(4.45 \mathrm{E}-10)$ & $(9.03 \mathrm{E}-06)$ & $(2.08 \mathrm{E}-09)$ & $(1.45 \mathrm{E}-10)$ \\
\hline $\mathrm{AD}$ & 7.23E-10 & $1.97 \mathrm{E}-07$ & $2.63 \mathrm{E}-06$ & $2.58 \mathrm{E}-09$ & $1.02 \mathrm{E}-08$ & $1.23 \mathrm{E}-05$ & $6.01 \mathrm{E}-09$ & $1.06 \mathrm{E}-07$ & $7.10 \mathrm{E}-06$ & $1.76 \mathrm{E}-09$ & $1.91 \mathrm{E}-05$ & $1.01 \mathrm{E}-06$ \\
\hline $\mathrm{RD}$ & 8.87E-09 & $2.42 \mathrm{E}-06$ & $3.23 \mathrm{E}-05$ & $2.91 \mathrm{E}-08$ & $9.51 \mathrm{E}-08$ & $1.11 \mathrm{E}-04$ & 4.07E-08 & $7.94 \mathrm{E}-07$ & $5.50 \mathrm{E}-05$ & $3.14 \mathrm{E}-08$ & $2.77 \mathrm{E}-04$ & $1.32 \mathrm{E}-05$ \\
\hline
\end{tabular}

* Bullet portfolio 
Only two of the portfolios have the closest holding period returns to the target. Both of these are formed using HJM risk measures. One of them is formed for a decreasing initial yield curve and five-year holding period. The other is formed for a humped initial yield curve and one-year holding period. Only two bullet portfolios are minimum M-square portfolios. These bullet portfolios are formed by traditional risk measures under a flat initial yield curve and for a one-year holding period.

As expected, minimizing the M-squares of the portfolios formed by the HJM duration measures leads to closer returns to the target than minimizing the M-squares of the portfolios formed by traditional risk measures, especially for medium to long holding periods where the time to payment variance is relatively high. This result shows that minimizing M-square has benefits but still the selection of the correct interest rate risk measure is an important step in immunization. Also, the results suggest that bullet portfolios are preferable to minimum Msquare portfolios for short holding periods when the duration matching strategy is used since bullet portfolios have the closest returns to the target for short holding periods. For medium to long holding periods, minimum M-square, bullet, and barbell portfolios do not have significantly different performances.

The results on the performances of duration and convexity matched minimum Msquare portfolios are given in Table 24, Panel B. Under the active portfolio management criterion, all minimum M-square portfolios are successfully immunized. Additionally, all portfolios have returns within one basis point of the target for short and medium holding periods irrespective of the risk measure used for immunization. For long holding periods, the HJM duration and convexity measures still lead to portfolio returns within one basis point of the target. Portfolio returns for long holding periods are within four basis points of the target with Macaulay's risk measures and within ten basis points with the Fisher-Weil risk measures. We again observe that for medium to long holding periods, matching with convexity in addition to duration improves the immunization performance of minimum M-square portfolios under the passive portfolio management criterion. We also observe that the performance of M-square decreases with holding period since the time to payment variance increases with time to maturity. Also, choosing the correct risk measure is still important for 
long holding periods. All these results support the evidence presented under the constant volatility HJM framework.

There are four portfolios that have the closest returns to the target. These are formed by traditional risk measures under increasing and decreasing initial yield curves, and for a one-year holding period. As expected, when we use the duration and convexity matching strategy, the deviation of returns from the target is the lowest for short holding periods and the highest for long holding periods. Long holding periods correspond to high volatility segments of the yield curve, and vice versa. Therefore, we expect to observe higher variation for long holding periods. Bullet portfolios are minimum M-square portfolios for one-year holding periods under flat and humped initial yield curves.

When we adjust for convexity and use the duration and convexity matching strategy, we again observe that minimizing the M-square of duration and convexity matched portfolios does not lead to significant improvement in the immunization performance according to the active portfolio management criterion. Minimum M-square portfolios formed with the HJM risk measures have closer returns to the target than those formed with traditional risk measures. In general, the duration and convexity matched portfolios have closer returns to the target than the duration-matched portfolios. Since convexity captures the nonlinear relation between the bond price and the yield change, the duration and convexity matching strategy is expected to have closer returns to the target compared to the duration matching strategy. For short holding periods, bullet, barbell and minimum M-square portfolios produce very close returns to the target. Therefore, risk managers who want to have close returns to a certain target yield, can use any of these portfolio strategies for short holding periods. Overall, using

minimum M-square portfolios with the duration and convexity matching strategy has considerable benefits for long holding periods, especially under passive portfolio management criterion.

\subsection{Constant Decay Volatility One-Factor HJM Framework}

Under the constant decay volatility HJM framework, simulated forward rates have volatilities that decay at a constant rate with time to maturity. Risk measures derived for this 
specific HJM model are the benchmarks under this framework. We do not expect to observe a significant improvement in immunization performance when we minimize M-squares of the portfolios formed by HJM risk measures. We do expect to observe improvement in the immunization performance of portfolios formed by traditional risk measures. Under this specific HJM framework, yield curve shifts are not necessarily parallel. Therefore, minimizing the M-squares of portfolios formed by traditional risk measures should provide better immunization.

Table 25, Panel A shows the results on the performances of the duration-matched minimum M-square portfolios. Under the active portfolio management criterion, all minimum M-square portfolios are successful. Under the criteria that correspond to passive portfolio management, all portfolios have returns within one basis of the target for short holding periods, irrespective of the duration measures used for immunization. For the medium holding period, portfolios that are matched with the Fisher-Weil or HJM duration measures have returns within five basis of the target, and those that are matched with Macaulay's duration have returns within nine basis points of the target. For long holding periods, portfolio returns are within ten basis points of the target with HJM risk measures and within 23 and 28 basis points with Macaulay's and Fisher-Weil duration measures, respectively. In these respects, the minimum M-square portfolios are more sensitive to holding periods than the volatility levels. Under this HJM framework, the highest volatility levels are observed for short holding periods. The minimum M-square portfolio returns, however, are within one basis point for these short holding periods. On the other hand, minimum M-square portfolio returns are within 10 to 28 basis points of the target for long holding periods when the volatility is relatively low. Therefore, these results suggest that the increase in the time to payment variance caused by the increase in the holding period has a more significant effect on the immunization performance of minimum M-square portfolios than does the volatility level.

Minimizing M-square leads to the closest returns to the target with all risk measures for a flat initial yield curve and ten-year holding period. In all other cases, minimum M-square portfolios are not the ones with the closest returns to the target. Bullet portfolios are the 
Table 25

\section{Performance of Minimum M-Square Portfolios under Constant Decay Volatility HJM Framework}

The performances of portfolios formed by minimizing the M-squares are summarized. Minimum M-square portfolios are formed for both duration matched and duration and convexity matched portfolios. Panel A gives the results of the duration matched minimum M-square portfolios. Panel B gives the results of the duration and convexity matched minimum Msquare portfolios. Three risk measures are considered. Two of these are traditional risk measures of Macaulay and Fisher-Weil. The other one is the HJM risk measure developed for the constant decay volatility HJM framework. In this respect, HJM risk measures provide the benchmark. For each risk measure used for duration, and duration-convexity matching, bond maturities of minimum M-square portfolios are shown in the first row. The second row shows the holding period return of these portfolios. Standard deviations are given in parentheses. The next two rows show the absolute and relative deviation of the holding period returns from the target yield.

\begin{tabular}{|c|c|c|c|c|c|c|c|c|c|c|c|c|}
\hline \multicolumn{13}{|c|}{$\begin{array}{c}\text { Panel A } \\
\text { Duration Matched Minimum M-Square Portfolios }\end{array}$} \\
\hline & \multicolumn{3}{|c|}{$\begin{array}{c}\text { Flat } \\
\text { (August, 1989) }\end{array}$} & \multicolumn{3}{|c|}{$\begin{array}{c}\text { Increasing } \\
\text { (January, 1985) }\end{array}$} & \multicolumn{3}{|c|}{$\begin{array}{l}\text { Decreasing } \\
\text { (May, 1981) }\end{array}$} & \multicolumn{3}{|c|}{$\begin{array}{c}\text { Humped } \\
\text { (October, 1986) }\end{array}$} \\
\hline & 1 year & 5 year & 10 year & 1 year & 5 year & 10 year & 1 year & 5 year & 10 year & 1 year & 5 year & 10 year \\
\hline \multicolumn{13}{|l|}{ Macaulay } \\
\hline Bond maturities & $12,13^{*}$ & 120,302 & 148,264 & 12,44 & 111,255 & 156,309 & 19,111 & 80,263 & 176,342 & 12,15 & 100,132 & 140,268 \\
\hline Return & $8.1596 \%$ & $8.1423 \%$ & $8.1423 \%$ & $8.8810 \%$ & $10.7510 \%$ & $11.2947 \%$ & $14.7877 \%$ & $13.3702 \%$ & $12.9484 \%$ & $5.6378 \%$ & $6.9811 \%$ & $7.8438 \%$ \\
\hline & (5.52E-04) & $(2.5 \mathrm{E}-07)$ & $(4.17 \mathrm{E}-09)$ & (1.91E-08) & (1.98E-07) & $(4.45 \mathrm{E}-07)$ & $(3.07 \mathrm{E}-07)$ & $(1.26 \mathrm{E}-08)$ & $(4.39 \mathrm{E}-08)$ & $(9.06 \mathrm{E}-08)$ & (5.87E-09) & $(6.91 \mathrm{E}-07)$ \\
\hline $\mathrm{AD}$ & $7.27 \mathrm{E}-05$ & $1.23 \mathrm{E}-04$ & 4.24E-05 & $1.43 \mathrm{E}-04$ & $1.23 \mathrm{E}-04$ & $1.96 \mathrm{E}-03$ & $4.43 \mathrm{E}-05$ & $2.07 \mathrm{E}-04$ & $4.48 \mathrm{E}-04$ & $1.30 \mathrm{E}-04$ & $8.17 \mathrm{E}-04$ & $2.22 \mathrm{E}-03$ \\
\hline $\mathrm{RD}$ & 8.92E-04 & $1.50 \mathrm{E}-03$ & $5.21 \mathrm{E}-04$ & $1.61 \mathrm{E}-03$ & $1.14 \mathrm{E}-03$ & $1.76 \mathrm{E}-02$ & $3.00 \mathrm{E}-04$ & $1.55 \mathrm{E}-03$ & $3.47 \mathrm{E}-03$ & $2.30 \mathrm{E}-03$ & $1.18 \mathrm{E}-02$ & $2.91 \mathrm{E}-02$ \\
\hline \multicolumn{13}{|l|}{ Fisher-Weil } \\
\hline Bond maturities & $12,13 *$ & 101,171 & 144,225 & 12,53 & 98,269 & 123,285 & 12,79 & 85,242 & 139,332 & 12,15 & 71,86 & 126,276 \\
\hline Return & $8.1596 \%$ & $8.1821 \%$ & $8.1426 \%$ & $8.8809 \%$ & $10.7803 \%$ & $11.3696 \%$ & $14.7879 \%$ & $13.3723 \%$ & $12.9845 \%$ & $5.6378 \%$ & $7.0327 \%$ & $7.8719 \%$ \\
\hline & (5.52E-04) & (3.83E-08) & $(4.00 \mathrm{E}-09)$ & (1.90E-08) & $(8.76 \mathrm{E}-08)$ & $(1.55 \mathrm{E}-06)$ & (4.37E-09) & (1.18E-08) & $(2.57 \mathrm{E}-07)$ & $(9.06 \mathrm{E}-08)$ & $(4.37 \mathrm{E}-07)$ & $(1.19 \mathrm{E}-06)$ \\
\hline $\mathrm{AD}$ & $7.27 \mathrm{E}-05$ & $2.76 \mathrm{E}-04$ & $3.92 \mathrm{E}-05$ & $1.43 \mathrm{E}-04$ & $4.15 \mathrm{E}-04$ & $2.71 \mathrm{E}-03$ & $4.63 \mathrm{E}-05$ & $2.29 \mathrm{E}-04$ & 8.09E-04 & $1.30 \mathrm{E}-04$ & $1.33 \mathrm{E}-03$ & $2.50 \mathrm{E}-03$ \\
\hline $\mathrm{RD}$ & 8.92E-04 & $3.38 \mathrm{E}-03$ & $4.81 \mathrm{E}-04$ & $1.61 \mathrm{E}-03$ & $3.87 \mathrm{E}-03$ & 2.44E-02 & $3.13 \mathrm{E}-04$ & $1.71 \mathrm{E}-03$ & $6.27 \mathrm{E}-03$ & $2.30 \mathrm{E}-03$ & $1.93 \mathrm{E}-02$ & $3.28 \mathrm{E}-02$ \\
\hline \multicolumn{13}{|l|}{ HJM } \\
\hline Bond maturities & 13,23 & 125,127 & 121,135 & 13,18 & 107,117 & 134,155 & 12,16 & 73,80 & 134,167 & 12,25 & 60,345 & 133,136 \\
\hline \multirow[t]{2}{*}{ Return } & $8.1539 \%$ & $8.1070 \%$ & $8.1463 \%$ & $8.8682 \%$ & $10.7551 \%$ & $11.0042 \%$ & $14.7830 \%$ & $13.3513 \%$ & $12.9006 \%$ & $5.6238 \%$ & $6.8606 \%$ & $7.6329 \%$ \\
\hline & $(4.61 \mathrm{E}-08)$ & (1.47E-07) & $(5.56 \mathrm{E}-08)$ & (1.08E-07) & (3.11E-07) & $(4.64 \mathrm{E}-06)$ & $(6.86 \mathrm{E}-10)$ & $(3.07 \mathrm{E}-08)$ & (1.94E-09) & $(5.16 \mathrm{E}-10)$ & (1.12E-09) & $(1.05 \mathrm{E}-06)$ \\
\hline $\mathrm{AD}$ & $1.55 \mathrm{E}-05$ & 4.75E-04 & $2.28 \mathrm{E}-06$ & $1.51 \mathrm{E}-05$ & $1.63 \mathrm{E}-03$ & $9.47 \mathrm{E}-04$ & $2.41 \mathrm{E}-06$ & $1.85 \mathrm{E}-05$ & $3.00 \mathrm{E}-05$ & $1.05 \mathrm{E}-05$ & $3.88 \mathrm{E}-04$ & $1.12 \mathrm{E}-04$ \\
\hline $\mathrm{RD}$ & $1.90 \mathrm{E}-04$ & $5.83 \mathrm{E}-03$ & $2.80 \mathrm{E}-05$ & $1.70 \mathrm{E}-04$ & $1.52 \mathrm{E}-02$ & $8.61 \mathrm{E}-03$ & $1.63 \mathrm{E}-05$ & $1.38 \mathrm{E}-04$ & $2.33 \mathrm{E}-04$ & $1.87 \mathrm{E}-04$ & $5.63 \mathrm{E}-03$ & $1.46 \mathrm{E}-03$ \\
\hline
\end{tabular}

* Bullet portfolio 
Table 25 - Continued

\begin{tabular}{|c|c|c|c|c|c|c|c|c|c|c|c|c|}
\hline \multicolumn{13}{|c|}{$\begin{array}{c}\text { Panel B } \\
\text { Duration and Convexity Matched Minimum M-Square Portfolios }\end{array}$} \\
\hline & \multicolumn{3}{|c|}{$\begin{array}{c}\text { Flat } \\
\text { (August, 1989) }\end{array}$} & \multicolumn{3}{|c|}{$\begin{array}{c}\text { Increasing } \\
(\text { January, 1985) }\end{array}$} & \multicolumn{3}{|c|}{$\begin{array}{l}\text { Decreasing } \\
(\text { May, 1981) }\end{array}$} & \multicolumn{3}{|c|}{$\begin{array}{c}\text { Humped } \\
\text { (October, 1986) }\end{array}$} \\
\hline & 1 year & 5 year & 10 year & 1 year & 5 year & 10 year & 1 year & 5 year & 10 year & 1 year & 5 year & 10 year \\
\hline \multicolumn{13}{|l|}{ Macaulay } \\
\hline Bond maturities & $12,13,14^{*}$ & $60,63,79$ & $120,140,182$ & $12,19,201$ & $60,64,66$ & $120,139,288$ & $12,20,140$ & $60,64,66$ & $120,131,321$ & $12,13,14^{*}$ & $60,61,66$ & $120,133,225$ \\
\hline \multirow[t]{2}{*}{ Return } & $8.1608 \%$ & $8.1579 \%$ & $8.1633 \%$ & $8.8681 \%$ & $10.7499 \%$ & $11.1729 \%$ & $14.7837 \%$ & $13.3544 \%$ & $12.9163 \%$ & $5.6259 \%$ & $6.9136 \%$ & $7.7016 \%$ \\
\hline & $(1.02 \mathrm{E}-04)$ & $(5.21 \mathrm{E}-05)$ & (2.09E-04) & $(1.85 \mathrm{E}-06)$ & (8.17E-05) & $(1.38 \mathrm{E}-04)$ & (6.44E-07) & $(3.28 \mathrm{E}-05)$ & $(4.01 \mathrm{E}-05)$ & $(1.21 \mathrm{E}-04)$ & $(8.32 \mathrm{E}-05)$ & (1.04E-04) \\
\hline $\mathrm{AD}$ & 8.46E-05 & $3.29 \mathrm{E}-05$ & $1.68 \mathrm{E}-04$ & 1.41E-05 & $1.11 \mathrm{E}-04$ & 7.39E-04 & 4.96E-06 & 4.92E-05 & 1.27E-04 & 1.07E-05 & $1.42 \mathrm{E}-04$ & 7.99E-04 \\
\hline $\mathrm{RD}$ & $1.04 \mathrm{E}-03$ & 4.03E-04 & - 2.06E-03 & 1.59E-04 & 1.03E-03 & 6.66E-03 & 3.36E-05 & 3.69E-04 & 9.84E-04 & 1.90E-04 & $2.06 \mathrm{E}-03$ & $1.05 E-02$ \\
\hline \multicolumn{13}{|l|}{ Fisher-Weil } \\
\hline Bond maturities & $12,13,14^{*}$ & $60,61,66$ & $12,134,175$ & $12,16,136$ & $60,64,66$ & $120,123,236$ & $12,18,50$ & $60,64,66$ & $120,139,288$ & $12,13,14^{*}$ & $60,64,84$ & $120,134,191$ \\
\hline \multirow[t]{2}{*}{ Return } & $8.1608 \%$ & $8.1618 \%$ & $8.2432 \%$ & $8.8681 \%$ & $10.7500 \%$ & $11.1227 \%$ & $14.7837 \%$ & $13.3544 \%$ & $12.9379 \%$ & $5.6259 \%$ & $6.9017 \%$ & $7.6548 \%$ \\
\hline & $(1.02 \mathrm{E}-04)$ & $(1.10 \mathrm{E}-05)$ & $(2.80 \mathrm{E}-04)$ & $(6.37 \mathrm{E}-07)$ & (8.18E-05) & $(9.61 \mathrm{E}-05)$ & $(1.42 \mathrm{E}-07)$ & $(3.28 \mathrm{E}-05)$ & $(2.96 \mathrm{E}-05)$ & $(1.21 \mathrm{E}-04)$ & $(1.07 \mathrm{E}-05)$ & $(2.47 \mathrm{E}-04)$ \\
\hline $\mathrm{AD}$ & 8.46E-05 & 7.21E-05 & 9.67E-04 & 1.35E-05 & 1.12E-04 & 2.37E-04 & 4.70E-06 & $4.93 \mathrm{E}-05$ & $3.43 \mathrm{E}-04$ & 1.07E-05 & 2.31E-05 & 3.31E-04 \\
\hline $\mathrm{RD}$ & $1.04 \mathrm{E}-03$ & 8.84E-04 & 1.19E-02 & 1.52E-04 & 1.04E-03 & $2.14 \mathrm{E}-03$ & 3.18E-05 & 3.69E-04 & $2.66 \mathrm{E}-03$ & 1.90E-04 & 3.35E-04 & 4.34E-03 \\
\hline \multicolumn{13}{|l|}{ HJM } \\
\hline Bond maturities & $12,13,26$ & $60,63,71$ & $120,139,173$ & $12,25,343$ & $60,66,74$ & $120,133,134$ & $12,13,15$ & $60,66,74$ & $120,133,154$ & $12,13,26$ & $60,64,69$ & $120,138,175$ \\
\hline \multirow[t]{2}{*}{ Return } & $8.1523 \%$ & $8.1547 \%$ & $8.1462 \%$ & $8.8666 \%$ & $10.7383 \%$ & $11.0979 \%$ & $14.7833 \%$ & $13.3493 \%$ & $12.9135 \%$ & $5.6248 \%$ & $6.8998 \%$ & $7.6191 \%$ \\
\hline & $(6.51 \mathrm{E}-10)$ & $(5.18 \mathrm{E}-07)$ & $(6.28 \mathrm{E}-08)$ & $(7.00 \mathrm{E}-12)$ & $(8.11 \mathrm{E}-11)$ & $(1.46 \mathrm{E}-10)$ & $(3.35 \mathrm{E}-10)$ & $(1.35 \mathrm{E}-11)$ & $(9.40 \mathrm{E}-07)$ & $(1.15 \mathrm{E}-09)$ & $(2.79 \mathrm{E}-11)$ & $(1.14 \mathrm{E}-05)$ \\
\hline $\mathrm{AD}$ & $8.18 \mathrm{E}-08$ & $1.31 \mathrm{E}-06$ & $3.43 \mathrm{E}-06$ & 4.87E-07 & $5.32 \mathrm{E}-06$ & $1.09 \mathrm{E}-05$ & 2.67E-08 & $1.44 \mathrm{E}-06$ & $9.87 \mathrm{E}-05$ & $3.10 \mathrm{E}-07$ & 4.47E-06 & 2.60E-05 \\
\hline $\mathrm{RD}$ & $1.00 \mathrm{E}-06$ & $1.60 \mathrm{E}-05$ & $4.21 \mathrm{E}-05$ & $5.49 \mathrm{E}-06$ & $4.95 \mathrm{E}-05$ & $9.78 \mathrm{E}-05$ & $1.80 \mathrm{E}-07$ & $1.08 \mathrm{E}-05$ & $7.65 \mathrm{E}-04$ & $5.51 \mathrm{E}-06$ & $6.48 \mathrm{E}-05$ & $3.41 \mathrm{E}-04$ \\
\hline
\end{tabular}

* Bullet portfolio 
traditional risk measures are used. As expected, minimum M-square portfolios matched with HJM risk measures have closer returns to the target than the ones matched with traditional risk measures. Bullet, barbell and minimum M-square portfolios are not significantly different from each other with respect to their immunization performance.

Under the constant decay volatility HJM framework, volatilities are high for short holding periods and low for long holding periods. On the other hand, the time-to-payment variation around duration increases with holding period. In this respect, volatility and holding period have opposite effects on duration matched M-square portfolios. When we analyze the deviation of holding period returns of minimum M-square portfolios from the target yield, we observe that the deviation is low for short holding periods and increases with the holding period. This suggests that the performance of M-square is mainly affected by the length of the holding period rather than the volatility. The shorter the holding period, the closer are the minimum M-square portfolio returns to the target. Also, minimum M-square portfolios are not very sensitive to the level of the volatility.

Table 25, Panel B shows the results on the performance of duration and convexity matched minimum M-square portfolios. All portfolios are successful under the active portfolio management criterion. When traditional risk measures are used, the immunization performances of duration and convexity matched minimum M-square portfolios are similar to those of the duration matched minimum M-square portfolios for short holding periods. For medium holding periods, the returns of the portfolio matched with Macaulay's and Fisher Weil duration and convexity measures have returns within two and four basis points of the target and for long holding periods, the portfolio returns are within eight basis points with Macaulay's risk measures and within ten basis points with Fisher-Weil risk measures. With HJM risk measures, all portfolios have returns within one basis point of the target for all holding periods. In this respect, matching minimum M-square portfolios with convexity in addition to duration has significant benefits for long holding periods, especially under the passive portfolio management criterion. Under this HJM framework, volatilities are higher for short holding periods and decrease with the holding periods. These results support our previous evidence that the performances of minimum M-square portfolios are sensitive to 
holding period and not sensitive to the level of volatility. In this respect, minimizing M-square can be preferred to other portfolio formation strategies when there is high volatility in the market. When passive portfolio management is pursued, choosing the correct risk measure, however, is still important especially for long holding periods and when using duration and convexity matching strategy.

Bullet portfolios are minimum M-square portfolios for one-year holding periods under flat and humped initial yield curves when we use traditional risk measures. The performances of minimum M-square portfolios are not very different from that of bullet and barbell portfolios for short holding periods.

\subsection{Humped Volatility One-Factor HJM Framework}

When forward rates are simulated under the humped volatility HJM framework, benchmark risk measures are the ones derived specifically for this model. Minimum M-square portfolios are formed for duration matched, and duration and convexity matched portfolios using traditional risk measures and HJM risk measures. We do not expect to observe any significant improvement in the immunization performance of minimum M-square portfolios formed by HJM risk measures. We do expect to observe improved performance for minimum M-square portfolios formed by traditional risk measures.

Table 26, Panel A shows the performances of duration matched minimum M-square portfolios. All portfolios are successful under the active portfolio management criterion. All portfolios that are matched with the HJM duration measure have returns within one basis point of the target for all periods. Portfolios matched with Macaulay's and Fisher-Weil durations have returns within one basis point of the target for short holding periods. Portfolios matched with the Fisher-Weil duration measure have returns within one basis of the target for medium periods as well. For medium to long holding periods, portfolios matched with Macaulay's duration have returns within two basis points of the target. For long holding periods portfolios matched with the Fisher-Weil duration measure have returns within two basis points of the target. In this respect, minimum M-square portfolios lead to successful immunization for all holding periods irrespective of the risk measure. 
Table 26

\section{Performance of Minimum M-Square Portfolios under Humped Volatility HJM Framework}

The performances of portfolios formed by minimizing the M-squares are summarized. Minimum M-square portfolios are formed for both duration matched and duration and convexity matched portfolios. Panel A gives the results of the duration matched minimum M-square portfolios. Panel B gives the results of the duration and convexity matched minimum Msquare portfolios. Three risk measures are considered. Two of these are traditional risk measures of Macaulay and Fisher-Weil. The other one is the HJM risk measure developed for the humped volatility HJM framework. In this respect, HJM risk measures provide the benchmark. For each risk measure used for duration, and duration-convexity matching, bond maturities of minimum M-square portfolios are shown in the first row. The second row shows the holding period return of these portfolios. Standard deviations are given in parentheses. The next two rows show the absolute and relative deviation of the holding period returns from the target yield.

\begin{tabular}{|c|c|c|c|c|c|c|c|c|c|c|c|c|}
\hline \multicolumn{13}{|c|}{$\begin{array}{c}\text { Panel A } \\
\text { Duration Matched Minimum M-Square Portfolios }\end{array}$} \\
\hline & \multicolumn{3}{|c|}{$\begin{array}{c}\text { Flat } \\
\text { (August, 1989) }\end{array}$} & \multicolumn{3}{|c|}{$\begin{array}{c}\text { Increasing } \\
\text { (January, 1985) }\end{array}$} & \multicolumn{3}{|c|}{$\begin{array}{c}\text { Decreasing } \\
\text { (May, 1981) }\end{array}$} & \multicolumn{3}{|c|}{$\begin{array}{c}\text { Humped } \\
\text { (October, 1986) }\end{array}$} \\
\hline & 1 year & 5 year & 10 year & 1 year & 5 year & 10 year & 1 year & 5 year & 10 year & 1 year & 5 year & 10 year \\
\hline \multicolumn{13}{|l|}{ Macaulay } \\
\hline Bond maturities & $12,13 *$ & 120,302 & 148,264 & 12,44 & 111,255 & 156,309 & 19,111 & 80,263 & 176,342 & 12,15 & 100,132 & 140,268 \\
\hline \multirow[t]{2}{*}{ Return } & $8.1523 \%$ & $8.1394 \%$ & $8.1423 \%$ & $8.8667 \%$ & $10.7464 \%$ & $11.0878 \%$ & $14.7837 \%$ & $13.3506 \%$ & $12.9027 \%$ & $5.6248 \%$ & $6.9010 \%$ & $7.6113 \%$ \\
\hline & $(3.81 \mathrm{E}-06)$ & $(1.45 \mathrm{E}-09)$ & $(4.17 \mathrm{E}-09)$ & $(1.45 \mathrm{E}-12)$ & $(9.54 \mathrm{E}-100$ & $(1.11 \mathrm{E}-08)$ & (1.59E-09) & $(2.37 \mathrm{E}-10)$ & $(2.22 \mathrm{E}-10)$ & $(1.22 \mathrm{E}-11)$ & $(3.87 \mathrm{E}-10)$ & $(1.55 \mathrm{E}-08)$ \\
\hline $\mathrm{AD}$ & $1.61 \mathrm{E}-08$ & $1.52 \mathrm{E}-04$ & $4.24 \mathrm{E}-05$ & $1.06 \mathrm{E}-07$ & $7.61 \mathrm{E}-05$ & $1.12 \mathrm{E}-04$ & 4.92E-06 & $1.17 \mathrm{E}-05$ & $9.05 \mathrm{E}-06$ & $2.89 \mathrm{E}-08$ & $1.59 \mathrm{E}-05$ & $1.04 \mathrm{E}-04$ \\
\hline $\mathrm{RD}$ & $1.98 \mathrm{E}-07$ & $1.86 \mathrm{E}-03$ & $5.21 \mathrm{E}-04$ & $1.20 \mathrm{E}-06$ & 7.08E-04 & $1.01 \mathrm{E}-03$ & $3.33 \mathrm{E}-05$ & $8.78 \mathrm{E}-05$ & 7.01E-05 & $5.14 \mathrm{E}-07$ & $2.31 \mathrm{E}-04$ & $1.37 \mathrm{E}-03$ \\
\hline \multicolumn{13}{|l|}{ Fisher-Weil } \\
\hline Bond maturities & $12,13^{*}$ & 101,171 & 144,225 & 12,53 & 98,269 & 123,285 & 12,79 & 85,242 & 139,332 & 12,15 & 71,86 & 126,276 \\
\hline \multirow[t]{2}{*}{ Return } & $8.1523 \%$ & $8.1518 \%$ & $8.1426 \%$ & $8.8667 \%$ & $10.7444 \%$ & $11.0852 \%$ & $14.7832 \%$ & $13.3512 \%$ & $12.8991 \%$ & $5.6248 \%$ & $6.8990 \%$ & $7.6099 \%$ \\
\hline & $(3.80 \mathrm{E}-06)$ & $(8.91 \mathrm{E}-10)$ & $(4.00 \mathrm{E}-09)$ & $(2.07 \mathrm{E}-12)$ & $(6.71 \mathrm{E}-10)$ & $(1.84 \mathrm{E}-08)$ & $(3.13 \mathrm{E}-12)$ & $(6.25 \mathrm{E}-11)$ & (1.08E-09) & $(1.22 \mathrm{E}-11)$ & $(5.11 \mathrm{E}-10)$ & $(1.77 \mathrm{E}-08)$ \\
\hline $\mathrm{AD}$ & $1.61 \mathrm{E}-08$ & $2.75 \mathrm{E}-05$ & $3.92 \mathrm{E}-05$ & $1.29 \mathrm{E}-07$ & $5.62 \mathrm{E}-05$ & $1.38 \mathrm{E}-04$ & $1.02 \mathrm{E}-07$ & $1.69 \mathrm{E}-05$ & $4.53 \mathrm{E}-05$ & $2.89 \mathrm{E}-08$ & $4.05 \mathrm{E}-06$ & $1.19 \mathrm{E}-04$ \\
\hline $\mathrm{RD}$ & $1.97 \mathrm{E}-07$ & $3.37 \mathrm{E}-04$ & $4.81 \mathrm{E}-04$ & $1.46 \mathrm{E}-06$ & $5.24 \mathrm{E}-04$ & $1.24 \mathrm{E}-03$ & $6.90 \mathrm{E}-07$ & $1.27 \mathrm{E}-04$ & $3.51 \mathrm{E}-04$ & $5.13 \mathrm{E}-07$ & $5.87 \mathrm{E}-05$ & $1.56 \mathrm{E}-03$ \\
\hline \multicolumn{13}{|l|}{ HJM } \\
\hline Bond maturities & 52,310 & 134,200 & 194,314 & 12,39 & 117,216 & 201,336 & 12,46 & 66,84 & 167,187 & 12,14 & 79,153 & 183,279 \\
\hline \multirow[t]{2}{*}{ Return } & $8.1593 \%$ & $8.1476 \%$ & $8.1496 \%$ & $8.8667 \%$ & $10.7447 \%$ & $11.1017 \%$ & $14.7832 \%$ & $13.3493 \%$ & $12.9006 \%$ & $5.6248 \%$ & $6.9002 \%$ & $7.6234 \%$ \\
\hline & (1.12E-09) & $(4.69 \mathrm{E}-10)$ & $(7.38 \mathrm{E}-11)$ & $(9.23 \mathrm{E}-13)$ & $(2.10 \mathrm{E}-10)$ & $(5.94 \mathrm{E}-11)$ & $(1.60 \mathrm{E}-12)$ & $(2.36 \mathrm{E}-10)$ & (1.94E-09) & $(7.35 \mathrm{E}-12)$ & $(1.63 \mathrm{E}-10)$ & $(8.54 \mathrm{E}-11)$ \\
\hline $\mathrm{AD}$ & 7.00E-05 & $6.98 \mathrm{E}-05$ & $3.03 \mathrm{E}-05$ & $5.05 \mathrm{E}-08$ & $5.90 \mathrm{E}-05$ & $2.75 \mathrm{E}-05$ & $4.41 \mathrm{E}-08$ & $1.44 \mathrm{E}-06$ & $3.00 \mathrm{E}-05$ & $1.05 \mathrm{E}-08$ & 7.98E-06 & $1.62 \mathrm{E}-05$ \\
\hline $\mathrm{RD}$ & 8.59E-04 & $8.56 \mathrm{E}-04$ & $3.71 \mathrm{E}-04$ & $5.69 \mathrm{E}-07$ & $5.49 \mathrm{E}-04$ & $2.47 \mathrm{E}-04$ & $2.98 \mathrm{E}-07$ & $1.08 \mathrm{E}-05$ & $2.33 \mathrm{E}-04$ & $1.87 \mathrm{E}-07$ & $1.16 \mathrm{E}-04$ & $2.13 \mathrm{E}-04$ \\
\hline
\end{tabular}

* Bullet portfolio 
Table 26 - Continued

Panel B

Duration and Convexity Matched Minimum M-Square Portfolios

\begin{tabular}{|c|c|c|c|c|c|c|c|c|c|c|c|c|}
\hline & \multicolumn{3}{|c|}{ (August, 1989) } & \multicolumn{3}{|c|}{$\begin{array}{c}\text { Increasing } \\
\text { (January, 1985) }\end{array}$} & \multicolumn{3}{|c|}{$\begin{array}{l}\text { Decreasing } \\
\text { (May, 1981) }\end{array}$} & \multicolumn{3}{|c|}{ (October, 1986) } \\
\hline & 1 year & 5 year & 10 year & 1 year & 5 year & 10 year & 1 year & 5 year & 10 year & 1 year & 5 year & 10 year \\
\hline \multicolumn{13}{|l|}{ Macaulay } \\
\hline Bond maturities & $12,13,14^{*}$ & $60,63,79$ & $120,140,182$ & $12,19,201$ & $60,64,66$ & $120,139,288$ & $12,20,140$ & $60,64,66$ & $120,131,321$ & $12,13,14^{*}$ & $60,61,66$ & $120,133,225$ \\
\hline \multirow[t]{2}{*}{ Return } & $8.1523 \%$ & $8.1567 \%$ & $8.1533 \%$ & $8.8665 \%$ & $10.7423 \%$ & $11.1006 \%$ & $14.7833 \%$ & $13.3503 \%$ & $12.8978 \%$ & $5.6249 \%$ & $6.9027 \%$ & $7.6241 \%$ \\
\hline & $(8.48 \mathrm{E}-06)$ & (388E-09) & (1.68E-07) & $(2.08 \mathrm{E}-11)$ & $(1.36 \mathrm{E}-08)$ & (3.33E-07) & $(1.98 \mathrm{E}-12)$ & (1.12E-09) & $(2.37 \mathrm{E}-07)$ & $(1.08 \mathrm{E}-05)$ & (1.40E-08) & (3.67E-07) \\
\hline $\mathrm{AD}$ & $9.69 \mathrm{E}-08$ & $2.13 \mathrm{E}-05$ & $6.83 \mathrm{E}-05$ & $1.64 \mathrm{E}-06$ & $3.47 \mathrm{E}-05$ & $1.58 \mathrm{E}-05$ & 7.49E-09 & $8.05 \mathrm{E}-06$ & $5.83 \mathrm{E}-05$ & $1.79 \mathrm{E}-07$ & $3.26 \mathrm{E}-05$ & $2.35 \mathrm{E}-05$ \\
\hline $\mathrm{RD}$ & $1.19 \mathrm{E}-06$ & $2.61 \mathrm{E}-04$ & $8.38 \mathrm{E}-04$ & $1.85 \mathrm{E}-05$ & $3.23 \mathrm{E}-04$ & $1.42 \mathrm{E}-04$ & $5.07 \mathrm{E}-08$ & $6.03 \mathrm{E}-05$ & $4.52 \mathrm{E}-04$ & $3.19 \mathrm{E}-06$ & 4.73E-04 & $3.08 \mathrm{E}-04$ \\
\hline \multicolumn{13}{|l|}{ Fisher-Weil } \\
\hline Bond maturities & $12,13,14^{*}$ & $60,61,66$ & $12,134,175$ & $12,16,136$ & $60,64,66$ & $120,123,236$ & $12,18,50$ & $60,64,66$ & $120,139,288$ & $12,13,14 *$ & $60,64,84$ & $120,134,191$ \\
\hline \multirow[t]{2}{*}{ Return } & $8.1523 \%$ & $8.1562 \%$ & $8.1538 \%$ & $8.8666 \%$ & $10.7422 \%$ & $11.1001 \%$ & $14.7833 \%$ & $13.3503 \%$ & $12.9047 \%$ & $5.6249 \%$ & $6.8773 \%$ & $7.6232 \%$ \\
\hline & $(8.47 \mathrm{E}-06)$ & $(8.40 \mathrm{E}-09)$ & $(4.72 \mathrm{E}-06)$ & $(1.85 \mathrm{E}-11)$ & $(1.30 \mathrm{E}-08)$ & $(2.03 \mathrm{E}-07)$ & $(3.14 \mathrm{E}-13)$ & $(1.24 \mathrm{E}-09)$ & $(9.30 \mathrm{E}-07)$ & $(1.08 \mathrm{E}-05)$ & $(3.39 \mathrm{E}-06)$ & \\
\hline $\mathrm{AD}$ & $9.69 \mathrm{E}-08$ & $1.60 \mathrm{E}-05$ & 7.33E-05 & $1.00 \mathrm{E}-06$ & $3.44 \mathrm{E}-05$ & $1.06 \mathrm{E}-05$ & $4.01 \mathrm{E}-08$ & $8.18 \mathrm{E}-06$ & $1.11 \mathrm{E}-05$ & $1.79 \mathrm{E}-07$ & $2.21 \mathrm{E}-04$ & $1.46 \mathrm{E}-05$ \\
\hline $\mathrm{RD}$ & $1.19 \mathrm{E}-06$ & $1.96 \mathrm{E}-04$ & $9.00 \mathrm{E}-04$ & $1.13 \mathrm{E}-05$ & $3.21 \mathrm{E}-04$ & $9.55 \mathrm{E}-04$ & $2.71 \mathrm{E}-07$ & $6.12 \mathrm{E}-05$ & $8.60 \mathrm{E}-05$ & 3.19E-06 & $3.20 \mathrm{E}-03$ & $1.92 \mathrm{E}-04$ \\
\hline \multicolumn{13}{|l|}{ HJM } \\
\hline Bond maturities & $12,13,14^{*}$ & $60,67,72$ & $120,130,150$ & $12,24,308$ & $60,66,71$ & $120,121,155$ & $12,20,56$ & $60,66,71$ & $120,121,155$ & $12,13,14 *$ & $60,61,66$ & $120,121,144$ \\
\hline \multirow[t]{2}{*}{ Return } & $8.1523 \%$ & $8.1544 \%$ & $8.1487 \%$ & $8.8666 \%$ & $10.7388 \%$ & $11.0993 \%$ & $14.7832 \%$ & $13.3495 \%$ & $12.9037 \%$ & $5.6248 \%$ & $6.8995 \%$ & $7.6218 \%$ \\
\hline & $(7.37 \mathrm{E}-06)$ & $(1.28 \mathrm{E}-10)$ & $(1.25 \mathrm{E}-07)$ & $(1.00 \mathrm{E}-14)$ & $(2.42 \mathrm{E}-10)$ & $(7.11 \mathrm{E}-10)$ & $(1.70 \mathrm{E}-15)$ & $(1.38 \mathrm{E}-09)$ & $(4.23 \mathrm{E}-10)$ & $(9.12 \mathrm{E}-06)$ & $(2.15 \mathrm{E}-11)$ & $(1.87 \mathrm{E}-10)$ \\
\hline $\mathrm{AD}$ & $5.24 \mathrm{E}-10$ & $1.85 \mathrm{E}-06$ & $2.20 \mathrm{E}-05$ & $2.76 \mathrm{E}-07$ & 2.09E-07 & $2.68 \mathrm{E}-06$ & $1.85 \mathrm{E}-09$ & $2.97 \mathrm{E}-07$ & $1.04 \mathrm{E}-06$ & $1.27 \mathrm{E}-09$ & $6.69 \mathrm{E}-07$ & $1.49 \mathrm{E}-06$ \\
\hline $\mathrm{RD}$ & $6.43 \mathrm{E}-09$ & $2.27 \mathrm{E}-05$ & $2.70 \mathrm{E}-04$ & $3.11 \mathrm{E}-06$ & $1.95 \mathrm{E}-06$ & $2.41 \mathrm{E}-05$ & $1.25 \mathrm{E}-08$ & $2.22 \mathrm{E}-06$ & $8.06 \mathrm{E}-06$ & $2.26 \mathrm{E}-08$ & $9.69 \mathrm{E}-06$ & $1.95 \mathrm{E}-05$ \\
\hline
\end{tabular}

* Bullet portfolio 
Bullet portfolios are the minimum M-square portfolios for the one-year holding period under a flat initial yield curve when we use traditional risk measures. For short to medium holding periods, bullet and barbell portfolios have closer returns to the target than minimum M-square portfolios. In this respect, a risk manager can prefer bullet and barbell portfolio strategies to minimizing M-squares of duration-matched portfolios.

Under this HJM framework, the volatility decay factor is estimated as negative. Therefore, volatility increases with the holding period. The time-to-payment variance also increases with the holding period. In these respects, we should observe higher M-squares for long holding periods. In other words, portfolio returns should be closer to the target as holding period decreases and vice versa. When we analyze Table 26, Panel A, we confirm these expectations. Irrespective of the risk measure used for duration matching, the deviation of minimum M-square portfolio returns from the target yield increases with the holding period.

Table 26, Panel B shows the results on the performances of duration and convexity matched minimum M-square portfolios. All minimum M-square portfolios are successful according to both active and passive portfolio management irrespective of the risk measures used for immunization. Matching with both duration and convexity improves the immunization performance marginally since all portfolios already showed to perform well with the duration matching strategy.

There are two portfolios that have the closest returns to the target. These are formed by traditional risk measures for the one-year holding period under a decreasing initial yield curve. There are six bullet portfolios that are minimum M-square portfolios. These bullet portfolios are formed by traditional and HJM risk measures for a one-year holding period under flat and humped initial yield curves. When we compare the performances of minimum M-square portfolios to those of bullet and barbell portfolios, we find that minimum M-square portfolios have closer returns to the target for short to medium holding periods. This is exactly the opposite of what we found with the duration matched minimum M-square portfolios. A possible explanation of these observations is in the nature of the immunization strategy. 
When a duration matching strategy is used, the nonlinear relation between bond price and yield change is not captured. This is more apparent when volatility is high. Under the humped volatility HJM framework, volatility is high for shorter holding periods. In this respect, the duration matching strategy may not be able to capture the nonlinearity for short to medium holding periods. Minimizing M-square is expected to reduce the effects of nonparallel shifts. Even though the shifts are parallel, if we observe large shifts, the convexity adjustment is still important. In this respect, when the duration and convexity matching strategy is used, minimizing M-square leads to closer returns to the target than bullet and barbell portfolios. With the duration matching strategy, bullet portfolios are expected to have close returns to the target. For short holding periods, term to maturity is the most important determinant of the duration, and bullet portfolios have closer times to maturity to the target. All of these effects can be possible explanations of why minimum M-square portfolios have closer returns to the target than bullet and barbell portfolios with the duration and convexity matching strategy but not with the duration matching strategy alone.

With the duration matching strategy, minimum M-square portfolios are successfully immunized under both active and passive management criteria for short holding periods. The duration matching strategy is also sufficient for minimum M-square portfolios when HJM risk measures are used. For traditional risk measures, however, using the duration and convexity matching strategy has significant benefits when passive portfolio management is pursued.

\subsection{Discussion of the Performance of Minimum M-Square Portfolios}

Minimum M-square portfolios are successfully immunized under all HJM frameworks with all risk measures when the aim is to have portfolio returns that are greater than or equal to the target yield minus five basis points. The immunization performances of the portfolios differ with respect to the risk measures, immunization strategy and holding periods when the aim is to have portfolio returns closer to the target.

For short holding periods, minimum M-square portfolios have returns within one basis point of the target with both duration matching and duration and convexity matching strategies using all risk measures. On the other hand, bullet portfolios mostly have the closest returns to the target for short holding periods. In these respects, for short holding periods, a 
risk manager can use the duration matching strategy with minimum M-square portfolios, but a better portfolio formation strategy is to use bullet portfolios for short holding periods.

For medium to long holding periods, minimum M-square portfolios are not as successful as they are for short holding periods. Also using the duration and convexity matching strategy rather than the duration matching strategy improves the immunization performances of minimum M-square portfolios significantly for medium to long holding periods with all risk measures. This is especially so with traditional risk measures under the passive portfolio management criterion. In this respect, the duration and convexity matching strategy has substantial benefits for medium to long holding periods.

Another important result is that the performance of minimum M-square portfolios is sensitive to the holding period and is not sensitive to the level of volatility. Minimum Msquare portfolios have better immunization performance for shorter holding periods even in the cases where shorter holding periods have higher volatility. In this respect, if there are rapid changes in the volatility in the market, an asset-liability manager may prefer to use minimum M-square portfolios with the duration and convexity matching strategy. Determining the correct risk measure is still important, but the performances of traditional risk measures are also good with minimum M-square portfolios. This also suggests that minimizing M-square reduces the effects of non-parallel shifts in the yield curve when traditional risk measures are used. 


\section{CHAPTER 10}

\section{CONCLUSION}

The most widely cited traditional risk measures are still the duration measures of Macaulay and Fisher-Weil and their corresponding convexity measures. Another traditional risk measure is M-square, which is designed to reduce the stochastic process risk that arises from non-parallel shifts in the yield curve. Since the introduction of these risk measures, we observe a rapid development in term structure models and the risk measures derived from these models. In theory, traditional risk measures are generally more restrictive than their complex counterparts that are derived from specific term structure models. Empirical evidence on the performance of traditional interest rate risk measures, however, is mixed. Some of the empirical studies find that traditional measures perform at least as well as their more complex counterparts, and some others find immunization strategies based on traditional measures not worthwhile.

Term structure models can be categorized as equilibrium and arbitrage-free models. One of the most popular arbitrage-free term structure models is the Heath-Jarrow-Morton (HJM) (1992), model. HJM is widely used in practice and the risk measures implied by these HJM models can be beneficial for immunization purposes. The limitations of HJM come from its complexity, the cost of implementation and the fact that it has non-Markovian spot and forward rates under certain volatility structures.

This study compares the immunization performance of traditional risk measures with the immunization performance of the risk measures derived from the selected HJM models. Two different immunization strategies are considered: Duration matching and duration and convexity matching strategies. When the shift in the yield curve is infinitesimal, assuming a linear relation between bond price and yield change is reasonable. Therefore, a duration matching strategy is an appropriate immunization strategy for small changes in the yield curve. If yield curve shifts are large, however, matching with convexity in addition to duration takes into account the nonlinear relation between bond price and yield curve change. 
Therefore, if yield curve shifts are large, duration and convexity matching should be a more appropriate immunization strategy.

Three different criteria are used to assess the immunization performance of portfolios that are duration matched or duration and convexity matched with traditional and HJM risk measures. The first criterion corresponds to active portfolio management. In active portfolio management, the aim of an asset-liability manager is to ensure that assets will have holding period returns equal to or greater than those of liabilities. In this respect, we consider a portfolio successfully immunized if the holding period return of the portfolio is greater than or equal to the target yield minus five basis points. The other two criteria correspond to passive portfolio management. Under passive portfolio management, the aim of an assetliability manager is to ensure that asset portfolio returns are as close as possible to the returns of the liability portfolio. To assess the immunization performance with respect to passive portfolio management, we determine if portfolio returns are within 100 basis points and one basis point of the target yield.

Four different one-factor HJM models are considered in the study: Constant, constant decay, exponential decay, and humped volatility. The coupon rates of Treasury securities, initial forward rates and their volatilities are inputs from real data. Using these inputs, the study is carried out by Monte-Carlo simulation. The evolution of forward rates is generated using the selected one-factor HJM models. Therefore, the benchmark risk measures are the ones determined by the specific one-factor HJM models.

Four initial yield curve shapes, - flat, increasing, decreasing, and humped-, and three holding periods, - one, five and ten years-, are considered with both duration matching and duration and convexity matching strategies. For each of these cases, one bullet, one barbell, one minimum M-Square and 100 random portfolios are formed with traditional as well as HJM risk measures.

The results on the performance of duration-matched portfolios suggest that with all HJM models considered in the study, traditional duration measures perform as well as their HJM counterparts when forward rate volatilities are low. This result is expected, since when there is low volatility, the relationship between the change in the bond price and the yield is approximately linear. Therefore, a duration matching strategy should provide successful 
immunization. When forward rate volatilities are high, however, traditional duration measures under-perform their HJM counterparts dramatically under the active portfolio management criterion. Under all HJM frameworks except the humped volatility HJM framework, the number of successfully immunized portfolios formed by HJM risk measures is at least 50\% more than those formed by traditional risk measures when the forward rate volatilities are high. Under the humped volatility HJM framework, the immunization performances of traditional and HJM risk measures are similar to each other with the duration matching strategy.

There may be several reasons that explain the underperformance of traditional risk measures when forward rate volatilities are high. One of the reasons may be that traditional duration measures are poor risk measures and therefore are not able to adequately capture the interest rate risk. Alternatively, the duration matching strategy may not be a good immunization strategy when forward rate volatilities are high. When volatilities are high, shifts in the yield curve are more likely to be non-infinitesimal. Therefore, assuming a linear relation between bond price and yield curve change and using the duration matching strategy may lead to under-performance of these risk measures. In this respect, we analyze the performance of these risk measures using the duration and convexity matching strategy. If significant underperformance of the traditional risk measures with active portfolio management is persistent, then it would suggest that using HJM risk measures over their traditional counterparts has significant benefits.

When the duration and convexity matching strategy is used, under the active portfolio management criterion, the performances of all risk measures are about the same or increase over the segments of the yield curve with high forward rate volatilities. The performances of traditional risk measures are about the same or slightly worse for the parts of the yield curve with low forward rate volatilities. This slight deterioration is not observed with HJM risk measures. Overall, the underperformance of traditional risk measures observed with the duration matching strategy decreases significantly when the duration and convexity matching strategy is used. The number of successfully immunized portfolios obtained using HJM risk measures is at most $33 \%$ more than the number of successfully immunized portfolios obtained using traditional risk measures. Generally, the difference between the number of 
successfully immunized portfolios obtained using HJM and traditional risk measures is less than $20 \%$ of the total portfolios considered. In these respects, the duration and convexity matching strategy improves the performance of traditional risk measures significantly. The performance improvement is not as drastic for HJM measures. With HJM measures, the improved performance is observed for long holding periods and not necessarily for high volatility segments of the yield curve. Under the humped volatility HJM framework, irrespective of the risk measures, the duration and convexity matching strategy does not lead to any improvement in the immunization performance.

Under the passive portfolio management criterion, when the duration matching strategy is used, the performances of HJM and traditional duration measures are similar. Almost all portfolios are within 100 basis points of the target, but at most $28 \%$ of the portfolios are within one basis point of the target yield under all HJM frameworks except the humped volatility HJM framework. Under the humped volatility HJM framework, a range of $30 \%$ to $94 \%$ of the portfolios have returns within one basis point of the target yield with both traditional and HJM duration measures. In this respect, an asset-liability manager pursuing passive portfolio management can prefer traditional risk measures to HJM risk measures due to their relative simplicity.

Under the passive portfolio management criterion, the duration and convexity matching strategy improves the immunization performance of HJM risk measures significantly. The improvement in the immunization performance of traditional risk measures, however, is not guaranteed. The performance of traditional risk measures improves with the duration and convexity matching strategy under constant and exponential decay volatility HJM frameworks but not under constant decay and humped volatility HJM frameworks. In fact, under constant decay and humped volatility HJM frameworks, the performance of traditional risk measures slightly deteriorates with the duration and convexity matching strategy. This result suggests that unless we know the correct volatility structure and adjust for the convexity accordingly, adding a second order term to the Taylor series expansion of the bond price around its yield does not guarantee a better approximation. In this respect, under the passive portfolio management criterion, determining the correct interest rate risk measure is more important than the selection of an immunization strategy. Also this result suggests 
that asset-liability managers pursuing passive portfolio management are more prone to interest rate risk than managers pursuing active portfolio management. Unless the correct interest rate risk measure is used, the duration and convexity matching strategy can worsen the performance of portfolios under the passive portfolio management criterion, whereas it generally improves the immunization performance under the active portfolio management criterion.

In these respects, an asset-liability manager who pursues passive portfolio management can use the duration matching strategy when dealing with the low volatility segments of the yield curve and if the correct interest rate risk measure cannot be specified. Under the active portfolio management criterion, if dealing with the whole yield curve or with the high volatility segments, the manager must adjust for convexity and use the duration and convexity matching strategy if the aim is to generate returns at least equal to those of the liabilities. Under the passive portfolio management criterion, determining the most accurate interest rate risk measure is more important than the immunization strategy since the duration and convexity matching strategy can worsen the immunization performance rather than improve it when a less accurate risk measure is used.

Using traditional risk measures versus HJM counterparts is a trade-off. Traditional risk measures are easy to implement and use. There is no need to invest much in human and computer resources. On the other hand, HJM risk measures require the implementation and calibration of specific HJM models. A significant investment in human and computer resources is necessary. If a firm decides to use one of the HJM measures considered in this study, it can improve the immunization performance of an active portfolio management by an uncertain $10 \%$ to $20 \%$ on average. It is possible, however, that it may not benefit from any improvement and even end up with underperformance. Under the passive portfolio management criterion, when the duration matching strategy is used, there is no benefit from HJM risk measures over traditional risk measures. Also, with the duration and convexity matching strategy, unless the HJM risk measure is the correct risk measure given the empirical data, there may be no improvement in immunization performance.

In this study, the results are strongly biased in the favor of the HJM risk measures. By design, the selected HJM models are the right models and their corresponding risk measures 
are the benchmarks. In the real world that may not be the case and, there will be model risk as well as estimation- and implementation-related problems. These factors can lead to similar performance or under-performance of these HJM risk measures compared to their traditional counterparts. In fact, this is what most empirical studies have found. Also, this finding could partially explain why practitioners continue to use duration and convexity even though the state of knowledge of term structure modeling has advanced significantly over the years.

Using the duration matching strategy versus the duration and convexity matching strategy is also a tradeoff. Under the active portfolio management criterion, matching with duration and convexity generally improves the immunization performance of the portfolios, especially with traditional risk measures. Under the passive portfolio management criterion, however, improved performance with the duration and convexity matching strategy is not guaranteed unless the correct interest rate risk measure is used. With real data, specifying the correct interest rate risk measure is nearly impossible, since the literature to date does not suggest a preference for one measure over another. In this respect, asset-liability managers who follow passive portfolio management are more prone to interest rate risk than those who follow active portfolio management. The duration matching strategy could yield better results than the duration-convexity matching strategy for these asset-liability managers unless they are sure that the selected interest rate risk measure is accurate with empirical data.

Portfolio formation strategies can also affect immunization performance. In these respects, for each scenario considered in the study, we form bullet, barbell and random portfolios. We find that for short holding periods, regardless of the risk measure and the HJM framework, bullet portfolios generally provide returns closest to the target yield. For medium to long holding periods, random portfolios are as good as bullet and barbell portfolios. When the duration and convexity matching strategy is used, both bullet and barbell portfolios produce returns within one basis point of the target yield under all HJM frameworks with both traditional and HJM risk measures. These results suggest that, for short holding periods, bullet portfolios are preferred to alternative portfolio formation strategies irrespective of the risk measure employed. For medium to long holding periods, however, we cannot favor one portfolio formation strategy over another. This result shows that when we adjust for both duration and convexity, the yield advantage of the bullet portfolio is offset by the convexity 
advantage of the barbell portfolio. For medium to long holding periods, however, immunization performance is not significantly affected by the portfolio formation strategy. Other factors such as the immunization strategy and risk measure seem to be more important.

We also analyze the performance of minimum M-square portfolios. M-square measures the time-to-payment variance of the bond price around duration. Minimizing the Msquare of duration matched or duration and convexity matched portfolios reduces the variance around duration. Therefore, minimum M-square portfolios should improve the immunization performance if yield curve shifts are not captured by the duration and convexity measures. We do not expect to observe significant improvement in the immunization performance of HJM risk measures by minimizing the $\mathrm{M}$-squares of the portfolios, because the forward rates are simulated according to the selected HJM models and therefore, the HJM risk measures should fully capture the yield curve shifts.

Our results suggest that, according to the active portfolio management criterion, all minimum M-square portfolios are successfully immunized with HJM as well as traditional risk measures. Under the passive portfolio management criterion, minimum M-square portfolios have returns within one basis point of the target yield for short holding periods with both duration, and duration-convexity matching strategies. When the duration matching strategy is used, the performance of these portfolios deteriorates irrespective of the volatility level when the holding period increases. Under the constant decay HJM framework, the volatilities are high for the short term and decreases with the time to maturity. Nevertheless, we observe that minimum M-square portfolios have closest returns to the target for short holding periods. Their performances deteriorate for long holding periods although the volatility levels are relatively low. In this respect, minimum M-square portfolios are more sensitive to the holding period rather than the volatility level. Therefore, these portfolios can be preferred in the markets when volatilities change frequently. The duration and convexity matching strategy improves the immunization performance of minimum M-square portfolios for medium to long holding periods. The portfolios that are matched with HJM risk measures, however, have closer returns to the target for long holding period than the portfolios matched with traditional risk measures. In this respect, although the immunization strategy is important, so is the risk measure, especially under the passive portfolio management criterion. 
In most of the cases, minimum M-square portfolios are not the ones with the closest returns to the target yield. With the duration matching strategy, bullet portfolios produce closer returns to the target than minimum M-square portfolios for short holding periods. The times to maturity of the bonds in bullet portfolios are generally closer to those of the target bond. In this respect, this result suggests that time to maturity is the most important determinant of duration for short holding periods. The immunization performances of bullet, barbell, and minimum M-square portfolios are similar to each other for medium to long holding periods.

The comparison of HJM risk measures with their simpler counterparts indicates the strengths and weaknesses of traditional risk measures. The study also provides a better understanding of the contribution of HJM models to immunization, and whether the additional complexity is worthwhile. HJM models are widely used in the financial world. If HJM models are the best approximations of how interest rates behave, then to know the conditions under which traditional risk measures and certain immunization strategies hold is beneficial for interest rate risk management purposes. The study also compares the performances of alternative immunization and portfolio formation strategies under both active and passive portfolio management criteria. These comparisons shed light on the advantages and disadvantages of particular strategies with respect to each other.

There are several limitations of this study. First of all, only one-factor HJM models are considered. Therefore, the limitations of one-factor models apply. One random factor leads to perfect correlation among bonds of different maturities, which is not the case in the real world. Additionally, the volatility structures imposed by the HJM models are a very limited subset of the available volatility structures. Although the study applies some of the most widely used volatility structures to the one-factor HJM models, considering alternative volatility structures and allowing more than one random factor can be a worthwhile extension. Also, instead of using a specific volatility structure, the empirical term structure of volatilities can be imposed on the HJM models. Another limitation of the study is that only option and default free bonds are considered, whereas most corporate bonds have options and default risk. Extending the study for default risk and option characteristics would be a challenging task but a valuable contribution to the risk management literature. 


\section{REFERENCES}

Amin, K. L., and A. Morton. "Implied Volatility Functions in Arbitrage Free Term Structure Models." Journal of Financial Economics, 1994, Vol. 35, pp. 141-180.

Amin, K. L., and V. K. Ng. "Inferring Future Volatility from the Information in Implied Volatility in Eurodollar Options: A New Approach." Review of Financial Studies, Summer 1997, Vol. 10, No. 2, pp. 333-367.

Atkinson, A. C., and M. C. Pearce. "The Computer Generation of Beta, Gamma, and Normal Random Variables.” Journal of Royal Statistics Association, 1976, A139: 431-448.

Au, K. T., and D. C. Thurston. "A New Class of Duration Measures." Economics Letters, 1995, Vol. 47, pp. 371-375.

Barber, J. R. "Bond Immunization for Affine Term Structures." Financial Review, 1999, Vol. 34, pp. 127-140.

Baxter, M., and A. Rennie. Financial Calculus: An Introduction to Derivatives Pricing, Cambridge University Press, Cambridge, 1996.

Bierwag, G. O. "Immunization, Duration and the Term Structure of Interest Rates." Journal of Financial and Quantitative Analysis, December 1977, pp. 725-741.

Bierwag, G. O. "Dynamic Portfolio Immunization Policies.” Journal of Banking and Finance, April 1979, pp. 23-42.

Bierwag, G. O. "Bond Returns, Discrete Stochastic Processes, and Duration." Journal of Financial Research, Fall 1987, Vol. 10, No. 3, pp. 191-209.

Bierwag, G. O. "The Ho-Lee Binomial Stochastic Process and Duration.” Journal of Fixed Income, September 1996, pp. 76-87.

Bierwag, G. G., I. Fooladi, and G. S. Roberts. "Designing an Immunized Portfolio: Is MSquared the Key?” Journal of Banking and Finance, 1993, Vol. 17, pp. 1147-1170.

Bierwag, G. O., and G. G. Kaufman. "Coping with the Risk of Interest Rate Fluctuation: A Note.” Journal of Business, July 1977, pp. 364-370.

Bierwag, G. O., G. G. Kaufman, and C. M. Latta. "Bond Portfolio Immunization: Test of Maturity, One- and Two-Factor Duration Matching Strategies." Financial Review, May 1987, Vol. 22, No. 2, pp. 203-219. 
Bierwag, G. O., G. G. Kaufman, R. Schweitzer, and A. Toevs. "The Art of Risk Management in Bond Portfolios.” Journal of Portfolio Management, Spring 1981, pp. 27-36.

Bierwag, G. O., G. G. Kaufman, and A. Toevs. "Immunization Strategies for Funding Multiple Liabilities." Journal of Financial and Quantitative Analysis, 1983, Vol. 18, pp. 113123.

Bierwag, G. O., and C. Khang. "An Immunization Strategy is a Minimax Strategy.” Journal of Finance, 1979, Vol. 34, pp. 389-399.

Björk, T., Kabanov Y., and W. Runggaldier. "Bond Market Structure in the Presence of Marked Point Processes." Mathematical Finance, 1997, Vol. 7, No. 2, pp. 211-239.

Black, F., and P. Karasinski. "Bond and Option Pricing When Short Rates are Lognormal." Financial Analyst Journal, July-August 1991, pp. 52-59.

Bliss, R. "Testing Term Structure Estimation Methods." Advances in Futures and Options Research, 1997, Vol. 9, pp. 197-231.

Bliss, R., and P. Ritchken. "Empirical Tests of Two State-Variable Heath, Jarrow, Morton Models." Journal of Money, Credit and Banking, 1996, Vol. 28, pp. 452-476.

Box, G. E. P., and M. E. Muller. "A Note on the Generation of Random Normal Deviates." Annals of Mathematical Statistics, 1958, Vol. 29, pp. 610-611.

Boyle, P., M. Broadie, and P. Glasserman. "Monte Carlo Methods for Security Pricing." Journal of Economic Dynamics and Control, 1997, Vol. 21, pp. 1267-1321.

Brennan, M. J., and E. S. Schwartz. "Duration, Bond Pricing, and Portfolio Management." in Innovations in Bond Portfolio Management: Duration Analysis and Immunization, Kaufman G. G., G. O. Bierwag, and A. Toevs, editors, Greenwich, CT: JAI Press, 1983.

Bühler, W., Uhrig, M., Walter, U., and T. Weber. “An Empirical Comparison of Forwardand Spot-Rate Models for Valuing Interest-Rate Options." Journal of Finance, 1999, Vol. 54, No. 1, pp. 261-305

Caverhill, A. P. "When is the Short Rate Markovian?" Mathematical Finance, October 1994, Vol. 4, No. 4, pp. 305-312.

Chambers, D. R., and W. T. Carleton. "A More General Duration Approach.” In Research and Finance, A. H. Chen edition, JAI Press, 1981.

Chambers, D. R., W. T. Carleton, and R. W. McEnally. "Immunizing Default-Free Bond Portfolios with a Duration Vector." Journal of Financial and Quantitative Analysis, March 1988, Vol. 23, No. 1, pp. 89-104. 
Chan, K. C., Karolyi, G. A., Longstaff, F. A., and A. B. Sanders. "An Empirical Comparison of Alternative Models of the Short-Term Interest Rate." Journal of Finance, July 1992, Vol. 47, No. 3, pp. 1209-1227.

Chance, D. M., and J. V. Jordan. "Duration, Convexity, and Time as Components of Bond Returns.” Journal of Fixed Income, September 1996, pp. 88-96.

Chapman, D. A., and N. D. Pearson. "Recent Advances in Estimating Term-Structure Models." Financial Analysts Journal, July/August 2001, Vol. 57, No. 4, pp. 77-95.

Christensen, P. O., and B. G. Sorensen. "Duration, Convexity, and Time Value." Journal of Portfolio Management, Winter 1994, pp. 51-60.

Colwell, D. B. "Forward Measures in a Ho and Lee Jump Diffusion Model." Working Paper, University of New South Wales, Australia, 2001.

Cooper, I. A. "Asset Values, Interest rate Changes, and Duration." Journal of Financial and Quantitative Analysis, December 1977, Vol. 12, pp. 701-723.

Courtadon, G. "The Pricing of Options on Default-Free Bonds." Journal of Financial and Quantitative Analysis, 1982, Vol. 17, pp. 75-100.

Cox, J., J. E. Ingersoll, and S. A. Ross. "Duration and Measurement of Basis Risk." Journal of Business, January 1979, pp. 57-61.

Cox, J., J. E. Ingersoll, and S. A. Ross. "A Theory of the Term Structure of Interest Rates." Ecnometrica, March 1985, Vol. 53, pp. 385-407.

Dai, Q., and K. J. Singleton. "Specification Analysis of Affine Term Structure Models." Journal of Finance (forthcoming), 2000.

Duffie, D. Dynamic Asset Pricing Theory, 2nd edition, Princeton University Press, Princeton, New Jersey, 1996.

Duffie, D., and P. Glynn. "Efficient Monte Carlo Simulation of Security Prices." Annals of Applied Probability, Vol. 5, No. 4, pp. 897-905.

Duanmu, Z. First Passage Time Density Approach to Pricing Barrier Options and Monte Carlo Simulation of HJM Interest Rate Model, Ph.D. Dissertation, Cornell University, 1994.

Durand, D. "Basic Yields of Corporate Bonds 1900-42." Technical Paper No. 3, New York, National Bureau of Economic Research, 1942. 
Fabozzi, F. J. Bond Markets, Analyses and Strategies, Third edition, Prentice Hall, New Jersey, 1996.

Fabozzi, F. J., and G. Fong. Fixed Income Portfolio Management, Irwin Professional Publishing, 1994.

Fama, E., and R. Bliss. "The Information in Long-Maturity Forward Rates." American Economic Review, 1987, Vol. 77, pp. 680-692.

Fisher, L., and R. L. Weil. "Coping with the Risk of Market Rate Fluctuations: Returns to Bondholders from Naive and Optimal Strategies." Journal of Business, October 1971, Vol. 44, pp. 408-431.

Flesaker, B. "Testing the Heath-Jarrow-Morton/Ho-Lee Model of Interest Rate Contingent Claims Pricing." Journal of Financial and Quantitative Analysis, December 1993, Vol. 28, No. 4, pp. 483-495.

Fong, H. G., and O. Vasicek. "Return Maximization for Immunized Portfolios." in Innovations in Bond Portfolio Management: Duration Analysis and Immunization, Kaufman G. G., G. O. Bierwag, and A. Toevs, editors, Greenwich, CT: JAI Press, 1983.

Fruhwirth, M. "The Heath/Jarrow/Morton Duration and Convexity - A Generalized Approach." Working Paper, Vienna University of Economics and Business Administration, Vienna, Austria, June 2001.

Fuller, J. R., and J. W, Settle. "Determinants of Duration and Bond Volatility." Journal of Portfolio Management, Summer 1994, pp. 66-72.

Goldstein, R. “The Term Structure of Interest Rates as a Random Field.” Review of Financial Studies, Summer 2000, Vol. 13, No. 2, pp. 365-384.

Gultekin, N. B., and R. J. Rogalski. "Alternative Duration Specifications and the Measurement of Basis Risk: Empirical Tests." Journal of Business, 1984, Vol. 57, No. 2, pp. 241-264.

Gupta, A., and M. G. Subrahmanyam. "An Empirical Examination of the Convexity Bias in the Pricing of Interest Rate Swaps.” Journal of Financial Economics, 2000, Vol. 55, pp. 239279 .

Heath, D., R. Jarrow, and A. Morton. “Contingent Claim Valuation with a Random Evolution of Interest Rates" Review of Futures Markets, 1990, Vol.9, pp. 54-78.

Heath, D., R. Jarrow, and A. Morton. "Bond Pricing and the Term Structure of Interest Rates: A Discrete Time Approximation." Journal of Financial and Quantitative Analysis, December 1990, Vol. 25, No. 4, pp. 419-440. 
Heath, D., R. Jarrow, and A. Morton. "Bond Pricing and the Term Structure of Interest Rates: A New Methodology for Contingent Claims Valuation.” Econometrica, 1992, Vol. 60, No. 1, pp. 77-105.

Hicks, J. R. Value and Capital, Claderon Press, Oxford, 1939.

Ho, T. S. Y. "Evolution of Interest Rate Models: A Comparison." Journal of Derivatives, Summer 1995, pp. 9-20.

Ho, L., Cadle, J. and M. Theobald. "Estimation and Hedging with a One-Factor HeathJarrow-Morton Model.” Journal of Derivatives, Summer 2001, pp. 49-61.

Ho, T. S. Y., and S. Lee. "Term Structure Movements and Pricing Interest Rate Contingent Claims." Journal of Finance, December 1986, Vol. 41, No. 5, pp. 1011-1029.

Hull, J. C., and A. D. White. "Pricing Interest-Rate-Derivative Securities." Review of Financial Studies, 1990, Vol. 3, No. 4, pp. 573-592.

Hull, J. C., and A. D. White. "Bond Option Pricing Based on a Model for the Evolution of Bond Prices." Advances in Futures and Options Research, 1993, Vol. 6, pp. 1-13.

Hull, J., and A. White. "Numerical Procedures for Implementing Term Structure Models 1: Single-Factor Models.” Journal of Derivatives, Fall 1994, pp. 7-16.

Hull, J. C., and A. D. White. "A Note on the Models of Hull and White for Pricing Options on the Term Structure." Journal of Fixed Income, September 1995, pp. 97-102.

Ilmanen, A. "How Well Does Duration Measure Interest Rate Risk?" Journal of Fixed Income, March 1992, pp. 43-51.

Ilmanen, A., and R. Iwanovski. "Dynamics of the Shape of the Yield Curve." Journal of Fixed Income, September 1997, pp. 47-60.

Ingersoll, J. E., J. Skelton, and R. L. Weil. "Duration Forty Years Later.” Journal of Financial and Quantitative Analysis, November 1978, pp. 621-650.

Inui, K., and M. Kijima. "A Markovian Framework in Multi-Factor Heath-Jarrow-Morton Models." Journal of Financial and Quantitative Analysis, September 1998, Vol. 33, No. 3, pp. 423-440.

James, J., and N. Webber. Interest Rate Modelling, John Wiley \& Sons Ltd., 2000.

Jarrow, R. A. Modeling Interest Rate Securities and Interest Rate Options, McGraw-Hill Companies Inc., 1996. 
Jarrow, R. A., and S. M. Turnbull. "Delta, Gamma, and Bucket Hedging of Interest Rate Derivatives." Applied Mathematical Finance, Vol. 1, No. 1, pp. 21-48.

Jeffrey, A. "Single Factor Heath-Jarrow-Morton Term Structure Models Based on Markov Spot Interest Rate Dynamics." Journal of Financial and Quantitative Analysis, December 1995, Vol. 30, No. 4, pp. 619-642.

Jeffrey, A. "Duration, Convexity and Higher Order Hedging (Revisited)." Working Paper, Yale School of Management, November 2000.

Kennedy, D. P. "The Term Structure of Interest Rates as a Gaussian Random Field." Mathematical Finance, April 1994, Vol. 4, No. 3, pp. 247-258.

Kennedy, D. P. "Characterizing Gaussian Models of the Term Structure of Interest Rates." Mathematical Finance, April 1997, Vol. 7, No. 2, pp. 107-118.

Khang, C. "Bond Immunization When Short Term Interest Rates Fluctuate More than Long Term Rates." Journal of Financial and Quantitative Analysis, December 1979, pp. 10851090 .

Kinderman, A. J., and J. G. Ramage. "Computer Generation of Normal Random Variables." Journal of American Statistics Association, 1976, Vol. 71, pp. 893-896.

Kloeden, P. E., and E. Platen. Numerical Solution of Stochastic Different Equations, Springer-Verlang, 1992.

L'Ecuyer P. "Good Parameters and Implementations for Combined Multiple Recursive Random Number Generators.” Operations Research, 1999, Vol. 47, No. 1, pp. 159-164.

Law, A. M., and W. D. Kelton. Simulation Modeling and Analysis, Second Edition, McGrawHill, 1991.

Leibowitz, M. L. "Bond Immunization: A Procedure for Realizing Target Levels of Return: Bond Immunization - Part II: Portfolio Rebalancing; Bond Immunization Part III: The Yield Curve Case." Reprinted in Pros \& Cons of Immunization, edited by M. L. Leibowitz, Salomon Brothers Inc, 1980.

Li, A., Ritchken, P., and L. Sankarasubramanian. "Lattice Models for Pricing American Interest Rate Claims.” Journal of Finance, 1995, pp. 719-737.

Litterman, R., and J. Scheinkman. "Common Factors Affecting Bond Returns." Journal of Fixed Income, December 1991, Vol. 3, No. 3, pp. 54-61. 
Longstaff, F. A., and E. S. Schwartz. "Interest Rate Volatility and the Term Structure: A Two Factor General Equilibrium Model." Journal of Finance, 1992, Vol. 47, No. 4, pp. 12591282.

Macaulay, F. R. Some Theoretical Problems Suggested by the Movements of Interest Rates, Bond Yields and Stock Prices in the United States Since 1856. Columbia University Press, New York, 1939.

Maloney, K. J., and J. B. Yawitz. "Interest Rate Risk, Immunization, and Duration." Journal of Portfolio Management, Spring 1986, pp. 41-48.

Mann, S. V., and P. Ramanlal. "Duration and Convexity Measures When the Yield Curve Changes Shape.” Journal of Financial Engineering, 1998, Vol. 7, No. 1, pp. 35-58.

Marsaglia, G., and T. A. Bray. "A Convenient Method for Generating Random Variables." SIAM Review, 1964, Vol. 6, pp. 260-264.

Mattoo, M. "Why Hedge?" The Handbook of Interest Rate Risk Management, in J. C. Francis, and A. S. Wolf edition, Irwin Professional Publishing, 1994.

McCulloch, J. H. “An Estimate of Liquidity Premium.” Journal of Political Economy, February 1975, Vol. 83, No. 1, pp. 95-119.

McCulloch, J. H., and Kwon, H-C. "U.S. Term Structure Data, 1947-1991.” Working Paper, Ohio State University, 1993.

Mercurio F., and J. M. Moraleda. "An Analytically Tractable Interest Rate Model with Humped Volatility." European Journal of Operational Research, 2000, Vol. 120, pp. 205214.

Moraleda, J. M., and A. Pelsser. "Forward versus Spot Interest Rate Models of the Term Structure: An Empirical Comparison." Journal of Derivative, Spring 2000, pp. 9-21.

Munk, C. "Stochastic Duration and Fast Coupon Bond Option Pricing in Multi-Factor Models." Working Paper, Odense University, Denmark, 1998.

Musiela, M., Turnbull, S. M., and L. M. Wakeman. "Interest Rate Risk Management." Review of Futures Markets, 1993, Vol. 12, No. 1, pp. 221-261.

Nawalkha, S. K. "Closed-Form Solutions of Convexity and M-Square." Financial Analysts Journal, January-February 1990, pp. 75-77.

Nawalkha, S. K. "The Duration Vector: A Continuous-Time Extension of Default-Free Interest Rate Contingent Claims." Journal of Banking and Finance, 1995, Vol. 19, pp. 13591378. 
Nawalkha, S. K., and N. J. Lacey. "Generalized Solutions of Higher-Order Duration Measures.” Journal of Banking and Finance, 1990, Vol. 14, pp. 1143-1150.

Nelson, J., and S. Schaefer. "The Dynamics of the Term Structure and Alternative Immunization Strategies." in Innovations in Bond Portfolio Management: Duration Analysis and Immunization, Kaufman G. G., G. O. Bierwag, and A. Toevs, editors, Greenwich, CT: JAI Press, 1983.

Nelson, B. L., and B. W. Schmeiser. "Decomposition of Some Well-Known Variance Reduction Techniques.” Journal of Statistical and Computational Simulation, 1986, Vol. 23, pp. 183-209.

Odeh, R. E., and J. O. Evans. "The Percentage Points of the Normal Distribution." Applied Statistics, 1974, Vol. 23, pp. 96-97.

Pelsser, A. A. J. "A Tractable Yield-Curve Model that Guarantees Positive Interest Rates." Review of Derivatives Research, 1997, Vol. 1, pp. 269-284.

Press, W. H., S. A. Teukolsky, W. T. Vetterling, B. P. Flannery. Numerical Recipes in C: The Art of Scientific Computing, Second Edition, Cambridge University Press, 1992.

Prisman, E. Z., and M. R. Shores. "Duration Measures for Specific Term Structure Estimations and Applications to Bond Portfolio Immunization." Journal of Banking and Finance, 1988, Vol. 12, pp. 493-504.

Redington, F. M. "Review of the Principle of Life-Office Valuation." Journal of the Institute of Actuaries, 1952, Vol. 78, pp. 286-340.

Rebonato, R. Interest Rate Option Models, Second Edition, John Wiley and Sons, 1998.

Ritchken, P., and L. Sankarasubramanian. "Volatility Structures of Forward Rates and the Dynamics of the Term Structure." Mathematical Finance, January 1995, Vol. 5, No. 1, pp. 55-72.

Samuelson, P. A. "The Effects of Interest Rate Increases on the Banking System." American Economic Review, March 1945, pp. 16-27.

Santa-Clara, P., and D. Sornette. "The Dynamics of the Forward Interest Rate Curve with Stochastic String Shocks." Review of Financial Studies, Spring 2001, Vol. 14, No. 1, pp. 149185.

Winkelman, K. "Uses and Abuses of Duration and Convexity." Financial Analyst Journal, September-October 1989, pp. 72-75. 
Willmott, P. Derivatives: The Theory and Practice of Financial Engineering, John Wiley \& Sons, 1998.

Vasicek, O. A. "An Equilibrium Characterization of the Term Structure." Journal of Financial Economics, November 1977, Vol. 5, pp. 177-188.

Yan, H. "Dynamic Models of the Term Structure." Financial Analysts Journal, July/August 2001, pp. 60-76. 


\section{APPENDIX 1}

The Taylor's series expansion of a coupon bond price, $B(t, T)$, around its yield is given below. The dependence of $B(t, T)$ on $\mathrm{t}$ and $\mathrm{T}$ are suppressed since the immunization is instantaneous:

$$
\begin{aligned}
& B(y+\Delta y)-B(y)=\frac{\partial B(y)}{\partial y} \Delta y+\frac{1}{2} \frac{\partial^{2} B(y)}{\partial y^{2}}(\Delta y)^{2} \\
& B(y)=\sum_{i=1}^{n} C_{i} P\left(y, t, \tau_{i}\right)
\end{aligned}
$$

\section{Duration}

Similar to the coupon bond price, the $\mathrm{t}$ and $\mathrm{T}$ in the zero coupon bond price $P\left(y, t, \tau_{i}\right)$ are also suppressed in the below equations.

$$
\frac{\partial B(y)}{\partial y}=\sum_{i=1}^{n} C_{i} \frac{\partial P(y)}{\partial y}
$$

\section{a. Discrete Yield}

$$
P\left(y, t, \tau_{i}\right)=\frac{1}{(1+y)^{\left(\tau_{i}-t\right)}}
$$

By (A1.3) and (A1.4),

$$
\begin{aligned}
& \frac{\partial B(y)}{\partial y}=\sum_{i=1}^{n} \frac{-\left(\tau_{i}-t\right) C_{i}}{(1+y)(1+y)^{\left(\tau_{i}-t\right)}} \\
& \frac{\partial B(y)}{\partial y}=\sum_{i=1}^{n} \frac{-\left(\tau_{i}-t\right) C_{i} P\left(y, t, \tau_{i}\right)}{(1+y)}
\end{aligned}
$$

Therefore, the relation of duration, $D(t)$, and bond price is as follows: 
$\frac{\partial B(y)}{\partial y}=-D(t) \frac{1}{(1+y)} B(y)$

By (A1.6), the relation of modified duration $M D(t)$ and bond price is as follows:

$$
\frac{\partial B(y)}{\partial y}=-M D(t) B(y)
$$

\section{b. Continuous Yield}

$$
P\left(y, t, \tau_{i}\right)=e^{-y\left(\tau_{i}-t\right)}
$$

By (A1.3) and (A1.8),

$$
\begin{aligned}
& \frac{\partial B(y)}{\partial y}=\sum_{i=1}^{n}-\left(\tau_{i}-t\right) C_{i} e^{-y\left(\tau_{i}-t\right)} \\
& \frac{\partial B(y)}{\partial y}=\sum_{i=1}^{n}-\left(\tau_{i}-t\right) C_{i} P\left(y, t, \tau_{i}\right)
\end{aligned}
$$

Therefore, the relation of duration, $D(t)$, and bond price is as follows:

$$
\frac{\partial B(y)}{\partial y}=-D(t) B(y)
$$

As it is seen from the above equation there is no modification needed for the continuous duration measures. 


\section{Convexity}

$$
\begin{aligned}
& \operatorname{Conv}(t)=\frac{1}{B(y)} \frac{\partial^{2} B(y)}{\partial y^{2}} \\
& \frac{\partial^{2} B(y)}{\partial y^{2}}=\sum_{i=1}^{n} C_{i} \frac{\partial^{2} P(y)}{\partial y^{2}}
\end{aligned}
$$

\section{a. Discrete Yield}

By (A1.4) and (A1.12),

$$
\begin{aligned}
& \frac{\partial^{2} B(y)}{\partial y^{2}}=\sum_{i=1}^{n} \frac{-\left(\tau_{i}-t\right)\left(\tau_{i}-t+1\right) C_{i}}{(1+y)^{2}(1+y)^{\left(\tau_{i}-t\right)}} \\
& \frac{\partial^{2} B(y)}{\partial y^{2}}=\sum_{i=1}^{n} \frac{-\left(\tau_{i}-t\right)\left(\tau_{i}-t+1\right) C_{i} P\left(y, t, \tau_{i}\right)}{(1+y)^{2}}
\end{aligned}
$$

Therefore, the convexity with discrete yield is as follows:

$$
\operatorname{Conv}(t)=\frac{1}{B(t, T)} \sum_{i=1}^{n} \frac{\left(\tau_{i}-t\right)\left(\tau_{i}-t+1\right) C_{i} P\left(y, t, \tau_{i}\right)}{(1+y)^{2}}
$$

\section{b. Continuous Yield}

$$
\begin{aligned}
& \frac{\partial B(y)}{\partial y}=\sum_{i=1}^{n}-\left(\tau_{i}-t\right) C_{i} e^{-y(\tau-t)} \\
& \frac{\partial B(y)}{\partial y}=\sum_{i=1}^{n}-\left(\tau_{i}-t\right) C_{i} P\left(y, t, \tau_{i}\right)
\end{aligned}
$$

Therefore, the convexity with continuous yield is as follows:

$$
\operatorname{Conv}(t)=\frac{1}{B(t, T)} \sum_{i=1}^{n}\left(\tau_{i}-t\right)^{2} C_{i} P\left(y, t, \tau_{i}\right)
$$




\section{TECHNICAL APPENDIX 1}

\section{SELECTION OF INITIAL YIELD CURVES}

For simulation purposes, four initial yield curves are selected from the McCulloch and Kwon (1993) zero-coupon yield curve data. The yield curve information in this data set begins on January 1947 and ends on February 1991. The initial yield curves considered in this study are constant, increasing, decreasing and humped yield curves. One should note that these are the initial yield curves and will change during the simulation of the forward rate curves according to the selected one-factor HJM models. We choose these four different shapes for the initial yield curve mainly because the yield curves can be categorized into one of these shapes or the combination of these different shapes.

The selection process is carried out by an analysis of the yield curve plots. The initial yield curves of each month from January 1947 to February 1991 are plotted. These plots are then categorized into different groups. Four of these groups are flat, increasing, decreasing, and humped. The other group consists of yield curves that are combination of these shapes. The yield curves that cannot be categorized into one of the four shapes are in the other group. One should note that the ones chosen as flat, increasing, and decreasing are not perfectly fit the criteria but are the ones that are closest to these shapes among all the yield curves.

The yield curves that can be categorized as flat yield curve are the ones on November and December 1955, November and December 1964, November 1965, February 1967, February and August 1968, March 1969, January 1973, July and October 1974, December 1988, February, August, September and December 1989, and April 1990. After making a second pass on these yield curves, we choose August 1989 as our representative flat initial yield curve.

The yield curves that can be categorized as increasing are the ones on March and October 1951, June, July, August, September, October, November and December 1952, April, 1953, June

to October 1953, December 1953, February to May 1954, July 1954, September to November 1954, February, March and May 1958, September and October 1961, July 1962, March, August, October and December 1971, March and May 1972, February 1973, January, February and April 1975, June to October 1975, March 1976, May to August 1976, December 1976, February 1977, June to September 1977, November 1977, February 1984, January and May 1985. After making 
a second pass on these yield curves, we choose January 1985 as our representative increasing initial yield curve.

The yield curves that can be categorized as decreasing are the ones on August 1973, September to December 1979, October and November 1980, January and May 1981, and March 1982. After making a second pass on these yield curves, we choose May 1981 as our representative decreasing initial yield curve.

The yield curves that can be categorized as humped are the ones on May 1953, July 1955, March and April 1956, July to September 1956, November 1956, August and September 1957, April 1959, January 1960, November and December 1961, January 1962, April, August, October, December 1965, March and April 1966, July to September 1966, December 1966, June 1967, January, April, July, September and October 1968, July 1969, October to December 1969, May, October and December 1978, February and August 1979, July 1980, January, April, June and October 1983, March, April, June, July, September and November 1985, January 1986, April to December 1986, January to April 1987, June 1987, January, February 1990, May to July 1990, September to December 1990, and January 1991. After making a second pass on these yield curves, we choose October 1986 as our representative humped initial yield curve. 


\section{TECHNICAL APPENDIX 2}

\section{ESTIMATION OF VOLATILITY PARAMETERS OF THE SELECTED ONE-FACTOR HJM MODELS FOR THE INCREASING INITIAL YIELD CURVE OF JANUARY 1985}

The first step is to estimate the volatilities of different maturity forward rates on January 1985. Four years of historical monthly forward rates are used. Therefore, to estimate the volatilities of forward rates on January 1985, monthly forward rates from January 1981 to January 1985 are considered.

To estimate forward rate volatilities, three-factor principal component analysis (PCA) is used. Jarrow (1996), Rebonato (1996), Willmott (1998), and James and Weber (2000) ${ }^{1}$ suggest this method to estimate forward rate volatilities of HJM models. Litterman and Scheinkman (1991) and Chapman and Pearson (2001) find that three factors explain 99\% of variation in the yield curve, and the first factor alone can explain $83 \%$ to $90 \%$ of the variation. In this respect, we assume that a three-factor HJM model explains all the variation and therefore carry out a three-factor PCA. We take the first factor for our volatility estimates of forward rate volatilities since we are using one-factor HJM models and the first factor should explain more than $80 \%$ of the variation in the yield curve.

In the McCulloch and Kwon (1993) data set, monthly forward rates are on a continuously compounded basis. When these forward rates are discretized by Euler scheme, the forward rate equation for a three-factor HJM model is as follows:

$$
\widetilde{f}\left(t_{i+1}, T\right)=\widetilde{f}\left(t_{i}, T\right)+\alpha\left(t_{i}, T\right)\left(t_{i+1}-t_{i}\right)+\sum_{j=1}^{3} \sigma_{j}\left(t_{i}, T\right)\left(\tilde{W}_{j}\left(t_{i+1}\right)-\widetilde{W}_{j}\left(t_{i}\right)\right) \quad, \quad i=0,1, \ldots N
$$

In above equation, $\widetilde{f}\left(t_{i}, T\right)$ is the value of the approximation of $f\left(t_{i}, T\right)$ at the discretization time $t_{i}$. At time $t_{\mathrm{i}}$, the drift and the volatility functions of the forward rate are $\alpha\left(t_{i}, T\right)$ and $\sigma_{j}\left(t_{i}, T\right)$, respectively. Under the equivalent martingale measure, $\widetilde{W}_{j}\left(t_{i}\right)$ is a

\footnotetext{
${ }^{1}$ See Jarrow (1996), pp. 208-213; Rebonato (1996), pp. 51-74; Willmott (1998), pp. 504-507; James and Weber (2000), pp. 455-485.
} 
Wiener process. There are three random factors affecting the evolution of forward rates. Since monthly forward rates are used, the increment $t_{i+1}-t_{i}$ is taken as one month.

According to (TA1), the change in forward rates is distributed normally with a $3 \times 1$ mean vector and 3x3 variance-covariance matrix. In this respect, we first find the monthly change in the forward rates from January 1981 to January 1985 for all maturity forward rates. Since we are using a three-factor model, we take three consecutive forward rate maturities and find their $3 \times 3$ variance covariance matrix. We carry out a principal component analysis on this $3 \times 3$ covariance matrix. Principal component analysis decomposes the matrix into its eigenvalues and eigenvectors. The eigenvector corresponding to the largest eigenvalue gives the first principal component. When we multiply the square root of the largest eigenvalue by each of the three values in the eigenvector of the first principal component, we obtain the volatility estimates of three different maturity forward rates. We carry out this analysis for all different maturity forward rates. We use SAS to perform the principal component analysis.

For example, to estimate the volatilities of one-, two- and three-month forward rates, we find the changes in the monthly forward rates from January 1981 to December 1984. This gave us a time series of data for each of these three different maturity forward rates. Then we obtain the $3 \times 3$ covariance matrix of these forward rate changes. We run a principal component analysis on this covariance matrix to decompose it to its eigenvalues and eigenvectors. The largest eigenvalue is 4.42307 . The square root of this is 2.1031 . The eigenvector corresponding to the largest eigenvalue is $(0.6602930 .6602930 .357808)$. Therefore, the volatilities of the one-month and two month forward rates are $0.660293 * 2.1031=1.3887$. The SAS output of this example is attached at the end of this technical appendix. To allow continuity, the next PCA is carried out for three-, four- and five-month forward rates, and so forth until all different maturity forward rate volatilities are estimated. The estimated forward rate volatilities are given in Table TA1.

The McCulloch and Kwon (1993) data do not have forward rates of all months. We interpolated missing volatilities and smoothed these volatilities by the McCulloch (1975) cubic spline method. Bliss (1997) finds that the cubic spline method performs better than most of the other existing techniques to smooth and interpolate data. We used Mathematica for this cubic spline interpolation. The resulting forward rate volatilities of different maturities on January 1985 are given in Table TA2.

We fit these forward rate volatilities to four volatility functions: The constant volatility function of Ho an Lee (1986), the constant decay volatility considered in Au and Thurston (1995), the exponential decay volatility function of Vasicek (1977), and the humped volatility 
function of Mercurio and Moraleda (2000). The first two functions require one parameter, the exponential decay volatility requires two parameters, and the humped volatility requires three parameters. The estimation of these parameters is shown below for each volatility function of the forward rate volatilities on January 1985.

Constant Volatility $(\sigma(t, T)=\sigma)$

$\sigma(t, T)$ are the volatilities at time $t$ (January 1985) for different maturity forward rates. So, we have a cross-section of forward rate volatilities. We regress these volatilities on a constant ${ }^{2}$.

$$
\sigma(t, T)=\hat{\sigma}+\varepsilon
$$

We obtain an estimate of the constant volatility, $\hat{\sigma}$, of 0.03134 . This is the volatility of each different maturity forward rate on January 1985. The standard error is 9.81E-04, and the tstatistic is 31.95 .

Constant Decay Volatility $(\sigma(t, T)=\sigma /(1+T-t))$

The function $\sigma(t, T)$ represent the volatilities at time $\mathrm{t}$ (January 1985) for different maturity forward rates. If we multiply each of these volatilities by one plus time to maturity, $(1+T-t)$, we will have $\sigma(t, T)(1+T-t)=\sigma$ to satisfy the constant decay volatility function. If we regress these data on a constant, we obtain the parameter $\hat{\sigma}$.

$$
\sigma(t, T)(1+T-t)=\hat{\sigma}+\varepsilon
$$

We get $\hat{\sigma}$ as 0.57059 . Standard error is $3.49 \mathrm{E}-02$ and the t-statistic is 16.35 .

When we divide this $\hat{\sigma}$ by $(1+\mathrm{T}-\mathrm{t})$ for each different maturity forward rate, we obtain the constant decay volatility estimates for each forward rate on January 1985.

Exponentially Decaying Volatility $\left(\sigma(t, T)=\sigma e^{-\lambda(T-t)}\right)$

The function $\sigma(t, T)$ represent the volatilities we have at time $t$ (January 1985) for different maturity forward rates. This volatility function has two parameters, $\sigma$ and $\lambda$, that have to be estimated. If we take the natural logarithm of these volatilities we will have $\ln (\sigma(t, T))=\ln (\sigma)-\lambda(T-t)$. So we run the regression below:

\footnotetext{
${ }^{2}$ In other words, we take the expected value of these volatilities to find the volatility parameter of constant volatility.
} 
$\ln (\sigma(t, T))=\hat{\alpha}+\hat{\beta}(T-t)+\varepsilon$

In this equation, $\hat{\alpha}$ will give $\ln (\hat{\sigma})$. Therefore, to find $\hat{\sigma}$, we take $\exp (\hat{\alpha})$. We get $\hat{\alpha}$ as -0.77827 . The standard error of $\hat{\alpha}$ is $3.35 \mathrm{E}-02$, and the t-statistics is -23.23 . Since $\hat{\sigma}=\exp (\hat{\alpha})$, $\hat{\sigma}$ is $0.01591(\exp (-0.77827))$. In the above regression, $\hat{\beta}$ will give $-\lambda$. So $\lambda$ will be $-\hat{\beta}$. We get $\hat{\beta}$ as 0.03842 . The standard error of $\beta$ is $2.00 \mathrm{E}-03$, and the t-statistic is 19.21 . Therefore $\lambda$ is $0.03842^{3}$. According to these results, the volatility estimates of different maturity forward rates $\sigma(t, T)$ are given by $0.01591 * \exp (0.03842 *(\mathrm{~T}-\mathrm{t}))$.

Humped Volatility $\left(\sigma(t, T)=\sigma(1+\gamma(T-t)) e^{-\lambda(T-t)}\right)$

The function $\sigma(t, T)$ represent the volatilities at time $\mathrm{t}$ (January 1985) for different maturity forward rates. This volatility function has three parameters, $\sigma, \lambda$, and $\gamma$, that must be estimated. Similar to exponential decay volatility, we first take the natural logarithm of both sides of the volatility function to linearize part of the equation. This gives us $\ln (\sigma(t, T))=\ln (\sigma)-\lambda(T-t)+\ln (1+\gamma(T-t))$. Using a non-linear regression method to estimate the parameters, we run regression below, for which we use the Marquardt method. $\ln (\sigma(t, T))=\dot{\alpha}+\hat{\beta}(T-t)+\ln (1+\hat{\gamma}(T-t))+\varepsilon$

In this equation, $\hat{\alpha}$ will give $\ln (\hat{\sigma})$. Therefore, to find $\hat{\sigma}$, we take $\exp (\hat{\alpha})$. We get $\hat{\alpha}$ as -0.76567 and the standard error of $\hat{\alpha}$ is $3.38 \mathrm{E}-02$. Since $\hat{\sigma}=\exp (\hat{\alpha}), \hat{\sigma}$ is $0.01611 \quad(\exp (-$ $0.76567))$. In the above regression, $\hat{\beta}$ will give $-\lambda$. So $\lambda$ will be $-\hat{\beta}$. We get $\hat{\beta}$ as 0.03778 and the standard error of $\beta$ is $2.02 \mathrm{E}-03$. Hence $\lambda$ is -0.03778 . The relation between $\ln (\sigma(t, T))$ and $\hat{\gamma}$ is non-linear. We find $\hat{\gamma}$ as 6.383E-06 and the standard error is less than E-12. According to these results, the volatility estimates of different maturity forward rates, $\sigma(t, T)$, is estimated as $0.01611 *(1+6.38 \mathrm{E}-06 *(\mathrm{~T}-\mathrm{t})) * \exp (0.03778 *(\mathrm{~T}-\mathrm{t}))$.

\footnotetext{
${ }^{3}$ Amin and Morton (1994) also find that the volatility reduction function, $\lambda$, of the exponential decay volatility function is a negative value.
} 
Table TA1

\section{Forward Rate Volatility Estimates for January 1985}

Three-factor principal component analysis is used to estimate forward rate volatilities from the McCulloch and Kwon (1993) data.

\begin{tabular}{|r|r|}
\hline Maturity & Volatility \\
\hline 0 & 1.3887 \\
\hline 1 & 1.3887 \\
\hline 2 & 0.9403 \\
\hline 3 & 1.0960 \\
\hline 4 & 1.0144 \\
\hline 5 & 1.0132 \\
\hline 6 & 1.0144 \\
\hline 7 & 1.0473 \\
\hline 8 & 1.0109 \\
\hline 9 & 0.9210 \\
\hline 10 & 0.8060 \\
\hline 11 & 0.7656 \\
\hline 12 & 0.7418 \\
\hline 13 & 0.7403 \\
\hline 14 & 0.7311 \\
\hline 15 & 0.7402 \\
\hline 16 & 0.7372 \\
\hline 17 & 0.7606 \\
\hline 18 & 0.7688 \\
\hline 21 & 0.7809 \\
\hline 24 & 0.6606 \\
\hline 30 & 0.6697 \\
\hline 36 & 0.6159 \\
\hline 48 & 0.6341 \\
\hline 60 & 0.5956 \\
\hline & \\
\hline 1 &
\end{tabular}

\begin{tabular}{|r|r|}
\hline Maturity & Volatility \\
\hline 72 & 0.5657 \\
\hline 84 & 0.4997 \\
\hline 96 & 0.4660 \\
\hline 108 & 0.4591 \\
\hline 120 & 0.5908 \\
\hline 132 & 0.6579 \\
\hline 144 & 0.6737 \\
\hline 156 & 0.6176 \\
\hline 168 & 0.6250 \\
\hline 180 & 0.6114 \\
\hline 192 & 0.6660 \\
\hline 204 & 0.6645 \\
\hline 216 & 0.7016 \\
\hline 228 & 0.5551 \\
\hline 240 & 0.7366 \\
\hline 252 & 0.9004 \\
\hline 264 & 1.2356 \\
\hline 276 & 1.4168 \\
\hline 288 & 1.5403 \\
\hline 300 & 1.2252 \\
\hline 312 & 1.4255 \\
\hline 324 & 1.2500 \\
\hline 336 & 2.9987 \\
\hline 348 & 2.3009 \\
\hline 360 & 4.6242 \\
\hline & \\
\hline 250 &
\end{tabular}




\section{Table TA2}

\section{Smoothed and Interpolated Forward Rate Volatility Estimates for January 1985}

The cubic spline method is used to smooth the forward rate volatility estimates and to interpolate the missing ones.

\begin{tabular}{|r|r|}
\hline Maturity & Volatility \\
\hline 0 & 1.3887 \\
\hline 1 & 1.3887 \\
\hline 2 & 0.9403 \\
\hline 3 & 1.0960 \\
\hline 4 & 1.0144 \\
\hline 5 & 1.0132 \\
\hline 6 & 1.0144 \\
\hline 7 & 1.0473 \\
\hline 8 & 1.0109 \\
\hline 9 & 0.9210 \\
\hline 10 & 0.8060 \\
\hline 11 & 0.7656 \\
\hline 12 & 0.7418 \\
\hline 13 & 0.7403 \\
\hline 14 & 0.7311 \\
\hline 15 & 0.7402 \\
\hline 16 & 0.7372 \\
\hline 17 & 0.7606 \\
\hline 18 & 0.7688 \\
\hline 19 & 0.7817 \\
\hline 20 & 0.7908 \\
\hline 21 & 0.7809 \\
\hline 22 & 0.7439 \\
\hline 23 & 0.6951 \\
\hline 24 & 0.6606 \\
\hline 25 & 0.6543 \\
\hline 26 & 0.6546 \\
\hline 27 & 0.6589 \\
\hline 28 & 0.6647 \\
\hline 29 & 0.6689 \\
\hline 30 & 0.6697 \\
\hline & \\
\hline
\end{tabular}

\begin{tabular}{|r|r|}
\hline Maturity & Volatility \\
\hline 31 & 0.6647 \\
\hline 32 & 0.6560 \\
\hline 33 & 0.6443 \\
\hline 34 & 0.6323 \\
\hline 35 & 0.6226 \\
\hline 36 & 0.6159 \\
\hline 37 & 0.6146 \\
\hline 38 & 0.6145 \\
\hline 39 & 0.6154 \\
\hline 39 & 0.6171 \\
\hline 40 & 0.6193 \\
\hline 42 & 0.6220 \\
\hline 43 & 0.6248 \\
\hline 44 & 0.6275 \\
\hline 45 & 0.6301 \\
\hline 46 & 0.6321 \\
\hline 47 & 0.6335 \\
\hline 48 & 0.6341 \\
\hline 49 & 0.6336 \\
\hline 50 & 0.6322 \\
\hline 51 & 0.6300 \\
\hline 52 & 0.6271 \\
\hline 53 & 0.6236 \\
\hline 54 & 0.6198 \\
\hline 55 & 0.6157 \\
\hline 56 & 0.6114 \\
\hline 57 & 0.6071 \\
\hline 58 & 0.6030 \\
\hline 59 & 0.5991 \\
\hline 60 & 0.5956 \\
\hline 61 & 0.5925 \\
\hline & \\
\hline
\end{tabular}

\begin{tabular}{|r|r|}
\hline Maturity & Volatility \\
\hline 62 & 0.5899 \\
\hline 63 & 0.5877 \\
\hline 64 & 0.5856 \\
\hline 65 & 0.5837 \\
\hline 66 & 0.5818 \\
\hline 67 & 0.5798 \\
\hline 68 & 0.5777 \\
\hline 69 & 0.5754 \\
\hline 70 & 0.5726 \\
\hline 71 & 0.5694 \\
\hline 72 & 0.5657 \\
\hline 73 & 0.5613 \\
\hline 74 & 0.5564 \\
\hline 75 & 0.5510 \\
\hline 76 & 0.5453 \\
\hline 77 & 0.5394 \\
\hline 78 & 0.5333 \\
\hline 79 & 0.5272 \\
\hline 80 & 0.5211 \\
\hline 81 & 0.5153 \\
\hline 82 & 0.5097 \\
\hline 83 & 0.5044 \\
\hline 84 & 0.4997 \\
\hline 85 & 0.4954 \\
\hline 86 & 0.4917 \\
\hline 87 & 0.4885 \\
\hline 88 & 0.4855 \\
\hline 89 & 0.4829 \\
\hline 90 & 0.4805 \\
\hline 91 & 0.4782 \\
\hline 92 & 0.4759 \\
\hline & \\
\hline
\end{tabular}

\begin{tabular}{|r|r|}
\hline Maturity & Volatility \\
\hline 93 & 0.4737 \\
\hline 94 & 0.4713 \\
\hline 95 & 0.4688 \\
\hline 96 & 0.4660 \\
\hline 97 & 0.4630 \\
\hline 98 & 0.4598 \\
\hline 99 & 0.4566 \\
\hline 100 & 0.4537 \\
\hline 101 & 0.4512 \\
\hline 102 & 0.4492 \\
\hline 103 & 0.4480 \\
\hline 104 & 0.4476 \\
\hline 105 & 0.4484 \\
\hline 106 & 0.4505 \\
\hline 107 & 0.4540 \\
\hline 108 & 0.4591 \\
\hline 109 & 0.4660 \\
\hline 110 & 0.4744 \\
\hline 111 & 0.4842 \\
\hline 112 & 0.4950 \\
\hline 113 & 0.5066 \\
\hline 114 & 0.5189 \\
\hline 115 & 0.5314 \\
\hline 116 & 0.5441 \\
\hline 117 & 0.5566 \\
\hline 118 & 0.5687 \\
\hline 119 & 0.5802 \\
\hline 120 & 0.5908 \\
\hline 121 & 0.6004 \\
\hline 122 & 0.6089 \\
\hline 123 & 0.6164 \\
\hline & \\
\hline
\end{tabular}


Table TA2 (Cont'd)

Smoothed and Interpolated Forward Rate Volatility Estimates for January 1985

\begin{tabular}{|r|r|}
\hline Maturity & Volatility \\
\hline 124 & 0.6231 \\
\hline 125 & 0.6291 \\
\hline 126 & 0.6344 \\
\hline 127 & 0.6392 \\
\hline 128 & 0.6435 \\
\hline 129 & 0.6474 \\
\hline 130 & 0.6510 \\
\hline 131 & 0.6545 \\
\hline 132 & 0.6579 \\
\hline 133 & 0.6612 \\
\hline 134 & 0.6645 \\
\hline 135 & 0.6676 \\
\hline 136 & 0.6704 \\
\hline 137 & 0.6729 \\
\hline 138 & 0.6750 \\
\hline 139 & 0.6765 \\
\hline 140 & 0.6775 \\
\hline 141 & 0.6778 \\
\hline 142 & 0.6773 \\
\hline 143 & 0.6759 \\
\hline 144 & 0.6737 \\
\hline 145 & 0.6704 \\
\hline 146 & 0.6662 \\
\hline 147 & 0.6614 \\
\hline 148 & 0.6561 \\
\hline 149 & 0.6505 \\
\hline 150 & 0.6448 \\
\hline 151 & 0.6391 \\
\hline 152 & 0.6336 \\
\hline 153 & 0.6286 \\
\hline 154 & 0.6241 \\
\hline & \\
\hline
\end{tabular}

\begin{tabular}{|r|r|}
\hline Maturity & Volatility \\
\hline 155 & 0.6203 \\
\hline 156 & 0.6176 \\
\hline 157 & 0.6158 \\
\hline 158 & 0.6151 \\
\hline 159 & 0.6151 \\
\hline 160 & 0.6158 \\
\hline 161 & 0.6170 \\
\hline 162 & 0.6184 \\
\hline 163 & 0.6201 \\
\hline 164 & 0.6217 \\
\hline 165 & 0.6232 \\
\hline 166 & 0.6243 \\
\hline 167 & 0.6250 \\
\hline 168 & 0.6250 \\
\hline 169 & 0.6242 \\
\hline 170 & 0.6228 \\
\hline 171 & 0.6210 \\
\hline 172 & 0.6188 \\
\hline 173 & 0.6166 \\
\hline 174 & 0.6143 \\
\hline 175 & 0.6123 \\
\hline 176 & 0.6107 \\
\hline 177 & 0.6096 \\
\hline 178 & 0.6093 \\
\hline 179 & 0.6098 \\
\hline 180 & 0.6114 \\
\hline 181 & 0.6142 \\
\hline 182 & 0.6180 \\
\hline 183 & 0.6226 \\
\hline 184 & 0.6278 \\
\hline 185 & 0.6335 \\
\hline & \\
\hline
\end{tabular}

\begin{tabular}{|r|r|}
\hline Maturity & Volatility \\
\hline 186 & 0.6393 \\
\hline 187 & 0.6450 \\
\hline 188 & 0.6505 \\
\hline 189 & 0.6556 \\
\hline 190 & 0.6600 \\
\hline 191 & 0.6636 \\
\hline 192 & 0.6660 \\
\hline 193 & 0.6673 \\
\hline 194 & 0.6675 \\
\hline 195 & 0.6668 \\
\hline 196 & 0.6656 \\
\hline 197 & 0.6641 \\
\hline 198 & 0.6625 \\
\hline 199 & 0.6610 \\
\hline 200 & 0.6600 \\
\hline 201 & 0.6596 \\
\hline 202 & 0.6600 \\
\hline 203 & 0.6616 \\
\hline 204 & 0.6645 \\
\hline 205 & 0.6690 \\
\hline 206 & 0.6746 \\
\hline 207 & 0.6810 \\
\hline 208 & 0.6877 \\
\hline 209 & 0.6942 \\
\hline 210 & 0.7003 \\
\hline 211 & 0.7054 \\
\hline 212 & 0.7091 \\
\hline 213 & 0.7110 \\
\hline 214 & 0.7107 \\
\hline 215 & 0.7077 \\
\hline 216 & 0.7016 \\
\hline & \\
\hline
\end{tabular}

\begin{tabular}{|r|r|}
\hline Maturity & Volatility \\
\hline 217 & 0.6921 \\
\hline 218 & 0.6799 \\
\hline 219 & 0.6655 \\
\hline 220 & 0.6497 \\
\hline 221 & 0.6331 \\
\hline 222 & 0.6165 \\
\hline 223 & 0.6006 \\
\hline 224 & 0.5860 \\
\hline 225 & 0.5735 \\
\hline 226 & 0.5637 \\
\hline 227 & 0.5573 \\
\hline 228 & 0.5551 \\
\hline 229 & 0.5575 \\
\hline 230 & 0.5642 \\
\hline 231 & 0.5745 \\
\hline 232 & 0.5880 \\
\hline 233 & 0.6039 \\
\hline 234 & 0.6218 \\
\hline 235 & 0.6409 \\
\hline 236 & 0.6609 \\
\hline 237 & 0.6810 \\
\hline 238 & 0.7007 \\
\hline 239 & 0.7195 \\
\hline 240 & 0.7366 \\
\hline 241 & 0.7518 \\
\hline 242 & 0.7652 \\
\hline 243 & 0.7774 \\
\hline 244 & 0.7887 \\
\hline 245 & 0.7996 \\
\hline 246 & 0.8105 \\
\hline 247 & 0.8218 \\
\hline & \\
\hline
\end{tabular}


Table TA2 (Cont'd)

Smoothed and Interpolated Forward Rate Volatility Estimates for January 1985

\begin{tabular}{|r|r|}
\hline Maturity & Volatility \\
\hline 248 & 0.8340 \\
\hline 249 & 0.8476 \\
\hline 250 & 0.8628 \\
\hline 251 & 0.8803 \\
\hline 252 & 0.9004 \\
\hline 253 & 0.9233 \\
\hline 254 & 0.9489 \\
\hline 255 & 0.9764 \\
\hline 256 & 1.0056 \\
\hline 257 & 1.0358 \\
\hline 258 & 1.0666 \\
\hline 259 & 1.0974 \\
\hline 260 & 1.1279 \\
\hline 261 & 1.1574 \\
\hline 262 & 1.1855 \\
\hline 263 & 1.2118 \\
\hline 264 & 1.2356 \\
\hline 265 & 1.2567 \\
\hline 266 & 1.2753 \\
\hline 267 & 1.2919 \\
\hline 268 & 1.3068 \\
\hline 269 & 1.3205 \\
\hline 270 & 1.3334 \\
\hline 271 & 1.3459 \\
\hline 272 & 1.3584 \\
\hline 273 & 1.3713 \\
\hline 274 & 1.3851 \\
\hline 275 & 1.4001 \\
\hline 276 & 1.4168 \\
\hline 277 & 1.4355 \\
\hline 278 & 1.4553 \\
\hline & \\
\hline
\end{tabular}

\begin{tabular}{|r|r|}
\hline Maturity & Volatility \\
\hline 279 & 1.4756 \\
\hline 280 & 1.4956 \\
\hline 281 & 1.5142 \\
\hline 282 & 1.5308 \\
\hline 283 & 1.5445 \\
\hline 284 & 1.5545 \\
\hline 285 & 1.5599 \\
\hline 286 & 1.5599 \\
\hline 287 & 1.5536 \\
\hline 288 & 1.5403 \\
\hline 289 & 1.5195 \\
\hline 290 & 1.4924 \\
\hline 291 & 1.4606 \\
\hline 292 & 1.4257 \\
\hline 293 & 1.3892 \\
\hline 294 & 1.3528 \\
\hline 295 & 1.3181 \\
\hline 296 & 1.2866 \\
\hline 297 & 1.2600 \\
\hline 298 & 1.2398 \\
\hline 299 & 1.2277 \\
\hline 300 & 1.2252 \\
\hline 301 & 1.2332 \\
\hline 302 & 1.2503 \\
\hline 303 & 1.2741 \\
\hline 304 & 1.3025 \\
\hline 305 & 1.3332 \\
\hline 306 & 1.3639 \\
\hline 307 & 1.3926 \\
\hline 308 & 1.4168 \\
\hline 309 & 1.4345 \\
\hline & \\
\hline
\end{tabular}

\begin{tabular}{|r|r|}
\hline Maturity & Volatility \\
\hline 310 & 1.4433 \\
\hline 311 & 1.4411 \\
\hline 312 & 1.4255 \\
\hline 313 & 1.3956 \\
\hline 314 & 1.3543 \\
\hline 315 & 1.3060 \\
\hline 316 & 1.2549 \\
\hline 317 & 1.2051 \\
\hline 318 & 1.1609 \\
\hline 319 & 1.1266 \\
\hline 320 & 1.1063 \\
\hline 321 & 1.1043 \\
\hline 322 & 1.1247 \\
\hline 323 & 1.1719 \\
\hline 324 & 1.2500 \\
\hline 325 & 1.3615 \\
\hline 326 & 1.5014 \\
\hline 327 & 1.6631 \\
\hline 328 & 1.8400 \\
\hline 329 & 2.0254 \\
\hline 330 & 2.2125 \\
\hline 331 & 2.3947 \\
\hline 332 & 2.5653 \\
\hline 333 & 2.7177 \\
\hline 334 & 2.8452 \\
\hline 335 & 2.9411 \\
\hline 336 & 2.9987 \\
\hline 337 & 3.0137 \\
\hline 338 & 2.9910 \\
\hline 339 & 2.9380 \\
\hline 340 & 2.8620 \\
\hline & \\
\hline
\end{tabular}

\begin{tabular}{|r|r|}
\hline Maturity & Volatility \\
\hline 341 & 2.7702 \\
\hline 342 & 2.6700 \\
\hline 343 & 2.5687 \\
\hline 344 & 2.4735 \\
\hline 345 & 2.3919 \\
\hline 346 & 2.3310 \\
\hline 347 & 2.2983 \\
\hline 348 & 2.3009 \\
\hline
\end{tabular}




\section{Figure TA1}

\section{Principal Component Analysis by SAS}

This figure shows the SAS output of an example of principal component analysis carried out to find the forward rate volatilities.

The SAS System

Principal Component Analysis

48 Observations

3 Variables

\section{Simple Statistics}

$\mathrm{CF} 1$

Mean
StD

$-0.073306122$

1.401106143

$-0.073306122$

1.401106143

$-0.132020408$

1.019275713

Covariance Matrix

CF1

1.963098425

0.916922389

1.963098425
1.963098425

1.963098425

0.916922389

Total Variance $=4.9651198291$

Eigenvalues of the Covariance Matrix
Eigenvalue

4.42307

0.54205

0.00000
Difference

$$
3.88102
$$$$
0.54205
$$$$
\text { . }
$$

Eigenvectors

\begin{tabular}{ccrr}
\multicolumn{4}{c}{ Eigenvectors } \\
& PRIN1 & PRIN2 & PRIN3 \\
CF1 & 0.660293 & -.253008 & 0.707107 \\
CF2 & 0.660293 & -.253008 & -.707107
\end{tabular}

$\mathrm{CF} 3$

0.916922389

0.916922389

1.038922979 
$\begin{array}{lll}1 & 1.38867 & 1.96310\end{array}$

1.38867

1.9631 


\section{VITA}

Şenay Ağca was born in Ankara, Turkey. She earned a Bachelor's degree in Industrial Engineering, and an MBA degree from Middle East Technical University (METU), Ankara, Turkey. She joined the Ph.D. program of Virginia Tech in 1997, and earned her Ph.D. degree in March 2002. She will join the Finance Department of The George Washington University as an assistant professor in August 2002. 